\title{
Hyphenating the Curtius Rearrangement with Morita-Baylis-Hillman Adducts: Synthesis of Biologically Active Acyloins and Vicinal Aminoalcohols
}

\author{
Giovanni W. Amarante, Mayra Cavallaro and Fernando Coelho* \\ Laboratório de Síntese de Produtos Naturais e Fármacos, Instituto de Química, \\ Universidade de Campinas, 13083-970 Campinas-SP, Brazil
}

\begin{abstract}
Um rearranjo de Curtius, utilizando adutos de Morita-Baylis-Hillman como substrato, foi realizado em uma sequência que permitiu a síntese de várias hidroxi-cetonas (aciloínas) com uma grande diversidade estrutural e com bons rendimentos globais. Por sua vez, essas aciloínas foram transformadas em 1,2-amino-alcoóis de configuração relativa anti, através de uma etapa de aminação redutiva altamente diastereosseletiva. A utilidade sintética dessas abordagens foi demonstrada através das sínteses totais da $( \pm)$-bupropiona, fármaco utilizado no tratamento na síndrome de abstinência de fumantes e da ( \pm )-espisulosina, um potente agente anti-tumoral isolado inicialmente de uma fonte marinha.
\end{abstract}

Using Morita-Baylis-Hillman adducts as substrates, the Curtius rearrangement was performed in a sequence that allowed the synthesis of several hydroxy-ketones (acyloins) with great structural diversity and in good overall yields. These acyloins in turn were easily transformed into 1,2-anti aminoalcohols through a highly diastereoselective reductive amination step. The synthetic utility of these approaches was exemplified by performing the syntheses of ( \pm )-bupropion, a drug used to treat the abstinence syndrome of smoker and ( \pm )-spisulosine, a potent anti-tumoral compound originally isolated from a marine source.

Keywords: Curtius rearrangement, Morita-Baylis-Hillman, drugs, aminoalcohols, acyloins

\section{Introduction}

The formation of new $\mathrm{C}-\mathrm{N}$ bonds by incorporation of a nitrogen atom into a molecule is a fundamental transformation in organic chemistry, since it allows accessing many valuable compounds. ${ }^{1}$ Basically, this can be achieved by nucleophilic substitution reactions or by electrophilic amination reactions. ${ }^{2}$

A C-N bond can also be built through rearrangement reactions. For instance, Lossen, ${ }^{3}$ Beckman, ${ }^{4}$ Schmidt $^{5}$ and Curtius $^{6}$ rearrangements are processes which allow the efficient formation of a new $\mathrm{C}-\mathrm{N}$ bond from carbonyl containing derivative compounds.

The Curtius rearrangement is a thermal decomposition of a carbonyl azide leading to an isocyanate. This stereospecific rearrangement provides carbamates or amines in good overall yields and selectivities. Unfortunately, this rearrangement has the drawback that low molar mass acyl azides present an explosion hazard. ${ }^{7}$ This safety issue

*e-mail: coelho@iqm.unicamp.br has limited the industrial use of this transformation until recently, when Am Ende et al. ${ }^{8}$ reported a new experimental protocol, which enabled the use of this reaction under safer conditions even when conducted on a large scale. This and other protocols have revived interest in this rearrangement, particularly for industrial purposes. ${ }^{9}$

Regarding the potential of this rearrangement, our group has recently reported the preparation of some carbamates from Morita-Baylis-Hillman (MBH) adducts. ${ }^{10}$ Curtius rearrangement would be the best way to achieve our target, since the carboxyl group in this structure is needed to perform this rearrangement. Thus, when an acid derived from a $\mathrm{MBH}$ adduct was treated with sodium azide, heated at reflux and treated subsequently with $t$-butanol, the corresponding ene-carbamate was formed in good overall yield. However, in some examples it was observed the occurrence of a byproduct ( $c a .12-15 \%)$ which was characterized as being an acyloin (Scheme 1).

The acyloin formation was easily explained by the presence of water (tiny amount) in the $t-\mathrm{BuOH}$ used to transform the intermediate ene-isocyanate into the 


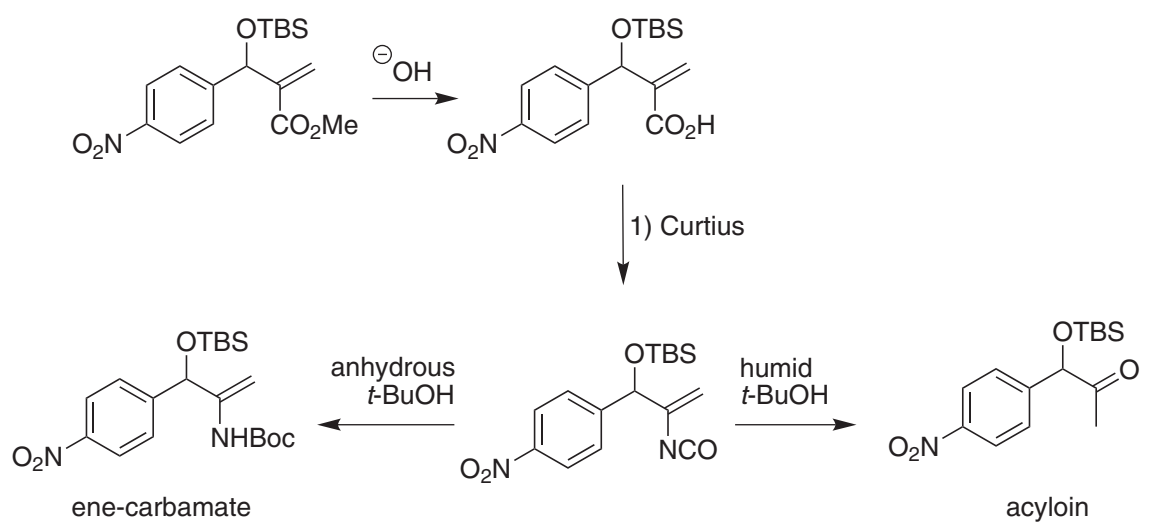

Scheme 1. Curtius rearrangement using Morita-Baylis-Hillman adduct as substrate.

corresponding ene-carbamate. This undesirable side reaction caught our attention, since $\alpha$-hydroxyketones (acyloins) are structural motifs present in several biologically active compounds, in which the activity is closely associated with the presence of this structural pattern. Due to the synthetic and biological relevance of acyloins, several synthetic approaches have already been developed in order to prepare them. Conventionally, $\alpha$-hydroxy ketones are prepared by the acyloin condensation reaction, ${ }^{11}$ oxidation of enolates or double bonds, ${ }^{12}$ or reduction of $\alpha$-diketones or esters. ${ }^{13}$ Recently, a method based on ketohydroxylation of alkenes was developed to give acyloins. ${ }^{14}$ Alternatively, radical oxidation of a 1,3-dicarbonyl compound with cerium salts could also be used for the preparation of acyloins. ${ }^{15}$ Most recently, a skeletal rearrangement of symmetrically $\alpha, \alpha$-disubstituted $\alpha$-amino aldehyde has been reported as a new strategy for preparing acyloins. ${ }^{16}$

Beyond its biological importance, $\alpha$-hydroxyketones are also an interesting synthetic platform that allows further chemical transformations. For instance, a $\alpha$-hydroxyketone can be easily transformed into a $\alpha$-aminoketone. This structural pattern is present in some commercial drugs, such as bupropion [( \pm )- $\alpha$-t-butylamino-3-chloropropiophenone] (1, Figure 1), a potent synthetic inhibitor of dopamine reuptake with subtle noradrenergic reuptake. ${ }^{17}$ Bupropion is an atypical antidepressant, which has been licensed by FDA to treat the abstinence syndrome of smokers. ${ }^{18}$

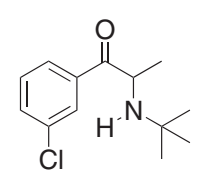

( \pm )-bupropion (1)

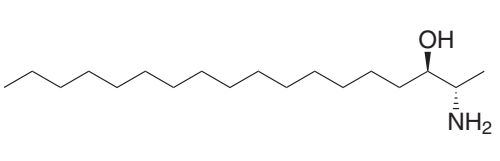

( \pm )-spisulosine (2)
Figure 1. Molecular structures of bupropion and spisulosine.

Vicinal aminoalcohols (or 1,2-aminoalcohols) are structural units that currently occur in several biologically active compounds. They are also part of several chiral catalysts and new materials.

The biological and synthetic importances of this structural motif have stimulated the development of several methods to synthesize it, both in racemic and enantiomerically pure forms. ${ }^{19}$ Classically, syn-1,2-aminoalcohols can be prepared in their racemic or asymmetric versions using the aminohydroxylation strategy developed by Sharpless et al..$^{20}$ Another efficient way to prepare vicinal aminoalcohols is by the opening of an epoxide ring with a suitable nitrogen source, such as amines or azide ions, to provide aminoalcohols or azidoalcohols, respectively. ${ }^{21,22}$

By using simple chemical transformations, $\alpha$-hydroxyketones (acyloins) can also be used as substrates for the stereoselective preparation of 1,2-aminoalcohols. ${ }^{23}$

Spisulosine (2, Figure 1) is a sphingoid-type base which presents a long unsaturated alkyl chain $\left(\mathrm{C}_{15}\right)$ and a 1,2-aminoalcohol motif in an anti relationship. ${ }^{24}$ This compound was isolated from extracts obtained from the clam Spisula polynyma and exhibits a promising activity against prostate cancer. ${ }^{25}$

In a research program directed towards the total synthesis of drugs from Morita-Baylis-Hillman adducts, ${ }^{26}$ we describe herein the synthesis of $\alpha$-hydroxyketones and their diastereoselective transformations into anti-1,2-aminoalcohols. To exemplify the synthetic utility of this approach, we also describe the syntheses of the drugs (+/-)-bupropion and (+/-)-spisulosine.

\section{Results and Discussion}

The present work began by preparing the $\mathrm{MBH}$ adducts, according to a procedure previously developed in our laboratory. ${ }^{27}$ The strategy provides the corresponding adducts in good to high yields. The results are summarized in Table 1.

Initially, the Curtius rearrangement without protection of the secondary hydroxyl group was of interesting in this 
Table 1. Morita-Baylis-Hillman adducts

\begin{tabular}{lcc}
\hline Entry & Aldehyde & MBH adduct, yield / \% \\
\hline 1 & 2-nitrobenzaldehyde & $\mathbf{3}, 92$ \\
2 & 3-chlorobenzaldehyde & $\mathbf{4}, 89$ \\
3 & benzaldehyde & $\mathbf{5 , 7 5}$ \\
4 & 4-methoxybenzaldehyde & $\mathbf{6 , 7 7}$ \\
5 & 3,4,5-trimethoxybenzaldehyde & $\mathbf{7}, 60$ \\
6 & thiophenecarboxaldehyde & $\mathbf{8 , 9 0}$ \\
7 & 2-fluorobenzaldehyde & $\mathbf{9 , 9 5}$ \\
8 & propanaldehyde & $\mathbf{1 0}, 80$ \\
9 & hexanaldehyde & $\mathbf{1 1}, 76$ \\
10 & hexadecanaldehyde & $\mathbf{1 2}, 60$ \\
\hline
\end{tabular}

${ }^{a}$ Yields refer to isolated and purified products; ${ }^{b} \mathrm{MBH}$ reaction was performed with excess of methyl acrylate in the presence of [bmim] $\mathrm{PF}_{6}$ at $0{ }^{\circ} \mathrm{C}$; c prepared by oxidation of commercial hexadecanol with PCC in refluxing dichloromethane in $95 \%$ yield

work. So, adduct 3 was hydrolysed in the presence of $\mathrm{LiOH}$ in an acetonitrile: $\mathrm{H}_{2} \mathrm{O}(1: 1)$ mixture to give a hydroxyacid in almost quantitative yield (>98\%). When this acid was treated with ethyl chloroformate, extensive degradation of our starting material was observed by TLC analysis.

In an attempt to circumvent this issue, the secondary hydroxyl group of the MBH adducts was protected, before ester hydrolysis. Thus, adducts 3-12 were treated with TBSCl or TBSOTf in DMF or dichloromethane, in the presence of imidazole or triethylamine, respectively. The silylated adducts (13-22) were obtained in good to excellent yields. Soon after, ester hydrolysis was carried out with $\mathrm{LiOH}$, in an acetonitrile: $\mathrm{H}_{2} \mathrm{O}$ mixture at $60{ }^{\circ} \mathrm{C}$ to produce acids 23-32 in excellent yields. The results of these steps are summarized in Table 2.

The Curtius rearrangement was initiated using diphenylphosphoryl azide (DPPA). ${ }^{28}$ This reagent is supposed to react with a carboxylic acid to provide an isocyanate in a single step. A solution of silyl-acid $\mathbf{2 3}$ in toluene was therefore treated with DPPA at reflux for $20 \mathrm{~h}$. Unfortunately, it was unable to isolate any isocyanate. Instead, a complete destruction of the silylated acid was observed.

Because of this result, it was decided to perform the Curtius rearrangement by using a classical experimental protocol. Thus, an acetone solution of silylated acids was treated with ethyl chloroformate in the presence of triethylamine at $0{ }^{\circ} \mathrm{C}$ for $5 \mathrm{~min}$. After that, sodium azide was added to the reaction mixture and this mixture was vigorously stirred for $2 \mathrm{~h}$. The crude products were refluxed in dry toluene for $2 \mathrm{~h}$ to give the corresponding isocyanates, which finally were refluxed in water to furnish a set of acyloins in good overall yield (ranging from 35 to $50 \%$ for 3 steps). The results are summarized in Table 3.

Acyloins were obtained in good overall yield in three steps from Morita-Baylis-Hillman adducts. The strategy is simple and requires no special conditions such as low temperature, dry solvents or special catalysts. Moreover, it uses reagents which are routinely found in organic synthesis laboratories.

Table 2. Silylation and hydrolysis of Morita-Baylis-Hillman adducts

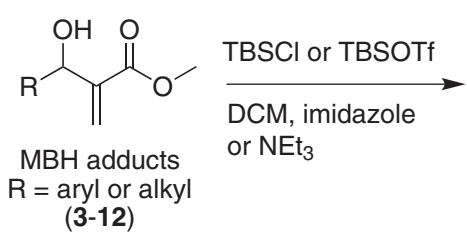

(3-12)<smiles>[R]C(O)C(=O)C(=O)OC</smiles>

(13-22)

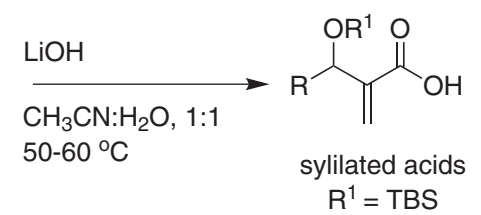

(23-32)

\begin{tabular}{lccc}
\hline Entry & MBH adducts & Silylated adducts, yield $/ \%^{\mathrm{a}}$ & \multicolumn{2}{c}{${\text { Silylated acids, yield } / \%^{\mathrm{a}}}^{\mathrm{23}, 97}$} \\
\hline 1 & $\mathbf{3}$ & $\mathbf{1 3}, 97$ & $\mathbf{2 4 ,}, 99$ \\
2 & $\mathbf{4}$ & $\mathbf{1 4}, 99$ & $\mathbf{2 5}, 99$ \\
3 & $\mathbf{5}$ & $\mathbf{1 5}, 99$ & $\mathbf{2 6}, 98$ \\
4 & $\mathbf{6}$ & $\mathbf{1 6}, 99$ & $\mathbf{2 7}, 99$ \\
5 & $\mathbf{7}$ & $\mathbf{1 7}, 99$ & $\mathbf{2 8}, 99$ \\
6 & $\mathbf{8}$ & $\mathbf{1 8}, 90$ & $\mathbf{2 9}, 99$ \\
7 & $\mathbf{9}$ & $\mathbf{1 9}, 85$ & $\mathbf{3 0}, 99$ \\
9 & $\mathbf{2 0}, 85$ & $\mathbf{2 1}, 99$ & $\mathbf{3 1}, 98$ \\
10 & $\mathbf{1 1}$ & $\mathbf{2 2 ,}, 70$ & $\mathbf{3 2}, 99^{\mathrm{b}}$ \\
\hline
\end{tabular}

${ }^{a}$ Yields refer to isolated and purified products; ${ }^{b}$ methanol used as solvent and $\mathrm{NaOH}$ used as base instead of $\mathrm{LiOH}$. 
Table 3. Acyloins from Morita-Baylis-Hillman adducts<smiles>[R]C(OC)C(=O)C(=O)O</smiles>

i) $\mathrm{ClCO}_{2} \mathrm{Et}$, acetone, $5^{\circ} \mathrm{C}, 5 \mathrm{~min}$

ii) $\mathrm{NaN}_{3}, \mathrm{r} ., 2 \mathrm{~h}$

iii) toluene, reflux, $2 \mathrm{~h}$

iv) $\mathrm{H}_{2} \mathrm{O}$, reflux, $2 \mathrm{~h}$

$\mathrm{R}^{1}=\mathrm{TBS}$

23-32

\begin{tabular}{lccc}
\hline Entry & $\mathrm{R}$ & Acyloin & Yield / \% a,b $^{\mathrm{b}}$ \\
\hline 1 & 2-nitrophenyl & $\mathbf{3 3}$ & 46 \\
2 & 3-chlorophenyl & $\mathbf{3 4}$ & 45 \\
3 & phenyl & $\mathbf{3 5}$ & 50 \\
4 & 4-methoxyphenyl & $\mathbf{3 6}$ & 48 \\
5 & 3,4,5-trimethoxyphenyl & $\mathbf{3 7}$ & 42 \\
6 & thienyl & $\mathbf{3 8}$ & 44 \\
7 & 2-fluorophenyl & $\mathbf{3 9}$ & 41 \\
8 & ethyl & $\mathbf{4 0}$ & 46 \\
9 & hexyl & $\mathbf{4 1}$ & 42 \\
10 & hexadecanyl & $\mathbf{4 2}$ & 40 \\
\hline
\end{tabular}

${ }^{a}$ Yields refer to isolated and purified products; ${ }^{b}$ overall yield (3 steps from silyl esters).

The bifunctional identity (one eletrophilic group and another nucleophilic) makes acyloins an important building block in organic synthesis. They can be converted into several different functional groups, such as alcohols, diols, epoxides, amines, hydroxylamines and haloketones. This synthetic versatility explains the frequent use of acyloins as building blocks for the synthesis of pharmaceutical compounds. ${ }^{29}$

A ketone carbonyl group can be promptly transformed into an amine by a reductive amination reaction. Applications of this reaction are widespread in the pharmaceutical, agrochemical and chemical industries, and in materials science and biotechnology. ${ }^{30-32}$

Recently, Cabral et $a l .{ }^{33}$ have developed a simple method to perform a reductive amination, based on in situ formation of an iminium, followed by reduction with $\mathrm{LiBH}_{4}$. Thus, this was viewed in the present work as an attractive opportunity to test this method to prepare vicinal aminoalcohols from our acyloins.

A methanol solution of $\mathbf{3 5}$ was treated with $\mathrm{LiBH}_{4}$ in the presence of an excess of benzylamine ( 3 equiv.) at $-72{ }^{\circ} \mathrm{C}$ to give aminoalcohol 46 in $83 \%$ yield and excellent 1,2-anti diastereoselectivity (97:3 d.r., anti:syn). This simple sequence has allowed the development of a highly diastereoselective synthesis of several different aminoalcohols in good yields. The results are summarized in Table 4.

For all cases examined, the vicinal aminoalcohols were obtained in good yields and with excellent diastereoselectivities. To confirm the 1,2-anti relative stereochemistry, the silyl protecting group of aminoalcohol $\mathbf{4 4}$ was removed by treatment with TBAF. The unprotected vicinal aminoalcohol $\mathbf{5 5}$ was reacted with triphosgene to

Table 4. Vicinal aminoalcohols from acyloins

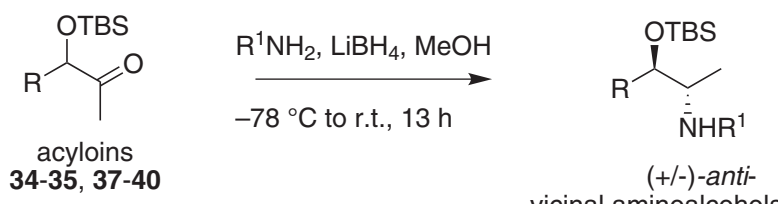

vicinal aminoalcohols (43-54)

\begin{tabular}{|c|c|c|c|c|}
\hline Entry & Acyloin $(\mathrm{R})$ & Amine $\left(\mathrm{R}^{1}\right)$ & Yield / \% ${ }^{\mathrm{a}}$ & d.r. ${ }^{b}$ \\
\hline 1 & 34, 3-chlorophenyl & phenethyl & 43,79 & $96: 4$ \\
\hline 2 & 34, 3-chlorophenyl & benzyl & $\mathbf{4 4 ,}, 77$ & $92: 8$ \\
\hline 3 & 35, phenyl & phenethyl & 45,85 & $>95: 5$ \\
\hline 4 & 35, phenyl & benzyl & $\mathbf{4 6}, 83$ & $97: 3$ \\
\hline 5 & 35, phenyl & allyl & 47,82 & $98: 2$ \\
\hline 6 & 35, phenyl & cyclohexyl & 48,80 & $93: 7$ \\
\hline 7 & 37, 3,4,5-trimethoxyphenyl & phenethyl & $\mathbf{4 9}, 81$ & $>95: 5$ \\
\hline 8 & 37, 3,4,5-trimethoxyphenyl & allyl & $\mathbf{5 0}, 80$ & $87: 13$ \\
\hline 9 & 38, thienyl & phenethyl & $\mathbf{5 1}, 82$ & $91: 9$ \\
\hline 10 & 39, 2-fluorophenyl & phenethyl & $\mathbf{5 2}, 78$ & $>95: 5$ \\
\hline 11 & 40, ethyl & phenethyl & $\mathbf{5 3}, 73$ & $>95: 5$ \\
\hline 12 & 40, ethyl & decyl & $\mathbf{5 4}, 71$ & $>95: 5$ \\
\hline
\end{tabular}

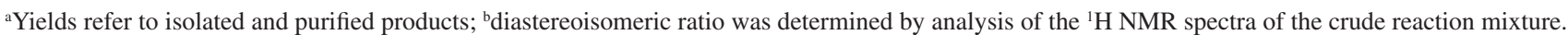
The carbinolic proton shows a doublet with a coupling constant of $5 \mathrm{~Hz}$ for the anti relationship, while the syn product shows a coupling constant of $8.2 \mathrm{~Hz}$. 


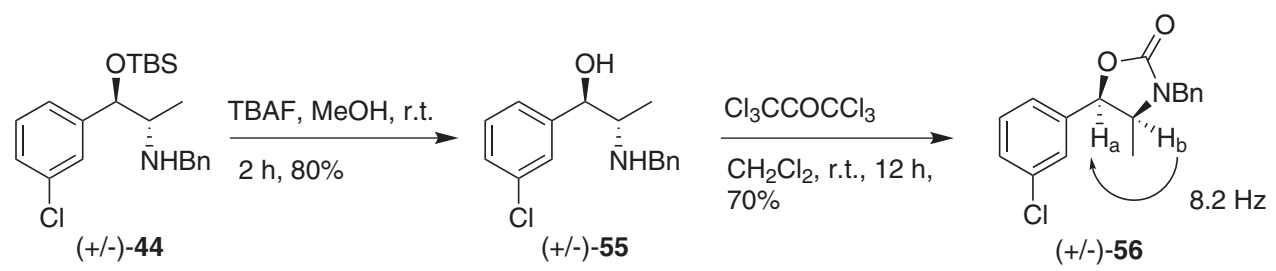

Scheme 2. Relative stereochemistry assignment of the 1,2-anti aminoalcohol derivatives.

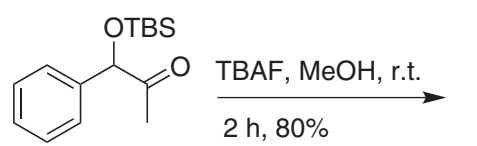

$(+/-)-35$<smiles>CC(=O)C(O)c1ccccc1</smiles>

$(+/-)-57$

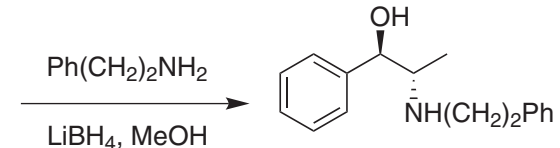

$(+/-)-58$

anti:syn, 87:13

Scheme 3. Silyl protecting group and diastereoselectivity.

afford oxazolidinone 56, in 70\% yield (Scheme 2). The coupling constant between hydrogens $\mathrm{H}_{\mathrm{a}}$ and $\mathrm{H}_{\mathrm{b}}$ was found to be $8.2 \mathrm{~Hz}$, which it is in agreement with typical coupling constants for a cis relationship in oxazolidinones formed from a 1,2-anti aminoalcohol (Scheme 2). ${ }^{34}$

The presence of a bulky protecting group on the secondary hydroxyl group might play an important role in the iminium reduction step. Most likely the hydride attack occurs at the opposite side from the protecting group. To experimentally validate this hypothesis, silylated acyloin 35 was treated with TBAF in methanol to provide acyloin $\mathbf{5 7}$ in $80 \%$ yield. A methanol solution of $\mathbf{5 7}$ at $-72{ }^{\circ} \mathrm{C}$ was treated with $\mathrm{LiBH}_{4}$ to afford aminoalcohol $\mathbf{5 8}$ in $70 \%$ yield and moderate diastereoselectivity (87:13 d.r.; anti:syn) (Scheme 3).

This result shows the effect of the silyl protecting group on the diastereoselectivity of the reduction step. Besides, the lithium atom could be complexed both with the nitrogen and the oxygen lone pairs (Figure 2). This arrangement allows the hydride to approach only from the

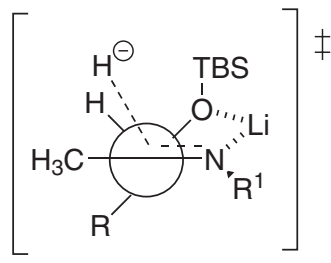

Figure 2. Rationalizing the diastereoselectivity. less hindered side. The oxygen atom attached to the silicon of the TBS group has a low basicity, however it is still able to establish intramolecular hydrogen bonds in order to control stereoselectivities of reactions. ${ }^{35}$

The Curtius rearrangement carried out with $\mathrm{MBH}$ adducts has allowed the development of a new approach to prepare $\alpha$-hydroxyketones, which have been used to prepare diastereoselective 1,2-anti vicinal aminoalcohols.

Searching to demonstrate the synthetic utility of these approaches, the total synthesis of two pharmacologically active compounds is now described. ( \pm )-Bupropion (1, Figure 1) is an aminoketone acting on CNS. ${ }^{36}$ This compound is commmercialized in its racemic form, since it racemizes very quickly in the body when administered in its enantiomerically pure form. Our target compound could be prepared from acyloin 34 (Figure 3). A 1,2-carbonyl transposition reaction is required to provide $\mathbf{5 9}$, which can be converted uneventfully to $( \pm)-1 .{ }^{37}$

A direct way to perform the required 1,2-carbonyl transposition was using the Lobry-de Bruyn-van Ekenstein transformation..$^{38}$ This reaction, currently used in carbohydrate chemistry, is essentially an enolization of a sugar having a hydrogen at the $\alpha$-carbon to the carbonyl group and proceeds via an enediol intermediate. Some acyloins rearrange with positions exchanged under the influence of base in this transformation. To test this alternative, the TBS group of acyloin 34 was removed by treatment with TBAF in methanol for $12 \mathrm{~h}$ to give the corresponding unprotected acyloin in<smiles>CC(=O)C([O+])c1cccc(Cl)c1</smiles>

$(+/-)-34$

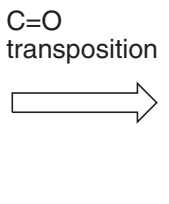

1<smiles>CC(NC(C)(C)C)C(=O)c1cccc(Cl)c1</smiles>

(+/-)-bupropion (1)

Figure 3. Synthesis of ( \pm )-bupropion from acyloin 34 
$75 \%$ yield. A methanol solution of this acyloin was reacted with a $30 \%$ solution of $\mathrm{NaOH}$ at room temperature. As a result, an extensive acyloin degradation was observed even at lower temperatures $\left(-5\right.$ and $\left.-10{ }^{\circ} \mathrm{C}\right)$. We also tested the Voight amination (formation of ketoamine by treatment of an acyloin with $\mathrm{P}_{2} \mathrm{O}_{5}$ in the presence of a primary amine), however this reaction also failed. ${ }^{39}$

Due to these results, the synthetic approach towards the synthesis of bupropion was changed. As an alternative, the required transposition could be done in three steps. Thus, carbonyl reduction of the acyloin ( \pm )-34 directly led to a monoprotected diol $( \pm)-\mathbf{6 0}$ in almost quantitative yield. TBS removal was performed in the presence of TBAF to afford diol $( \pm)-61$ in $97 \%$ yield, as a mixture of diastereoisomers. Selective benzylic oxidation of ( \pm )-61 with IBX in DMSO expectedly led to the 1,2-carbonyl transposition product $( \pm)-\mathbf{5 9}$, as the sole product, in $85 \%$ yield (3 steps, $81 \%$ overall yield, Scheme 4).

Bupropion, in its racemic version, was obtained in 7 steps from Morita-Baylis-Hillman 4, in 27\% overall yield. The sequence is facile to execute and can be scaled-up without problems.

The synthetic methods described in this paper can be combined in order to synthesize spisulosine (2, Figure 1) as a racemate. This natural anti-tumoral compound can be prepared from acyloin $\mathbf{4 2}$, using a diastereoselective reductive amination step (Scheme 5).

The diastereoselective total synthesis of spisulosine was accomplished from acyloin $( \pm)-\mathbf{4 2}$ using a very simple and direct sequence. Reductive amination of acyloin ( \pm )-42 was performed with an excess of benzylamine to give vicinal aminoalcohol ( \pm )-62, with high anti diastereoselectivity (> 95:5, anti:syn), in 68\% yield (Scheme 6). To quickly finish the synthesis, it was only necessary to remove the protecting groups (TBS and benzyl). For thus, a solution of ( \pm )-62 in methanol was acidified with some drops of concentrated $\mathrm{HCl}$ and the mixture was poured into a hydrogenating bottle containing $10 \%$ Pd on charcoal (10 mol\%). To our surprise, after several hours at $60 \mathrm{psi}$, neither of the protecting groups was removed. Perhaps, the long carbon chain favoured the formation of mycelles, which could interact with the solid catalyst.

To solve this unexpected issue, a mixture of solvents for the hydrogenation reaction was used. Thus, a mixture of $( \pm)-62$ in dichloromethane:acetic acid (1.5:1) in the presence of $10 \% \mathrm{Pd} / \mathrm{C}$ at $60 \mathrm{psi}$ and $50{ }^{\circ} \mathrm{C}$ was shaken for $20 \mathrm{~h}$ to furnish the debenzylated aminoalcohol ( \pm )-63 in $87 \%$ yield.

Finally, the TBS group of ( \pm )-63 was removed by the treatment with concentrated $\mathrm{HCl}(0.1 \mathrm{~mL})$ in a solution of dichoromethane:methanol $(1: 1 ; 1 \mathrm{~mL})$ to give $( \pm)$-spisulosine (2) in $98 \%$ yield. All spectroscopic and physical data are identical to those described in literature for natural and synthetic spisulosine (Scheme 6). ${ }^{24,40}$

Spisulosine was synthetized in 7 steps from hexadecanal with an overall yield of $10 \%$. The strategy is very simple and requires no special conditions such as low temperature or dry solvents. If the sequence begins with adduct $\mathbf{1 2}$ in its enantiomerically pure form, it would allow the<smiles>CC(=O)C([18OH])c1cccc(Cl)c1</smiles>

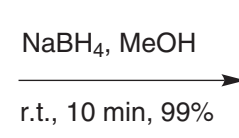

r.t., $10 \mathrm{~min}, 99 \%$

$(+/-)-34$<smiles>CC(O)C(O[Ga+])c1cccc(Cl)c1</smiles>

$(+/-)-60$

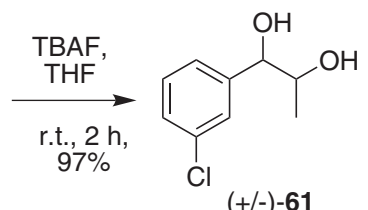

$(+/-)-61$ r.t., 30 h, $85 \%$

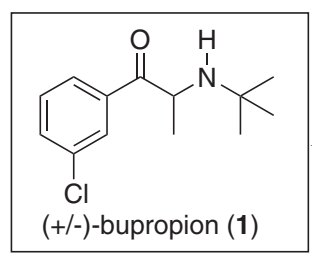<smiles>CC(O)C(=O)c1cccc(Cl)c1</smiles>

Scheme 4. Racemic total synthesis of bupropion from an acyloin.

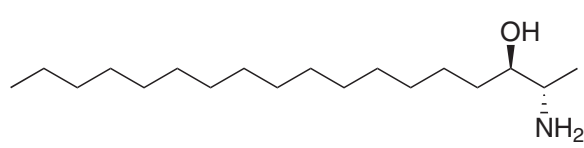

( \pm )-spisulosine (2)

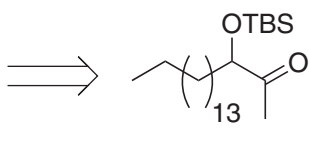

$( \pm)$-acyloin (42)

Scheme 5. Retrosynthesis of spisulosine (2). 


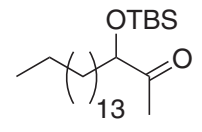

$( \pm)$-acyloin $(42)$

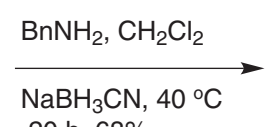

$20 \mathrm{~h}, 68 \%$

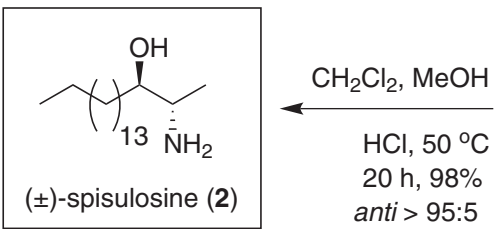

Scheme 6. Diastereoselective total synthesis of (+/-)-spisulosine from acyloin.

enantioselective synthesis of spisulosine. By using this strategy, spisulosine derivatives can also be synthesized using the sequence described herein.

\section{Conclusions}

In summary, the Curtius rearrangement coupled with Morita-Baylis-Hillman adducts as substrate has proven to be an alternative for the preparation of interesting building blocks for organic syntheses. This combination allowed the synthesis of a set of acyloins in good overall yields for three steps. Reductive amination of these acyloins gave vicinal aminoalcohols in good overall yields and with high diastereoselectivities, in favor of the 1,2-anti isomer. Moreover, we have demonstrated that these methods can be used in the synthesis of important pharmacologically active compounds. Thus, ( \pm )-bupropion was prepared in 7 steps from a $\mathrm{MBH}$ adduct, in $27 \%$ overall yield. Using this strategy we also showed a highly diastereoselective total synthesis of $( \pm)$-spisulosine which was accomplished in 7 steps from hexadecanal with an overall yield of $10 \%$.

\section{Experimental}

\section{General procedure}

The ${ }^{1} \mathrm{H}$ and ${ }^{13} \mathrm{C}$ NMR spectra were recorded on a Bruker at 250 and $62.5 \mathrm{MHz}$, respectively, or on an Inova instrument at 500 and $125 \mathrm{MHz}$, respectively. High resolution mass (HRMS) spectra were recorded using a Q-TOF Micromass equipment (Waters, UK). Manipulations and reactions were not performed under dry atmospheres or employing dry solvents, unless otherwise specified. In those cases $\mathrm{CH}_{2} \mathrm{Cl}_{2}$, DMF and triethylamine were dried over $\mathrm{CaH}_{2}$ and distilled. Purification and separations by column chromatography were performed on silica gel, using normal or flash chromatography. Thin layer chromatography (TLC) was

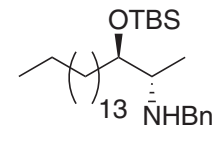

anti $>95: 5$

$( \pm)-(62)$

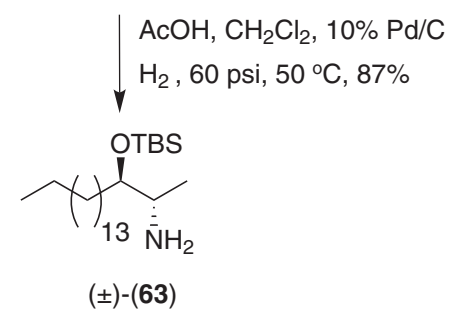

detected by spraying with 5\% ethanolic phosphomolybdic acid and heating. All the Morita-Baylis-Hillman reactions were sonicated in an ultrasonic cleaner (81 W, $40 \mathrm{MHz})$.

General procedure for the synthesis of the Morita-BaylisHillman adducts

A mixture of aldehyde (3-10 mmol), methyl acrylate (excess, ca. 5 equiv.) and DABCO (0.65 equiv.) was sonicated in an ultrasound bath at $30^{\circ} \mathrm{C}$. Reaction evolution was followed by TLC analysis. For the preparation of the $\mathrm{MBH}$ adducts $\mathbf{1 0}$ and $\mathbf{1 2}$ the reaction was carried out using methyl acrylate (10 equiv.), ionic liquid ([bmim $] \mathrm{BF}_{4} ; 5$ drops) as an additive, at $50^{\circ} \mathrm{C}$ with stirring. The excess of methyl acrylate was removed under vaccum. The crude residue was diluted in ethyl acetate $(25 \mathrm{~mL})$. The organic layer was washed with distilled water $(15 \mathrm{~mL})$, brine $(2 \times 15 \mathrm{~mL})$, dried over anhydrous $\mathrm{Na}_{2} \mathrm{SO}_{4}$ and the solvent was removed under vacuum. The residue was purified by flash silica gel column chromatography (ethyl acetate:hexanes, up to 30:70) to provide the MBH adducts in good to high yields.

(士)-Methyl 2-[hydroxy(2-nitrophenyl)methyl]prop2-enoate (3): 92\% yield, pale yellow oil; IR (film) $\mathrm{v}_{\max } / \mathrm{cm}^{-1}: 3469,1716,1630,1528,1352 ;{ }^{1} \mathrm{H}$ NMR $\left(500 \mathrm{MHz}, \mathrm{CDCl}_{3}\right): \delta 7.94(\mathrm{dd}, J 1.2 / 8.2 \mathrm{~Hz}, 1 \mathrm{H}), 7.78(\mathrm{~d}$, 1H, J 7.9 Hz), 7.61-7.67 (m, 1H), 7.41-7.48 (m, 1H), 6.36 $(\mathrm{s}, 1 \mathrm{H}), 6.20(\mathrm{~s}, 1 \mathrm{H}), 5.72(\mathrm{~s}, 1 \mathrm{H}), 3.73(\mathrm{~s}, 3 \mathrm{H}), 3.08(\mathrm{~s}$, $1 \mathrm{H}, \mathrm{OH}) ;{ }^{13} \mathrm{C}$ NMR $\left(125 \mathrm{MHz}, \mathrm{CDCl}_{3}\right): \delta 166.3,148.2$, 140.7, 136.0, 133.4, 128.8, 128.6, 126.4, 124.5, 67.6, 52.1; HRMS (ESI TOF) Calcd. for $\mathrm{C}_{11} \mathrm{H}_{11} \mathrm{NO}_{5}\left[\mathrm{M}+\mathrm{Na}^{+}\right]$: 260.0529. Found: 260.0530 .

(士)-Methyl 2-[(3-chlorophenyl)(hydroxy)methyl] prop-2-enoate (4): $89 \%$ yield, colorless oil; IR (film) $\mathrm{v}_{\max } / \mathrm{cm}^{-1}: 3452,1711,1433,1282,1151,1041 ;{ }^{1} \mathrm{H} \mathrm{NMR}$ 
(250 MHz, $\left.\mathrm{CDCl}_{3}\right): \delta 7.36(\mathrm{~s}, 1 \mathrm{H}), 7.21-7.26(\mathrm{~m}, 3 \mathrm{H}), 6.34$ (s, 1H), $5.84(\mathrm{~s}, 1 \mathrm{H}), 5.49(\mathrm{~s}, 1 \mathrm{H}), 3.71(\mathrm{~s}, 3 \mathrm{H}), 3.16(\mathrm{~s}$, $1 \mathrm{H}, \mathrm{OH}) ;{ }^{13} \mathrm{C}$ NMR $\left(62.5 \mathrm{MHz}, \mathrm{CDCl}_{3}\right): \delta 166.6,143.4$, 141.4, 134.3, 129.7, 127.9, 126.7, 124.8, 72.6, 52.1; HRMS (ESI TOF) Calcd. for $\mathrm{C}_{11} \mathrm{H}_{11} \mathrm{O}_{3} \mathrm{Cl}\left[\mathrm{M}+\mathrm{Na}^{+}\right]$: 249.0294 . Found: 249.0318.

( \pm )-Methyl 2-[hydroxy(phenyl)methyl]prop-2-enoate (5): 75\% yield, oil; IR (film) $v_{\max } / \mathrm{cm}^{-1}: 3448,2949,1711$, 1446, 1270, 1155, 1041; ${ }^{1} \mathrm{H}$ NMR (250 MHz, $\mathrm{CDCl}_{3}$ ): $\delta$ 7.21-7.37 (m, 5H), $6.31(\mathrm{~s}, 1 \mathrm{H}), 5.86(\mathrm{~s}, 1 \mathrm{H}), 5.52(\mathrm{~s}$, $1 \mathrm{H}), 3.65$ (s, 3H), 3.45 (s, 1H, OH); ${ }^{13} \mathrm{C}$ NMR $(62.5 \mathrm{MHz}$, $\left.\mathrm{CDCl}_{3}\right): \delta 166.7,142.1,141.4,128.4,127.8,126.7$, 125.8, 72.8, 51.9; HRMS (ESI TOF) Calcd. for $\mathrm{C}_{11} \mathrm{H}_{12} \mathrm{O}_{3}$ $\left[\mathrm{M}+\mathrm{Na}^{+}\right]:$215.0684. Found: 215.0811 .

( \pm )-Methyl 2-[hydroxy(4-methoxyphenyl)methyl]prop2-enoate (6): $73 \%$ yield; amorphous solid; $\mathrm{mp} 70-72{ }^{\circ} \mathrm{C}$; IR (KBr) $v_{\text {max }} / \mathrm{cm}^{-1}: 3465,1714,1611,1512,1465,1034$; ${ }^{1} \mathrm{H}$ NMR $\left(250 \mathrm{MHz}, \mathrm{CDCl}_{3}\right): \delta 7.29(\mathrm{~d}, 2 \mathrm{H}, J 8.5 \mathrm{~Hz})$, $6.87(\mathrm{~d}, 2 \mathrm{H}, J 8.5 \mathrm{~Hz}), 6.32(\mathrm{~s}, 1 \mathrm{H}), 5.85(\mathrm{~s}, 1 \mathrm{H}), 5.53(\mathrm{~s}$, $1 \mathrm{H}), 3.80(\mathrm{~s}, 3 \mathrm{H}), 3.72(\mathrm{~s}, 3 \mathrm{H}), 2.43(\mathrm{~s}, 1 \mathrm{H}, \mathrm{OH}) ;{ }^{13} \mathrm{C} \mathrm{NMR}$ $\left(62.5 \mathrm{MHz}, \mathrm{CDCl}_{3}\right): \delta 166.8,159.2,142.1,133.4,127.9$, 125.7, 113.8, 72.8, 55.2, 51.9; HRMS (ESI TOF) Calcd. for $\mathrm{C}_{12} \mathrm{H}_{14} \mathrm{O}_{4}\left[\mathrm{M}+\mathrm{Na}^{+}\right]: 245.0784$. Found: 245.0780 .

( \pm -Methyl 2-[hydroxy(3,4,5-trimethoxyphenyl)methyl] prop-2-enoate (7): $60 \%$ yield; amorphous solid; mp 121$123^{\circ} \mathrm{C}$; IR (KBr) $v_{\text {max }} / \mathrm{cm}^{-1}: 3464,1712,1613,1514,1463$, 1036; ${ }^{1} \mathrm{H}$ NMR (250 MHz, $\left.\mathrm{CDCl}_{3}\right): \delta 6.54(\mathrm{~s}, 2 \mathrm{H}), 6.27$ $(\mathrm{s}, 1 \mathrm{H}), 5.81(\mathrm{~s}, 1 \mathrm{H}), 5.44(\mathrm{~d}, 1 \mathrm{H}, J 5.0 \mathrm{~Hz}), 3.78(\mathrm{~s}, 6 \mathrm{H})$, 3.77 (s, 3H), 3.69 (s, 3H), 3.35 (d, 1H, J 5.0 Hz); ${ }^{13} \mathrm{C}$ NMR $\left(62.5 \mathrm{MHz}, \mathrm{CDCl}_{3}\right): \delta 166.8,153.1,141.9,137.2,137.0$, 125.9, 103.5, 72.9, 60.7, 55.9, 52.0; HRMS (ESI TOF) Calcd. for $\mathrm{C}_{11} \mathrm{H}_{11} \mathrm{O}_{3} \mathrm{Cl}\left[\mathrm{M}+\mathrm{Na}^{+}\right]$: 305.0996. Found: 305.0989.

(士)-Methyl 2-[hydroxy(thiophen-2-yl)methyl]prop2-enoate (8): $90 \%$ yield; pale yellow oil; IR (film) $v_{\max } / \mathrm{cm}^{-1}$ : $3445,2952,1715,1632 ;{ }^{1} \mathrm{H} \mathrm{NMR}\left(250 \mathrm{MHz}, \mathrm{CDCl}_{3}\right): \delta 7.21$ (dd, 1H, J2.5/3.8 Hz), 6.90-6.93 (m, 2H), 6.32 (s, 1H), 5.95 (s, 1H), $5.73(\mathrm{~d}, 1 \mathrm{H}, J 6,2 \mathrm{~Hz}), 3.73(\mathrm{~d}, 1 \mathrm{H}, J 6.2 \mathrm{~Hz}), 3.70$ (s, 3H); ${ }^{13} \mathrm{C} \mathrm{NMR}\left(62.5 \mathrm{MHz}, \mathrm{CDCl}_{3}\right): \delta 166.5,145.8,141.5$, 126.8, 126.0, 125.2, 124.8, 69.1, 52.0; HRMS (ESI TOF) Calcd. for $\mathrm{C}_{9} \mathrm{H}_{11} \mathrm{O}_{3} \mathrm{~S}[\mathrm{M}+\mathrm{H}]^{+}$: 199.0423. Found: 199.0417.

( \pm )-Methyl 2-[(2-fluorophenyl)(hydroxy)methyl]prop2-enoate (9): 95\% yield; colorless oil; IR (film) $v_{\max } / \mathrm{cm}^{-1}$. 3437, 1721; ${ }^{1} \mathrm{H}$ NMR $\left(250 \mathrm{MHz}, \mathrm{CDCl}_{3}\right): \delta 7.42(\mathrm{dt}, 1 \mathrm{H}$, $J 1.9 / 7.5 \mathrm{~Hz}), 7.21-7.28(\mathrm{~m}, 1 \mathrm{H}), 7.10(\mathrm{dt}, 1 \mathrm{H}, J 1.2 / 7.5 \mathrm{~Hz})$, 6.97-7.05 (m, 1H), $6.31(\mathrm{~s}, 1 \mathrm{H}), 5.85(\mathrm{~d}, 1 \mathrm{H}, J 5.5 \mathrm{~Hz}), 5.76$ (s, 1H), 3.69 (s, 3H), 3.62 (d, 1H, J 5.5 Hz); ${ }^{13} \mathrm{C}$ NMR (62.5
$\mathrm{MHz}, \mathrm{CDCl}_{3}$ ): $\delta 166.7,160.0(\mathrm{~d}, J 245.5 \mathrm{~Hz}), 140.9,129.4$ (d, $J 8.2 \mathrm{~Hz}), 128.4,128.1$ (d, $J 3.7 \mathrm{~Hz}), 126.3,124.1$ (d, $J$ $3.5 \mathrm{~Hz}), 115.3$ (d, $J 21.5 \mathrm{~Hz}), 66.7,52.0$; HRMS (ESI TOF) Calcd. for $\mathrm{C}_{11} \mathrm{H}_{12} \mathrm{FO}_{3}[\mathrm{M}+\mathrm{H}]^{+}:$211.0765. Found: 211.0762.

(士)-Methyl 3-hydroxy-2-methylenepentanoate (10): $80 \%$ yield; colorless oil; IR (film) $v_{\max } / \mathrm{cm}^{-1}: 3454,2928$, 2853, 1705, 1287, 1157; ${ }^{1} \mathrm{H}$ NMR (250 MHz, $\left.\mathrm{CDCl}_{3}\right)$ : $\delta 6.15(\mathrm{~s}, 1 \mathrm{H}), 5.74(\mathrm{~s}, 1 \mathrm{H}), 4.27(\mathrm{q}, 1 \mathrm{H}, J 5.7 \mathrm{~Hz}), 3.69(\mathrm{~s}$, $3 \mathrm{H}), 3.00(\mathrm{~d}, 1 \mathrm{H}, J 5.7 \mathrm{~Hz}), 1.45-1.71(\mathrm{~m}, 2 \mathrm{H}), 0.86(\mathrm{t}, 3 \mathrm{H}$, $J 7.4 \mathrm{~Hz}) ;{ }^{13} \mathrm{C}$ NMR $\left(62.5 \mathrm{MHz}, \mathrm{CDCl}_{3}\right): \delta 167.0,142.3$, 124.9, 72.4, 51.7, 29.0, 9.9; HRMS (ESI TOF) Calcd. for $\mathrm{C}_{7} \mathrm{H}_{13} \mathrm{O}_{3}[\mathrm{M}+\mathrm{H}]^{+}:$145.0859. Found: 145.0856 .

( \pm )-Methyl 3-hydroxy-2-methylenenonanoate (11): $76 \%$ yield; amorphous solid; mp $131-132{ }^{\circ} \mathrm{C}$; IR (KBr) $v_{\max } / \mathrm{cm}^{-1}: 3452,2929,2855,1707,1290,1155 ;{ }^{1} \mathrm{H}$ NMR $\left(500 \mathrm{MHz}, \mathrm{CDCl}_{3}\right): \delta 6.20(\mathrm{~s}, 1 \mathrm{H}), 5.78(\mathrm{~s}, 1 \mathrm{H}), 4.37(\mathrm{t}$, $1 \mathrm{H}, J 6.7 \mathrm{~Hz}$ ), 3.77 (s, 3H), 1.54-1.71 (m, 2H), 1.19-1.50 (m, 8H), 0.86 (t, 3H, J $6.7 \mathrm{~Hz}) ;{ }^{13} \mathrm{C}$ NMR $(125 \mathrm{MHz}$, $\left.\mathrm{CDCl}_{3}\right): \delta 167.0,142.5,124.9,71.7,51.8,36.2,31.7,29.0$, 25.7, 22.5, 14.0; HRMS (ESI TOF) Calcd. for $\mathrm{C}_{11} \mathrm{H}_{21} \mathrm{O}_{3}$ $[\mathrm{M}+\mathrm{H}]^{+}:$201.1485. Found: 201.1483.

(士)-Methyl-3-hydroxy-2-methyleneoctadecanoate (12): $60 \%$ yield; amorphous solid; mp $43-45^{\circ} \mathrm{C}$; IR (film) $v_{\text {max }} / \mathrm{cm}^{-1}: 3465,2984,2929,2856,1742,1374,1242,1048$; ${ }^{1} \mathrm{H}$ NMR $\left(250 \mathrm{MHz}, \mathrm{CDCl}_{3}\right): \delta 6.21(\mathrm{~s}, 1 \mathrm{H}), 5.79(\mathrm{t}, 1 \mathrm{H}$, $J 1.0 \mathrm{~Hz}), 4.37(\mathrm{q}, 1 \mathrm{H}, J 6.5 \mathrm{~Hz}), 3.77(\mathrm{~s}, 3 \mathrm{H}), 2.59(\mathrm{~d}, 1 \mathrm{H}$, $J 6.5 \mathrm{~Hz}), 1.55-1.70(\mathrm{~m}, 2 \mathrm{H}), 1.18-1.50(\mathrm{~m}, 26 \mathrm{H}), 0.87(\mathrm{t}$, $3 \mathrm{H}, J 6.9 \mathrm{~Hz}) ;{ }^{13} \mathrm{C}$ NMR $\left(62.5 \mathrm{MHz}, \mathrm{CDCl}_{3}\right): \delta 167,142.5$, 124.9, 71.8, 51.8, 36.2, 31.9, 29.7, 29.63, 29.6, 29.5, 29.4, 29.3, 25.8, 22.7, 14.1; HRMS (ESI) Calcd. for $\mathrm{C}_{20} \mathrm{H}_{39} \mathrm{O}_{3}$ $[\mathrm{M}+\mathrm{H}]^{+}$327.2894; Found 327.2877.

General procedure for the silylation of Morita-BaylisHillman adducts

A mixture of $\mathrm{MBH}$ adduct (1-3 mmol), imidazole (2.5 equiv.), $\mathrm{TBSCl}$ ( 1.5 equiv.) and a few drops of DMSO (to facilitate stirring), under argon atmosphere, was stirred at room temperature. After 2-4 h, the crude residue was diluted in ethyl acetate $(25 \mathrm{~mL})$. The organic layer was washed with distilled water $(15 \mathrm{~mL})$, brine $(2 \times 15 \mathrm{~mL})$, dried over anhydrous $\mathrm{Na}_{2} \mathrm{SO}_{4}$ and the solvent was removed under vacuum. The crude mixture was filtered through silica gel (ethyl acetate:hexanes, 30:70) to provide the silylated compounds in good to excellent yields.

( \pm )-Methyl 2-\{[(tert-butyldimethylsilyl)oxy](2-fluorophenyl)methyl]prop-2-enoate (13): 97\% yield; pale yellow 
oil; IR (film) $v_{\max } / \mathrm{cm}^{-1}: 2949,2929,2851,1728,1531,1352$, $1249,1090,837 ;{ }^{1} \mathrm{H}$ NMR $\left(250 \mathrm{MHz}, \mathrm{CDCl}_{3}\right): \delta 7.78(\mathrm{~d}$, $1 \mathrm{H}, J 8.0 \mathrm{~Hz}), 7.68(\mathrm{~d}, 1 \mathrm{H}, J 8.0 \mathrm{~Hz}), 7.56(\mathrm{t}, 1 \mathrm{H}, J 7.5 \mathrm{~Hz})$, $7.37(\mathrm{t}, 1 \mathrm{H}, J 7.5 \mathrm{~Hz}), 6.34(\mathrm{~s}, 1 \mathrm{H}), 6.29(\mathrm{~s}, 1 \mathrm{H}), 5.97(\mathrm{~s}$, $1 \mathrm{H}), 3.64(\mathrm{~s}, 3 \mathrm{H}), 0.88(\mathrm{~s}, 9 \mathrm{H}), 0.13(\mathrm{~s}, 3 \mathrm{H}),-0.06(\mathrm{~s}, 3 \mathrm{H})$; ${ }^{13} \mathrm{C} \mathrm{NMR}\left(62.5 \mathrm{MHz}, \mathrm{CDCl}_{3}\right): \delta 165.2,148.2,143.1,137.5$, 132.6, 129.6, 128.1, 125.0, 123.9, 66.7, 51.8, 25.7, 18.1, -5.1. HRMS (ESI TOF) Calcd. for $\mathrm{C}_{17} \mathrm{H}_{25} \mathrm{NO}_{5} \mathrm{Si}\left[\mathrm{M}+\mathrm{Na}^{+}\right]$: 374.1394. Found: 374.1389.

( \pm -Methyl 2-\{[(tert-butyldimethylsilyl)oxy](3-chlorophenyl)methyl\}prop-2-enoate (14): > 99\% yield; pale yellow oil; IR (film) $v_{\max } / \mathrm{cm}^{-1}: 2949,2929,2855,1707$, 1061, 841, 780; ${ }^{1} \mathrm{H}$ NMR $\left(250 \mathrm{MHz}, \mathrm{CDCl}_{3}\right.$ ): $\delta 7.35$ (s, $1 \mathrm{H}), 7.17-7.32(\mathrm{~m}, 3 \mathrm{H}), 6.28(\mathrm{~s}, 1 \mathrm{H}), 6.10(\mathrm{~s}, 1 \mathrm{H}), 5.58$ $(\mathrm{s}, 1 \mathrm{H}), 3.69(\mathrm{~s}, 3 \mathrm{H}), 0.88(\mathrm{~s}, 9 \mathrm{H}), 0.06(\mathrm{~s}, 3 \mathrm{H}),-0.08(\mathrm{~s}$, $3 \mathrm{H}) ;{ }^{13} \mathrm{C}$ NMR $\left(75.5 \mathrm{MHz}, \mathrm{CDCl}_{3}\right): \delta 166.1,144.8,143.4$, 133.9, 129.3, 127.5, 127.0, 125.2, 124.4, 72.1, 51.7, 25.7, 18.1, -5.0; HRMS (ESI TOF) Calcd. for $\mathrm{C}_{17} \mathrm{H}_{25} \mathrm{ClO}_{3} \mathrm{Si}$ $\left[\mathrm{M}+\mathrm{Na}^{+}\right]: 363.1154$. Found: 363.1150 .

(士)-Methyl 2-\{[(tert-butyldimethylsilyl)oxy](phenyl) methyl'prop-2-enoate (15): > 99\% yield; colorless oil; IR (film) $v_{\max } / \mathrm{cm}^{-1}: 2949,2851,1458,1258,1082 ;{ }^{1} \mathrm{H}$ NMR $\left(250 \mathrm{MHz}, \mathrm{CDCl}_{3}\right): \delta$ 7.17-7.41 (m, 5H), $6.25(\mathrm{~s}, 1 \mathrm{H}), 6.08$ $(\mathrm{s}, 1 \mathrm{H}), 5.61(\mathrm{~s}, 1 \mathrm{H}), 3.68(\mathrm{~s}, 3 \mathrm{H}), 0.88(\mathrm{~s}, 9 \mathrm{H}), 0.06(\mathrm{~s}$, $3 \mathrm{H}),-0.11(\mathrm{~s}, 3 \mathrm{H}) ;{ }^{13} \mathrm{C} \mathrm{NMR}\left(62.5 \mathrm{MHz}, \mathrm{CDCl}_{3}\right): \delta 166.4$, 143.9, 142.6, 128.0, 127.3, 127.0, 123.8, 72.7, 51.6, 25.7, 18.1, -4.9; HRMS (ESI TOF) Calcd. for $\mathrm{C}_{17} \mathrm{H}_{26} \mathrm{O}_{3} \mathrm{Si}[\mathrm{M}+$ $\left.\mathrm{Na}^{+}\right]$: 329.1543 . Found: 329.1537.

(士)-Methyl 2-\{[(tert-butyldimethylsilyl)oxy] (4-methoxyphenyl)methyl\}prop-2-enoate (16): > 99\% yield; amorphous solid; mp 112-114 ${ }^{\circ} \mathrm{C}$; IR (KBr) $v_{\max } / \mathrm{cm}^{-1}$ : $2949,2929,2851,1715,1507,1245,1047,833,771 ;{ }^{1} \mathrm{H}$ NMR (250 MHz, $\left.\mathrm{CDCl}_{3}\right): \delta 7.27(\mathrm{~d}, 2 \mathrm{H}, J 7.0 \mathrm{~Hz}), 6.82(\mathrm{~d}$, $2 \mathrm{H}, J 7.0 \mathrm{~Hz}), 6.22(\mathrm{~s}, 1 \mathrm{H}), 6.06(\mathrm{~s}, 1 \mathrm{H}), 5.56(\mathrm{~s}, 1 \mathrm{H}), 3.78$ (s, 3H), 3.67 (s, 3H), 0.87 (s, 9H), $0.06(\mathrm{~s}, 3 \mathrm{H}),-0.11$ (s, $3 \mathrm{H}) ;{ }^{13} \mathrm{C}$ NMR $\left(125 \mathrm{MHz}, \mathrm{CDCl}_{3}\right): \delta 166.4,158.8,144.1$, 134.8, 128.2, 123.3, 113.4, 72.3, 55.1, 51.6, 25.7, 18.1, -4.9; HRMS (ESI TOF) Calcd. for $\mathrm{C}_{18} \mathrm{H}_{28} \mathrm{O}_{4} \mathrm{Si}\left[\mathrm{M}+\mathrm{Na}^{+}\right]$: 359.1649. Found: 359.1647.

(土)-Methyl 2-\{[(tert-butyldimethylsilyl)oxy] (3,4,5-trimethoxyphenyl)methyl\}prop-2-enoate (17): 80\% yield; colorless oil; IR (film) $v_{\max } / \mathrm{cm}^{-1}: 2950,2927,2854$, 1718, 1505, 1246, 1051; ${ }^{1} \mathrm{H}$ NMR (250 MHz, $\left.\mathrm{CDCl}_{3}\right)$ : $\delta 6.56(\mathrm{~s}, 2 \mathrm{H}), 6.18(\mathrm{t}, 1 \mathrm{H}, J 1.1 \mathrm{~Hz}), 5.95(\mathrm{t}, 1 \mathrm{H}, J 1.5 \mathrm{~Hz})$, $5.53(\mathrm{~s}, 1 \mathrm{H}), 3.78(\mathrm{~s}, 6 \mathrm{H}), 3.67(\mathrm{~s}, 3 \mathrm{H}), 0.85(\mathrm{~s}, 9 \mathrm{H}), 0.03(\mathrm{~s}$, $3 \mathrm{H}),-0.10(\mathrm{~s}, 3 \mathrm{H}) ;{ }^{13} \mathrm{C}$ NMR $\left(62.5 \mathrm{MHz}, \mathrm{CDCl}_{3}\right): \delta 166.5$, $152.8,143.9,138.3,137.0,123.9,103.7,72.4,60.7,55.9$,
51.6, 25.6, 18.1, -4.9, -5.1; HRMS (ESI TOF) Calcd. for $\mathrm{C}_{20} \mathrm{H}_{32} \mathrm{O}_{6} \mathrm{Si}\left[\mathrm{M}+\mathrm{Na}^{+}\right]$: 419.1860. Found: 419.1855 .

( \pm )-Methyl 2-\{[(tert-butyldimethylsilyl)oxy](thiophen2-yl)methyllprop-2-enoate (18): $90 \%$ yield; colorless oil; IR (film) $v_{\max } / \mathrm{cm}^{-1}: 2954,2930,2857,1723,1632$, 1256,$1082 ;{ }^{1} \mathrm{H}$ NMR $\left(250 \mathrm{MHz}, \mathrm{CDCl}_{3}\right): \delta 7.18(\mathrm{dd}, 1 \mathrm{H}$, $J 5.0 / 1.3 \mathrm{~Hz}), 6.83-6.98$ (m, 2H), 6.28 (t, 1H, J 1.1 Hz), 6.12 (t, 1H, J 1.5 Hz), 5.89 (s, 1H), $3.73(\mathrm{~s}, 3 \mathrm{H}), 0.90(\mathrm{~s}, 9 \mathrm{H})$, $0.08(\mathrm{~s}, 3 \mathrm{H}),-0.003$ (s, 3H); ${ }^{13} \mathrm{C} \mathrm{NMR}\left(62.5 \mathrm{MHz}, \mathrm{CDCl}_{3}\right.$ ): $\delta 166.2,147.3,143.5,126.3,124.6,124.4,124.2,68.4$, 51.7, 25.7, 18.2, -5.1, -5.14; HRMS (ESI TOF) Calcd. for $\mathrm{C}_{15} \mathrm{H}_{24} \mathrm{O}_{3} \mathrm{SSi}\left[\mathrm{M}+\mathrm{Na}^{+}\right]$: 335.1108. Found: 335.1107.

( \pm )-Methyl 2-\{[(tert-butyldimethylsilyl)oxy](2-fluorophenyl)methyl\}prop-2-enoate (19): 85\% yield; colorless oil; IR (film) $v_{\max } / \mathrm{cm}^{-1}: 1723 ;{ }^{1} \mathrm{H} \mathrm{NMR}\left(250 \mathrm{MHz}, \mathrm{CDCl}_{3}\right)$ : $\delta 7.35(\mathrm{dt}, 1 \mathrm{H}, J 1.5 / 7.5 \mathrm{~Hz}), 7.16-7.28(\mathrm{~m}, 1 \mathrm{H}), 6.95-7.14$ $(\mathrm{m}, 2 \mathrm{H}), 6.34(\mathrm{~s}, 1 \mathrm{H}), 6.07(\mathrm{~s}, 1 \mathrm{H}), 5.95(\mathrm{~s}, 1 \mathrm{H}), 3.66(\mathrm{~s}$, $3 \mathrm{H}), 0.87$ (s, 9H), 0.08 (s, 3H), -0.09 (s, 3H); ${ }^{13} \mathrm{C}$ NMR $\left(62.5 \mathrm{MHz}, \mathrm{CDCl}_{3}\right): \delta 166.0,159.8$ (d, $\left.J 245.7 \mathrm{~Hz}\right), 142.7$, 129.5 (d, $J 13.5 \mathrm{~Hz}), 129.1,129.0$ (d, $J 6.8 \mathrm{~Hz}), 128.8$, 124.8, 123.9 (d, J 3.5 Hz), 115.2 (d, J $22.2 \mathrm{~Hz}), 65.8,51.6$, $25.7,18.1,-5.2,-5.3$.

(士)-Methyl 3-[(tert-butyldimethylsilyl)oxy]2-methylidenepentanoate (20): $85 \%$ yield; colorless oil; IR (film) $v_{\text {max }} / \mathrm{cm}^{-1}: 2930,2845,1721 ;{ }^{1} \mathrm{H}$ NMR $(250 \mathrm{MHz}$, $\left.\mathrm{CDCl}_{3}\right): \delta 6.20-6.23(\mathrm{~m}, 1 \mathrm{H}), 5.86-5.89(\mathrm{~m}, 1 \mathrm{H}), 4.50-4.59$ (m, 1H), $3.74(\mathrm{~s}, 3 \mathrm{H}), 1.35-1.75(\mathrm{~m}, 2 \mathrm{H}), 0.90(\mathrm{~s}, 9 \mathrm{H}), 0.85$ (t, 3H, J 7.4 Hz), 0.05 (s, 3H), -0.02 (s, 3H); ${ }^{13} \mathrm{C}$ NMR $\left(62.5 \mathrm{MHz}, \mathrm{CDCl}_{3}\right): \delta 166.8,143.6,124.5,71.0,51.6$, 30.3, 25.8, 18.1, 9.0, -4.8, -5.1; HRMS (ESI TOF) Calcd. for $\mathrm{C}_{17} \mathrm{H}_{25} \mathrm{FO}_{3} \mathrm{Si}\left[\mathrm{M}+\mathrm{Na}^{+}\right.$]: 347.1449 Found: 347.1445.

(士)-Methyl 3-[(tert-butyldimethylsilyl)oxy]2-methylidenenonanoate (21): > 99\% yield; amorphous solid; mp 102-103 ${ }^{\circ} \mathrm{C}$; IR (film) $v_{\max } / \mathrm{cm}^{-1}: 2929,2859$, 1719, 1249, 1090, 837; ${ }^{1} \mathrm{H}$ NMR (250 $\left.\mathrm{MHz}, \mathrm{CDCl}_{3}\right)$ : $\delta$ 6.17-6.21 (m, 1H), 5.87-5.90 (m, 1H), 4.52-4.61 (m, $1 \mathrm{H}), 3.72(\mathrm{~s}, 3 \mathrm{H}), 1.18-1.65(\mathrm{~m}, 10 \mathrm{H}), 0.78-0.92(\mathrm{~m}, 12 \mathrm{H})$, $0.04(\mathrm{~s}, 3 \mathrm{H}),-0.04$ (s, 3H); ${ }^{13} \mathrm{C} \mathrm{NMR}\left(62.5 \mathrm{MHz}, \mathrm{CDCl}_{3}\right)$ : $\delta$ 166.7, 144.1, 124.2, 70.2, 51.5, 37.8, 31.8, 29.1, 25.7, 24.9, 22.6, 18.1, 14.0, -4.8, -5.1; HRMS (ESI TOF) Calcd. for $\mathrm{C}_{17} \mathrm{H}_{34} \mathrm{O}_{3} \mathrm{Si}\left[\mathrm{M}+\mathrm{Na}^{+}\right]$: 337.2169 . Found: 337.2168.

( \pm )-Methyl-3-[(tert-butyldimethylsilyl)oxy]2-methyleneoctadecanoate (22): 70\% yield; viscous colorless oil; IR (film) $v_{\max } / \mathrm{cm}^{-1}: 2926,2855,1722,1463$, 1257,1092 ; ${ }^{1} \mathrm{H}$ NMR $\left(250 \mathrm{MHz}, \mathrm{CDCl}_{3}\right): \delta 6.19-6.21(\mathrm{~m}$, $1 \mathrm{H}), 5.90(\mathrm{t}, 1 \mathrm{H}, J 1.6 \mathrm{~Hz}), 4.56-4.61(\mathrm{~m}, 1 \mathrm{H}), 3.75(\mathrm{~s}, 3 \mathrm{H})$, 
$1.18-1.63(\mathrm{~m}, 28 \mathrm{H}), 0.81-0.93(\mathrm{~m}, 12 \mathrm{H}), 0.05(\mathrm{~s}, 3 \mathrm{H}),-0.02$ (s, 3H); ${ }^{13} \mathrm{CNMR}\left(62.5 \mathrm{MHz}, \mathrm{CDCl}_{3}\right): \delta 166.7,144.2,124.2$, 70.2, 51.6, 37.9, 31.9, 29.7, 29.63, 29.6, 29.55, 29.5, 29.3, 25.8, 22.7, 18.1, 14.1, -4.8, -5.1; HRMS (ESI TOF) Calcd. for $\mathrm{C}_{26} \mathrm{H}_{52} \mathrm{O}_{3} \mathrm{Si}[\mathrm{M}+\mathrm{Na}]^{+}:$463.3578. Found: 463.3572 .

\section{General procedure for hydrolysis of silylated esters}

To a solution of silylated MBH adduct (1-3 mmol) in a mixture of water:acetonitrile (1:1) was added $\mathrm{LiOH}$ (10 equiv.). The resulting solution was stirred for $4 \mathrm{~h}$ at $50-60{ }^{\circ} \mathrm{C}$. Then, the solvents were removed under reduced pressure and the crude mixture was extracted with ethyl acetate. The organic layer was dried over anhydrous $\mathrm{Na}_{2} \mathrm{SO}_{4}$ and the solvent was removed under vacuum. The residue was filtered through silica gel (ethyl acetate as solvent) to provide the corresponding carboxylic acids in almost quantitative yields, for most cases $>99 \%$.

(士)-2-\{[(tert-Butyldimethylsilyl)oxy](2-nitrophenyl) methyl]prop-2-enoic acid (23): 97\% yield; viscous pale yellow oil; IR (film) $v_{\max } / \mathrm{cm}^{-1}: 2945,2864,2353,1703,1531$, $1258,1090,837 ;{ }^{1} \mathrm{H}$ NMR $\left(250 \mathrm{MHz}, \mathrm{CDCl}_{3}\right): \delta 7.81(\mathrm{~d}$, $1 \mathrm{H}, J 8.0 \mathrm{~Hz}), 7.71(\mathrm{~d}, 1 \mathrm{H}, J 8.0 \mathrm{~Hz}), 7.57(\mathrm{t}, 1 \mathrm{H}, J 7.5 \mathrm{~Hz})$, $7.38(\mathrm{t}, 1 \mathrm{H}, J 7.5 \mathrm{~Hz}), 6.40(\mathrm{~s}, 1 \mathrm{H}), 6.34(\mathrm{~s}, 1 \mathrm{H}), 6.04(\mathrm{~s}, 1 \mathrm{H})$, 0.90 (s, 9H), 0.13 (s, 3H), 0.05 (s, 3H); ${ }^{13} \mathrm{C}$ NMR $(62.5 \mathrm{MHz}$, $\left.\mathrm{CDCl}_{3}\right): \delta 170.4,148.1,142.4,137.3,132.7,129.5,128.2$, 127.2, 124.0, 66.6, 25.7, 18.1, -4.9; HRMS (ESI TOF) Calcd. for $\mathrm{C}_{16} \mathrm{H}_{23} \mathrm{NO}_{5} \mathrm{Si}\left[\mathrm{M}+\mathrm{Na}^{+}\right]$: 360.1238. Found: 360.1237.

( \pm )-2-\{[(tert-Butyldimethylsilyl)oxy](3-chlorophenyl) methyl\}prop-2-enoic acid (24): 99\% yield; viscous colorless oil; IR (film) $v_{\text {max }} / \mathrm{cm}^{-1}: 2949,2929,2851,1691,1437,1254$, $1074,833,780 ;{ }^{1} \mathrm{H} \mathrm{NMR}\left(250 \mathrm{MHz}, \mathrm{CDCl}_{3}\right): \delta 7.34(\mathrm{~s}, 1 \mathrm{H})$, 7.18-7.29 (m, 3H), $6.43(\mathrm{~s}, 1 \mathrm{H}), 6.19(\mathrm{~s}, 1 \mathrm{H}), 5.54(\mathrm{~s}, 1 \mathrm{H})$, 0.88 (s, 9H), 0.06 (s, 3H), -0.07 (s, 3H); ${ }^{13} \mathrm{C}$ NMR (62.5 $\left.\mathrm{MHz}, \mathrm{CDCl}_{3}\right): \delta 170.1,144.4,142.6,134.0,129.4,127.7$, 127.0, 125.1, 71.9, 25.7, 18.1, -5.0; HRMS (ESI TOF) Calcd. for $\mathrm{C}_{16} \mathrm{H}_{23} \mathrm{O}_{3} \mathrm{ClSi}\left[\mathrm{M}+\mathrm{Na}^{+}\right]$: 349.0918. Found: 349.1003.

(土)-2-\{[(tert-Butyldimethylsilyl)oxy](phenyl)methyl\} prop-2-enoic acid (25): $99 \%$ yield; yellowish oil; ${ }^{1} \mathrm{H}$ NMR (250 MHz, $\mathrm{CDCl}_{3}$ ): $\delta$ 7.20-7.41 (m, 5H), 6.39 (s, 1H), 6.13 (s, 1H), $5.58(\mathrm{~s}, 1 \mathrm{H}), 0.88$ (s, 9H), 0.07 (s, 3H), -0.07 (s, $3 \mathrm{H}) ;{ }^{13} \mathrm{C}$ NMR $\left(62.5 \mathrm{MHz}, \mathrm{CDCl}_{3}\right.$ ): $\delta 169.8,142.9,141.9$, 128.1, 127.6, 126.8, 126.4, 72.8, 25.7, 18.2, -5.0; HRMS (ESI TOF) Calcd. for $\mathrm{C}_{16} \mathrm{H}_{24} \mathrm{O}_{3} \mathrm{Si}\left[\mathrm{M}+\mathrm{Na}^{+}\right.$]: 315.1393 . Found: 315.1338.

(土)-2-\{[(tert-Butyldimethylsilyl)oxy](4-methoxyphenyl) methyl\}prop-2-enoic acid (26): 98\% yield; viscous colorless oil; IR (film) $v_{\max } / \mathrm{cm}^{-1}: 2953,2925,2847,1691$, $1507,1245,1074,837,775 ;{ }^{1} \mathrm{H}$ NMR $\left(250 \mathrm{MHz}, \mathrm{CDCl}_{3}\right)$ : $\delta 7.25(\mathrm{~d}, 2 \mathrm{H}, J 7.0 \mathrm{~Hz}), 6.82(\mathrm{~d}, 2 \mathrm{H}, J 7.0 \mathrm{~Hz}), 6.35(\mathrm{~s}$, $1 \mathrm{H}), 6.11(\mathrm{~s}, 1 \mathrm{H}), 5.52(\mathrm{~s}, 1 \mathrm{H}), 3.79(\mathrm{~s}, 3 \mathrm{H}), 0.89(\mathrm{~s}, 9 \mathrm{H})$, 0.09 (s, 3H), -0.06 (s, 3H); ${ }^{13} \mathrm{C}$ NMR (125 MHz, $\left.\mathrm{CDCl}_{3}\right)$ : $\delta 170.1,158.9,143.1,134.2,128.1,126.0,113.5,72.4$, 55.2, 25.7, 18.1, -4.9, -5.1; HRMS (ESI TOF) Calcd. for $\mathrm{C}_{17} \mathrm{H}_{26} \mathrm{O}_{4} \mathrm{Si}\left[\mathrm{M}+\mathrm{Na}^{+}\right]$: 345.1493. Found: 345.1490.

( \pm )-2-\{[(tert-Butyldimethylsilyl)oxy](3,4,5-trimethoxyphenyl)methyl\}prop-2-enoic acid (27): 99\% yield; viscous oil; IR (film) $v_{\text {max }} / \mathrm{cm}^{-1}: 2955,2923,2849,1692,1509,1243$, 1077; ${ }^{1} \mathrm{H}$ NMR $\left(250 \mathrm{MHz}, \mathrm{CDCl}_{3}\right): \delta 6.58(\mathrm{~s}, 2 \mathrm{H}), 6.37$ (s, $1 \mathrm{H}), 6.08(\mathrm{~s}, 1 \mathrm{H}), 5.53(\mathrm{~s}, 1 \mathrm{H}), 3.81(\mathrm{~s}, 9 \mathrm{H}), 0.89(\mathrm{~s}, 9 \mathrm{H})$, $0.06(\mathrm{~s}, 3 \mathrm{H}),-0.05(\mathrm{~s}, 3 \mathrm{H}) ;{ }^{13} \mathrm{C}$ NMR $\left(62.5 \mathrm{MHz}, \mathrm{CDCl}_{3}\right)$ : $\delta 170.9,152.8,143.3,137.9,137.1,126.4,103.7,72.3$, $60.8,56.0,25.7,18.2,-4.9,-5.1$; HRMS (ESI TOF) Calcd. for $\mathrm{C}_{19} \mathrm{H}_{30} \mathrm{O}_{6} \mathrm{Si}\left[\mathrm{M}+\mathrm{Na}^{+}\right]$: 405.1709 . Found: 405.1566 .

( \pm )-2-\{[(tert-Butyldimethylsilyl)oxy](thiophen-3-yl) methyl\}prop-2-enoic acid (28): 99\% yield; viscous colorless oil; IR (film) $v_{\max } / \mathrm{cm}^{-1}: 2959,2931,2857,1691$, 1459, 1259, 1074, 837; ${ }^{1} \mathrm{H}$ NMR $\left(250 \mathrm{MHz}, \mathrm{CDCl}_{3}\right)$ : $\delta 7.20(\mathrm{dd}, 1 \mathrm{H}, J 5.0 / 1.3 \mathrm{~Hz}), 6.85-6.97(\mathrm{~m}, 2 \mathrm{H}), 6.45(\mathrm{t}$, $1 \mathrm{H}, J 1.0 \mathrm{~Hz}), 6.23$ (t, 1H, J $1.3 \mathrm{~Hz}), 5.87$ (s, 1H), 0.92 (s, 9H), 0.10 (s, 3H), 0.02 (s, 3H); ${ }^{13} \mathrm{C}$ NMR $(62.5 \mathrm{MHz}$, $\left.\mathrm{CDCl}_{3}\right): \delta 170.8,146.9,142.8,126.9,126.4,124.8,124.5$, 68.3, 25.7, 18.2, -5.1, -5.14; HRMS (ESI TOF) Calcd. for $\mathrm{C}_{14} \mathrm{H}_{22} \mathrm{O}_{3} \mathrm{SSi}\left[\mathrm{M}+\mathrm{Na}^{+}\right]$: 321.0957 . Found: 321.0883.

( \pm )-2-\{[(tert-Butyldimethylsilyl)oxy](2-fluorophenyl) methyl\}prop-2-enoic acid (29): 99\% yield; colorless oil; IR (film) $v_{\max } / \mathrm{cm}^{-1}: 2951,2928,2850,1692,1072,832,782$; ${ }^{1} \mathrm{H} \mathrm{NMR}\left(250 \mathrm{MHz}, \mathrm{CDCl}_{3}\right): \delta 10.44$ (s, $\left.1 \mathrm{H}, \mathrm{COO} \underline{\mathrm{H}}\right), 7.34$ (dt, $1 \mathrm{H}, J 1.8 / 7.5 \mathrm{~Hz}), 7.18-7.29(\mathrm{~m}, 1 \mathrm{H}), 6.95-7.15(\mathrm{~m}$, $2 \mathrm{H}), 6.47(\mathrm{~s}, 1 \mathrm{H}), 6.16(\mathrm{~s}, 1 \mathrm{H}), 5.92(\mathrm{~s}, 1 \mathrm{H}), 0.87(\mathrm{~s}, 9 \mathrm{H})$, 0.09 (s, 3H), $-0.08(\mathrm{~s}, 3 \mathrm{H}) ;{ }^{13} \mathrm{C}$ NMR (62.5 MHz, $\left.\mathrm{CDCl}_{3}\right)$ : $\delta 171.0,159.8(\mathrm{~d}, J 246.1 \mathrm{~Hz}), 142.1,129.3(\mathrm{~d}, J 3.0 \mathrm{~Hz})$, 129.1 (d, $J 2.1 \mathrm{~Hz}), 128.8$ (d, $J 3.8 \mathrm{~Hz}), 127.3,123.9$ (d, $J 3.5 \mathrm{~Hz}), 115.3$ (d, $J 22.0 \mathrm{~Hz}), 65.7,51.6,25.7,18.1$, -5.2, -5.24; HRMS (ESI TOF) Calcd. for $\mathrm{C}_{16} \mathrm{H}_{23} \mathrm{O}_{3} \mathrm{FSi}$ $\left[\mathrm{M}+\mathrm{Na}^{+}\right]:$333.1298. Found: 333.1207.

( \pm )-3-[(tert-Butyldimethylsilyl)oxy]-2-methylidenepentanoic acid (30): fluid oil; 99\% yield; IR (film) $\mathrm{v}_{\text {max }} / \mathrm{cm}^{-1}$ : 2923, 2854, 1691, 1253, 1084; ${ }^{1} \mathrm{H}$ NMR (250 MHz, $\left.\mathrm{CDCl}_{3}\right)$ : $\delta 11.60(\mathrm{~s}, 1 \mathrm{H}, \mathrm{COO} \underline{\mathrm{H}}), 6.41(\mathrm{~d}, 1 \mathrm{H}, J 1.2 \mathrm{~Hz}), 6.00(\mathrm{t}$, $1 \mathrm{H}, J 1.5 \mathrm{~Hz}), 4.55(\mathrm{t}, 1 \mathrm{H}, J 5.4 \mathrm{~Hz}), 1.41-1.80(\mathrm{~m}, 2 \mathrm{H})$, 0.78-1.00 (m, 12H), 0.08 (s, 3H), $0.01(\mathrm{~s}, 3 \mathrm{H}) ;{ }^{13} \mathrm{C}$ NMR $\left(62.5 \mathrm{MHz}, \mathrm{CDCl}_{3}\right): \delta 171.6,142.9,127.2,71.2,30.3,29.1$, 
25.7, 18.1, 9.1, -4.9, -5.1; HRMS (ESI TOF) Calcd. for $\mathrm{C}_{12} \mathrm{H}_{24} \mathrm{O}_{3} \mathrm{Si}\left[\mathrm{M}+\mathrm{H}^{+}\right]: 245.1573$. Found: 245.1554.

(土)-3-[(tert-Butyldimethylsilyl)oxy]-2-methylidenenonanoic acid (31): $98 \%$ yield; fluid oil; IR (film) $v_{\max } / \mathrm{cm}^{-1}$ : 2925, 2859, 1691, 1254, 1086, 833, 649; ${ }^{1} \mathrm{H}$ NMR $\left(250 \mathrm{MHz}, \mathrm{CDCl}_{3}\right): \delta 6.37(\mathrm{~s}, 1 \mathrm{H}), 5.92(\mathrm{~s}, 1 \mathrm{H}), 4.51-4.59$ (m, 1H), 1.20-1.40 (m, 10H), 0.82-0.93 (m, 12H), 0.08 (s, 3H), $0.02(\mathrm{~s}, 3 \mathrm{H}) ;{ }^{13} \mathrm{C}$ NMR $\left(62.5 \mathrm{MHz}, \mathrm{CDCl}_{3}\right), \delta$ : 170.6, 142.9, 126.9, 71.2, 37.7, 31.7, 29.1, 25.7, 25.0, 22.6, 18.1, 14.1, -5.0; HRMS (ESI TOF) Calcd. for $\mathrm{C}_{16} \mathrm{H}_{32} \mathrm{O}_{3} \mathrm{Si}$ $\left[\mathrm{M}+\mathrm{H}^{+}\right]$: 301.2199. Found: 301.2232.

(士)-3-[(tert-Butyldimethylsilyl)oxy]-2-methyleneoctadecanoic acid (32): 99\% yield; white solid; mp $45-47^{\circ} \mathrm{C}$; IR (film) $v_{\max } / \mathrm{cm}^{-1}: 2918,2851,1681,1623,1472$, 1090,$838 ;{ }^{1} \mathrm{HNMR}\left(250 \mathrm{MHz}, \mathrm{CDCl}_{3}\right): \delta 6.34-6.36(\mathrm{~m}, 1 \mathrm{H})$, 5.86-5.88 (m, 1H), $4.53(\mathrm{t}, 1 \mathrm{H}, J 5.6 \mathrm{~Hz}), 1.45-1.63(\mathrm{~m}, 2 \mathrm{H})$, $1.18-1.39(\mathrm{~m}, 26 \mathrm{H}), 0.83-0.93(\mathrm{~m}, 12 \mathrm{H}), 0.1(\mathrm{~s}, 3 \mathrm{H}), 0.03(\mathrm{~s}$, $3 \mathrm{H}) ;{ }^{13} \mathrm{C} \mathrm{NMR}\left(62.5 \mathrm{MHz}, \mathrm{CDCl}_{3}\right): \delta 169.7,142.5,126.8$, $71.8,37.6,31.9,29.7,29.64,29.6,29.5,29.5,29.4,29.3$, 25.8, 25.1, 22.7, 18.1, 14.1, -4.8, -5.0; HRMS (ESI TOF) Calcd. for $\mathrm{C}_{25} \mathrm{H}_{50} \mathrm{O}_{3} \mathrm{Si}[\mathrm{M}+\mathrm{H}]^{+}$: 427.3607. Found: 427.3599 .

\section{General procedure for the preparation of $\alpha$-hydroxyketones} (acyloins)

To a stirred $0.2 \mathrm{~mol} \mathrm{~L}^{-1}$ solution of carboxylic acid in acetone at $0{ }^{\circ} \mathrm{C}$ was added triethylamine (2 equiv.) and ethyl chloroformate (1.5 equiv.). The mixture was stirred at $0{ }^{\circ} \mathrm{C}$ and carbonate formation was observed by TLC after $5 \mathrm{~min}$. After that, $\mathrm{NaN}_{3}$ was added ( 2.5 equiv.). The resulting mixture was vigorously stirred for $2 \mathrm{~h}$ until the formation of a slightly apolar layer (acylazide formation). Then, the crude mixture was diluted in cold dichloromethane and washed with cold water. The organic layer was dried over anhydrous $\mathrm{Na}_{2} \mathrm{SO}_{4}$ and the solvent was removed under vacuum. To this crude material, under an argon atmosphere, dry toluene $\left(0.1 \mathrm{~mol} \mathrm{~L}^{-1}\right)$ was added and the mixture was refluxed for $2 \mathrm{~h}$. The solvent was then removed under reduced pressure and the resulting product was diluted in water. The mixture was refluxed for $12 \mathrm{~h}$. Then, the reaction mixture was coolled to room temperature and extracted with ethyl acetate. The organic layer was dried over anhydrous $\mathrm{Na}_{2} \mathrm{SO}_{4}$ and the solvent was removed under vacuum. The residue was purified by flash silica gel column chromatography (ethyl acetate:hexanes, up to 25:75) to provide the required acyloins. Yields refer to 3 steps from MBH adducts.

(土)-1-[(tert-Butyldimethylsilyl)oxy]-1-(2-nitrophenyl) propan-2-one (33): $46 \%$ yield; pale yellow oil; IR (film) $v_{\max } / \mathrm{cm}^{-1}: 2953,2933,2851,1728,1527,1343,1254$, 1102,$833 ;{ }^{1} \mathrm{H}$ NMR $\left(250 \mathrm{MHz}, \mathrm{CDCl}_{3}\right): \delta 7.97-8.03(\mathrm{~m}$, $1 \mathrm{H})$, 7.58-7.77 (m, 2H), 7.42-7.51 (m, 1H), $5.72(\mathrm{~s}, 1 \mathrm{H})$, $2.31(\mathrm{~s}, 3 \mathrm{H}), 0.91(\mathrm{~s}, 9 \mathrm{H}), 0.16(\mathrm{~s}, 3 \mathrm{H}),-0.07(\mathrm{~s}, 3 \mathrm{H})$; ${ }^{13} \mathrm{C}$ NMR $\left(62.5 \mathrm{MHz}, \mathrm{CDCl}_{3}\right): \delta 205.8,147.7,135.0$, 133.2, 129.2, 128.8, 124.8, 26.3, 25.6, 18.1, -5.0; HRMS (ESI TOF) Calcd. for $\mathrm{C}_{15} \mathrm{H}_{23} \mathrm{O}_{4} \mathrm{NSi}\left[\mathrm{M}+\mathrm{H}^{+}\right]$: 310.1475 . Found: 310.1600.

( \pm )-1-[(tert-Butyldimethylsilyl)oxy]-1-(3-chlorophenyl) propan-2-one (34): $45 \%$ yield; colorless oil; IR (film) $v_{\max } / \mathrm{cm}^{-1}: 2953,2929,2864,1719,1470,1258,1111,775$; ${ }^{1} \mathrm{H}$ NMR $\left(250 \mathrm{MHz}, \mathrm{CDCl}_{3}\right): \delta 7.43(\mathrm{~s}, 1 \mathrm{H}), 7.23-7.33$ $(\mathrm{m}, 3 \mathrm{H}), 5.00(\mathrm{~s}, 1 \mathrm{H}), 2.12(\mathrm{~s}, 3 \mathrm{H}), 0.96(\mathrm{~s}, 9 \mathrm{H}), 0.09(\mathrm{~s}$, $3 \mathrm{H}), 0.02(\mathrm{~s}, 3 \mathrm{H}) ;{ }^{13} \mathrm{C} \mathrm{NMR}\left(62.5 \mathrm{MHz}, \mathrm{CDCl}_{3}\right): \delta 208.5$, 140.7, 134.5, 129.8, 128.2, 125.9, 123.9, 80.6, 25.7, 23.9, 18.2, -4.9; HRMS (ESI TOF) Calcd. for $\mathrm{C}_{15} \mathrm{H}_{23} \mathrm{O}_{2} \mathrm{ClSi}$ $\left[\mathrm{M}+\mathrm{Na}^{+}\right]: 321.1053$. Found: 321.1056

(土)-1-[(tert-Butyldimethylsilyl)oxy]-1-phenylpropan2-one (35): 50\% yield; pale yellow oil; IR (film) $v_{\max } / \mathrm{cm}^{-1}$ : 2949, 2929, 2855, 1711, 1258, 1102, 865, 837; ' ${ }^{1} \mathrm{H}$ NMR $\left(250 \mathrm{MHz}, \mathrm{CDCl}_{3}\right): \delta$ 7.25-7.45 (m, 5H), $5.04(\mathrm{~s}, 1 \mathrm{H})$, $2.11(\mathrm{~s}, 3 \mathrm{H}), 0.96$ (s, 9H), $0.10(\mathrm{~s}, 3 \mathrm{H}), 0.01$ (s, 3H); ${ }^{13} \mathrm{C} \mathrm{NMR}\left(125 \mathrm{MHz}, \mathrm{CDCl}_{3}\right): \delta 209.1,199.5,138.6,128.5$, 128.0, 125.8, 81.2, 25.7, 23.9, 18.2, -4.9, -5.2; HRMS (ESI TOF) Calcd. for $\mathrm{C}_{15} \mathrm{H}_{24} \mathrm{O}_{2} \mathrm{Si}\left[\mathrm{M}+\mathrm{H}^{+}\right]$: 265.1624 . Found: 265.1685.

(士)-1-[(tert-Butyldimethylsilyl)oxy]-1-(4-methoxyphenyl)propan-2-one (36): $48 \%$ yield; pale yellow oil; IR (film) $v_{\max } / \mathrm{cm}^{-1}: 2949,2855,1715,1511,1090,841$, 784; ${ }^{1} \mathrm{H}$ NMR $\left(250 \mathrm{MHz}, \mathrm{CDCl}_{3}\right): \delta 7.30(\mathrm{~d}, 2 \mathrm{H}, J 6.7 \mathrm{~Hz})$, 6.87 (d, 2H, J 6.7 Hz), 4.99 (s, 1H), 3.80 (s, 3H), 2.11 (s, $3 \mathrm{H}), 0.94$ (s, 9H), 0.08 (s, 3H), -0.01 (s, 3H); ${ }^{13} \mathrm{C}$ NMR $\left(62.5 \mathrm{MHz}, \mathrm{CDCl}_{3}\right): \delta 209.2,159.4,130.7,127.1,113.9$, 80.8, 55.2, 25.7, 23.8, 18.2, -5.0; HRMS (ESI TOF) Calcd. for $\mathrm{C}_{16} \mathrm{H}_{26} \mathrm{O}_{3} \mathrm{Si}\left[\mathrm{M}+\mathrm{Na}^{+}\right]$: 317.1549. Found: 317.1505.

(士)-1-[(tert-Butyldimethylsilyl)oxy]-1-(3,4,5trimethoxyphenyl)propan-2-one (37): $42 \%$ yield; colorless oil; IR (film) $v_{\max } / \mathrm{cm}^{-1}: 2947,2853,1714,1512,1089,843$; ${ }^{1} \mathrm{H}$ NMR $\left(250 \mathrm{MHz}, \mathrm{CDCl}_{3}\right): \delta 6.65(\mathrm{~s}, 2 \mathrm{H}), 4.95(\mathrm{~s}, 1 \mathrm{H})$, 3.75-3.88 (m, 9H), $2.10(\mathrm{~s}, 3 \mathrm{H}), 0.95(\mathrm{~s}, 9 \mathrm{H}), 0.07(\mathrm{~s}, 3 \mathrm{H})$, 0.03 (s, 3H); ${ }^{13} \mathrm{C} \mathrm{NMR}\left(62.5 \mathrm{MHz}, \mathrm{CDCl}_{3}\right): \delta 208.8,153.3$, 137.5, 134.0, 102.4, 80.9, 60.8, 56.0, 25.7, 23.7, 18.2, -4.9, -5.1 ; HRMS (ESI TOF) Calcd. for $\mathrm{C}_{18} \mathrm{H}_{30} \mathrm{O}_{5} \mathrm{Si}\left[\mathrm{M}+\mathrm{Na}^{+}\right]$: 377.1760. Found: 377.1657.

( \pm )-1-[(tert-Butyldimethylsilyl)oxy]-1-(thiophen-2-yl) propan-2-one (38): $44 \%$ yield; pale yellow oil; IR (film) 
$\mathrm{V}_{\max } / \mathrm{cm}^{-1}: 2949,2851,1712,1091 ;{ }^{1} \mathrm{H}$ NMR $(250 \mathrm{MHz}$, $\left.\mathrm{CDCl}_{3}\right): \delta$ 7.22-7.28 (m, $\left.1 \mathrm{H}\right), 6.96-7.01(\mathrm{~m}, 2 \mathrm{H}), 5.24(\mathrm{~s}$, $1 \mathrm{H}), 2.20(\mathrm{~s}, 3 \mathrm{H}), 0.95$ (s, 9H), $0.10(\mathrm{~s}, 3 \mathrm{H}), 0.05(\mathrm{~s}, 3 \mathrm{H})$; ${ }^{13} \mathrm{C} \mathrm{NMR}\left(62.5 \mathrm{MHz}, \mathrm{CDCl}_{3}\right): \delta 207.7,153.3,142.6,127.1$, 125.2, 124.3, 77.8, 25.6, 23.7, 18.2, -5.1, -5.2; HRMS (ESI TOF) Calcd. for $\mathrm{C}_{13} \mathrm{H}_{22} \mathrm{O}_{2} \mathrm{SSi}\left[\mathrm{M}+\mathrm{Na}^{+}\right]$: 293.1007. Found: 293.0922.

( \pm )-1-[(tert-Butyldimethylsilyl)oxy]-1-(2-fluorophenyl) propan-2-one (39): $41 \%$ yield; colorless oil; IR (film) $v_{\max } / \mathrm{cm}^{-1}: 2951,1720,1471,1256,1109 ;{ }^{1} \mathrm{H}$ NMR $\left(250 \mathrm{MHz}, \mathrm{CDCl}_{3}\right): \delta 7.47(\mathrm{dt}, 1 \mathrm{H}, J 1.8 / 7.5 \mathrm{~Hz}), 7.23-7.35$ (m, 1H), 7.15 (dt, 1H, J 1.2/7.5 Hz), 7.00-7.09 (m, 1H), $5.34(\mathrm{~s}, 1 \mathrm{H}), 2.18(\mathrm{~s}, 3 \mathrm{H}), 0.92(\mathrm{~s}, 9 \mathrm{H}), 0.11(\mathrm{~s}, 3 \mathrm{H}),-0.05$ (s, 3H); $\left.{ }^{13} \mathrm{C} \mathrm{NMR} \mathrm{(62.5} \mathrm{MHz,} \mathrm{CDCl}_{3}\right): \delta 207.1,159.8(\mathrm{~d}$, $J 245.4 \mathrm{~Hz}), 129.8$ (d, J 8.1 Hz), $128.2(\mathrm{~d}, J 3.8 \mathrm{~Hz}), 126.6$ (d, $J 14.3 \mathrm{~Hz}), 124.3$ (d, $J 3.5 \mathrm{~Hz}), 115.4(\mathrm{~d}, J 21.5 \mathrm{~Hz})$, 74.9, 25.6, 24.9, 18.1, -5.1, -5.2; HRMS (ESI TOF) Calcd. for $\mathrm{C}_{15} \mathrm{H}_{23} \mathrm{O}_{2} \mathrm{FSi}\left[\mathrm{M}+\mathrm{Na}^{+}\right]$: 305.1349. Found: 305.1284 .

(士)-3-[(tert-Butyldimethylsilyl)oxy]pentan-2-one (40): $46 \%$ yield; colorless oil; ${ }^{1} \mathrm{H}$ NMR $\left(250 \mathrm{MHz}, \mathrm{CDCl}_{3}\right)$ : $\delta 3.90$ (dd, 1H, J 5.6/6.5 Hz), 2.13 (s, 3H), 1.50-1.71 (m, 2H), 0.77-0.93 (m, 12H), 0.04 (s, 3H), 0.03 (s, 3H); ${ }^{13} \mathrm{C}$ NMR $\left(62.5 \mathrm{MHz}, \mathrm{CDCl}_{3}\right): \delta 212.5,79.9,27.8,25.3$, 18.0, 9.1, -5.0, -5.1; HRMS (ESI TOF) Calcd. for $\mathrm{C}_{11} \mathrm{H}_{24} \mathrm{O}_{2} \mathrm{Si}\left[\mathrm{M}+\mathrm{H}^{+}\right]$: 217.1624. Found: 217.1608.

( \pm )-3-[(tert-Butyldimethylsilyl)oxy]nonan-2-one (41): $42 \%$ yield; pale yellow oil; ${ }^{1} \mathrm{H}$ NMR $\left(250 \mathrm{MHz}, \mathrm{CDCl}_{3}\right)$ : d 3.92-4.03 (m, 1H), $2.15(\mathrm{~s}, 3 \mathrm{H}), 1.18-1.45(\mathrm{~m}, 10 \mathrm{H}), 0.82-$ $1.09(\mathrm{~m}, 12 \mathrm{H}), 0.05(\mathrm{~s}, 6 \mathrm{H}) ;{ }^{13} \mathrm{C} \mathrm{NMR}\left(62.5 \mathrm{MHz}, \mathrm{CDCl}_{3}\right)$ : $\delta$ 212.8, 79.0, 34.8, 31.7, 29.2, 25.7, 25.2, 24.7, 22.6, 18.1, 14.1, -4.9; HRMS (ESI TOF) Calcd. for $\mathrm{C}_{15} \mathrm{H}_{32} \mathrm{O}_{2} \mathrm{Si}[\mathrm{M}+$ $\mathrm{Na}^{+}$]: 295.4887. Found: 295.4849.

( \pm )-3-[(tert-Butyldimethylsilyl)oxy] octadecan-2-one (42): $40 \%$ yield; colorless viscous oil; IR (film) $v_{\max } / \mathrm{cm}^{-1}$ : 2926, 2855, 1719, 1465, 1253, 1104; ${ }^{1} \mathrm{H}$ NMR $(250 \mathrm{MHz}$, $\mathrm{CDCl}_{3}$ ): $\delta 3.97(\mathrm{dd}, 1 \mathrm{H}, J 5.3 / 6.9 \mathrm{~Hz}), 2.15$ (s, 3H), 1.46$1.55(\mathrm{~m}, 2 \mathrm{H}), 1.17-1.40(\mathrm{~m}, 26 \mathrm{H}), 0.83-0.97(\mathrm{~m}, 12 \mathrm{H}), 0.06$ (s, 3H), 0.05 (s, 3H); ${ }^{13} \mathrm{C} \mathrm{NMR} \mathrm{(62.5} \mathrm{MHz,} \mathrm{CDCl}_{3}$ ): $\delta 212.7$, 79.0, 34.8, 31.9, 29.7, 29.64, 29.6, 29.5, 29.4, 29.3, 25.7, 25.2, 24.7, 22.7, 18.1, 14.1, -5.0; HRMS (ESI TOF) Calcd. for $\mathrm{C}_{24} \mathrm{H}_{50} \mathrm{O}_{2} \mathrm{Si}\left[\mathrm{M}+\mathrm{Na}^{+}\right]$: 421.3478. Found: 421.3453 .

General procedure for the diastereoselective preparation of vicinal alcohols

To a $0.2 \mathrm{~mol} \mathrm{~L}^{-1}$ methanolic solution of acyloins was added an amine ( 3 equiv.). The resulting solution was vigorously stirred at room temperature for $1 \mathrm{~h}$. After, the solution was cooled to $-78{ }^{\circ} \mathrm{C}$ and $\mathrm{LiBH}_{4}$ (1.2 equiv.) was added. The mixture was then warmed to room temperature and stirred for $12 \mathrm{~h}$. Then, the medium was diluted with ethyl acetate $(15 \mathrm{~mL})$ and the organic phase was washed with water $(5 \times 7 \mathrm{~mL})$, brine $(10 \mathrm{~mL})$, dried over anhydrous $\mathrm{Na}_{2} \mathrm{SO}_{4}$ and the solvent was removed under vacuum. The residue was purified through silica gel (solvent: hexanes:ethyl acetate, up to 65:35) to provide the corresponding vicinal aminoalcohols, in good yields and in high diastereoselectivities.

( \pm )-1,2-anti-1-[(tert-Butyldimethylsilyl)oxy]-1(3-chlorophenyl)propan-2-yl](2-phenylethyl)amine (43): $79 \%$ yield; yellowish oil; IR (film) $v_{\max } / \mathrm{cm}^{-1}: 2955,2929$, 2857, 1471, 1254, 1075, 836; ${ }^{1} \mathrm{H} \mathrm{NMR}\left(250 \mathrm{MHz}, \mathrm{CDCl}_{3}\right)$ : $\delta$ 7.08-7.32 (m, 9H), 4.55 (d, 1H, J 4.7 Hz), 2.62-2.99 (m, $5 \mathrm{H}), 0.98(\mathrm{~d}, 3 \mathrm{H}, J 6.4 \mathrm{~Hz}), 0.86(\mathrm{~s}, 9 \mathrm{H}),-0.003(\mathrm{~s}, 3 \mathrm{H})$, -0.20 (s, 3H); ${ }^{13} \mathrm{CNMR}\left(62.5 \mathrm{MHz}, \mathrm{CDCl}_{3}\right)$ : $\delta 145.1,139.9$, 133.9, 129.2, 128.6, 128.4, 127.3, 126.8, 126.1, 124.8, 77.0, 59.7, 48.6, 36.5, 25.8, 18.1, 14.9, -4.6, -5.1; HRMS (ESI TOF) Calcd. for $\mathrm{C}_{23} \mathrm{H}_{34}$ CINOSi [M + $\mathrm{H}^{+}$]: 404.2177. Found: 404.1996.

(士)-1,2-anti-Benzyl-[1-[(tert-butyldimethylsilyl)oxy]1-(3-chlorophenyl)propan-2-yl]amine (44): 77\% yield; yellowish oil. IR (film) $v_{\max } / \mathrm{cm}^{-1}: 2929,2857,1471,1255$, 1075,836 ; ${ }^{1} \mathrm{H}$ NMR $\left(250 \mathrm{MHz}, \mathrm{CDCl}_{3}\right): \delta 7.12-7.37$ (m, 9H), 4.63 (d, 1H, J 4.8 Hz), 3.82 (d, 1H, J $13.5 \mathrm{~Hz}), 3.71$ (d, 1H, J 13.5 Hz), $2.77(\mathrm{dq}, 1 \mathrm{H}, J 4.8 / 6.4 \mathrm{~Hz}), 1.02(\mathrm{~d}$, 3H, J $6.4 \mathrm{~Hz}$ ), 0.89 (s, 9H), 0.05 (s, 3H), -0.16 (s, 3H); ${ }^{13} \mathrm{C} \mathrm{NMR}\left(62.5 \mathrm{MHz}, \mathrm{CDCl}_{3}\right): \delta 145.2,140.4,133.9,129.2$, 128.3, 127.8, 127.3, 127.0, 126.8, 125.0, 77.1, 58.6, 51.0, 25.8, 18.1, 15.1, -4.6, -4.9; HRMS (ESI TOF) Calcd. for $\mathrm{C}_{22} \mathrm{H}_{32} \mathrm{ONClSi}\left[\mathrm{M}+\mathrm{H}^{+}\right]$: 390.2020. Found: 370.1797 .

( \pm )-1,2-anti-\{1-[(tert-Butyldimethylsilyl)oxy]1-phenylpropan-2-yl\}(2-phenylethyl)amine (45): 85\% yield; yellowish oil; IR (film) $v_{\max } / \mathrm{cm}^{-1}: 2928,2857,1453$, $1255,1063,836$; ${ }^{1} \mathrm{H}$ NMR $\left(250 \mathrm{MHz}, \mathrm{CDCl}_{3}\right): \delta$ 7.09-7.32 (m, 10H), $4.58(\mathrm{~d}, 1 \mathrm{H}, J 5.0 \mathrm{~Hz}), 2.61-2.99(\mathrm{~m}, 5 \mathrm{H}), 1.02$ (d, 3H, J 6.4 Hz), 0.86 (s, 9H), -0.001 (s, 3H), -0.22 (s, $3 \mathrm{H}) ;{ }^{13} \mathrm{C}$ NMR (62.5 MHz, $\mathrm{CDCl}_{3}$ ): $\delta 142.9,140.0,128.6$, 128.4, 127.9, 127.1, 126.7, 126.0, 77.6, 59.8, 48.7, 36.6, 25.8, 18.1, 15.1, -4.6, -5.1; HRMS (ESI TOF) Calcd. for $\mathrm{C}_{23} \mathrm{H}_{35} \mathrm{ONSi}\left[\mathrm{M}+\mathrm{H}^{+}\right]$: 370.2566. Found: 370.2747 .

(士)-1,2-anti-Benzyl(\{1-[(tert-butyldimethylsilyl)oxy]1-phenylpropan-2-yl\})amine (46): 83\% yield; yellowish oil; IR (film) $v_{\max } / \mathrm{cm}^{-1}: 2956,2929,2857,1493,1254,1063$, 1028,$836 ;{ }^{1} \mathrm{H}$ NMR $\left(250 \mathrm{MHz}, \mathrm{CDCl}_{3}\right): \delta 7.12-7.38(\mathrm{~m}$, 
$10 \mathrm{H}), 4.64(\mathrm{~d}, 1 \mathrm{H}, J 5.1 \mathrm{~Hz}), 3.81(\mathrm{~d}, 1 \mathrm{H}, J 13.5 \mathrm{~Hz}), 3.72$ $(\mathrm{d}, 1 \mathrm{H}, J 13.5 \mathrm{~Hz}), 2.80(\mathrm{dq}, 1 \mathrm{H}, J 5.1 / 6.4 \mathrm{~Hz}), 1.05(\mathrm{~d}, 3 \mathrm{H}$, $J 6.4 \mathrm{~Hz}), 0.90$ (s, 9H), 0.05 (s, 3H), $-0.18(\mathrm{~s}, 3 \mathrm{H}) ;{ }^{13} \mathrm{C} \mathrm{NMR}$ $\left(62.5 \mathrm{MHz}, \mathrm{CDCl}_{3}\right): \delta 142.9,140.6,128.3,127.9,127.8$, 127.1, 126.9, 126.7, 77.8, 58.7, 51.0, 25.8, 18.2, 15.4, -4.5, -5.0 ; HRMS (ESI TOF) Calcd. for $\mathrm{C}_{22} \mathrm{H}_{33} \mathrm{ONSi}\left[\mathrm{M}+\mathrm{H}^{+}\right]$: 356.2410. Found: 356.2561.

(士)-1,2-anti-\{1-[(tert-Butyldimethylsilyl)oxy]1-phenylpropan-2-yl\}(prop-2-en-1-yl)amine (47): 82\% yield; yellowish oil; IR (film) $v_{\max } / \mathrm{cm}^{-1}: 2956,2929,2857$, $1471,1255,1063,867,837 ;{ }^{1} \mathrm{H}$ NMR $\left(250 \mathrm{MHz}, \mathrm{CDCl}_{3}\right)$ : $\delta$ 7.17-7.42 (m, 5H), 5.73-5.95 (m, 1H), 5.00-5.21 (m, 2H), $4.65(\mathrm{~d}, 1 \mathrm{H}, J 4.8 \mathrm{~Hz}), 3.12-3.39(\mathrm{~m}, 2 \mathrm{H}), 2.80(\mathrm{dq}, 1 \mathrm{H}$, $J 4.8 / 6.4 \mathrm{~Hz}$ ), 0.99 (d, 3H, J 6.4 Hz), 0.90 (s, 9H), 0.05 (s, $3 \mathrm{H}),-0.19(\mathrm{~s}, 3 \mathrm{H}) ;{ }^{13} \mathrm{C} \mathrm{NMR}\left(62.5 \mathrm{MHz}, \mathrm{CDCl}_{3}\right): \delta 142.9$, 137.0, 128.3, 127.9, 127.1, 126.7, 115.4, 77.3, 58.9, 49.6, 25.8, 18.2, 14.9, -4.5, -5.0; HRMS (ESI TOF) Calcd. for $\mathrm{C}_{18} \mathrm{H}_{31} \mathrm{ONSi}\left[\mathrm{M}+\mathrm{H}^{+}\right]$: 306.2253. Found: 306.2394 .

(士)-1,2-anti-N-\{1-[(tert-Butyldimethylsilyl)oxy]1-phenylpropan-2-yl)cyclohexanamine (48): $80 \%$ yield; yellowish oil; IR (film) $v_{\max } / \mathrm{cm}^{-1}: 2928,2855,1451,1255$, $1066,862,836 ;{ }^{1} \mathrm{H}$ NMR $\left(250 \mathrm{MHz}, \mathrm{CDCl}_{3}\right): \delta 7.15-7.40$ $(\mathrm{m}, 5 \mathrm{H}), 4.61(\mathrm{~d}, 1 \mathrm{H}, J 4.7 \mathrm{~Hz}), 2.89(\mathrm{dq}, 1 \mathrm{H}, J 4.7 / 6.3 \mathrm{~Hz})$, 2.41-2.60 (m, 1H), 1.50-1.92 (m, 6H), 1.09-1.35 (m, 4H), 0.97 (d, 3H, J 6.3 Hz), 0.90 (s, 9H), 0.04 (s, 3H), -0.20 (s, $3 \mathrm{H}) ;{ }^{13} \mathrm{C} \mathrm{NMR}\left(62.5 \mathrm{MHz}, \mathrm{CDCl}_{3}\right): \delta 143.3,127.8,127.0$, 77.5, 55.8, 52.9, 34.1, 33.1, 26.2, 25.6, 25.0, 24.9, 18.2, 15.5, $-4.5,-5.0$; HRMS (ESI TOF) Calcd. for $\mathrm{C}_{21} \mathrm{H}_{37} \mathrm{ONSi}$ $\left[\mathrm{M}+\mathrm{H}^{+}\right]$: 348.2723. Found: 348.2834.

( \pm )-1,2-anti-\{1-[(tert-Butyldimethylsilyl)oxy]-1(3,4,5-trimethoxyphenyl)propan-2-yl)(2-phenylethyl) amine (49): 81\% yield; colorless oil; IR (film) $v_{\max }$ I $\mathrm{cm}^{-1}: 2933,1592,1455,1321,1128,836,776 ;{ }^{1} \mathrm{H}$ NMR $\left(250 \mathrm{MHz}, \mathrm{CDCl}_{3}\right): \delta$ 7.05-7.27 $(\mathrm{m}, 5 \mathrm{H})$, $6.48(\mathrm{~s}, 2 \mathrm{H}), 4.45(\mathrm{~d}, 1 \mathrm{H}, J 5.4 \mathrm{~Hz}), 3.84(\mathrm{~s}, 3 \mathrm{H})$, $3.81(\mathrm{~s}, 6 \mathrm{H}), 2.62-3.00(\mathrm{~m}, 5 \mathrm{H}), 1.05(\mathrm{~d}, 3 \mathrm{H}$, $J 6.1 \mathrm{~Hz}), 0.86(\mathrm{~s}, 9 \mathrm{H}), 0.01$ (s, 3H), -0.19 (s, 3H); ${ }^{13} \mathrm{C}$ NMR $\left(62.5 \mathrm{MHz}, \mathrm{CDCl}_{3}\right): \delta 152.8,139.8,138.6$, $136.8,128.5,128.4,126.1,103.4,77.9,60.9,59.9,56.0$, 48.6, 36.4, 25.8, 18.1, 15.3, -4.5, -5.1; HRMS (ESI TOF) Calcd. for $\mathrm{C}_{26} \mathrm{H}_{41} \mathrm{O}_{4} \mathrm{NSi}\left[\mathrm{M}+\mathrm{H}^{+}\right]$: 460.2805. Found: 460.2871 .

(士)-1,2-anti-\{1-[(tert-Butyldimethylsilyl)oxy]-1-(3,4,5trimethoxyphenyl)propan-2-yl\}(prop-2-en-1-yl)amine (50): $80 \%$ yield; colorless oil; IR (film) $v_{\max } / \mathrm{cm}^{-1}: 2930,2857$, $1592,1420,1330,1130,869,837$; ${ }^{1} \mathrm{H}$ NMR $(250 \mathrm{MHz}$, $\left.\mathrm{CDCl}_{3}\right): \delta 6.54(\mathrm{~s}, 2 \mathrm{H}), 5.75-5.92(\mathrm{~m}, 1 \mathrm{H}), 5.01-5.17(\mathrm{~m}$,
$2 \mathrm{H}), 4.55(\mathrm{~d}, 1 \mathrm{H}, \mathrm{J} 5.0 \mathrm{~Hz}), 3.84(\mathrm{~s}, 9 \mathrm{H}), 3.10-3.37$ (m, 2H), 2.80 (dq, 1H, J 5.0/6.3 Hz), 1.03 (d, 3H, J $6.3 \mathrm{~Hz}), 0.91$ (s, 9H), 0.06 (s, 3H), -0.14 (s, 3H); ${ }^{13} \mathrm{C}$ NMR $(62.5 \mathrm{MHz}$, $\left.\mathrm{CDCl}_{3}\right): \delta 152.8,138.7,136.9,136.8,115.4,103.4,77.6$, 60.9, 59.0, 56.0, 49.6, 25.8, 18.2, 15.1, -4.5, -5.0; HRMS (ESI TOF) Calcd. for $\mathrm{C}_{21} \mathrm{H}_{37} \mathrm{O}_{4} \mathrm{NSi}\left[\mathrm{M}+\mathrm{H}^{+}\right.$]: 396.2570. Found: 396.2693.

(士)-1,2-anti-\{1-[(tert-Butyldimethylsilyl)oxy]1-(thiophen-2-yl)propan-2-yl)(2-phenylethyl)amine (51): $82 \%$ yield; yellowish oil; IR (film) $v_{\max } / \mathrm{cm}^{-1}: 2955,2929$, 2857, 1471, 1253, 1075, 836; ${ }^{1} \mathrm{H} \mathrm{NMR}\left(250 \mathrm{MHz}, \mathrm{CDCl}_{3}\right)$ : $\delta$ 7.09-7.27 (m, 6H), 6.80-6.93 (m, 2H), $4.76(\mathrm{~d}, J 5.6 \mathrm{~Hz}$, $1 \mathrm{H}), 2.63-2.99(\mathrm{~m}, 5 \mathrm{H}), 1.08(\mathrm{~d}, 3 \mathrm{H}, J 6.3 \mathrm{~Hz}), 0.85(\mathrm{~s}, 9 \mathrm{H})$, $0.02(\mathrm{~s}, 3 \mathrm{H}),-0.15(\mathrm{~s}, 3 \mathrm{H}) ;{ }^{13} \mathrm{C}$ NMR $\left(62.5 \mathrm{MHz}, \mathrm{CDCl}_{3}\right)$ : $\delta 146.9,140.0,128.6,128.4,126.002,126.0,124.5,124.3$, 74.7, 60.1, 48.7, 36.5, 25.7, 18.1, 15.9, -4.6, -5.1; HRMS (ESI TOF) Calcd. for $\mathrm{C}_{21} \mathrm{H}_{33} \mathrm{ONSSi}\left[\mathrm{M}+\mathrm{H}^{+}\right]$: 376.2130. Found: 376.2141.

(士)-1,2-anti-\{1-[(tert-Butyldimethylsilyl)oxy]-1-(2fluorophenyl)propan-2-yl\}(2-phenylethyl)amine (52): 78\% yield; yellowish oil; IR (film) $v_{\max } / \mathrm{cm}^{-1}: 2857,1486,1254$, 1033, 837; ${ }^{1} \mathrm{H}$ NMR $\left(250 \mathrm{MHz}, \mathrm{CDCl}_{3}\right): \delta 7.43(\mathrm{dt}, 1 \mathrm{H}$, $\left.J_{1} 1.8, J_{2} 7.5 \mathrm{~Hz}\right), 7.05-7.35(\mathrm{~m}, 8 \mathrm{H}), 6.90-7.01(\mathrm{~m}, 1 \mathrm{H})$, $5.07(\mathrm{~d}, 1 \mathrm{H}, J 3.9 \mathrm{~Hz}), 2.62-2.99(\mathrm{~m}, 5 \mathrm{H}), 0.97(\mathrm{~d}, 3 \mathrm{H}$, $J 6.5 \mathrm{~Hz}), 0.87$ (s, 9H), 0.02 (s, 3H), 0.01 (s, 3H); ${ }^{13} \mathrm{C}$ NMR $\left(62.5 \mathrm{MHz}, \mathrm{CDCl}_{3}\right): \delta 159.4(\mathrm{~d}, J 242.8 \mathrm{~Hz}), 140.2,130.0$ (d, $J 13.3 \mathrm{~Hz}$ ), 128.8 (m, C-Ar), 128.4 (m, C-Ar), 126.0, 123.7 (d, J 3.4 Hz), 114.8 (d, $J 22.0 \mathrm{~Hz}$ ), 70.0, 58.3, 48.7, $36.7,25.8,18.1,14.8,-4.8,-5.2$; HRMS (ESI TOF) Calcd. for $\mathrm{C}_{23} \mathrm{H}_{34} \mathrm{ONFSi}\left[\mathrm{M}+\mathrm{H}^{+}\right]$: 388.2472. Found: 388.2512.

( \pm )-1,2-anti-\{3-[(tert-Butyldimethylsilyl)oxy]pentan2-yl\}(2-phenylethyl)amine (53): 73\% yield; yellowish oil; IR (film) $v_{\text {max }} / \mathrm{cm}^{-1}: 2958,2930,2857,1470,1254,1007$, 835; ${ }^{1} \mathrm{H}$ NMR $\left(250 \mathrm{MHz}, \mathrm{CDCl}_{3}\right): \delta$ 7.12-7.37 (m, 5H), 3.49-3.60 (m, 1H), 2.60-2.99 (m, 5H), 1.38-1.52 (m, 2H), 0.98 (d, 3H, J 6.5 Hz), 0.78-0.90 (m, 12H), 0.03 (s, 3H), -0.01 (s, 3H); ${ }^{13} \mathrm{C} \mathrm{RMN}\left(62.5 \mathrm{MHz}, \mathrm{CDCl}_{3}\right): \delta 140.2,128.7$, 128.4, 126.1, 76.6, 56.9, 49.3, 36.9, 25.8, 25.3, 18.0, 15.0, $10.4,-4.4,-4.5$; HRMS (ESI TOF) Calcd. for $\mathrm{C}_{19} \mathrm{H}_{35} \mathrm{ONSi}$ $\left[\mathrm{M}+\mathrm{H}^{+}\right]$: 322.2566. Found: 322.2550.

( \pm )-1,2-anti-\{3-[(tert-Butyldimethylsilyl)oxy]pentan2-yl\}(decyl)amine (54): 71\% yield; yellowish oil; IR (film) $v_{\max } / \mathrm{cm}^{-1}: 2958,2928,2855,1463,1254,836 ;{ }^{1} \mathrm{H}$ NMR $\left(250 \mathrm{MHz}, \mathrm{CDCl}_{3}\right): \delta 3.50-3.61(\mathrm{~m}, 1 \mathrm{H}), 2.54-2.71(\mathrm{~m}$, $2 \mathrm{H}), 2.38-2.51(\mathrm{~m}, 1 \mathrm{H}), 1.38-1.60(\mathrm{~m}, 6 \mathrm{H}), 1.26(\mathrm{br}, 16 \mathrm{H})$, 0.96 (d, 3H, J 6.5 Hz), 0.81-0.91 (m, 12H), 0.05 (s, 6H); ${ }^{13} \mathrm{C} \mathrm{NMR}\left(62.5 \mathrm{MHz}, \mathrm{CDCl}_{3}\right): \delta 76.6,56.9,47.8,31.9,30.4$, 
29.6, 29.5, 29.3, 27.5, 25.8, 25.2, 22.6, 18.1, 15.1, 14.1, 10.5, -4.4, -4.42; HRMS (ESI TOF) Calcd. for $\mathrm{C}_{21} \mathrm{H}_{47} \mathrm{NOSi}$ $\left[\mathrm{M}+\mathrm{H}^{+}\right]$: 358.3505 . Found: 358.3428 .

Synthesis of ( \pm$)-1-[($ tert-Butyldimethylsilyl)oxy]1-(3-chlorophenyl)propan-2-ol (60)

To a solution of acyloin $34(170 \mathrm{mg}, 0.57 \mathrm{mmol})$ in anhydrous methanol $(10 \mathrm{~mL})$, under argon atmosphere and at $0{ }^{\circ} \mathrm{C}$ was added $\mathrm{NaBH}_{4}(108.3 \mathrm{mg}, 2.85 \mathrm{mmol})$. The resulting mixture was stirred for $10 \mathrm{~min}$, after that $1 \mathrm{~mL}$ of water was added and the solvent was removed under reduced pressure. The residue was diluted with ethyl acetate $(15 \mathrm{~mL})$ and the organic phase was washed with brine $(5 \mathrm{~mL})$, dried over anhydrous $\mathrm{Na}_{2} \mathrm{SO}_{4}$ and the solvent was removed under vacuum to provide the alcohol $\mathbf{6 0}$, in almost quantitative yield. Thus, it was used to the next step without purification. Colorless oil; IR (film) $v_{\max } / \mathrm{cm}^{-1}: 3448$, 2949, 2933, 2851, 1462, 1254, 1070, 833, 775; ${ }^{1} \mathrm{H}$ NMR $\left(250 \mathrm{MHz}, \mathrm{CDCl}_{3}\right): \delta$ (mixture of diastereomers) 7.12-7.35 $(\mathrm{m}, 6 \mathrm{H}), 4.52\left(\mathrm{~d}, J 4.7 \mathrm{~Hz}, \mathrm{H}_{\mathrm{a}}{ }^{\prime}\right), 4.28\left(\mathrm{~d}, J 6.5 \mathrm{~Hz}, \mathrm{H}_{\mathrm{a}}\right), 3.80$ $\left(\mathrm{q}, J 4.7 \mathrm{~Hz}, \mathrm{H}_{\mathrm{b}}{ }^{\prime}\right), 3.70\left(\mathrm{q}, J 6.5 \mathrm{~Hz}, \mathrm{H}_{\mathrm{b}}\right), 2.36(\mathrm{~s}, 1 \mathrm{H}), 1.06$ $\left(\mathrm{d} 3 \mathrm{H},, J 6.5 \mathrm{~Hz}, \mathrm{C}_{3}{ }^{\prime}\right), 1.00,\left(\mathrm{~d}, 3 \mathrm{H}, J 6.5 \mathrm{~Hz}, \mathrm{C}_{3}\right), 0.89$ (s, 9H), 0.05 (s, 3H), -0.12 (s, 3H); ${ }^{13} \mathrm{C}$ NMR $(75.4 \mathrm{MHz}$, $\mathrm{CDCl}_{3}$ ): $\delta$ (diastereomeric mixture) 143.7, 143.3, 134.1, 133.9, 129.5, 129.3, 127.9, 127.7, 127.1, 125.2, 125.1, 80.0, 78.2, 72.4, 71.9, 25.8, 18.2, 18.1, 17.9, 17.6, -4.9; HRMS (ESI TOF) Calcd. for $\mathrm{C}_{15} \mathrm{H}_{25} \mathrm{ClO}_{2} \mathrm{Si}\left[\mathrm{M}+\mathrm{H}^{+}\right]$: 301.1391 . Found: 301.1577.

Synthesis of ( \pm )-1-(3-chlorophenyl)propane-1,2-diol (61)

To a solution of alcohol $( \pm)-60(100 \mathrm{mg}, 0.34 \mathrm{mmol})$ in $\operatorname{THF}(6 \mathrm{~mL})$ at $0^{\circ} \mathrm{C}$ was added a solution of TBAF $(0.41 \mathrm{~mL}$, $1 \mathrm{~mol} \mathrm{~L}^{-1}$ in toluene). The mixture was stirred for $5 \mathrm{~min}$ and was warmed to room temperature. After $2 \mathrm{~h}$, a mixture of water $(5 \mathrm{~mL})$ and ethyl acetate $(15 \mathrm{~mL})$ was added to the reaction medium. The organic phase was separated, washed with brine $(5 \mathrm{~mL})$, dried over anhydrous $\mathrm{Na}_{2} \mathrm{SO}_{4}$ and the solvent was removed under vacuum. The residue was filtered through silica gel (solvent: ethyl acetate) to provide the diol ( \pm )-61, in $97 \%$ yield. Colorless oil; IR (film) $v_{\max } / \mathrm{cm}^{-1}$ : 3395, 2978, 2876, 1569, 1426, 1082, 1037, 780, 694; ${ }^{1} \mathrm{H}$ NMR $\left(250 \mathrm{MHz}, \mathrm{CDCl}_{3}\right): \delta$ (diastereomeric mixture) 7.16-7.39 (m, 6H), $4.67\left(\mathrm{~d}, J 4.0 \mathrm{~Hz}, \mathrm{H}_{\mathrm{a}}{ }^{\prime}\right), 4.33(\mathrm{~d}$, $\left.J 7.1 \mathrm{~Hz}, \mathrm{H}_{\mathrm{a}}\right), 3.92-4.07\left(\mathrm{~m}, \mathrm{H}_{\mathrm{b}}{ }^{\prime}\right), 3.81\left(\mathrm{q}, J 7.1 \mathrm{~Hz}, \mathrm{H}_{\mathrm{b}}\right), 2.62$ (s, $3 \mathrm{H}, \mathrm{O} \underline{\mathrm{H}}), 1.00-1.13\left(\mathrm{~m}, 5 \mathrm{H}, \mathrm{C}_{3}\right) ;{ }^{13} \mathrm{C} \mathrm{NMR}(62.5 \mathrm{MHz}$, $\mathrm{CDCl}_{3}$ ): $\delta$ (diastereomeric mixture) 143.1, 142.4, 134.4, 129.7, 129.5, 128.2, 127.8, 127.0, 126.7, 125.1, 124.8, 78.6, 77.2, 71.9, 71.0, 18.7, 16.9; HRMS (ESI TOF) Calcd. for $\mathrm{C}_{9} \mathrm{H}_{11} \mathrm{O}_{2} \mathrm{Cl}\left[\mathrm{M}+\mathrm{Na}^{+}\right]$: 209.6252. Found: 209.6223.
Synthesis of ( \pm )-1-(3-chlorophenyl)-2-hydroxypropan1-one (59)

To a solution of diol $( \pm)-61(70 \mathrm{mg}, 0.38 \mathrm{mmol})$ in DMSO (1 mL) was slowly added 45\% IBX $(236.4 \mathrm{mg}$, $0.38 \mathrm{mmol})$ at room temperature. Caution: addition time $15 \mathrm{~h}$, faster addition will give a diketone product. The mixture was stirred for $30 \mathrm{~h}$. After that, the reaction medium was diluted with water $(5 \mathrm{~mL})$ and ethyl acetate $(15 \mathrm{~mL})$. The organic phase was separated, washed with brine $(5 \mathrm{~mL})$, dried over anhydrous $\mathrm{Na}_{2} \mathrm{SO}_{4}$ and the solvent was removed under vacuum. The residue was purified by silica gel column chromatography (solvent: hexanes:ethyl acetate, up to 60:40) to provide the acyloin $( \pm)-59$, in $85 \%$ yield. Yellowish oil; IR (film) $v_{\max } / \mathrm{cm}^{-1}: 3436,2982,2921$, 2843, 1687, 1569, 1262, 1123, 1070, 1033, 739; ${ }^{1} \mathrm{H}$ NMR $\left(250 \mathrm{MHz}, \mathrm{CDCl}_{3}\right): \delta 7.91(\mathrm{~s}, 1 \mathrm{H}), 7.77-7.82(\mathrm{~m}, 1 \mathrm{H})$, 7.57-7.62 (m, 1H), $7.46(\mathrm{t}, 1 \mathrm{H}, J 7.9 \mathrm{~Hz}), 5.12(\mathrm{q}, 1 \mathrm{H}$, $J 7.0 \mathrm{~Hz}, \mathrm{C} \underline{\mathrm{H}}), 1.45\left(\mathrm{~d}, 3 \mathrm{H}, J 7.0 \mathrm{~Hz}, \mathrm{C}_{3}\right) ;{ }^{13} \mathrm{C} \mathrm{NMR}$ (75.4 MHz, $\mathrm{CDCl}_{3}$ ): $\delta$ 201.3, 135.3, 134.9, 133.9, 130.2, 128.7, 126.6, 77.2, 69.5, 22.1; HRMS (ESI TOF) Calcd. for $\mathrm{C}_{9} \mathrm{H}_{9} \mathrm{O}_{2} \mathrm{Cl}\left[\mathrm{M}+\mathrm{Na}^{+}\right]$: 207.6093. Found: 207.6075.

Synthesis of ( \pm )-2-(tert-butylamino)-1-(3-chlorophenyl) propan-1-one (bupropion) (1)

To a solution of acyloin $( \pm)-\mathbf{5 9}(30 \mathrm{mg}, 0.16 \mathrm{mmol})$ in $3 \mathrm{~mL}$ of anhydrous $\mathrm{CH}_{2} \mathrm{Cl}_{2}$, at $-78{ }^{\circ} \mathrm{C}$, was added triflic anhydride $(50 \mathrm{mg}, 0.177 \mathrm{mmol}, 29.81 \mu \mathrm{L}$ ) and 2,6-lutidine (26 mg, $0.24 \mathrm{mmol}, 28.26 \mu \mathrm{L}$ ). The resulting mixture was cooled to $-40{ }^{\circ} \mathrm{C}$ and stirred for $30 \mathrm{~min}$. After that, the organic phase was washed with brine $(5 \mathrm{~mL})$, dried over anhydrous $\mathrm{Na}_{2} \mathrm{SO}_{4}$ and the solvent was removed under vacuum. The residue was immediately dissolved in dry $\mathrm{CH}_{2} \mathrm{Cl}_{2}$ and the solution was cooled to $-40{ }^{\circ} \mathrm{C}$. Then tert-butylamine ( $29.6 \mathrm{mg}, 0.41 \mathrm{mmol}, 42.5 \mu \mathrm{L}$ ) was added. The mixture was stirred for $2 \mathrm{~h}$ at $-40^{\circ} \mathrm{C}$, warmed to $0{ }^{\circ} \mathrm{C}$ and kept at this final temperature for $12 \mathrm{~h}$. After, the reaction medium was diluted with dichloromethane $(10 \mathrm{~mL})$. The organic phase was separated, washed with sodium bicarbonate $(5 \mathrm{~mL})$, water $(5 \mathrm{~mL})$, brine $\left(5 \mathrm{~mL}\right.$ ), dried over anhydrous $\mathrm{Na}_{2} \mathrm{SO}_{4}$ and the solvent was removed under reduced pressure. The residue was purified through silica gel (solvent: hexanes:ethyl acetate, up to $60: 40$ ) to provide ( \pm )-1 as a white solid, in $75 \%$ yield; $\mathrm{mp} 233-234{ }^{\circ} \mathrm{C}$ (as hydrochloride; ${ }^{41}$ 233-234 $\left.{ }^{\circ} \mathrm{C}\right) ;{ }^{1} \mathrm{H}$ NMR $\left(250 \mathrm{MHz}, \mathrm{CDCl}_{3}\right): \delta$ 7.83-7.90 (m, 1H), 8.03-8.11 (m, 1H), 7.97-7.99 (m, 1H), 6.11

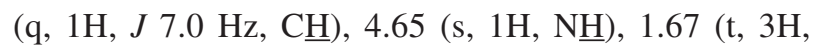
$\left.J 7.0 \mathrm{~Hz}, \mathrm{C}_{3}\right), 1.44(\mathrm{~s}, 9 \mathrm{H}) ;{ }^{13} \mathrm{C} \mathrm{NMR}\left(62.5 \mathrm{MHz}, \mathrm{CDCl}_{3}\right)$ : $\delta$ 195.7, 136.0, 135.2, 133.5, 130.1, 128.6, 126.5, 71.9, 
58.1, 30.3, 17.0; HRMS (ESI TOF) Calcd. for $\mathrm{C}_{13} \mathrm{H}_{18} \mathrm{OCIN}$ $\left[\mathrm{M}+\mathrm{Na}^{+}\right]:$262.7309. Found: 262.7299 .

Synthesis of ( \pm )-1,2-anti-benzyl(\{3-[(tertbutyldimethylsilyl)oxy]octadecan-2-yl\})amine (62)

To a stirred mixture of acyloin $( \pm)-\mathbf{4 2}(0.2 \mathrm{~g}, 0.502 \mathrm{mmol})$ and molecular sieves ( $4 \AA$ ) in anhydrous dichloromethane ( $3 \mathrm{~mL}$ ), under an argon atmosphere, was added distilled benzylamine $(0.268 \mathrm{~g}, 0.27 \mathrm{~mL}, 2.51 \mathrm{mmol})$. The resulting mixture was kept at $40{ }^{\circ} \mathrm{C}$ for $7 \mathrm{~h}$. Then, the reaction was cooled to $0{ }^{\circ} \mathrm{C}$ and $\mathrm{NaBH}_{3} \mathrm{CN}(0.038 \mathrm{~g}, 0.6 \mathrm{mmol})$ was added in three small portions at $40 \mathrm{~min}$. intervals. The reaction was warmed to room temperature and stirred for $12 \mathrm{~h}$. The solvent was removed under reduced pressure and the residue was dissolved with ethyl acetate. The organic phase was filtered, washed with distilled water $(3 \times 10 \mathrm{~mL})$, brine $(15 \mathrm{~mL})$, dried over anhydrous sodium sulfate and evaporated. The crude residue was purified by silica gel column chromatography (ethyl acetate:hexane, 25:75) to provide anti aminoalcohol $\mathbf{6 2}(0.19 \mathrm{~g})$, as a viscous colorless oil, in $70 \%$ yield; IR (film) $\mathrm{v}_{\max } / \mathrm{cm}^{-1}: 2925$, 2854, 1463, 1254, 1030; ${ }^{1} \mathrm{H}$ NMR (250 MHz, $\mathrm{CDCl}_{3}$ ): d 7.22-7.32 (m, 5H), $3.89(\mathrm{~d}, 1 \mathrm{H}, J 13.2 \mathrm{~Hz}), 3.67(\mathrm{~d}, 1 \mathrm{H}$, $J 13.2 \mathrm{~Hz}), 3.54(\mathrm{q}, 1 \mathrm{H}, J 4.6 \mathrm{~Hz}), 2.69$ (q, $1 \mathrm{H}, J 6.0 \mathrm{~Hz})$, $1.32-1.48(\mathrm{~m}, 2 \mathrm{H}), 1.17-1.35(\mathrm{~m}, 26 \mathrm{H}), 1.02(\mathrm{~d}, 3 \mathrm{H}$, $J 6.5 \mathrm{~Hz}), 0.82-0.92(\mathrm{~m}, 12 \mathrm{H}), 0.04(\mathrm{~s}, 3 \mathrm{H}),-0.02(\mathrm{~s}, 3 \mathrm{H})$; ${ }^{13} \mathrm{C}$ NMR $\left(62.5 \mathrm{MHz}, \mathrm{CDCl}_{3}\right): \delta 140.6,128.3,128.14$, $128.1,126.8,75.4,56.3,51.5,32.1,31.9,29.8,29.7$, 29.65, 29.6, 29.4, 26.1, 25.9, 22.7, 18.1, 15.2, 14.1, -4.3, -4.4; HRMS (ESI TOF) Calcd. for $\mathrm{C}_{31} \mathrm{H}_{59} \mathrm{NOSi}[\mathrm{M}+\mathrm{H}]^{+}$: 490.4439. Found: 490.4434.

Synthesis of ( \pm )-1,2-anti-[(2-aminooctadecan-3-yl)oxy] (tert-butyl)dimethylsilane (63)

To a stirred solution of aminoalcohol ( \pm )-62 (0.025 g, $0.05 \mathrm{mmol})$ in a mixture of dichloromethane $(1.5 \mathrm{~mL})$ and acetic acid $(1.0 \mathrm{~mL})$ was added $10 \% \mathrm{Pd} / \mathrm{C}(0.006 \mathrm{~g})$. The resulting suspension was purged twice with hydrogen gas and kept under a hydrogen atmosphere. Then, the reaction was warmed at $50{ }^{\circ} \mathrm{C}$ and stirred for $20 \mathrm{~h}$ under hydrogen atmosphere. After that time, the reaction mixture was diluted with dichloromethane $(10 \mathrm{~mL})$ and the organic phase was washed with a saturated solution of $\mathrm{NaHCO}_{3}$ $(8 \mathrm{~mL})$, dried over anhydrous sodium sulfate and evaporated. The crude residue was filtered over a plug of silica gel (dichloromethane:methanol, 97:3, as eluent) to provide silylated aminoalcohol $( \pm)-63(0.018 \mathrm{~g})$, as a viscous colorless oil, in $90 \%$ yield; IR (film) $v_{\max } / \mathrm{cm}^{-1}: 3409,2926$, 2855, 1682, 1465, 1255, 1084; ${ }^{1} \mathrm{H}$ NMR (250 MHz, $\mathrm{CDCl}_{3}$ ): $\delta 3.45-3.60(\mathrm{~m}, 1 \mathrm{H}), 2.85-2.99(\mathrm{~m}, 1 \mathrm{H}), 1.10-1.45(\mathrm{~m}, 28 \mathrm{H})$, 1.01 (d, 3H, J6.6 Hz), 0.80-0.91 (m, 12H), 0.0-0.10 (m, 6H); ${ }^{13} \mathrm{C} \mathrm{NMR}\left(62.5 \mathrm{MHz}, \mathrm{CDCl}_{3}\right): \delta 77.2,56.3,50.8,31.9,31.6$, 29.8, 29.7, 29.6, 29.3, 25.9, 25.7, 22.7, 18.1, 17.9, 14.1, -4.3, -4.4; HRMS (ESI TOF) Calcd. for $\mathrm{C}_{24} \mathrm{H}_{53} \mathrm{NOSi}[\mathrm{M}+\mathrm{H}]^{+}$: 400.3964. Found: 400.3960.

Synthesis of (土)-1,2-anti-2-aminooctadecan-3-ol (spisulosine) (2)

To a stirred solution of aminoalcohol $( \pm)-63(0.01 \mathrm{~g}$, $0.025 \mathrm{mmol})$ in a mixture of dichloromethane $(0.5 \mathrm{~mL})$ and methanol $(0.5 \mathrm{~mL})$ was added a solution of concentrated hydrochloric acid $(0.1 \mathrm{~mL})$. The resulting mixture was warmed to $50{ }^{\circ} \mathrm{C}$ and stirred for $20 \mathrm{~h}$. Then, the solvents were removed under reduced pressure and the residue was dissolved in dichloromethane $(5 \mathrm{~mL})$. To the stirred organic phase was added a saturated sodium bicarbonate solution $(5 \mathrm{~mL})$ and the resulting mixture was vigorously stirred for $30 \mathrm{~min}$. The organic phase was separated, dried over anhydrous sodium sulfate and removed under reduced pressure. The crude residue was filtered over a plug of Florisil ${ }^{\circledR}$ to provide $( \pm)$-spisulosine $(\mathbf{1}, 7 \mathrm{mg})$, as a white solid, in 98\% yield; mp 58-59 ${ }^{\circ} \mathrm{C}$; IR (film) $\mathrm{v}_{\max } / \mathrm{cm}^{-1}$ : $3348,3297,2955,2919,2851,1607,1471 ;{ }^{1} \mathrm{HNMR}(250 \mathrm{MHz}$, $\left.\mathrm{CDCl}_{3}\right): \delta 3.39-3.47(\mathrm{~m}, 1 \mathrm{H}), 2.88-3.06(\mathrm{~m}, 1 \mathrm{H}), 1.64-1.88(\mathrm{bs}$, $3 \mathrm{H}), 1.20-1.40(\mathrm{~m}, 28 \mathrm{H}), 1.01(\mathrm{~d}, 3 \mathrm{H}, J 6.5 \mathrm{~Hz}), 0.88(\mathrm{t}, 3 \mathrm{H}$, $J 7.0 \mathrm{~Hz}) ;{ }^{13} \mathrm{C} \mathrm{NMR}\left(62.5 \mathrm{MHz}, \mathrm{CDCl}_{3}\right): \delta 74.7,50.3,32.4$, 31.9, 29.8, 29.7, 29.6, 29.3, 26.2, 22.7, 16.8, 14.1; HRMS (ESI TOF) Calcd. for $\mathrm{C}_{18} \mathrm{H}_{40} \mathrm{NO}[\mathrm{M}+\mathrm{H}]^{+}:$286.3110; Found 286.3088.

\section{Supplementary Information}

All the spectra of the synthesized compounds in this paper are available as supplementary file and are free of charge at http/jbcs.sbq.org.br as pdf file.

\section{Acknowledgments}

Authors thank Conselho Nacional de Desenvolvimento Científico e Tecnológico (CNPq) and Fundação de Amparo à Pesquisa do Estado de São Paulo (FAPESP) for financial support. G. W. A. and M. C. thank FAPESP for fellowships. The authors are grateful to Prof. C. H. Collins for English revision of this text.

\section{References}

1. Ikariya, T.; Gridnev, I. D.; Top. Catal. 2010, 53, 894; Krüger, K.; Tillack, A.; Beller, M.; ChemSusChem 2009, 2, 715; Narasaka, 
K.; Kitamura, M.; Eur. J. Org. Chem. 2005, 4505; Dembech, P.; Seconi, G.; Ricci, A.; Chem. Eur. J. 2000, 6, 1281. For some examples of formation of $\mathrm{C}-\mathrm{N}$ bond catalyzed by transition metals, see: Surry, D. S.; Buchwald, S. L.; Angew. Chem. Int. Ed. 2008, 47, 6338; Buchwald, S. L.; Jiang, L. In Metal-Catalyzed Cross-Coupling Reactions; deMeijere, A.; Diederich, F., eds.; Wiley-VCH: Weinheim, 2004, p. 699; Hartwig, J. F.; Acc. Chem. Res. 2008, 41, 1534; Marion, N.; Nolan, S. P.; Acc. Chem. Res. 2008, 41, 1440; Beccalli, E. M.; Broggini, G.; Martinelli, M.; Sottocornola, S.; Chem. Rev. 2007, 107, 5318; Ackerman, L.; Born, R.; Spatz, J. H.; Althammer, A.; Gschrei, C. J.; Pure Appl. Chem. 2006, 78, 209; Lakshman, M. K.; Curr. Org. Synth. 2005, $2,83$.

2. For a complete and recent review regarding electrophilic amination, see: Ciganek, E.; Org. React. 2008, 72, 1; AmadorBedolla, C.; Salomón-Ferrer, R.; Lester Jr., W. A.; VázquezMartínez, J. A.; Aspuru-Guzik, A.; J. Chem. Phys. 2007, 126, 204308.

3. For some reviews, see: Yale, H. L.; Chem. Rev. 1943, 33, 209; Bauer, L.; Exner, O.; Angew. Chem. Int. Ed. Eng. 1974, 13, 376. For recent references concerning the Lossen rearrangement, see: Dubé, P.; Fine Nathel, N. F.; Vetelino, M.; Couturier, M.; Aboussafy, C. L.; Pichette, S.; Jorgensen, M. L.; Hardink, M.; Org. Lett. 2009, 11, 5622 and references cited therein; Vasantha, B.; Hemantha, H. P.; Sureshbabu, V. V.; Synthesis 2010, 2990; Ramon, F.; Prié, G.; Lecornué, F.; Papot, S.; Tetrahedron Lett. 2009, 50, 6800; Marzoni, G.; Varney, M. D.; Org. Process Res. Dev. 1997, $1,81$.

4. Gawley, R. E.; Org. React. 1988, 35, 14; Hoelderich, W. F.; Catal. Today 2000, 62, 115. For some recent examples about Beckman rarrangement, see: Song; L.; Chen, X.; Zhang, S.; Zhang, H.; Li, P.; Luo, G.; Liu, W.; Duan, W.; Wang, W.; Org. Lett. 2008, 10, 5489; Hashimoto, M.; Obora, Y.; Sakaguchi, S.; Ishii, Y.; J. Org. Chem. 2008, 73, 2894; Ramalingan, C.; Park, Y.-T.; J. Org. Chem. 2007, 72, 4536; Yamabe, S.; Tsuchida, N.; Yamazaki, S.; J. Org. Chem. 2005, 70, 10638; Chandrasekar, S.; Gopalaiah, K.; Tetrahedron Lett. 2003, 44, 755; de Luca, L.; Giacomelli, G.; Porcheddu, A.; J. Org. Chem. 2002, 67, 6272; Sharghi, H.; Hosseini, M.; Synthesis 2002, 1057.

5. Li, J. J.; Named Reactions, $4^{\text {th }}$ ed., Springer-Verlag: Berlin, 2009, p. 490 (doi: 10.1007/978-3-642-01053-8_229); Grecian, S.; Aubé, J. In Organic Azides: Synthesis and Applications; Bräsé, S.; Banert, K., eds.; WILEY-VCH Verlag GmbH \& Co. KgaA: Weinheim, 2010, chapter 7 (doi: 10.1002/9780470682517.ch7). For some recent examples of this reaction, see: Gorin, D. J.; Davies, N. R.; Toste, F. D.; J. Am. Chem. Soc. 2005, 127, 11260; Yao, L.; Aubé, J.; J. Am. Chem. Soc. 2007, 129, 2766.

6. For a recent review, see: Caron, S.; Dugger, R. W.; Gut Ruggeri, S.; Ragan, J. A.; Brown Rippin, D. H.; Chem. Rev. 2006, 106, 2943.

7. Bräse, S.; Gil, C.; Knepper, K.; Zimmermann, V.; Angew. Chem. Int. Ed. 2005, 44, 5188.
8. Am Ende, D. J.; De Vries, K. M.; Clifford, P. J.; Brenek, S. J.; Org. Process Res. Dev. 1998, 2, 382.

9. Lebel, H.; Leogane, O.; Org. Lett. 2006, 8, 5717; Lebel, H.; Leogane, O.; Org. Lett. 2005, 7, 4107; Yao, R.-S.; Wu, S.-H.; Jiang, L.; Deng, S.-S.; Yu, S.-X.; Res. Chem. Intermed. 2010, 36,523 .

10. Mateus, C. R.; Coelho, F.; J. Braz. Chem. Soc. 2005, 16, 386. For reviews about Morita-Baylis-Hillman reaction, see: Basavaiah, D., Reddy, B. S.; Badsara, S. S.; Chem. Rev. 2010, 110, 5447; Carrasco-Sanchez, V.; Simirgiotis, M. J.; Santos, L. S.; Molecules 2009, 14, 3989; Basavaiah, D.; Rao, K. V.; Reddy, R. J.; Chem. Soc. Rev. 2007, 36, 1581; Almeida, W. P.; Coelho, F.; Quim. Nova 2000, 23, 98.

11. Schräpler, U.; Rühlmann, K.; Chem. Ber. 1964, 97, 1983; Mori, K.; Nakahara, T.; Nozaki, H.; Can. J. Chem. 1969, 47, 3266. For an example of a benzoin type reaction catalyzed by nucleophilic carbene for the preparation of cyclic acyloin, see: Enders, D.; Niemeier, O.; Synlett 2004, 2111; Heck, R.; Henderson, A. P.; Köhler, B.; Rétey, J.; Golding, B. T.; Eur. J. Org. Chem. 2001, 2623.

12. For a comprehensive review on $\alpha$-hydroxylations, see: Davis, F. A.; Chen, B.-C. In Houben-Weyl: Stereoselective Synthesis, vol. E 21; Helmchen, G.; Hoffmann, R. W.; Mulzer, J.; Schaumann, E., eds.; Thieme Stuttgart: New York, 1996, p. 4497; Zhou, P.; Chen, B. A.; Davies, F. A. In Asymmetric Oxidation Reactions; Katsuki, T., ed.; Oxford University Press: Oxford, 2001, p. 128; Plietker, B.; Tetrahedron: Asymmetry 2005, 16, 3453.

13. Khan, F. A.; Dash, J.; Sahu, N.; Gupta, S.; Org. Lett. 2002, 4, 1015 and references cited therein; Hayakawa, R.; Sahara, T.; Shimizu, M.; Tetrahedron Lett. 2000, 41, 7939; Bornemann, S.; Crout, D. H. G.; Dalton, H.; Kren, V.; Lobell, M.; Dean, G.; Thomson, N.; Turner, M. M.; J. Chem. Soc., Perkin Trans. 1 1996, 425; Kawai, Y.; Hida, K.; Tsujimoto, M.; Kondo, S.; Kitano, K.; Nakamura, K.; Ohno, A.; Bull. Chem. Soc. Jpn. 1999, 72, 99.

14. Plietker, B.; Org. Lett. 2004, 6, 289; Plietker, B.; J. Org. Chem. 2004, 69, 8287; Zhang, Y.; Shen, Z.; Tasng, J.; Zhang, Y.; King, L.; Zhang, Y.; Org. Biomol. Chem. 2004, 4, 1478.

15. Christoffers, J.; Werner, T.; Under, S.; Frey, W.; Eur. J. Org. Chem. 2003, 425.

16. Ooi, T.; Ohmatsu, K.; Maruoka, K.; J. Am. Chem. Soc. 2007, 129, 2410; Ooi, T.; Sasito, A.; Maruoka, K.; J. Am. Chem. Soc. 2003, 125, 3220.

17. Dir, A.; Kulkarni, S. K.; Eur. J. Pharmacol. 2007, 568, 177.

18. Mooney, M. E.; Sofuoglu, M.; Expert Rev. Neurother. 2006, 6, 965.

19. Lohray, B. B.; Thombare, P. S.; Bhushan, V.; PINSA-A 2002, 68, 391 (CA 139:84775).

20. Demko, Z. P.; Bartsch, M.; Sharpless, K. B.; Org. Lett., 2000, 2, 2221; O’Brien, P.; Angew. Chem. Int. Ed. 1999, 38, 326; Tao, B.; Schlingloff, G.; Sharpless, K. B.; Tetrahedron Lett., 1998, 39, 2507. 
21. For a review, see: Bergmeier, S. C.; Tetrahedron 2000, 56, 2561.

22. For some recent examples for the preparation of 1,2-aminoalcohols, see: Shivani, B. P.; Chakaborti, A. J.; J. Org. Chem. 2007, 72, 3713; Azizi, N.; Saidi, M. R.; Org. Lett. 2005, 7, 3649; Heydari, A.; Mehrdad, M.; Maleki, A.; Ahmadi N.; Synthesis 2004, 1557; Azoulay, S.; Manabe, K.; Kobayashi, S.; Org. Lett. 2005, 7, 4593; Cepanec, I.; Litvic, M.; Mikuldas, H.; Bartolincic, A.; Vinkovic, V.; Tetrahedron 2003, 59, 2435; Chakraborti, A. K.; Rudrawar, S.; Kondaskar, A.; Org. Biomol. Chem. 2004, 2, 1277; Kim, H. Y.; Talukdar, A.; Cushman, M.; Org. Lett. 2006, 8, 1085; Shivarkar, A. B.; Gupte, S. P.; Chaudhari, R. V.; Synlett 2006, 1374; Donohoe, T. J.; Johnson, P. D.; Pye, R. J.; Org. Biomol. Chem. 2003, 1, 2025.

23. Amarante, G. W.; Cavallaro, M.; Coelho, F.; Tetrahedron Lett. 2010, 51, 2597.

24. Rinehart, K. L.; Fregeau, N. L.; Warwick, R. A.; Gravalos, D. G.; Avila, J.; Faircloth, G. T.; WO Patent 9952521 A 1999 (CA 131:295576); Aceña, J. L.; Adrio, J.; Cuevas, C.; Gallego, P.; Manzanares, I.; Munt, S.; Rodriguez, I.; WO Patent 0194357 A1 2001 (CA 136:19976).

25. Sanchez, A. M.; Malagarie-Cazenave, S.; Olea, N.; Vara, D.; Cuevas, C.; Diaz-Laviada, I.; Eur. J. Pharmacol. 2008, 584, 237.

26. For some examples concerning the synthetic usage of MoritaBaylis-Hillman adducts, see: Resende, P.; Paioti, P. H. S.; Coelho, F.; Synth. Commun. 2011, 41, 227; Trazzi, G.; André, M. F.; Coelho, F.; J. Braz. Chem. Soc. 2010, 21, 2327; Amarante, G. W.; Coelho, F.; Tetrahedron, 2010, 66, 6749; Rodrigues Jr., M. T.; Gomes, J. C.; Smith, J.; Coelho, F.; Tetrahedron Lett. 2010, 51, 4988; Pirovani, R. V.; Ferreira, B. R. V.; Coelho, F.; Synlett 2009, 2333; Fournier, J. F.; Reddy, B. V. S.; Corey, E. J.; Org. Lett. 2005, 7, 2699.

27. Almeida, W. P.; Coelho, F.; Tetrahedron Lett. 1998, 39, 8609; Coelho, F.; Almeida, W. P.; Mateus C. R.; Veronese, D.; Lopes, E. C. S.; Silveira, G. P. C.; Rossi, R. C.; Pavam, C. H.; Tetrahedron 2002, 58, 7437.

28. Liang, H.; Synlett 2008, 2554; Shioiri, T.; Ninomiya, K.; Yamada, S.; J. Am. Chem. Soc. 1972, 94, 6203; Chan, B. K.; Ciufolini, M. A.; J. Org. Chem. 2007, 72, 8489.

29. Winkler, C. K.; Stueckler, C.; Mueller, N. J.; Pressnitz, D.; Faber, K.; Eur. J. Org. Chem. 2010, 6354; Patel, R. N.; Curr. Org. Chem. 2006, 10, 1289; Demir, A. S.; Ayhan, P.; Sopaci, S. B.; Clean 2007, 35, 406; Adam, W.; Lazarus, M.; SahaMöller, C. R.; Schreier, P.; Acc. Chem. Res. 1999, 32, 837.

30. For recent reviews, see: Gomez, S.; Peters, J. A.; Maschmeyer, T.; Adv. Synth. Catal. 2002, 344, 1037; Tararov, V. I.; Börner, A.; Synlett 2005, 203; Abdel-Magid, A. F.; Mehrman, S. J.; Org. Process Res. Dev. 2006, 10, 971; Nugent, T. C.; El-Shazly, M.; Adv. Synth. Catal. 2010, 352, 753; Tripathi, R. P.; Verma, S. S.; Pandey, J.; Tiwari, V. K.; Curr. Org. Chem. 2008, 12, 1093; Hayes, K. S.; Appl. Catal., A 2001, 221, 187; Zatsepin, T. S.;
Stetsenko, D. A.; Gait, M. J.; Oretskaya, T. S.; Bioconjugate Chem. 2005, 16, 471; Antos, J. M.; Francis, M. B.; Curr. Opin. Chem. Biol. 2006, 10, 253.

31. For reviews on enzymatic reductive amination, see: Wildeman, S. D.; Sonke, T.; Schoemaker, H. E.; May, O.; Acc. Chem. Res. 2007, 40, 1260; Höhne, M.; Bornscheuer, U. T.; ChemCatChem 2009, 1, 42.

32. For an outstanding example concerning a homogeneous reductive amination reaction, see: Wang, C.; Pettman, A.; Basca, J.; Xiao, J.; Angew. Chem. Int. Ed. 2010, 49, 7548 and references cited therein.

33. Cabral, S.; Hulin, B.; Kawal, M.; Tetrahedron Lett. 2007, 48, 7134.

34. Futagawa, S.; Inui, T.; Shiba, T.; Bull. Chem. Soc. Jpn 1973, 46, 3308; Dias, L. C.; Fattori, J.; Perez, C. C.; de Oliveira, V. M.; Aguilar, A. M.; Tetrahedron 2008, 64, 5891.

35. Dias, L. C.; Ferreira, M. A. B.; Tormena, C. F.; J. Phys. Chem. A 2008, 112, 232.

36. For some examples of total synthesis of bupropion, see: Kelley, J. L.; Musso, D. L.; Boswell, G. E.; Soroko, F. E.; Cooper, B. R.; J. Med. Chem. 1996, 39, 347; Musso, D. L.; Mehth, N. B.; Soroko, F. E.; Ferris, R. M.; Hollingsworth, E. B.; Kenney, B. T.; Chirality 1993, 5, 495; Musso, D. L.; Mehth, N. B.; Soroko, F. E.; Bioorg. Med. Chem. Lett. 1997, 7, 1; Fang, Q. K.; Han, Z.; Grover, P.; Kessler, D.; Senanayake, C. H.; Wald, S. A.; Tetrahedron: Asymmetry 2000, 11, 3659; Amarante, G. W.; Rezende, P.; Cavallaro, M.; Coelho, F.; Tetrahedron Lett. 2008, 49, 3744.

37. Bernardelli, P.; Moradei, O. M.; Friedrich, D.; Yang, J.; Gallou, F.; Dyck, B. P.; Doskotch, R. W.; Lange, T.; Paquette, L. O.; J. Am. Chem. Soc. 2001, 123, 9021.

38. Miljkovic, M. In Carbohydrates: Synthesis, Mechanisms and Stereoeletronic Effects; Springer Science: New York, 2010, p. 108.

39. Lawrence, S. A.; Amines: Synthesis, Properties and Applications; Cambrigde University Press: Cambridge, 2004.

40. Yun, J. M.; Sim, T. B.; Hahm, H. S.; Lee, W. K.; Ha, H.-J.; J. Org. Chem. 2003, 68, 7675; Seguin, C.; Ferreira, F.; Botuha, C.; Chemla, F.; Perez-Luna, A.; J. Org. Chem. 2009, 74, 6986; Allepuz, A. C.; Badorrey, R.; Diaz-de-Villegas, M. D.; Galvez, J. A.; Eur. J. Org. Chem. 2009, 35, 6172.

41. Perrine, D. M.; Ross, J. T.; Nervi, S. J.; Zimmerman, R. H.; J. Chem. Educ. 2000, 77, 1479; Reddy, Y. T.; Reddy, P. N.; Reddy, M. N.; Rajitha, B.; Crooks, P. A.; Synth. Commun. 2010, 40, 1566.

Submitted: February 8, 2011 Published online: May 17, 2011

FAPESP has sponsored the publication of this article. 


\title{
Supplementary Information
}

\section{Hyphenating the Curtius Rearrangement with Morita-Baylis-Hillman Adducts: Synthesis of Biologically Active Acyloins and Vicinal Aminoalcohols}

\author{
Giovanni W. Amarante, Mayra Cavallaro and Fernando Coelho*
}

Laboratório de Síntese de Produtos Naturais e Fármacos, Instituto de Química, Universidade de Campinas, 13083-970 Campinas-SP, Brazil

\section{General}

The ${ }^{1} \mathrm{H}$ and ${ }^{13} \mathrm{C}$ spectra were recorded on Bruker at $250 \mathrm{MHz}$ and $62.5 \mathrm{MHz}$ respectively. The ${ }^{1} \mathrm{H}$ and ${ }^{13} \mathrm{C}$ spectra were also recorded on Inova instrument at $500 \mathrm{MHz}$ and $125 \mathrm{MHz}$, respectively. The high resolution mass spectra were recorded using a Q-TOF Micromass equipment (Waters, UK).<smiles>COC(=O)C(=O)C(O)c1ccccc1[N+](=O)[O-]</smiles>
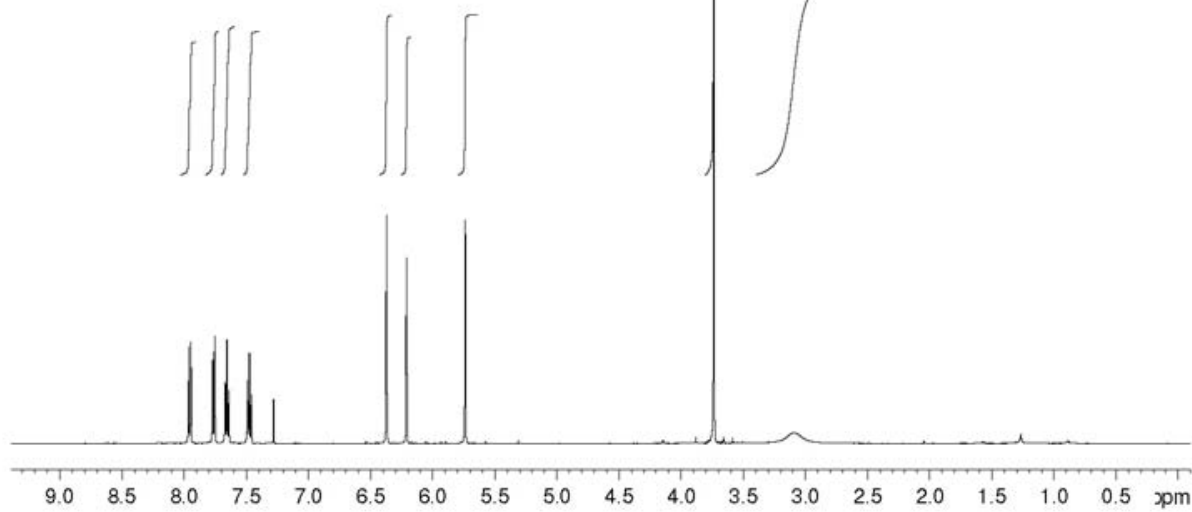

Figure S1. ${ }^{1} \mathrm{H}$ NMR $\left(\mathrm{CDCl}_{3}, 500 \mathrm{MHz}\right)$ of $\mathrm{MBH}$ adduct 3 . 


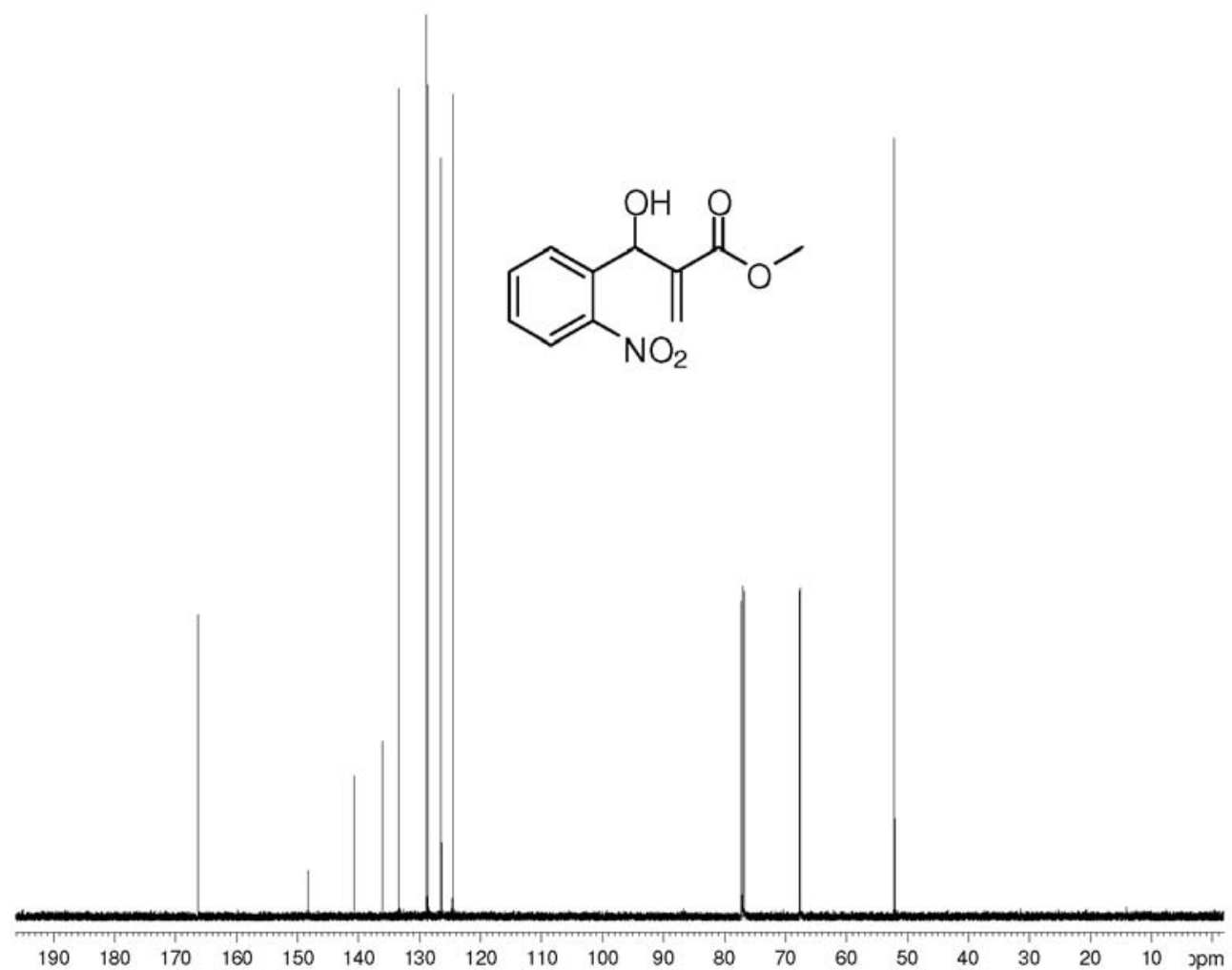

Figure S2. ${ }^{13} \mathrm{C}$ MMR $\left(\mathrm{CDCl}_{3}, 125 \mathrm{MHz}\right)$ of $\mathrm{MBH}$ adduct 3.

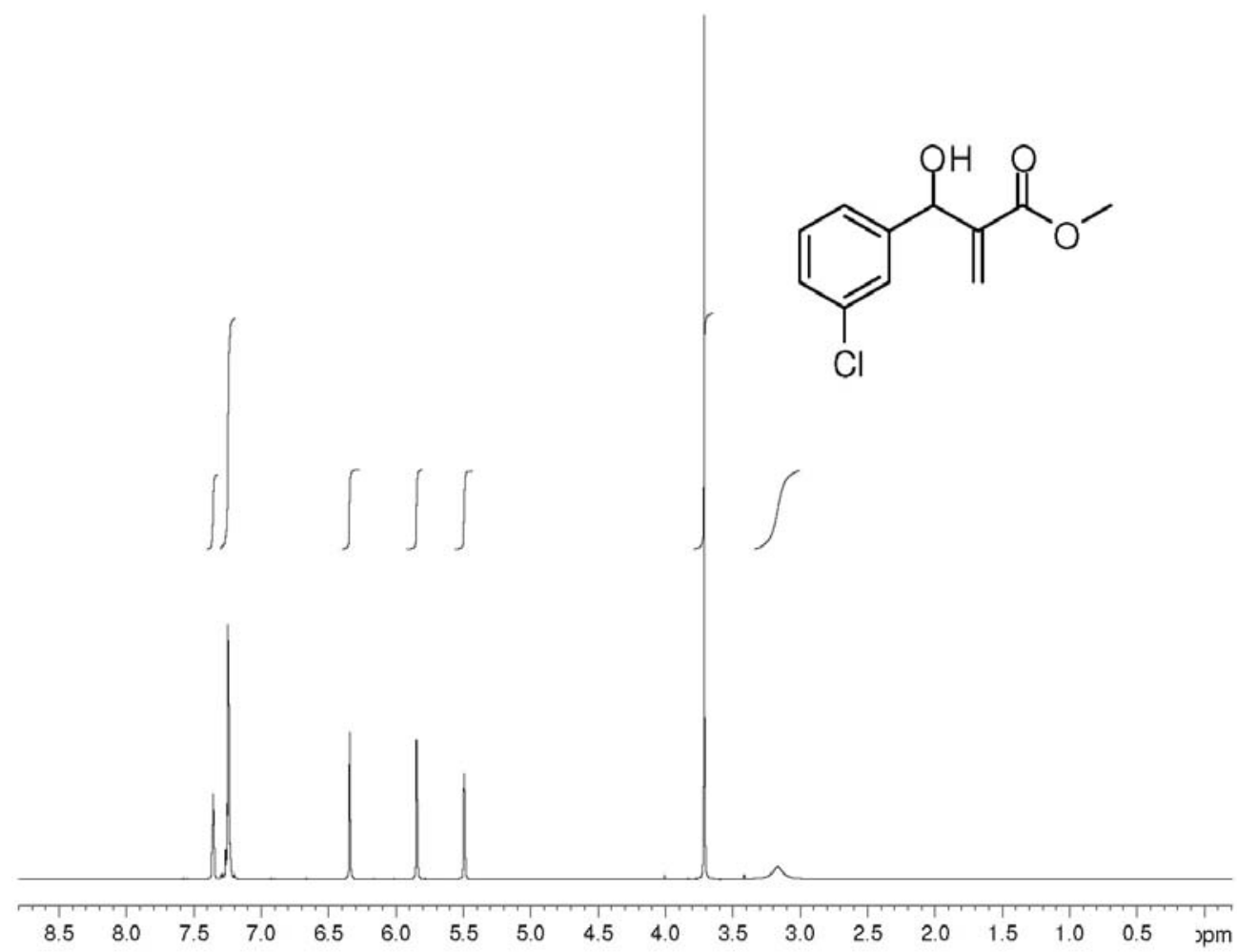

Figure S3. ${ }^{1} \mathrm{H} \mathrm{NMR}\left(\mathrm{CDCl}_{3}, 250 \mathrm{MHz}\right)$ of $\mathrm{MBH}$ adduct 4. 
<smiles>C=C(C(=O)OC)C(O)c1cccc(Cl)c1</smiles>

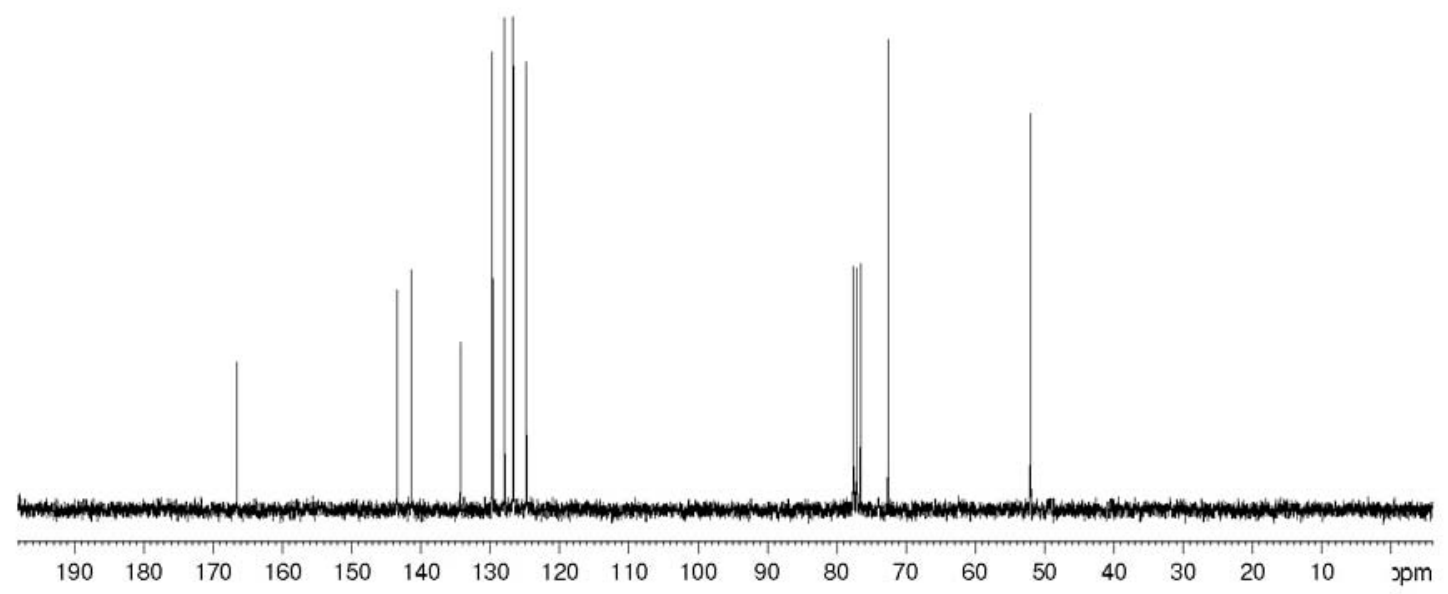

Figure S4. ${ }^{13} \mathrm{C} \mathrm{NMR}\left(\mathrm{CDCl}_{3}, 62.5 \mathrm{MHz}\right)$ of $\mathrm{MBH}$ adduct 4 .

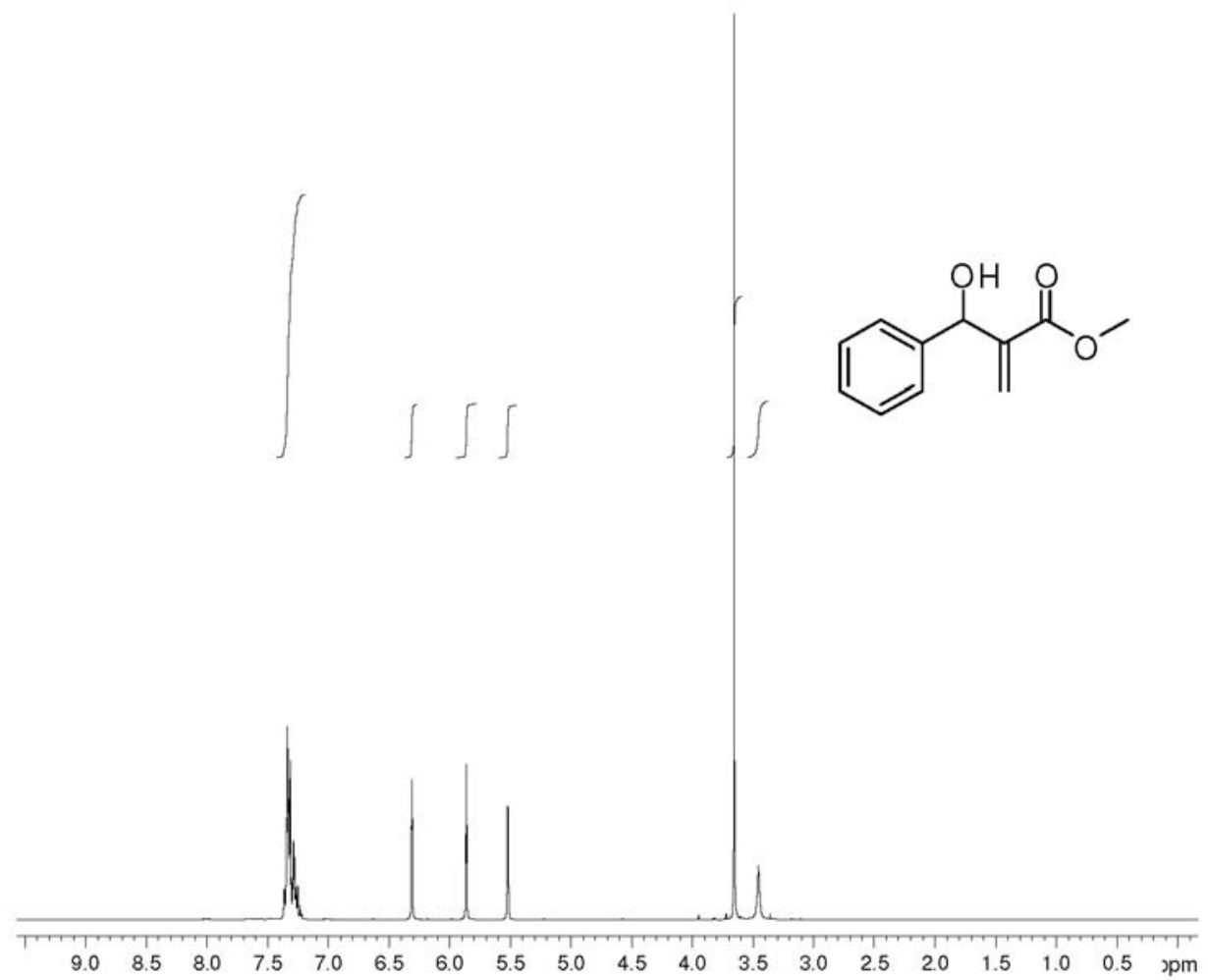

Figure S5. ${ }^{1} \mathrm{H} \mathrm{NMR}\left(\mathrm{CDCl}_{3}, 250 \mathrm{MHz}\right)$ of $\mathrm{MBH}$ adduct 5. 


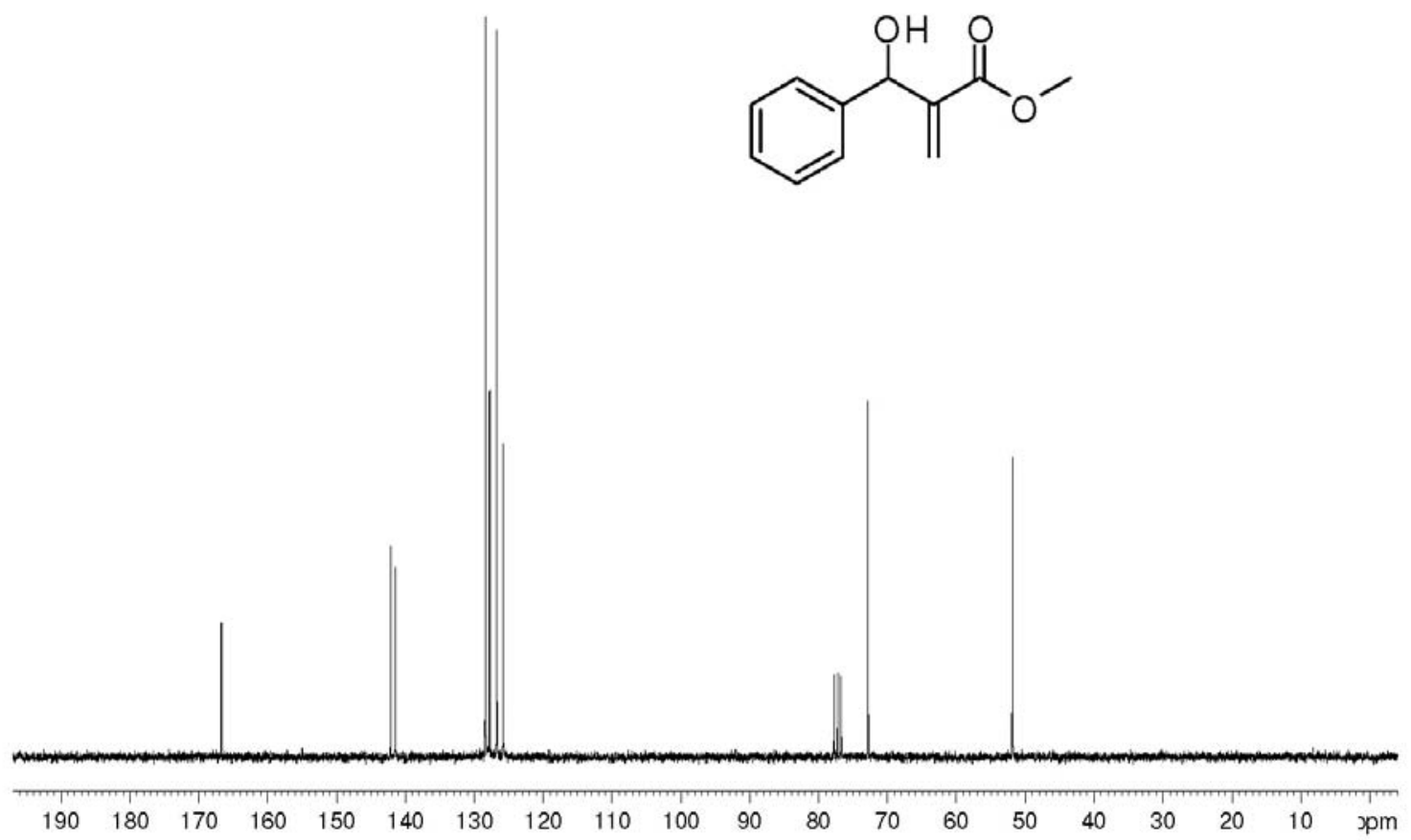

Figure S6. ${ }^{13} \mathrm{C} \mathrm{NMR}\left(\mathrm{CDCl}_{3}, 62.5 \mathrm{MHz}\right)$ of $\mathrm{MBH}$ adduct 5.

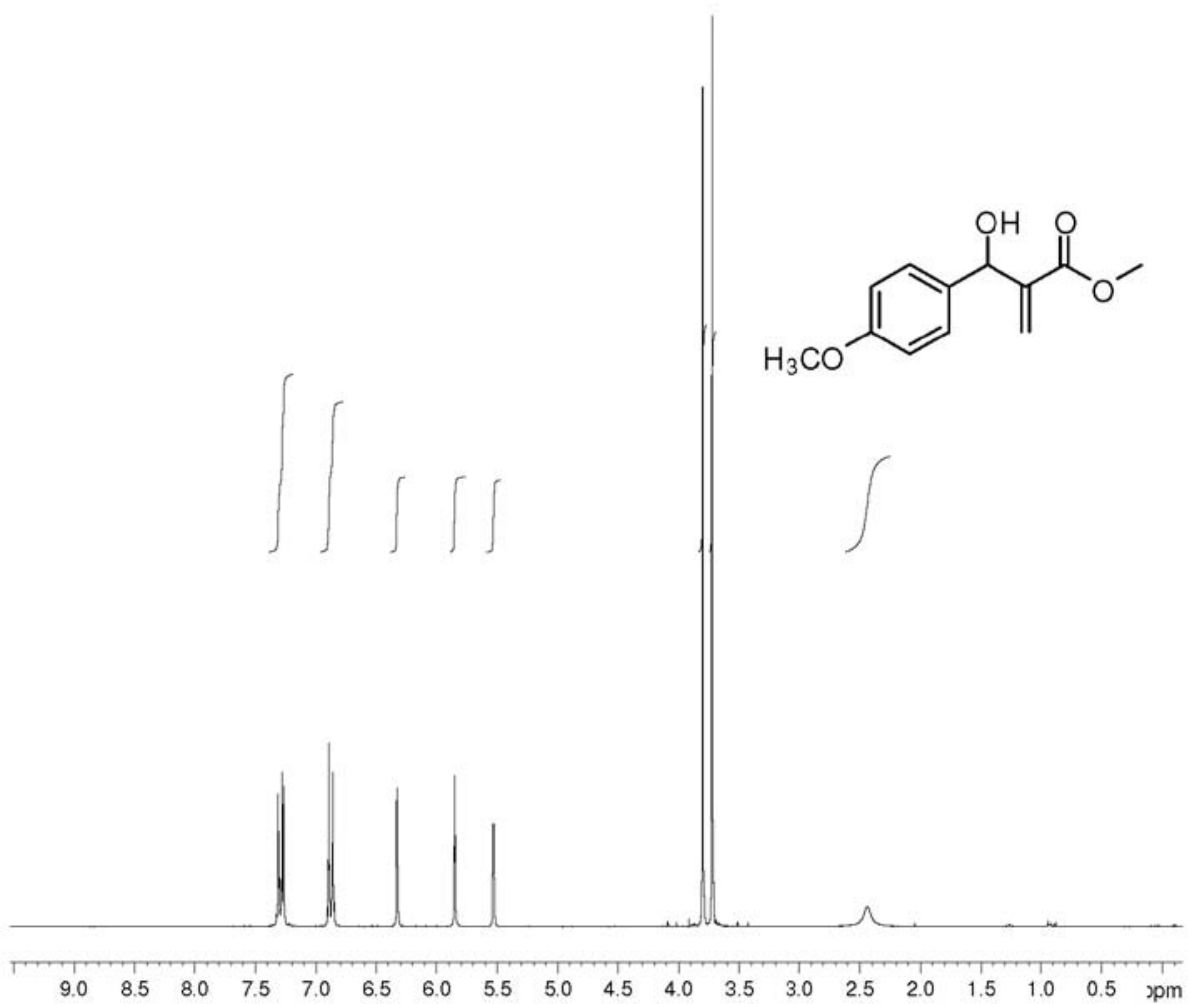

Figure S7. ${ }^{1} \mathrm{H} \mathrm{NMR}\left(\mathrm{CDCl}_{3}, 250 \mathrm{MHz}\right)$ of $\mathrm{MBH}$ adduct 6. 


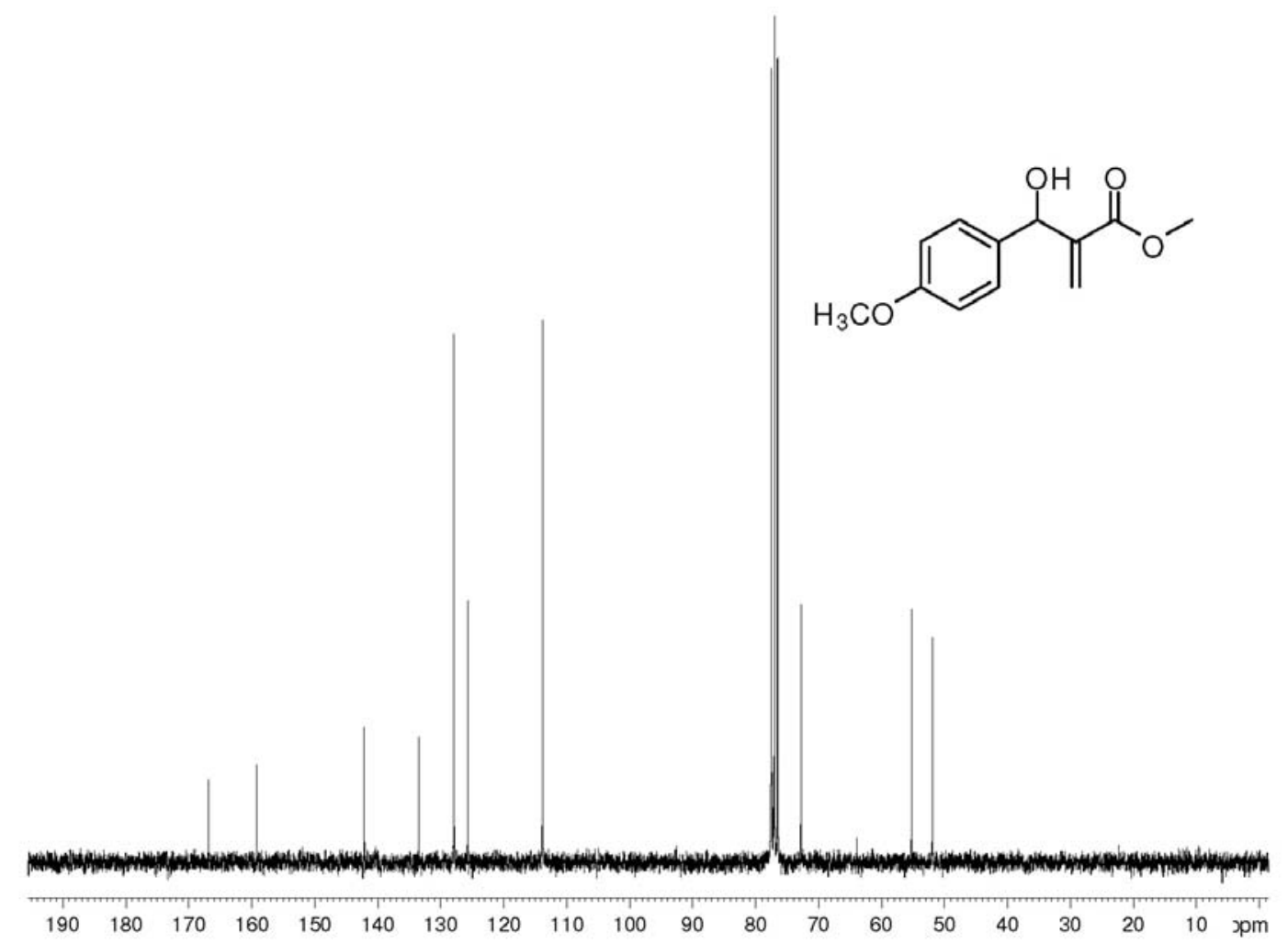

Figure S8. ${ }^{13} \mathrm{C} \mathrm{NMR}\left(\mathrm{CDCl}_{3}, 62.5 \mathrm{MHz}\right)$ of $\mathrm{MBH}$ adduct 6 .

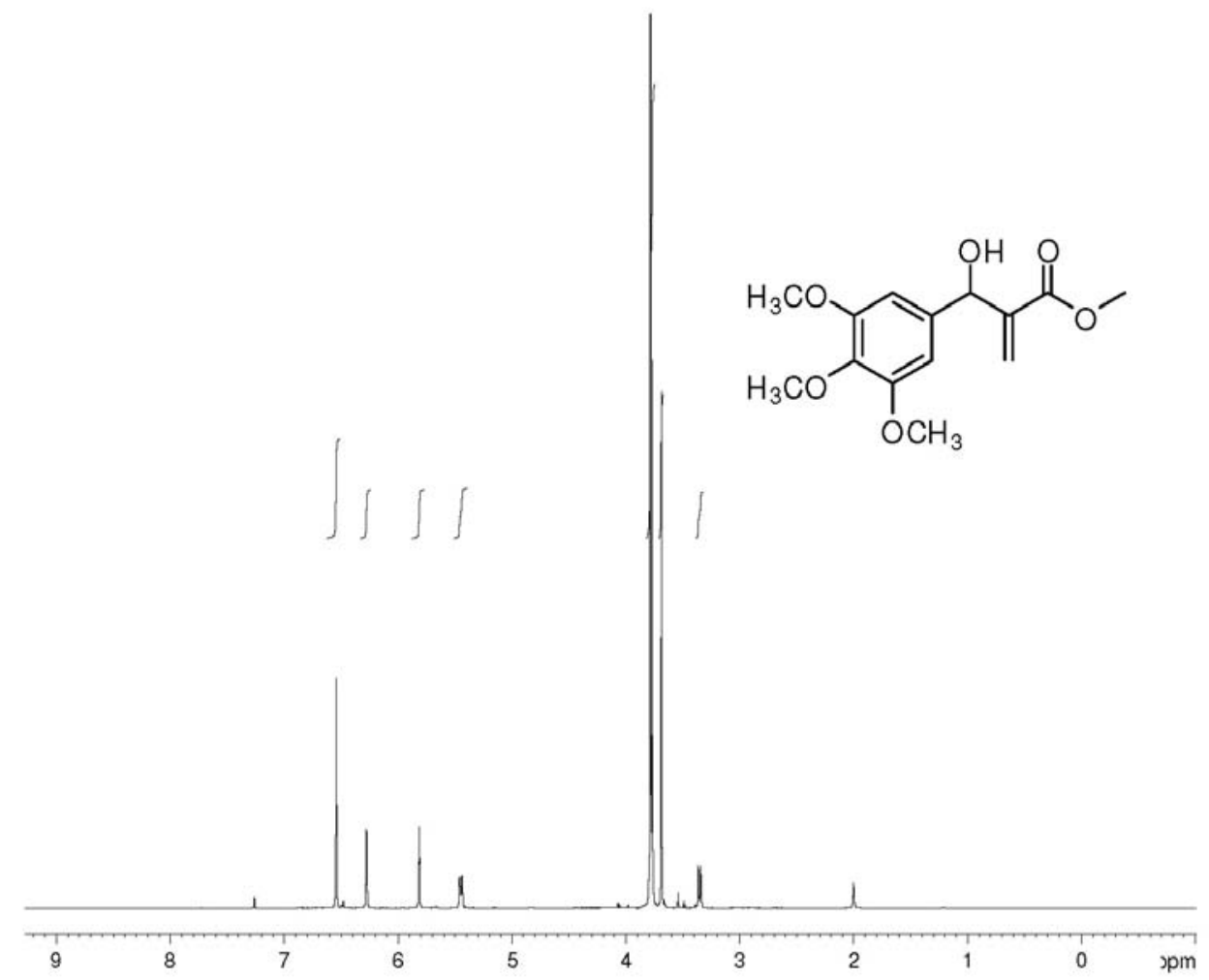

Figure S9. ${ }^{1} \mathrm{H} \mathrm{RMN}\left(\mathrm{CDCl}_{3}, 250 \mathrm{MHz}\right)$ of $\mathrm{MBH}$ adduct 7. 
<smiles>C=C(C(=O)OC)C(O)c1cc(OC)c(OC)c(OC)c1</smiles>

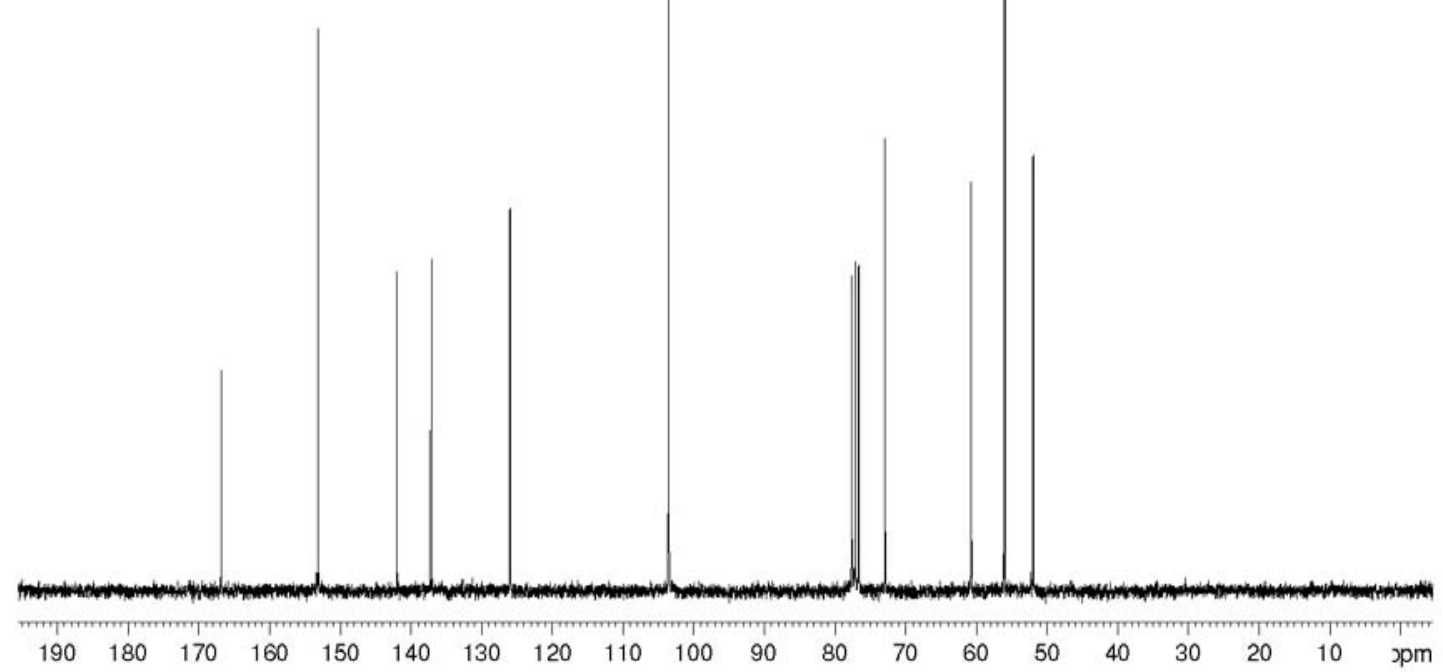

Figure S10. ${ }^{13} \mathrm{C}$ NMR $\left(\mathrm{CDCl}_{3}, 62.5 \mathrm{MHz}\right)$ of $\mathrm{MBH}$ adduct 7.

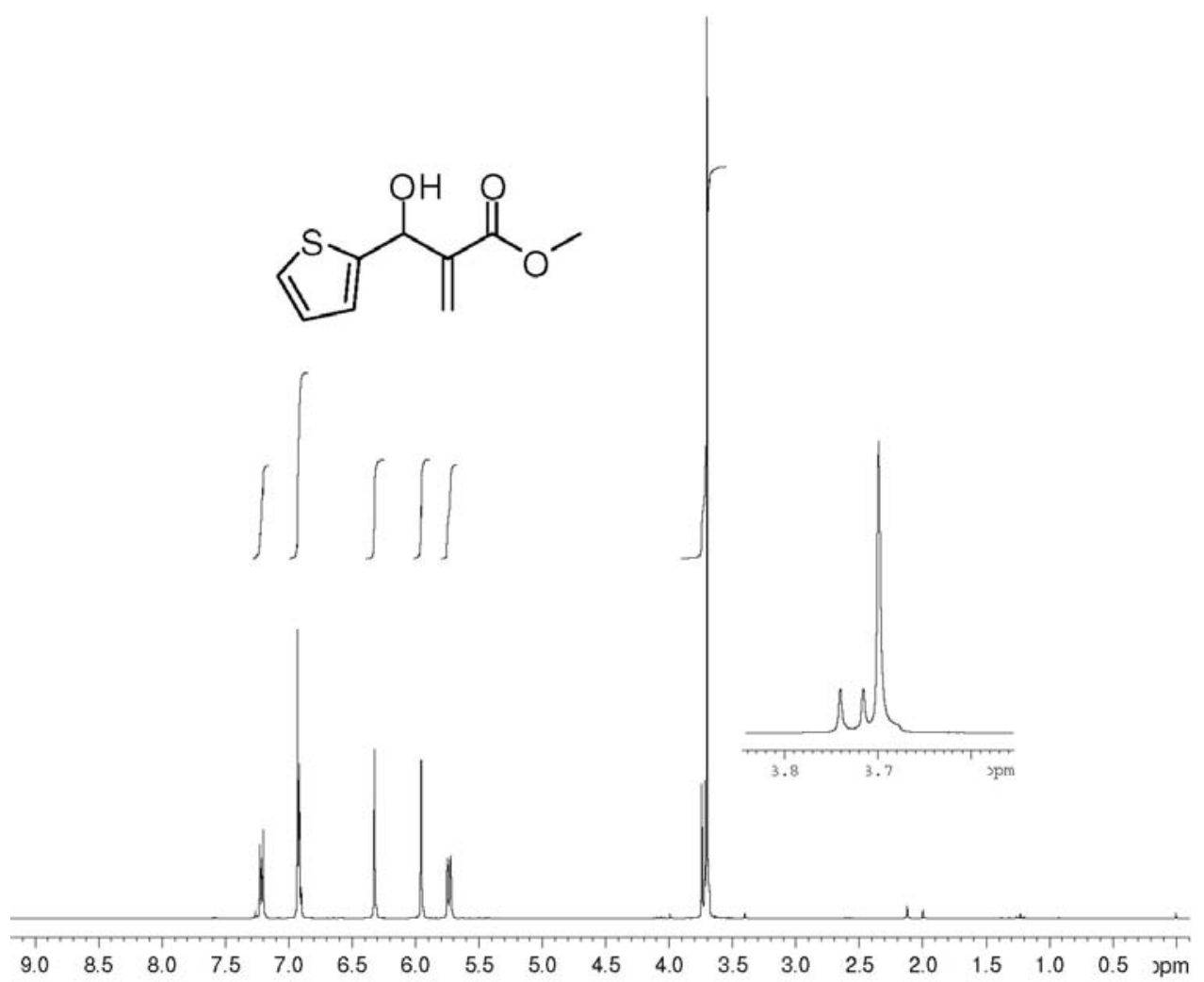

Figure S11. ${ }^{1} \mathrm{H}$ NMR $\left(\mathrm{CDCl}_{3}, 250 \mathrm{MHz}\right)$ of $\mathrm{MBH}$ adduct 8. 


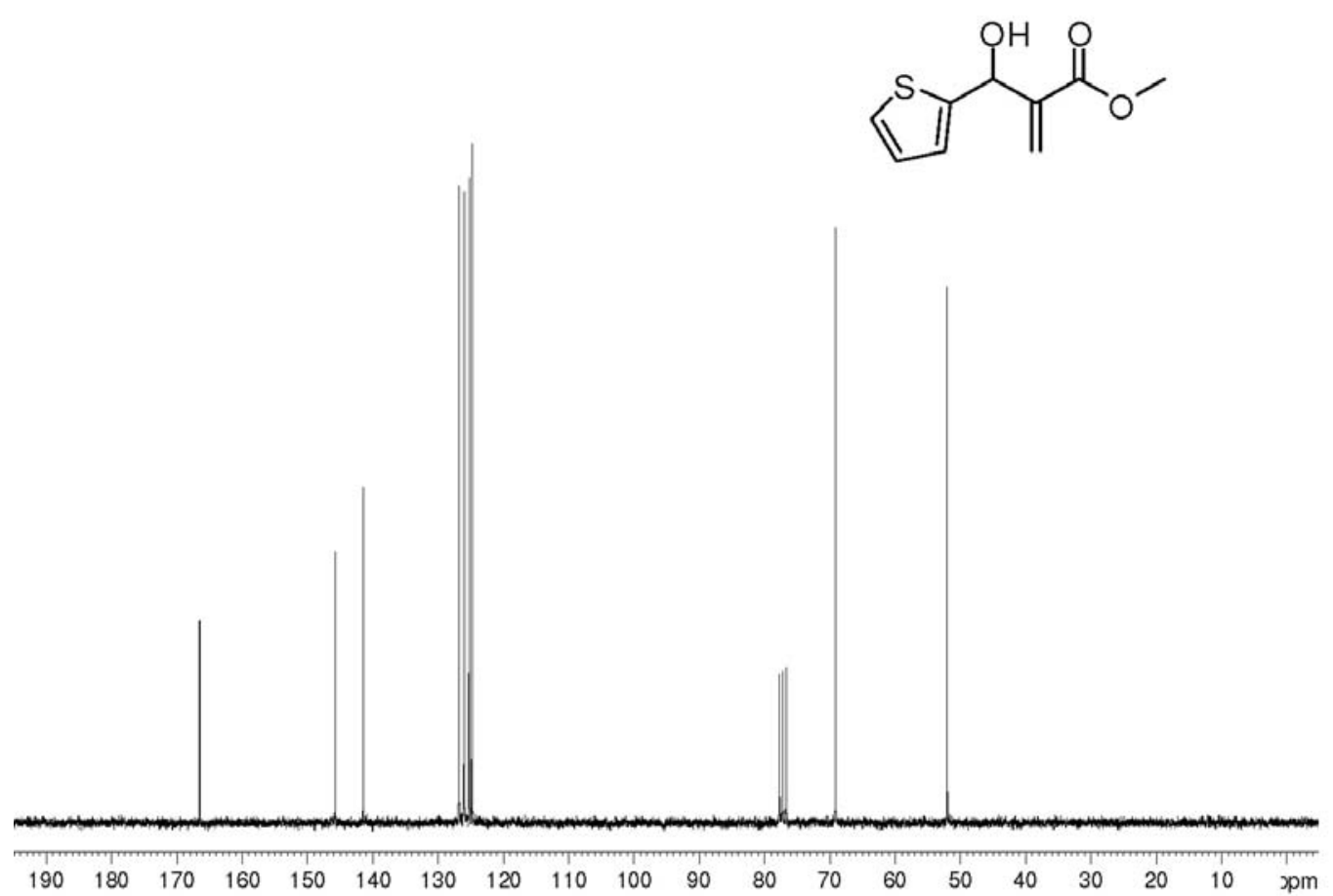

Figure S12. ${ }^{13} \mathrm{C}$ NMR $\left(\mathrm{CDCl}_{3}, 62.5 \mathrm{MHz}\right)$ of $\mathrm{MBH}$ adduct 8 .

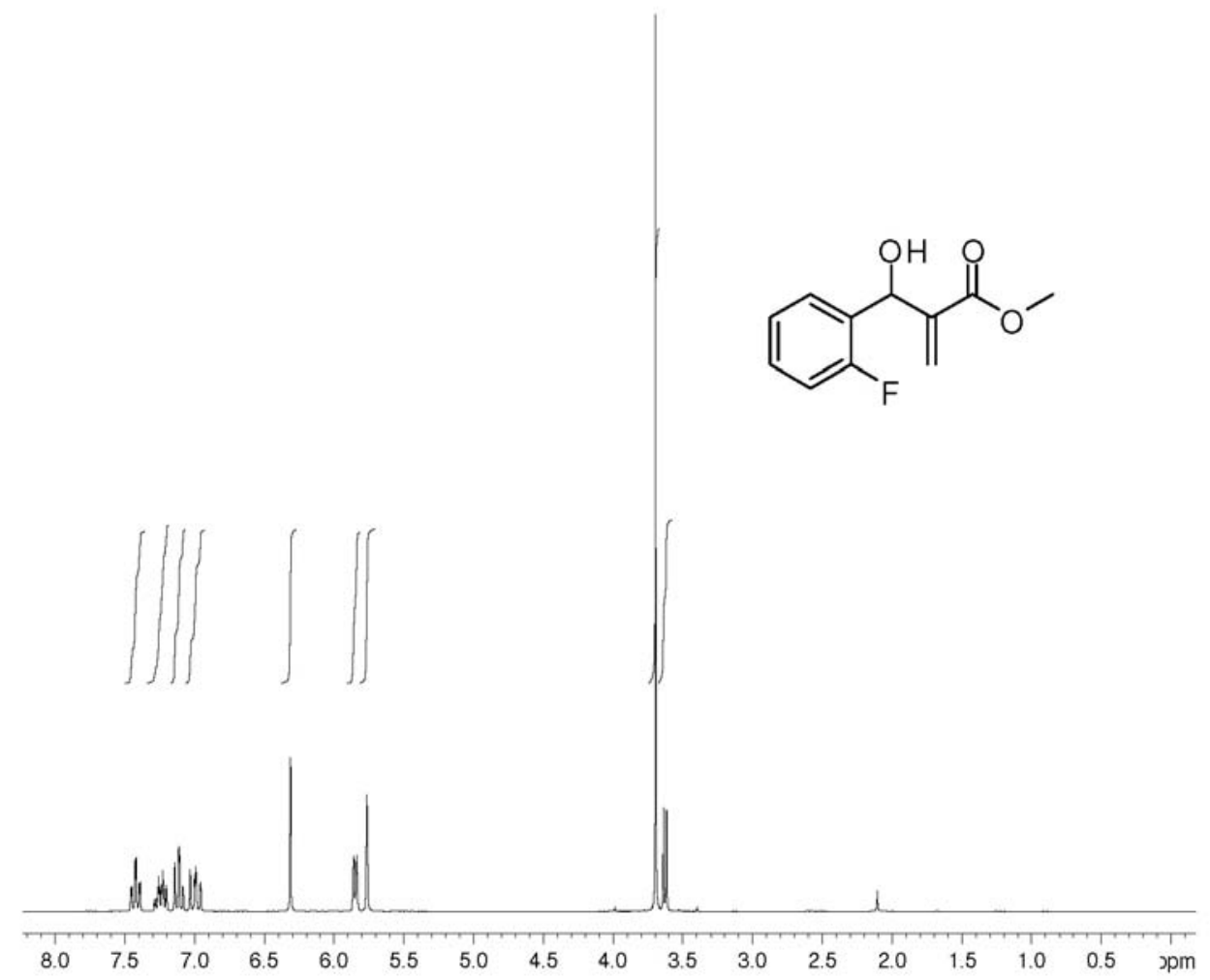

Figure S13. ${ }^{1} \mathrm{H}$ NMR $\left(\mathrm{CDCl}_{3}, 250 \mathrm{MHz}\right)$ of $\mathrm{MBH}$ adduct 9. 


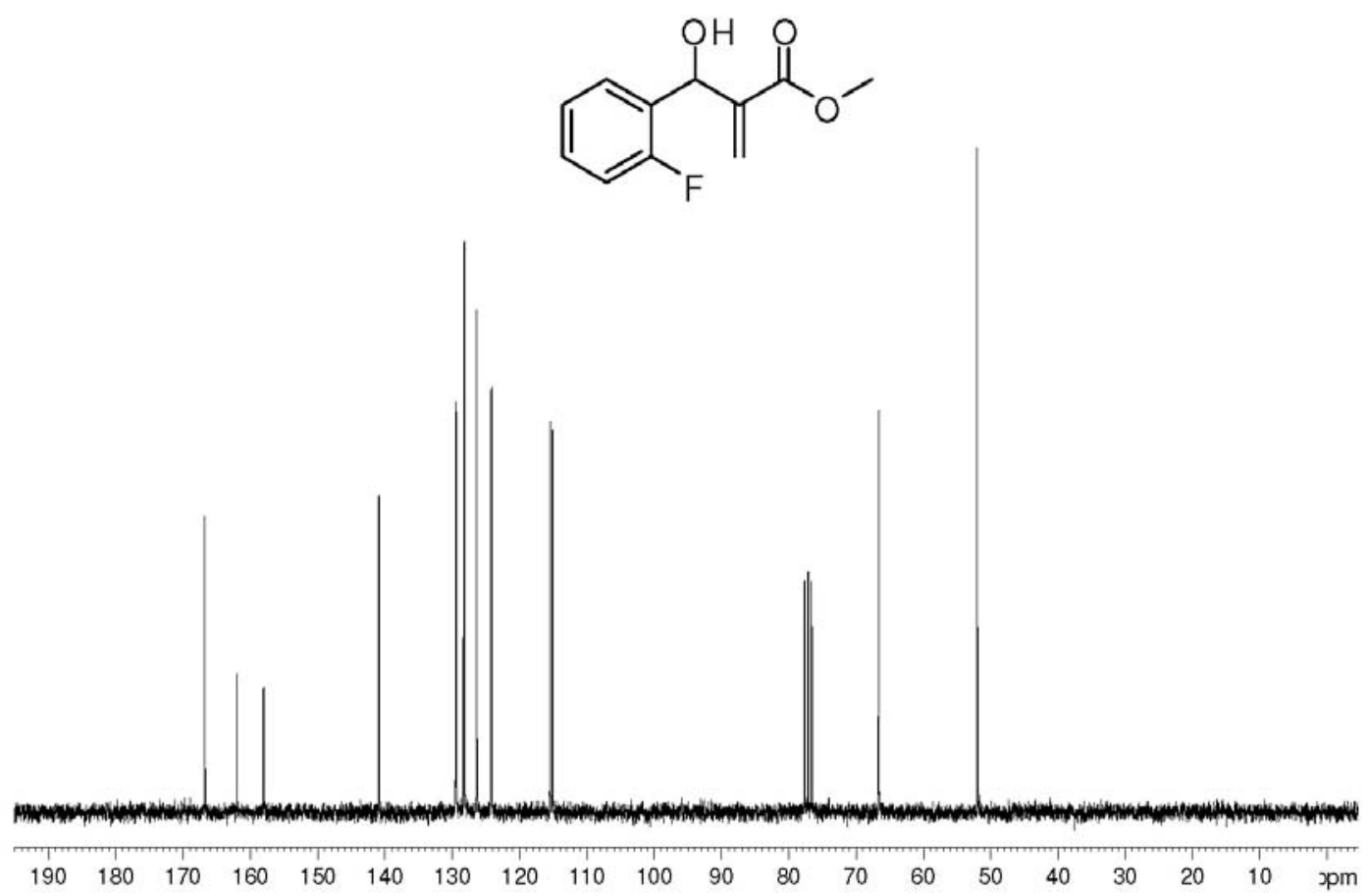

Figure S14. ${ }^{13} \mathrm{C}$ NMR $\left(\mathrm{CDCl}_{3}, 62.5 \mathrm{MHz}\right)$ of $\mathrm{MBH}$ adduct 9.

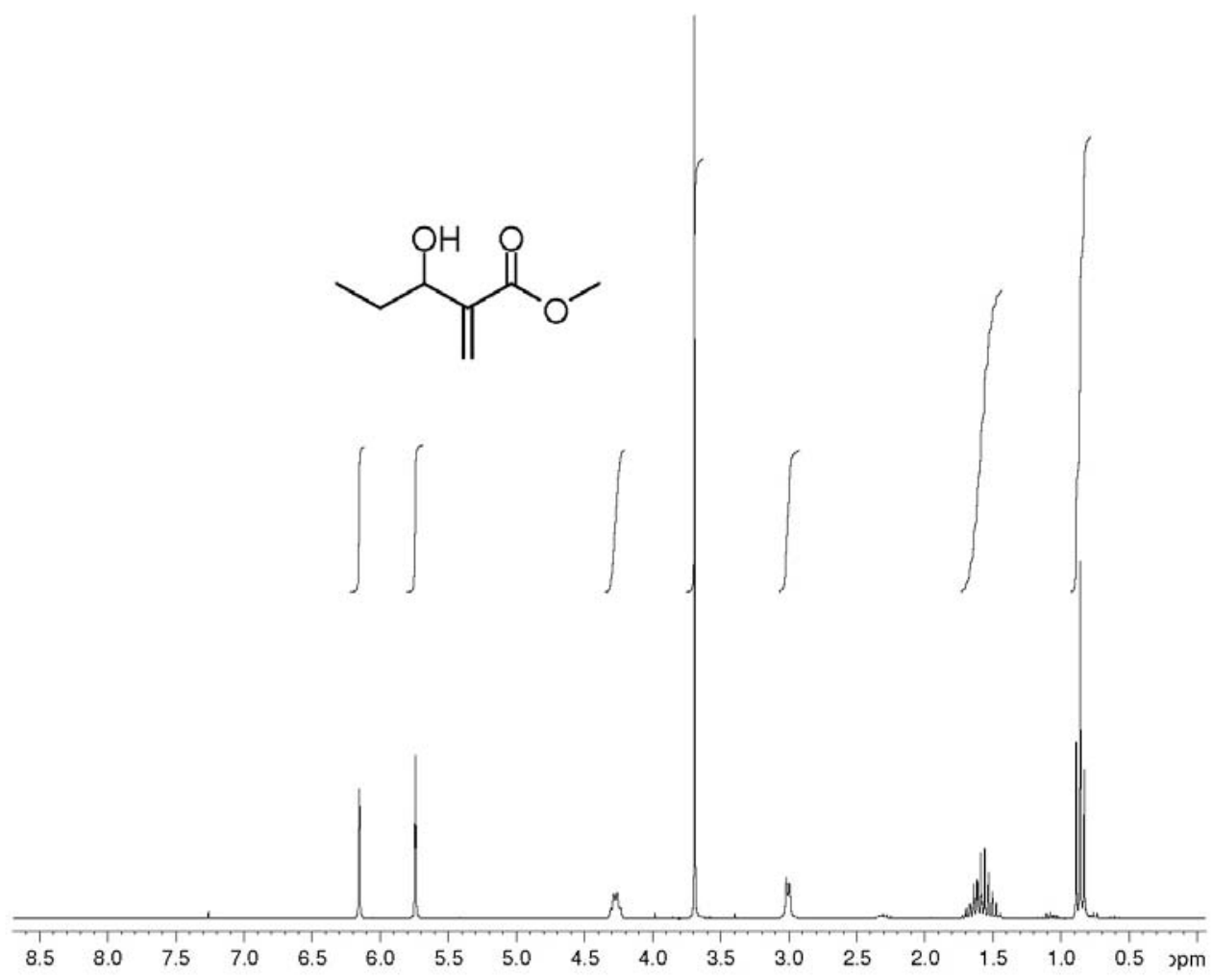

Figure S15. ${ }^{1} \mathrm{H}$ NMR $\left(\mathrm{CDCl}_{3}, 250 \mathrm{MHz}\right)$ of $\mathrm{MBH}$ adduct 10. 
<smiles>C=C(C(=O)OC)C(O)CC</smiles>

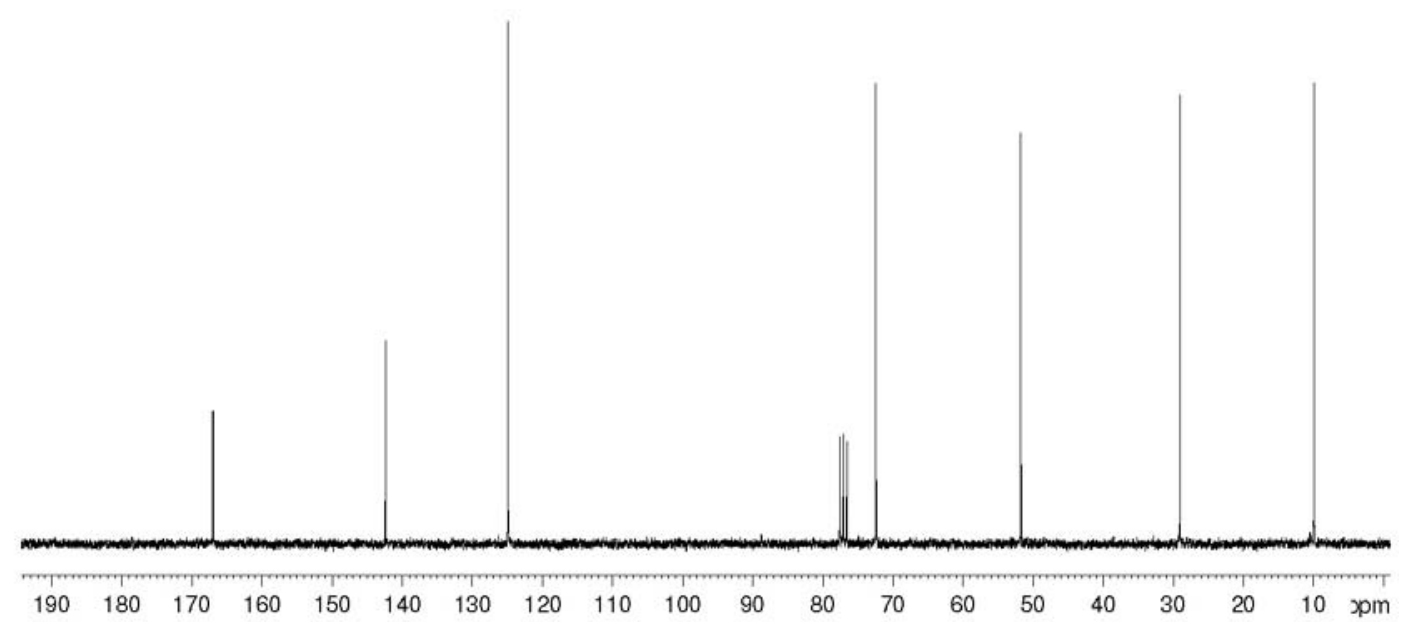

Figure S16. ${ }^{13} \mathrm{C} \mathrm{NMR}\left(\mathrm{CDCl}_{3}, 62.5 \mathrm{MHz}\right)$ of $\mathrm{MBH}$ adduct 10.

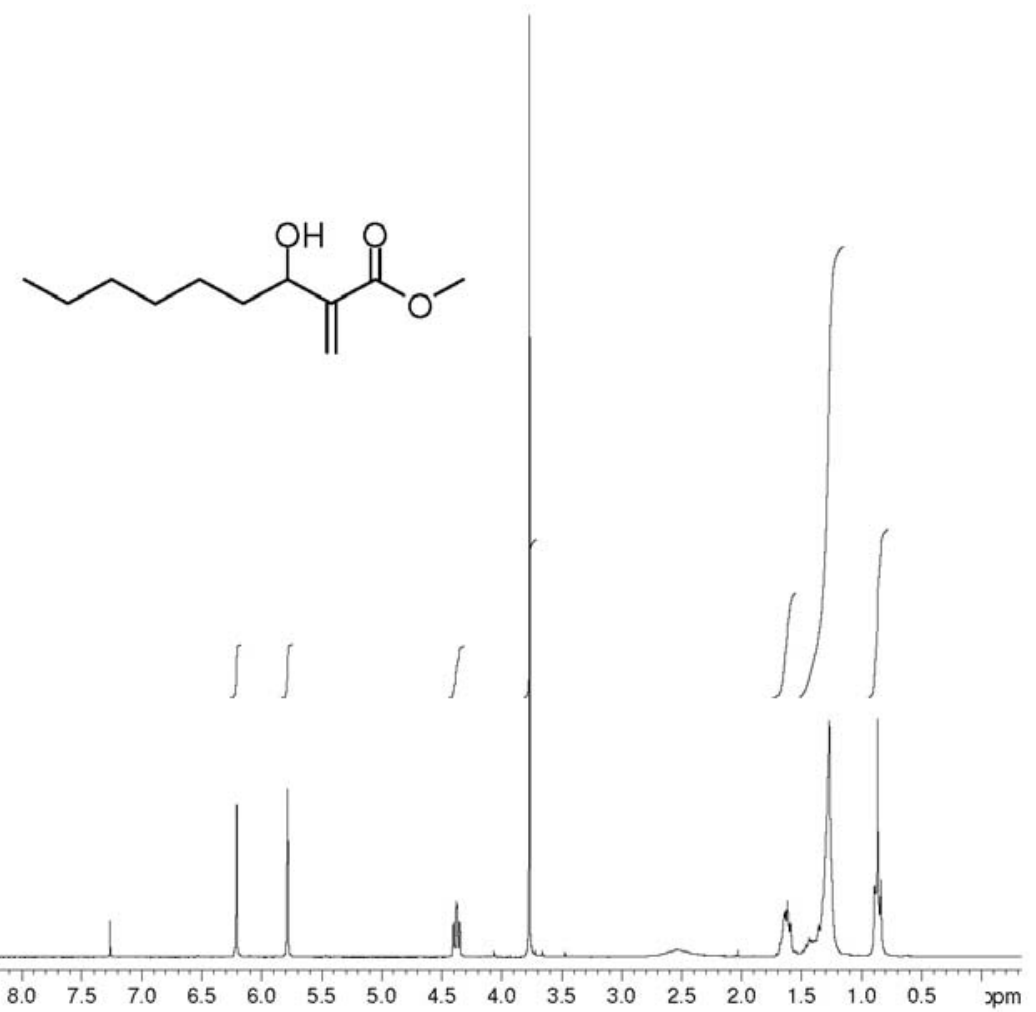

Figure S17. ${ }^{1} \mathrm{H}$ NMR $\left(\mathrm{CDCl}_{3}, 250 \mathrm{MHz}\right)$ of $\mathrm{MBH}$ adduct 11. 


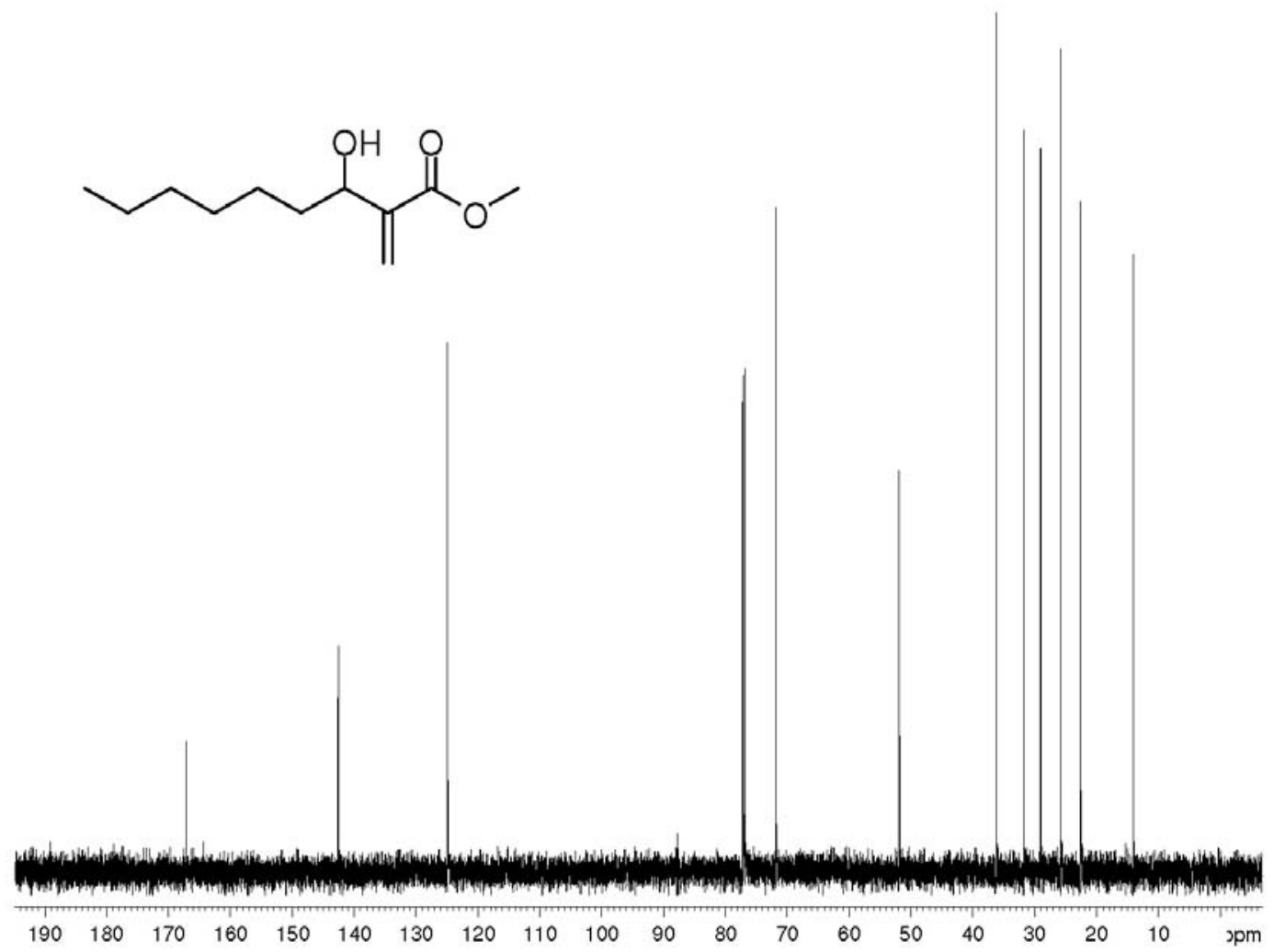

Figure S18. ${ }^{13} \mathrm{C}$ NMR $\left(\mathrm{CDCl}_{3}, 125 \mathrm{MHz}\right)$ of $\mathrm{MBH}$ adduct 11.

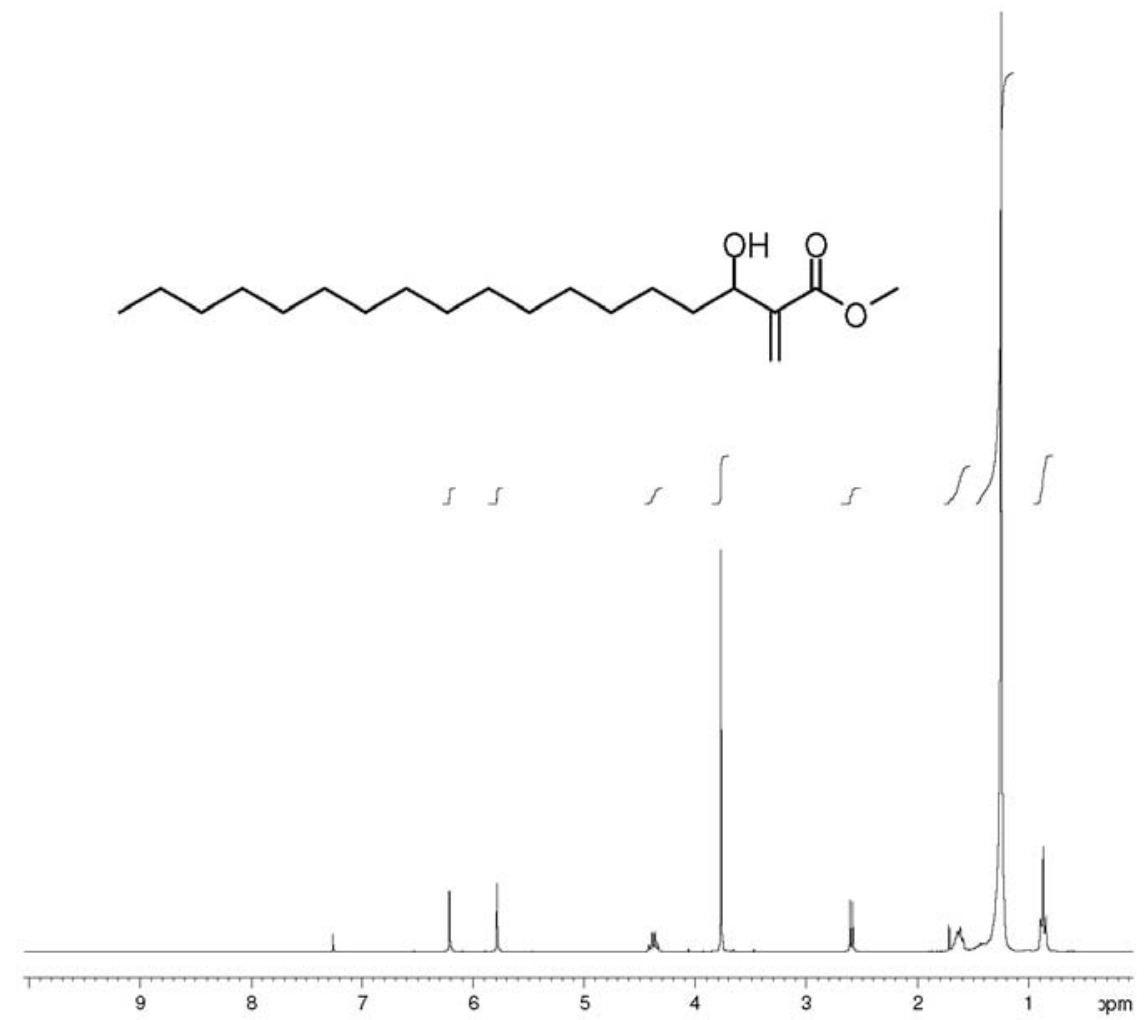

Figure S19. ${ }^{1} \mathrm{H} \mathrm{NMR}\left(\mathrm{CDCl}_{3}, 250 \mathrm{MHz}\right)$ of $\mathrm{MBH}$ adduct 12. 


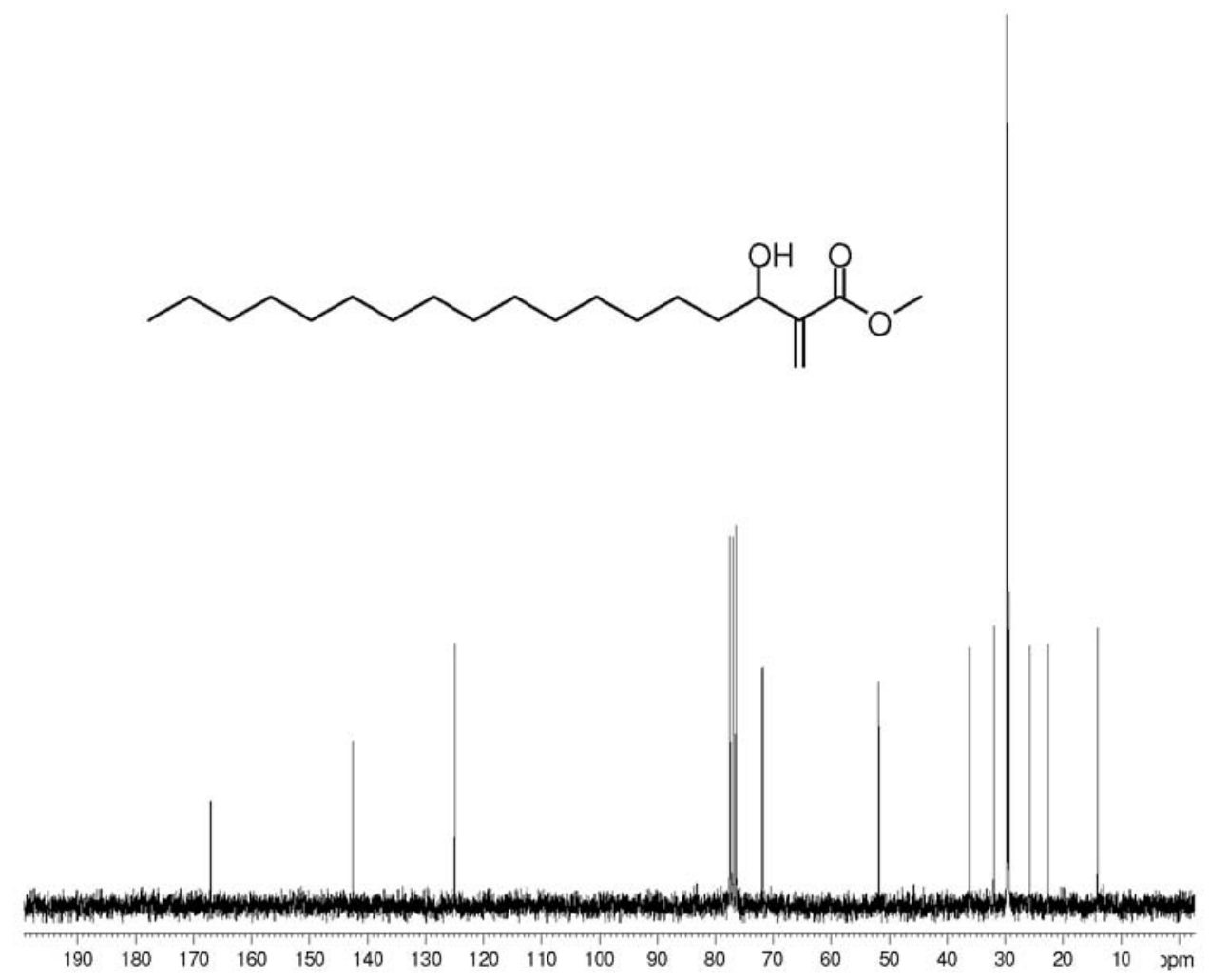

Figure S20. ${ }^{13} \mathrm{C} \mathrm{NMR}\left(\mathrm{CDCl}_{3}, 125 \mathrm{MHz}\right)$ of $\mathrm{MBH}$ adduct 12.

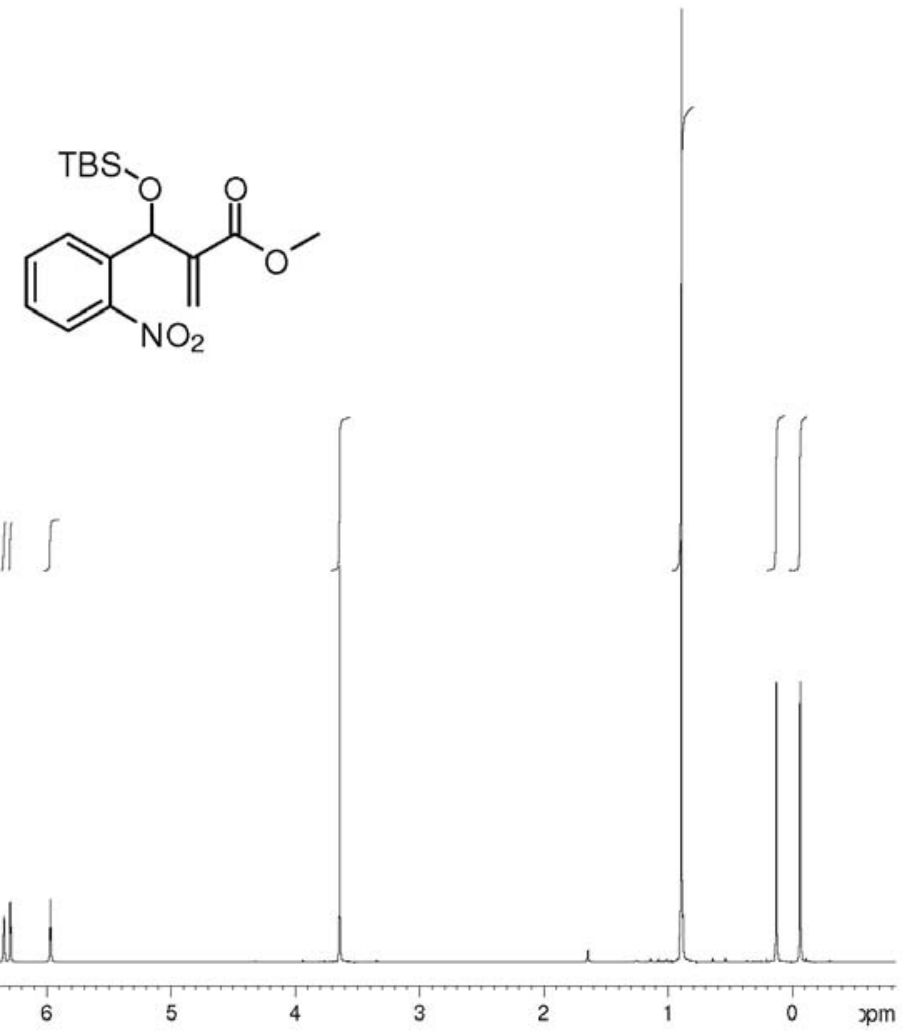

Figure S21. ${ }^{1} \mathrm{H}$ NMR $\left(\mathrm{CDCl}_{3}, 250 \mathrm{MHz}\right)$ of silylated MBH adduct 13. 


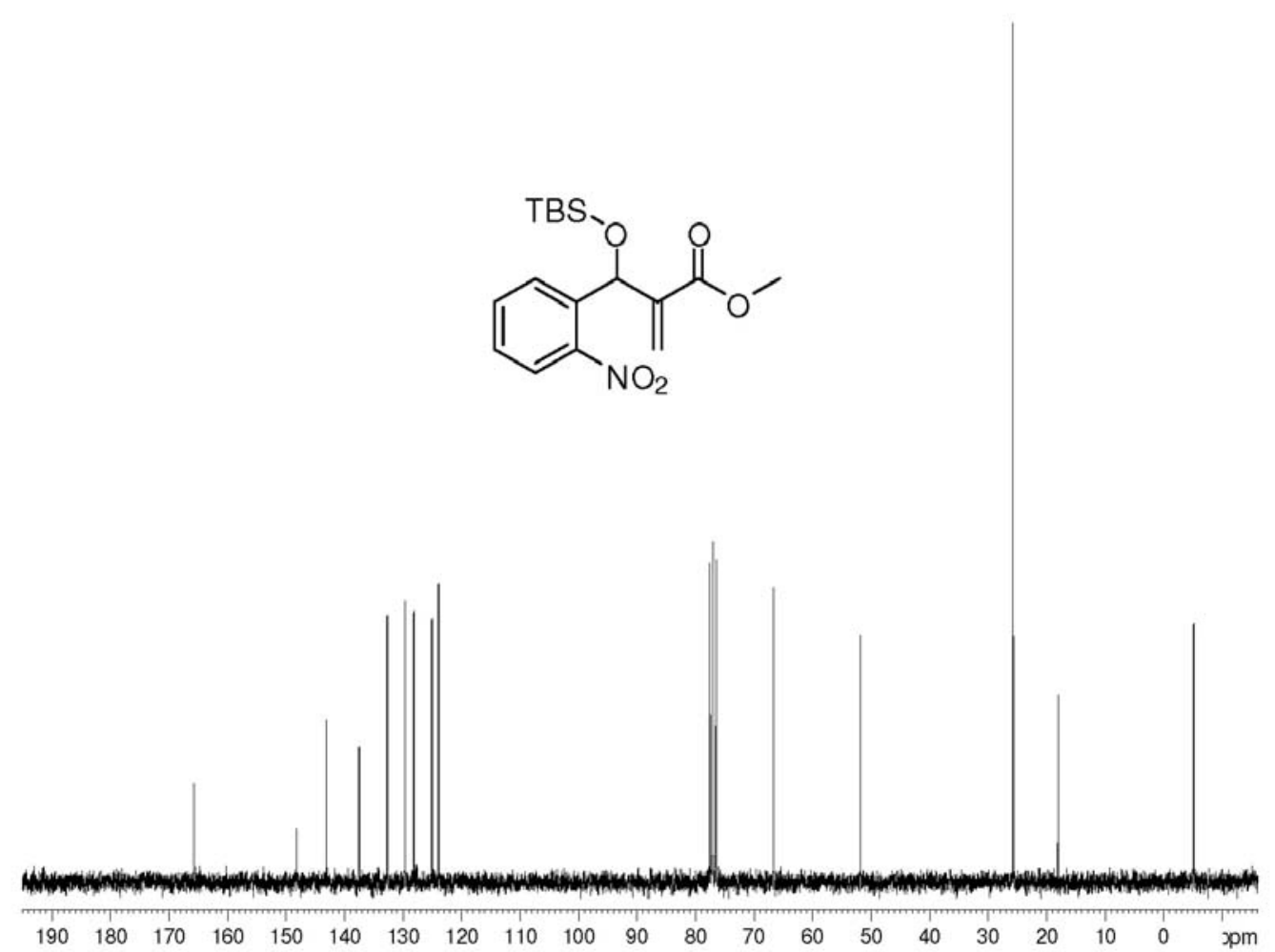

Figure S22. ${ }^{13} \mathrm{C}$ RMN $\left(\mathrm{CDCl}_{3}, 62.5 \mathrm{MHz}\right)$ of silylated $\mathrm{MBH}$ adduct $\mathbf{1 3}$.

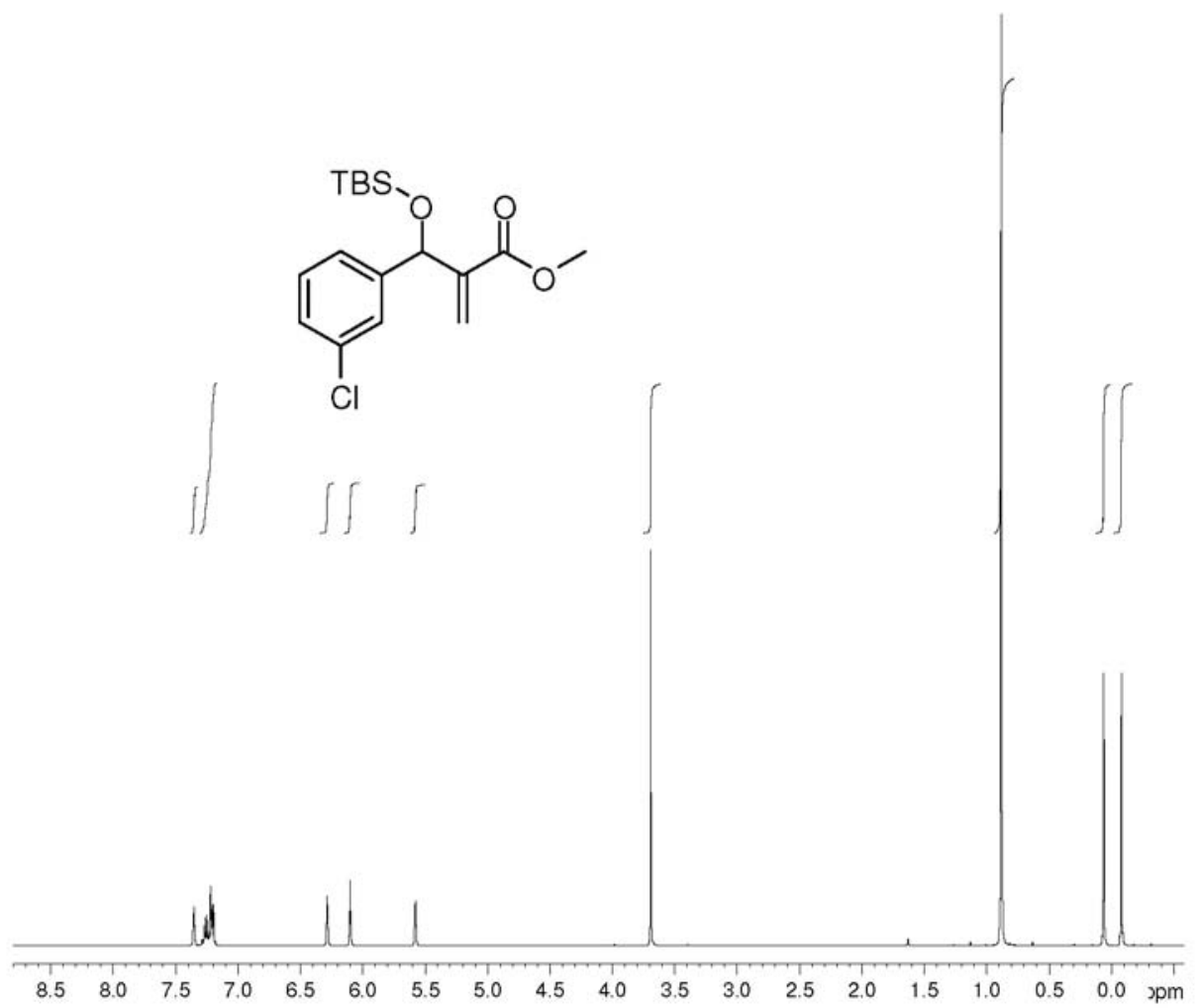

Figure S23. ${ }^{1} \mathrm{H}$ NMR $\left(\mathrm{CDCl}_{3}, 250 \mathrm{MHz}\right)$ of silylated MBH adduct 14. 


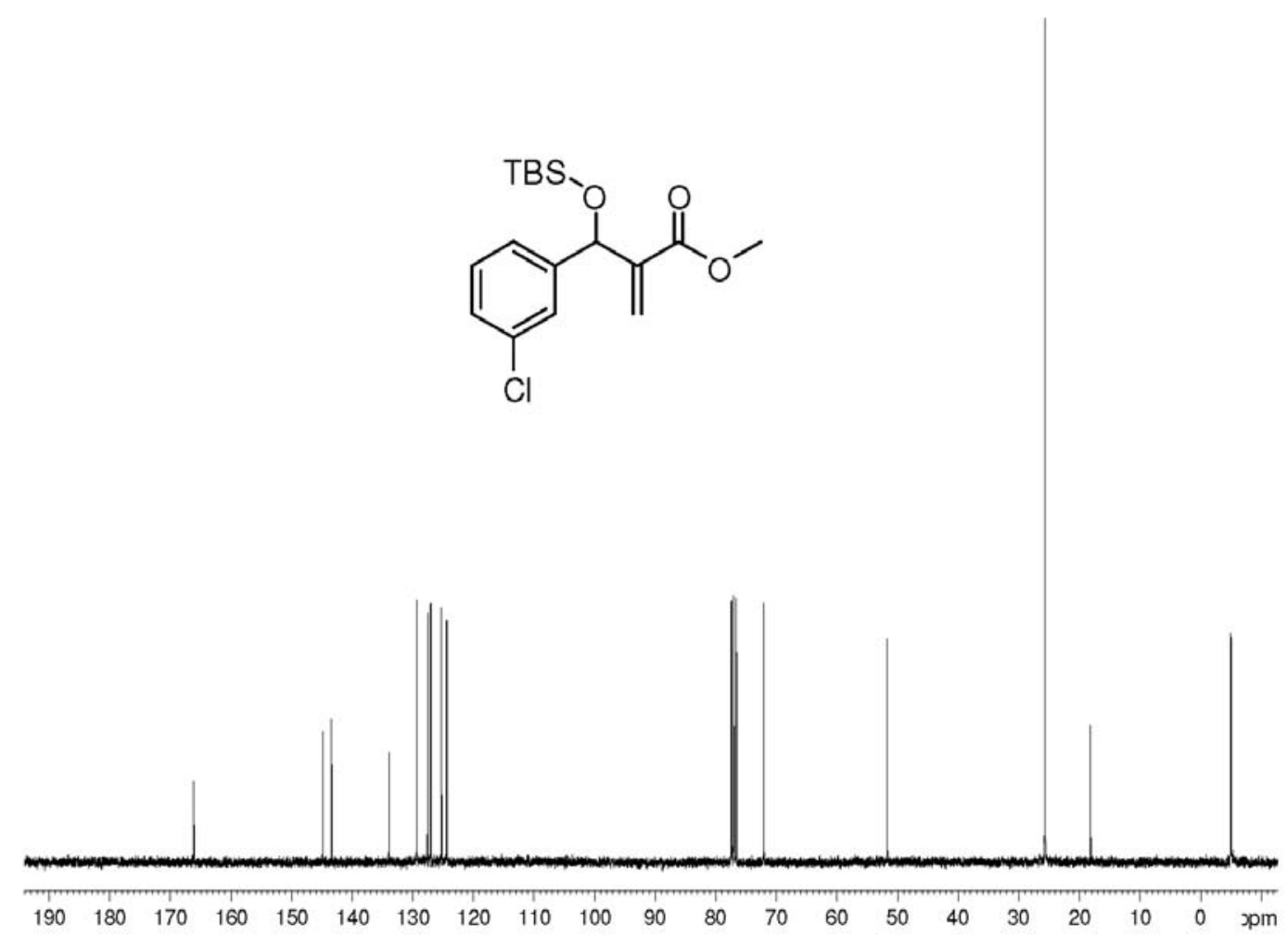

Figure S24. ${ }^{13} \mathrm{C}$ NMR $\left(\mathrm{CDCl}_{3}, 62.5 \mathrm{MHz}\right)$ of silylated $\mathrm{MBH}$ adduct 14.

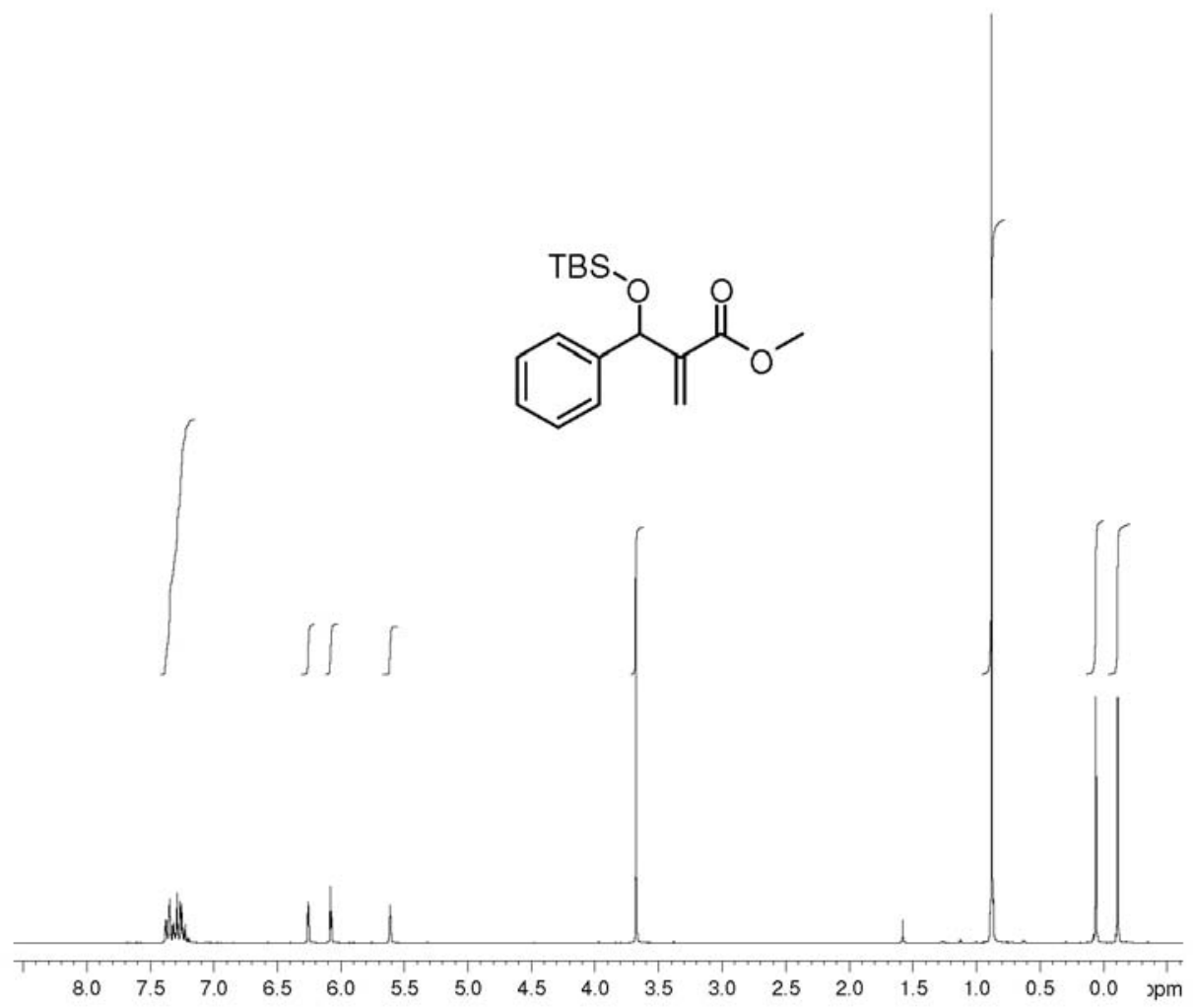

Figure S25. ${ }^{1} \mathrm{H}$ NMR $\left(\mathrm{CDCl}_{3}, 250 \mathrm{MHz}\right)$ of silylated MBH adduct 15. 


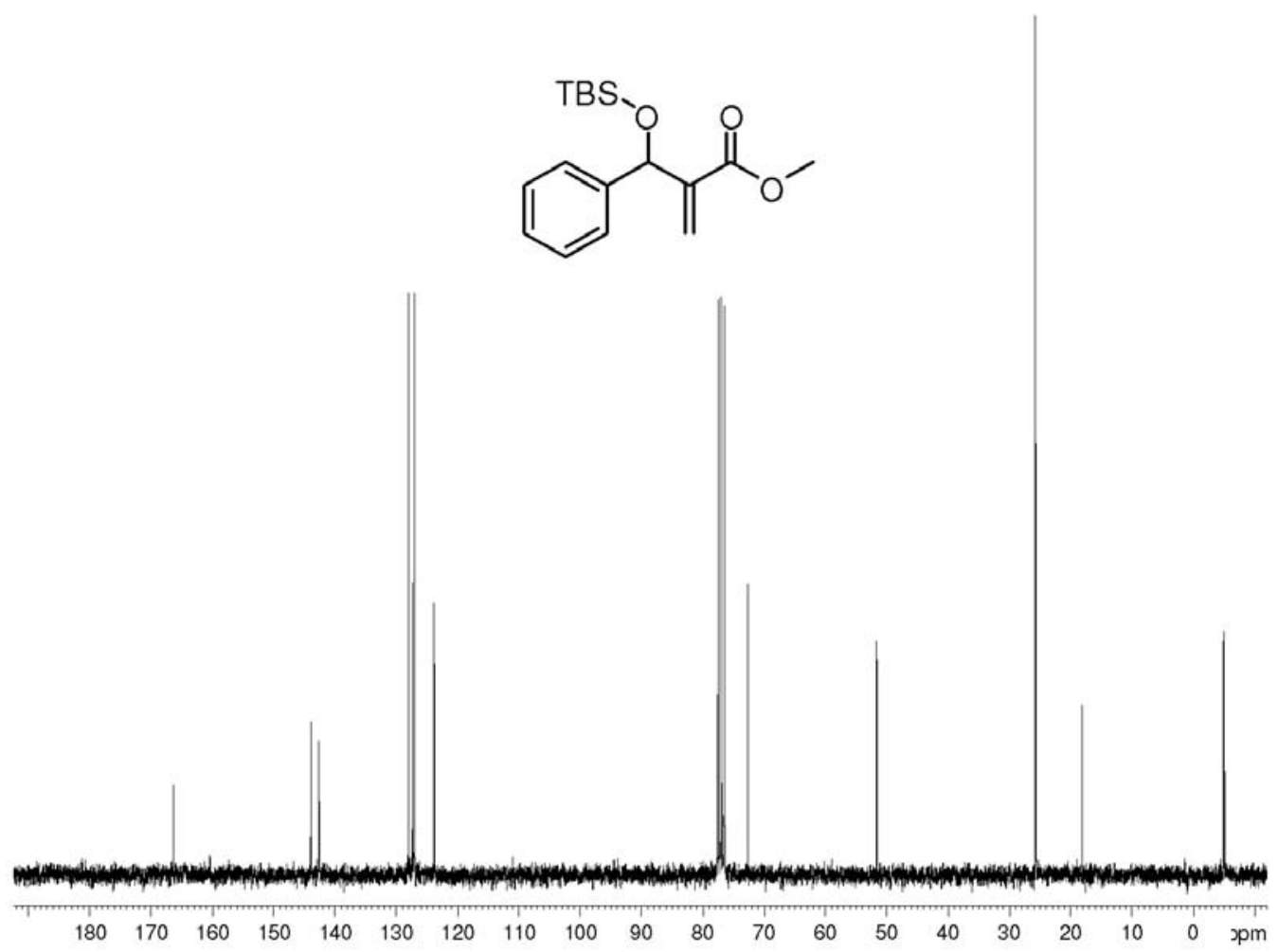

Figure S26. ${ }^{13} \mathrm{C}$ NMR $\left(\mathrm{CDCl}_{3}, 62.5 \mathrm{MHz}\right)$ of silylated $\mathrm{MBH}$ adduct 15.

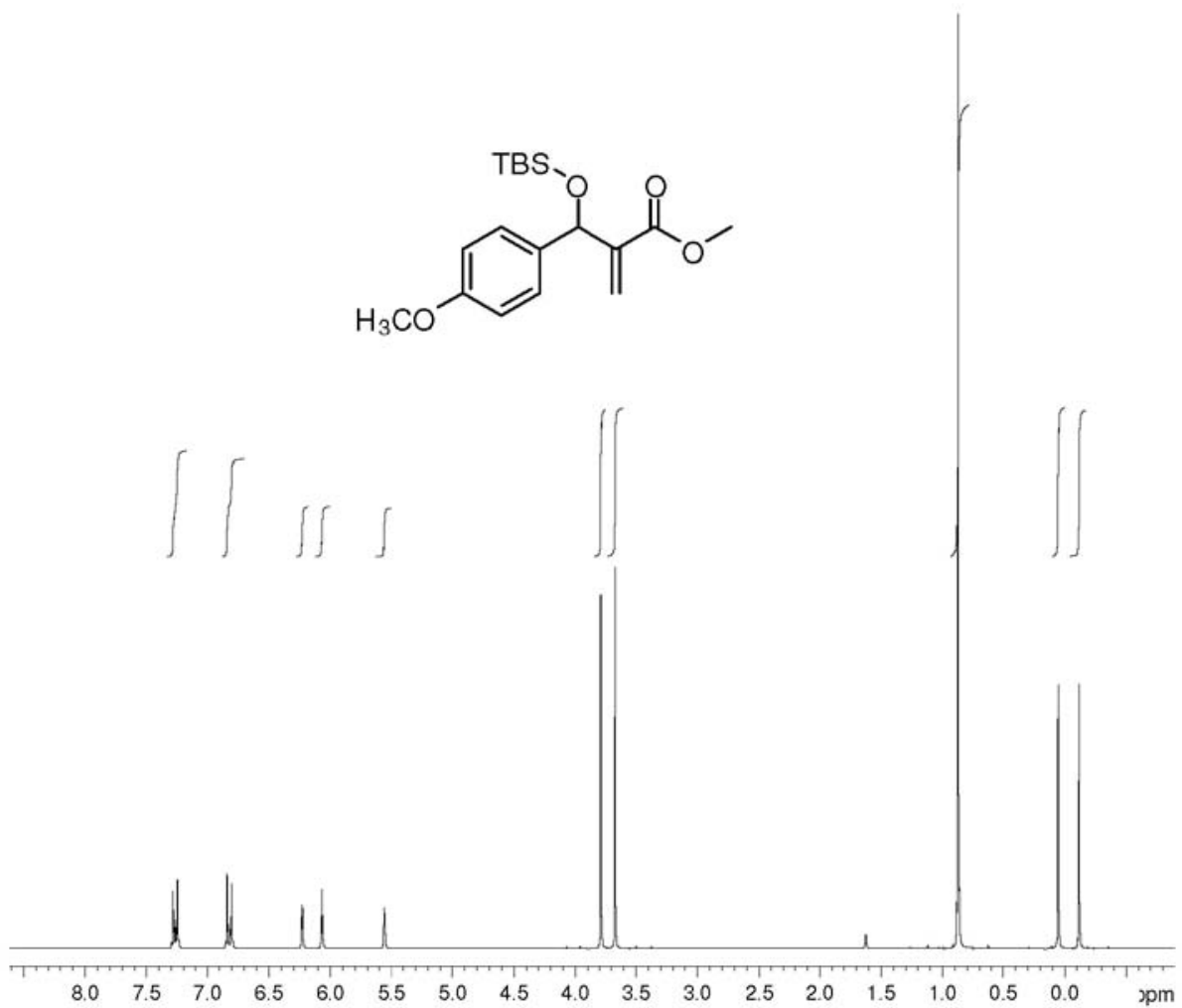

Figure S27. ${ }^{1} \mathrm{H}$ NMR $\left(\mathrm{CDCl}_{3}, 250 \mathrm{MHz}\right)$ of silylated $\mathrm{MBH}$ adduct $\mathbf{1 6}$ 


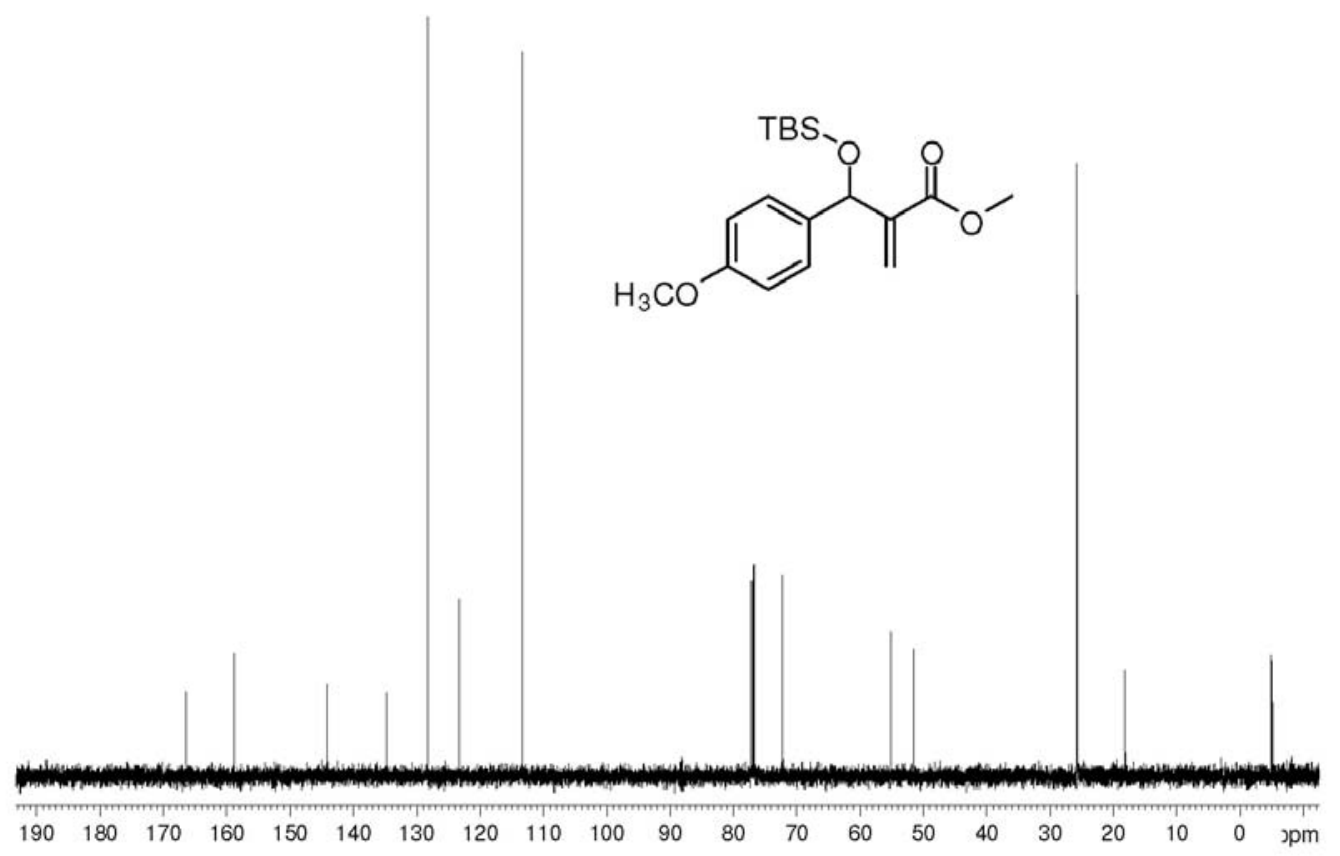

Figure S28. ${ }^{13} \mathrm{C}$ NMR $\left(\mathrm{CDCl}_{3}, 125 \mathrm{MHz}\right)$ of silylated $\mathrm{MBH}$ adduct 16.

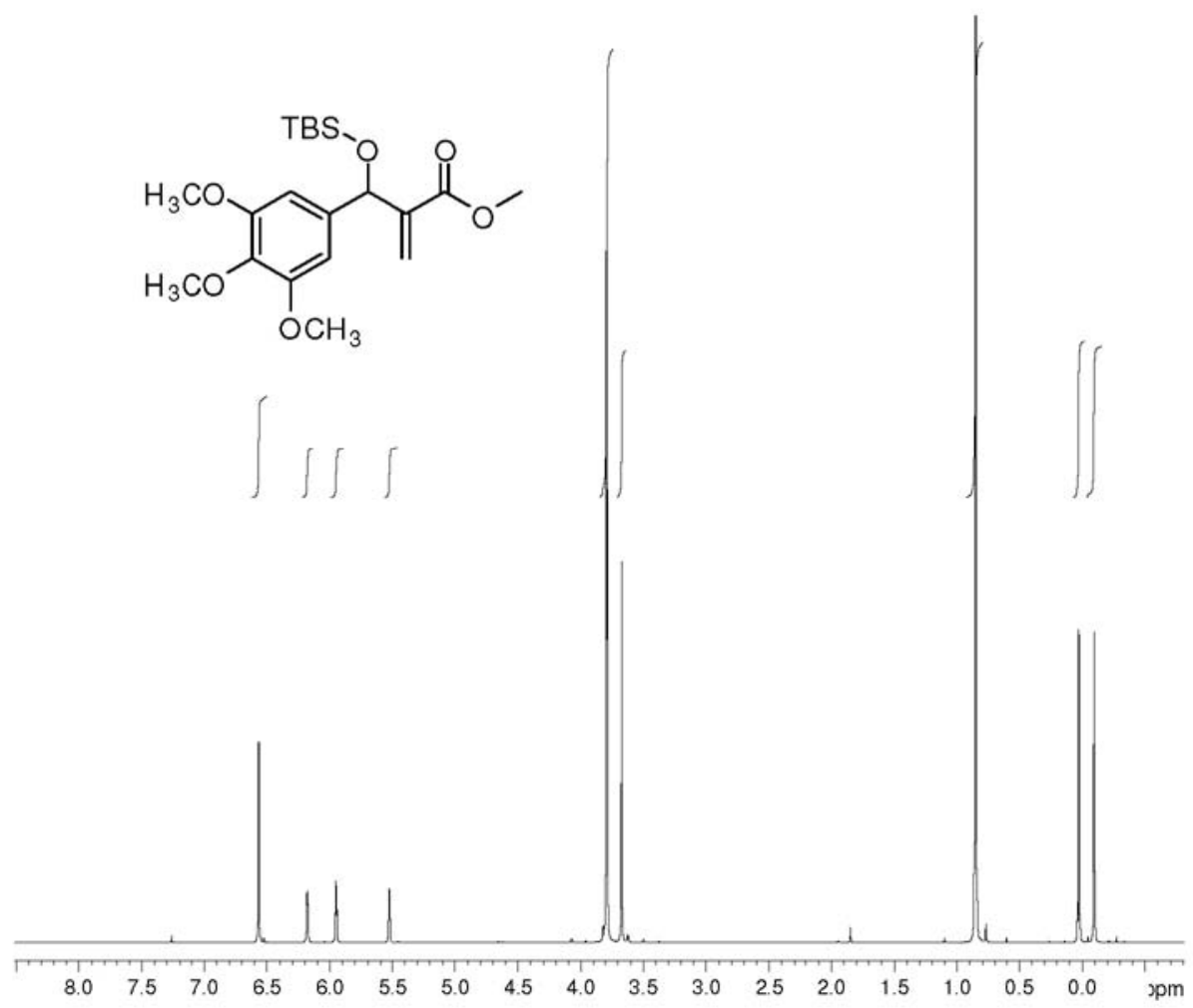

Figure S29. ${ }^{1} \mathrm{H}$ NMR $\left(\mathrm{CDCl}_{3}, 250 \mathrm{MHz}\right)$ of silylated MBH adduct 17. 


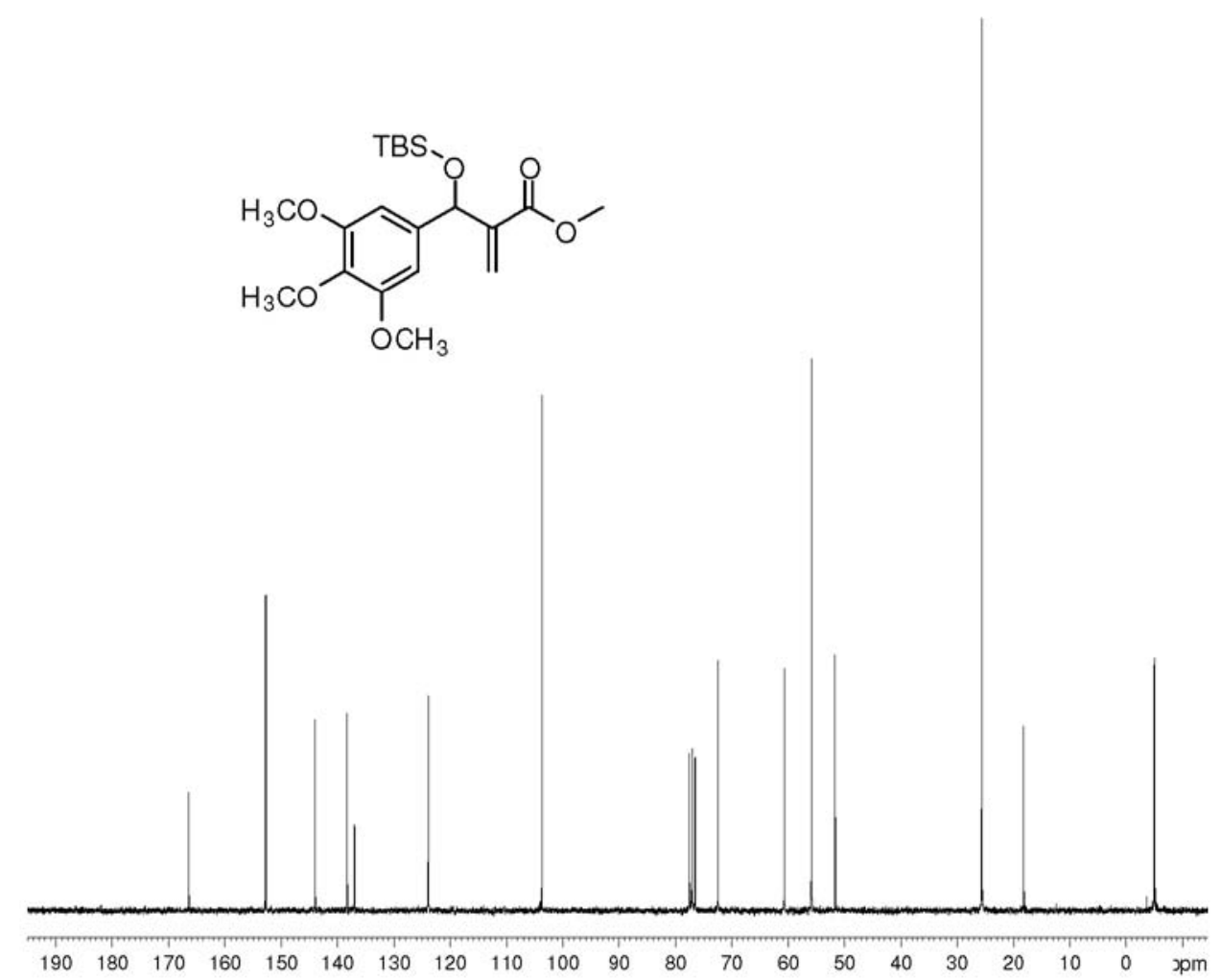

Figure S30. ${ }^{13} \mathrm{C} \mathrm{NMR}\left(\mathrm{CDCl}_{3}, 62.5 \mathrm{MHz}\right)$ of silylated $\mathrm{MBH}$ adduct 17.

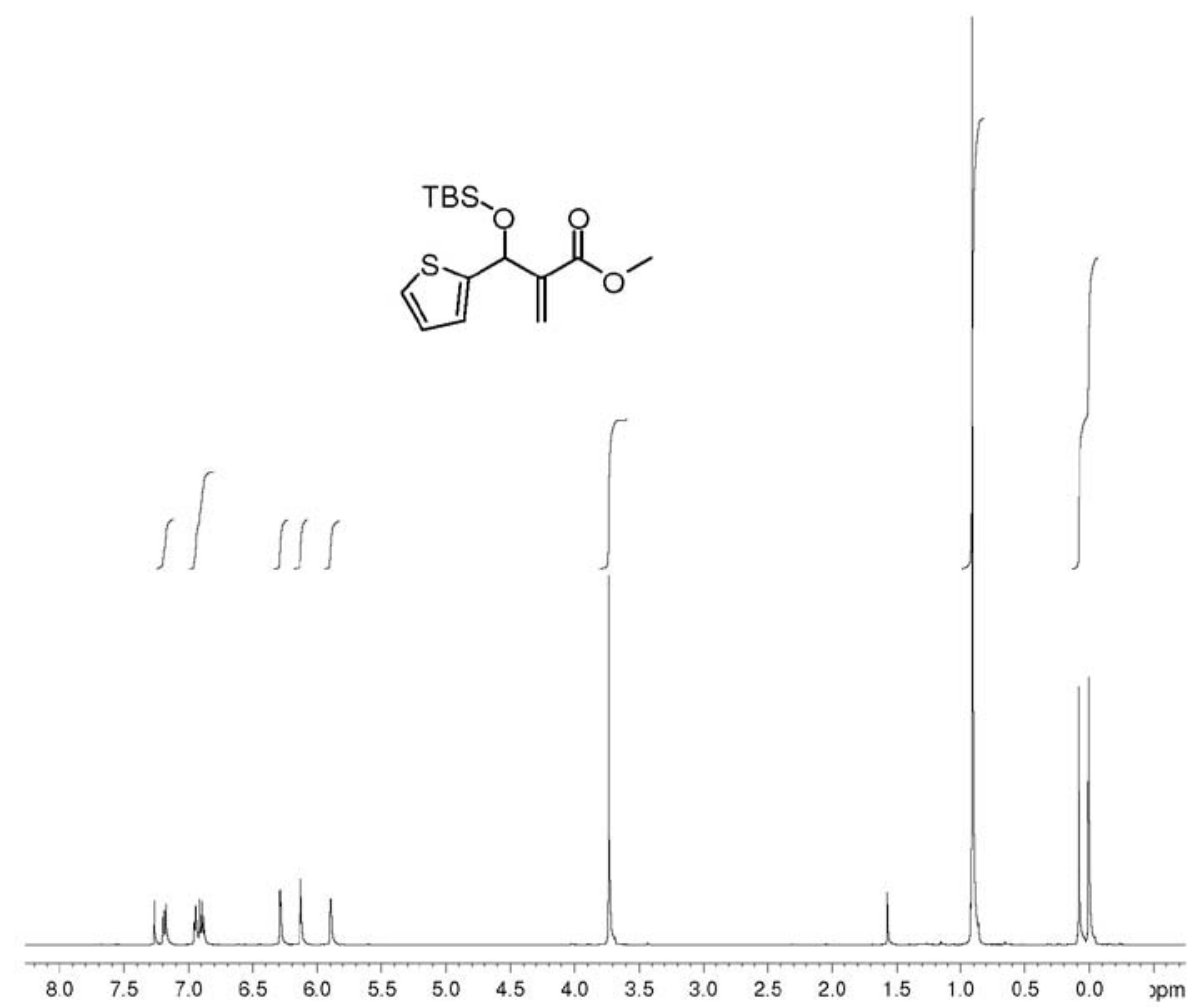

Figure S31. ${ }^{1} \mathrm{H}$ NMR $\left(\mathrm{CDCl}_{3}, 250 \mathrm{MHz}\right)$ of silylated $\mathrm{MBH}$ adduct 18. 


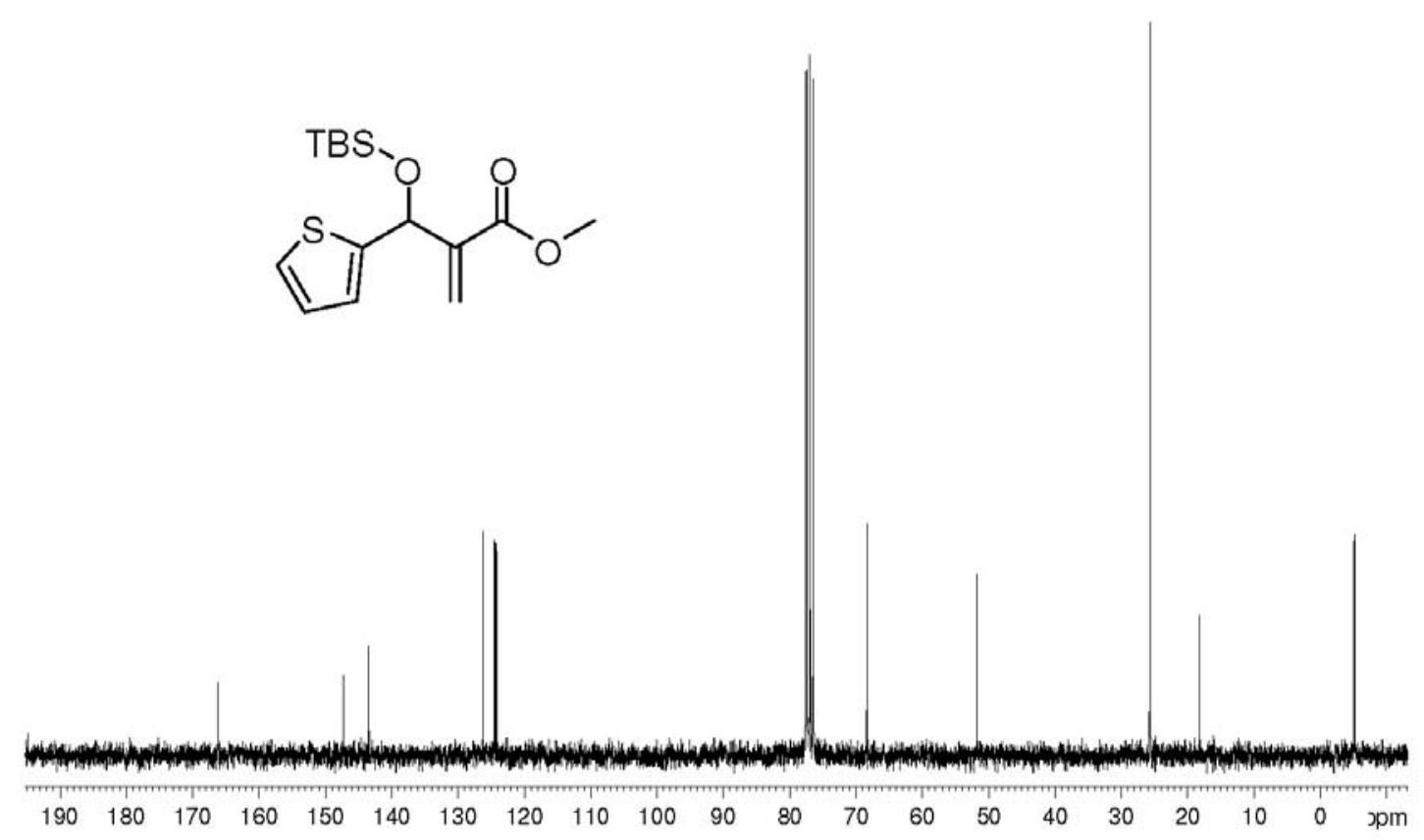

Figure S32. ${ }^{13} \mathrm{C}$ NMR $\left(\mathrm{CDCl}_{3}, 62.5 \mathrm{MHz}\right)$ of silylated $\mathrm{MBH}$ adduct 18.

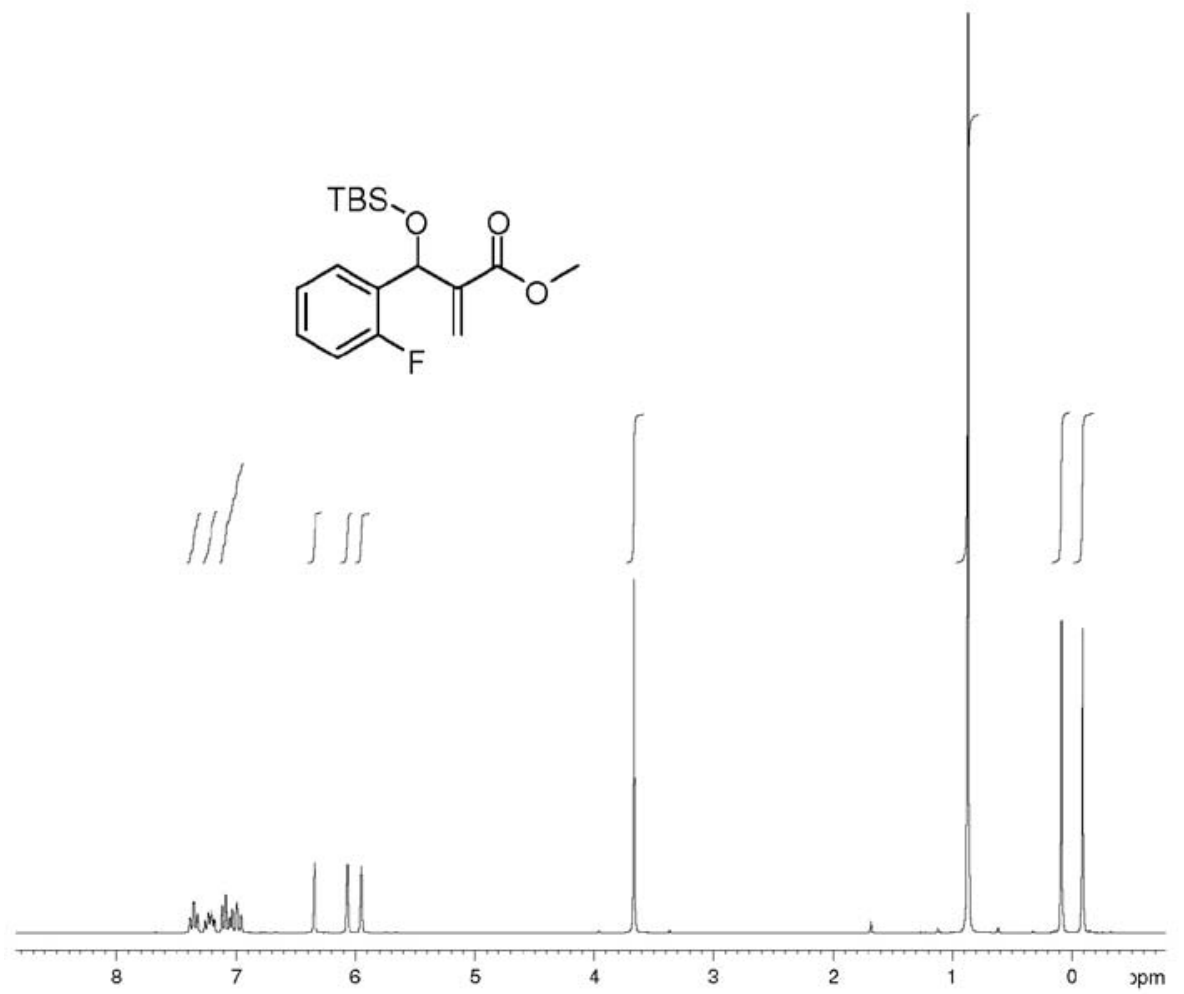

Figure S33. ${ }^{1} \mathrm{H}$ NMR $\left(\mathrm{CDCl}_{3}, 250 \mathrm{MHz}\right)$ of silylated MBH adduct 19. 


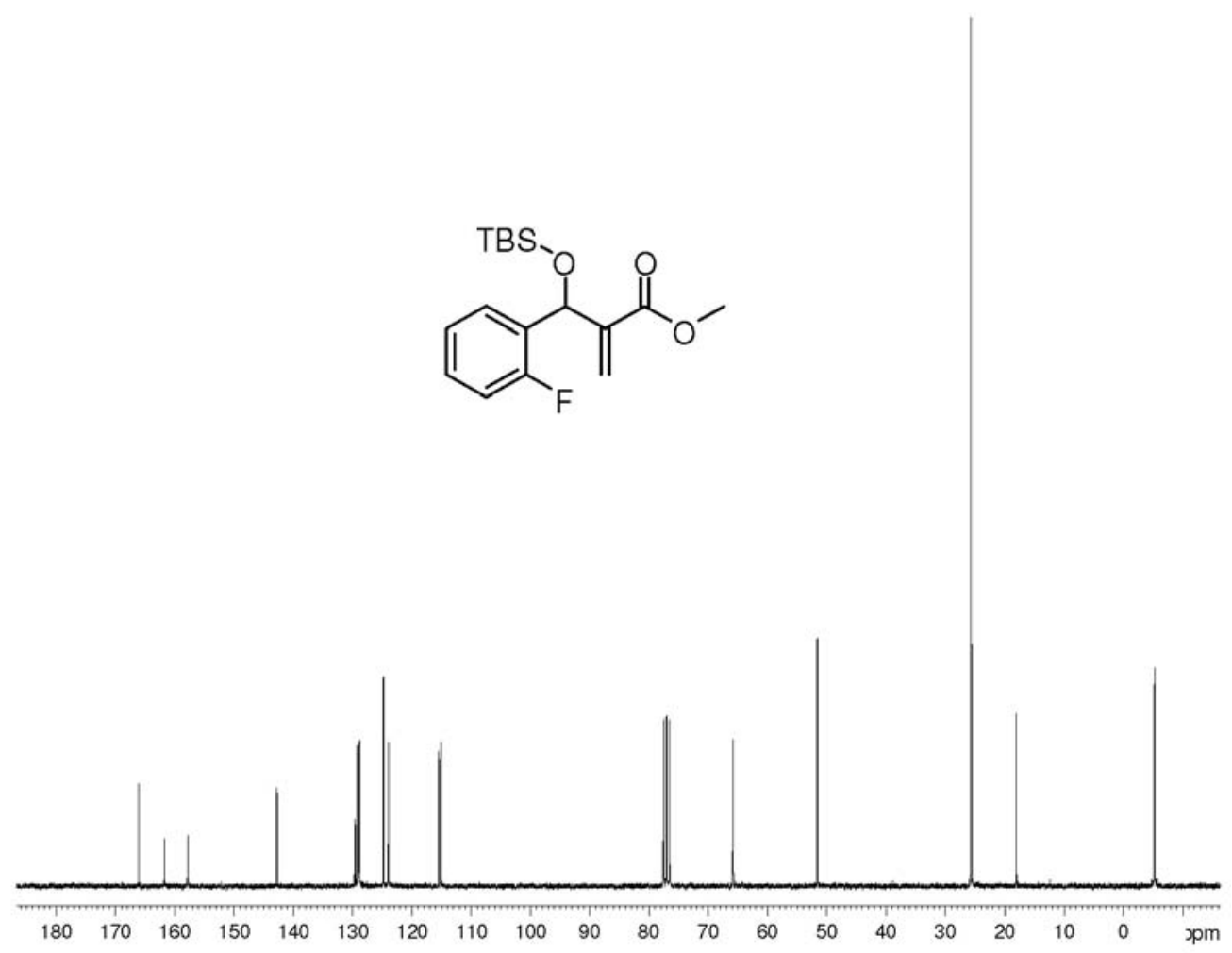

Figure S34. ${ }^{13} \mathrm{C}$ NMR $\left(\mathrm{CDCl}_{3}, 62.5 \mathrm{MHz}\right)$ of silylated $\mathrm{MBH}$ adduct 19.

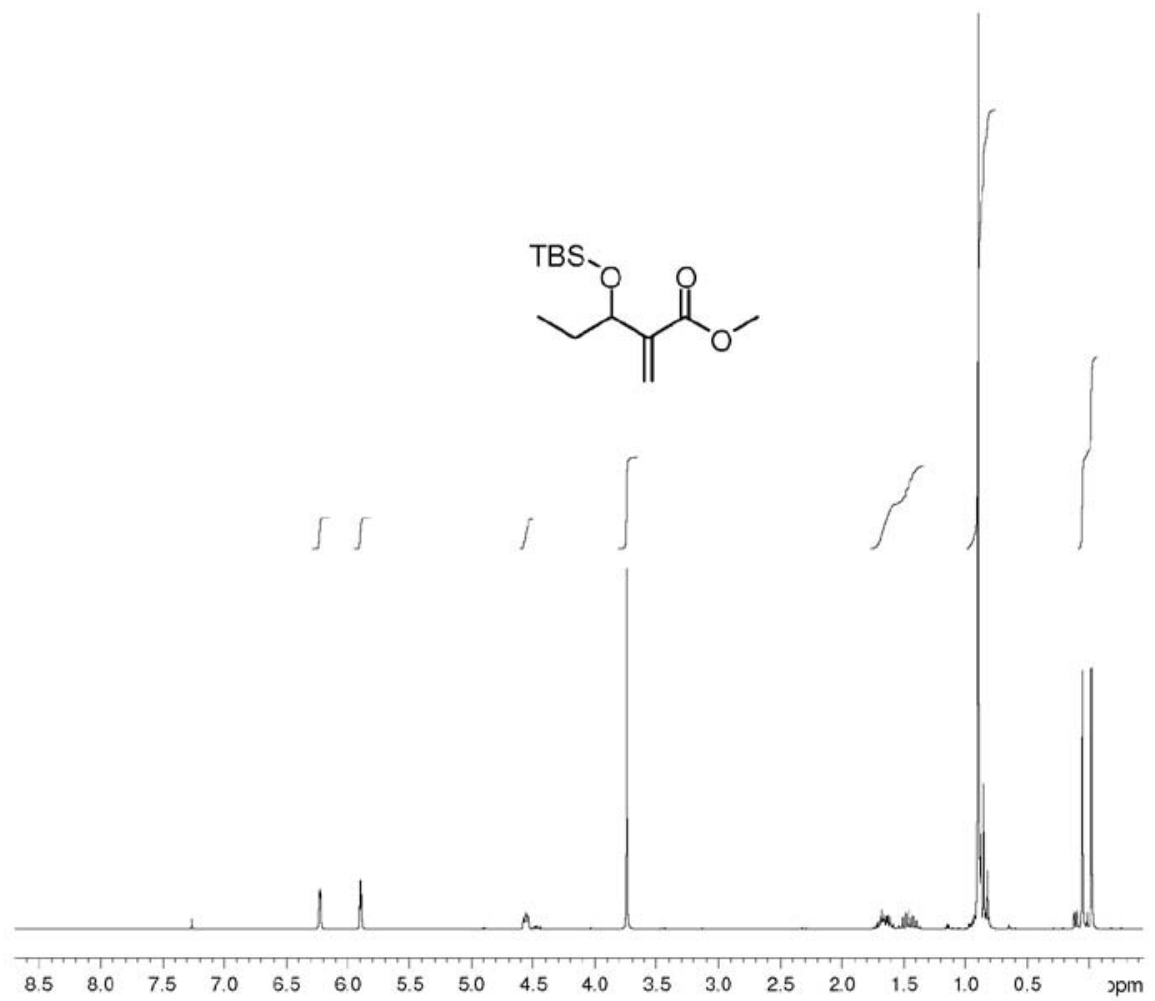

Figure S35. ${ }^{1} \mathrm{H}$ NMR $\left(\mathrm{CDCl}_{3}, 250 \mathrm{MHz}\right)$ of silylated $\mathrm{MBH}$ adduct 20. 


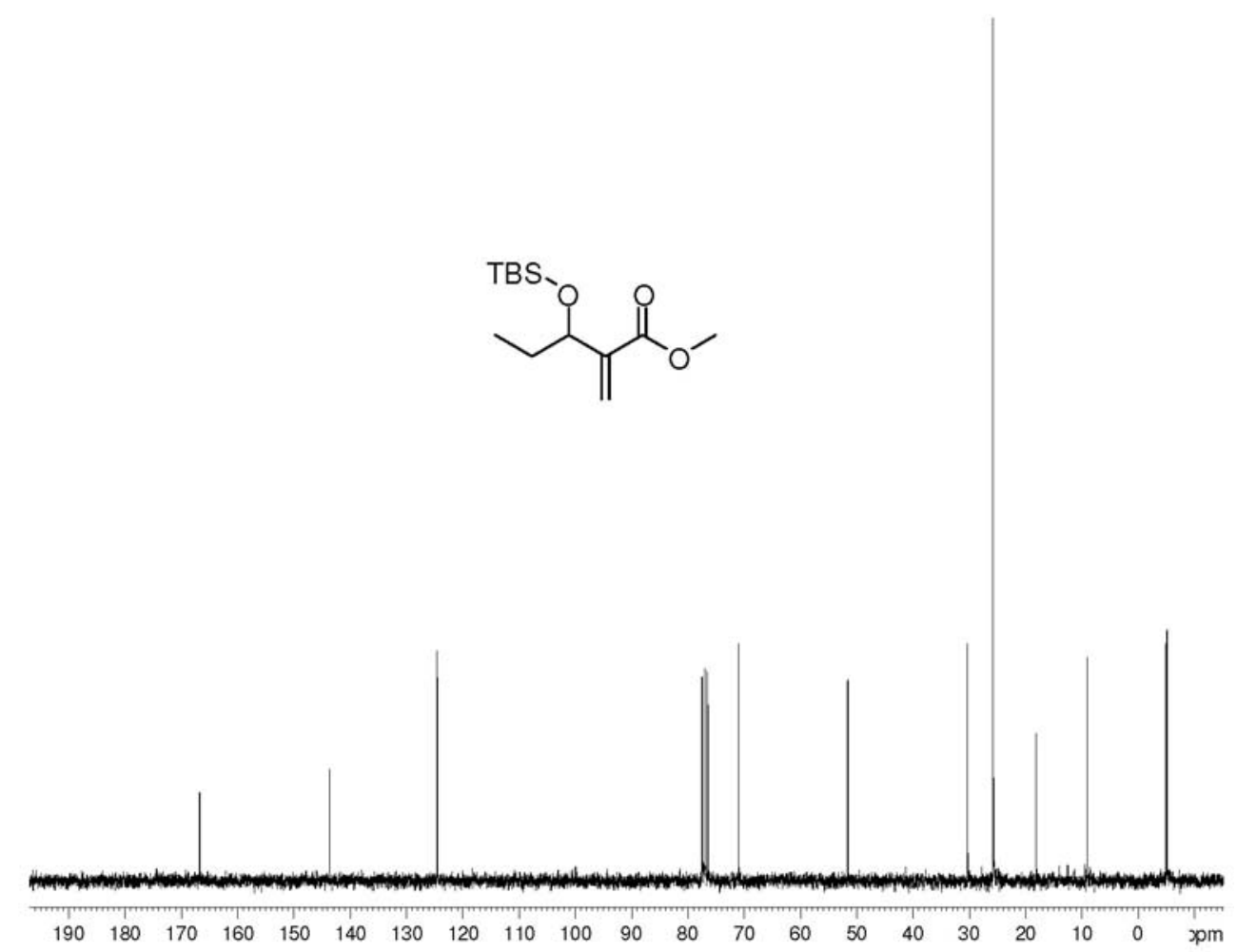

Figure S36. ${ }^{13} \mathrm{C}$ NMR $\left(\mathrm{CDCl}_{3}, 62.5 \mathrm{MHz}\right)$ of silylated $\mathrm{MBH}$ adduct 20.

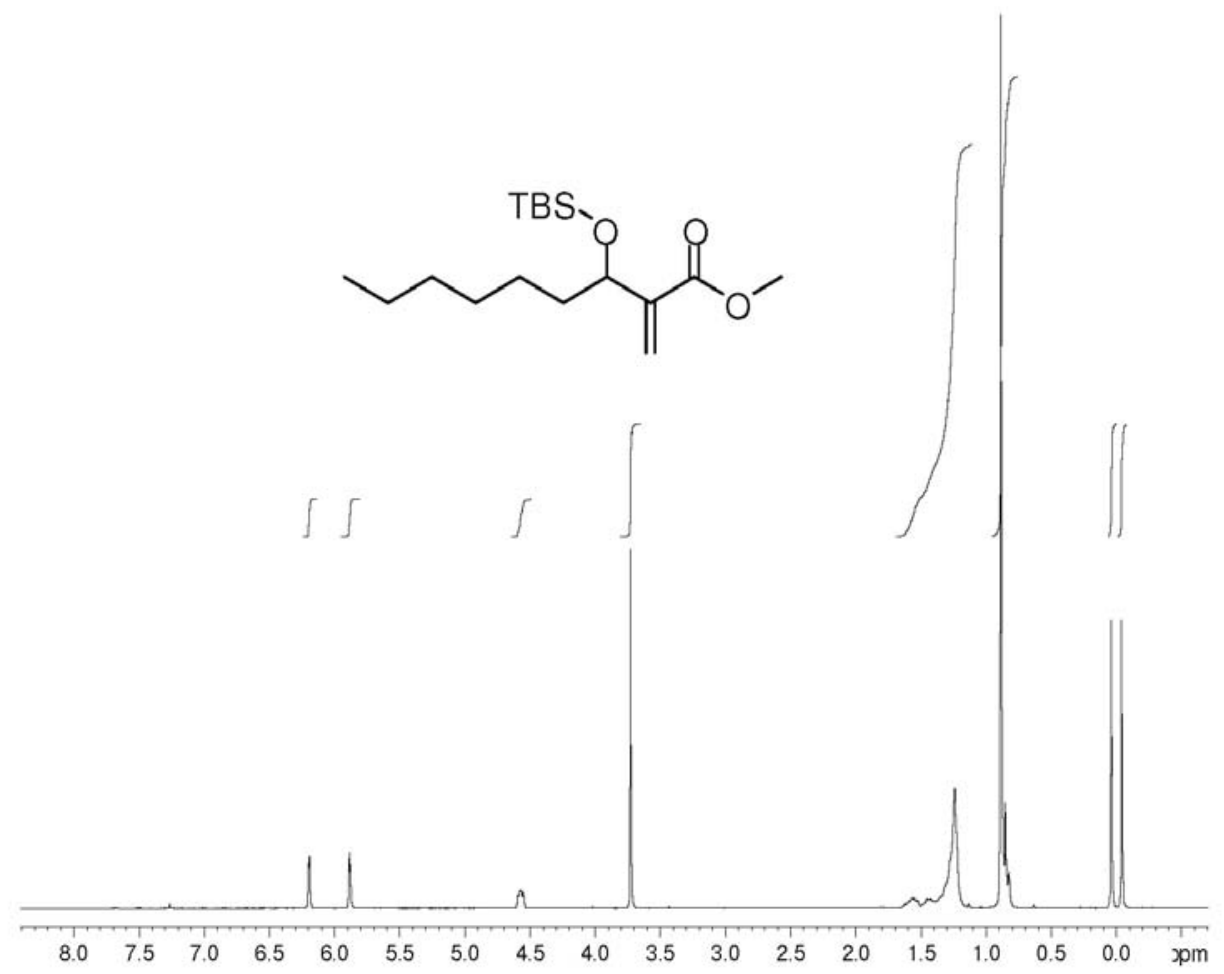

Figure S37. ${ }^{1} \mathrm{H}$ NMR $\left(\mathrm{CDCl}_{3}, 250 \mathrm{MHz}\right)$ of silylated MBH adduct 21. 


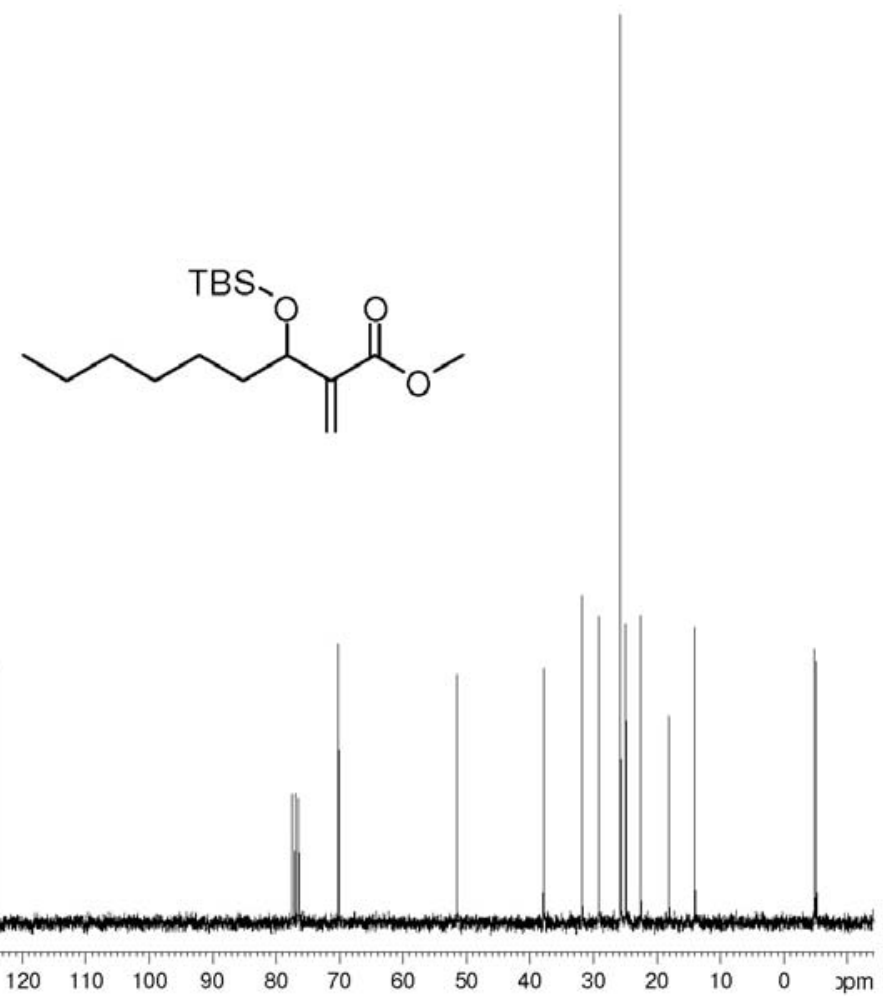

Figure S38. ${ }^{13} \mathrm{C}$ NMR $\left(\mathrm{CDCl}_{3}, 62.5 \mathrm{MHz}\right)$ of silylated $\mathrm{MBH}$ adduct 21.

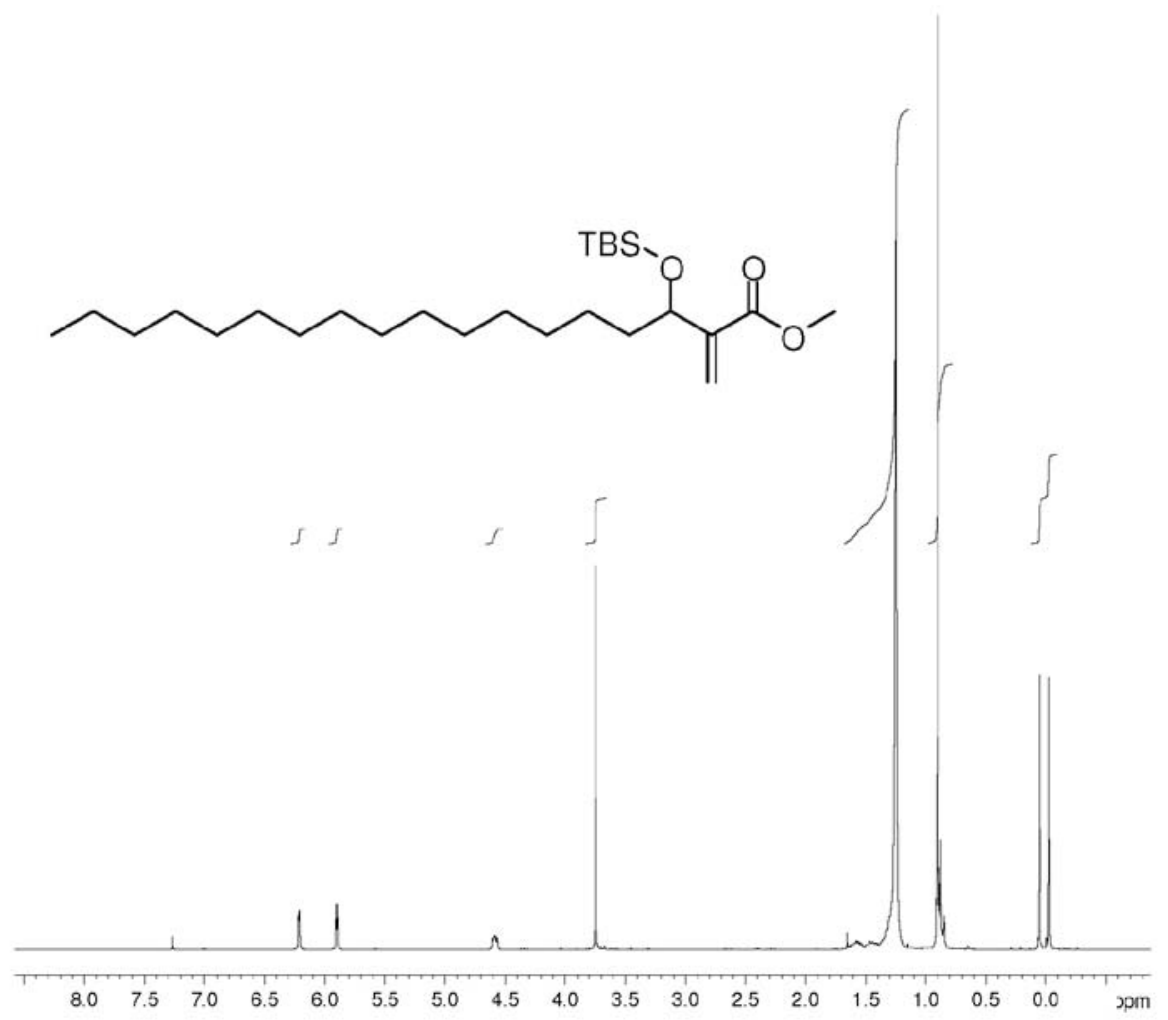

Figure S39. ${ }^{1} \mathrm{H}$ NMR $\left(\mathrm{CDCl}_{3}, 250 \mathrm{MHz}\right)$ of silylated $\mathrm{MBH}$ adduct 22. 


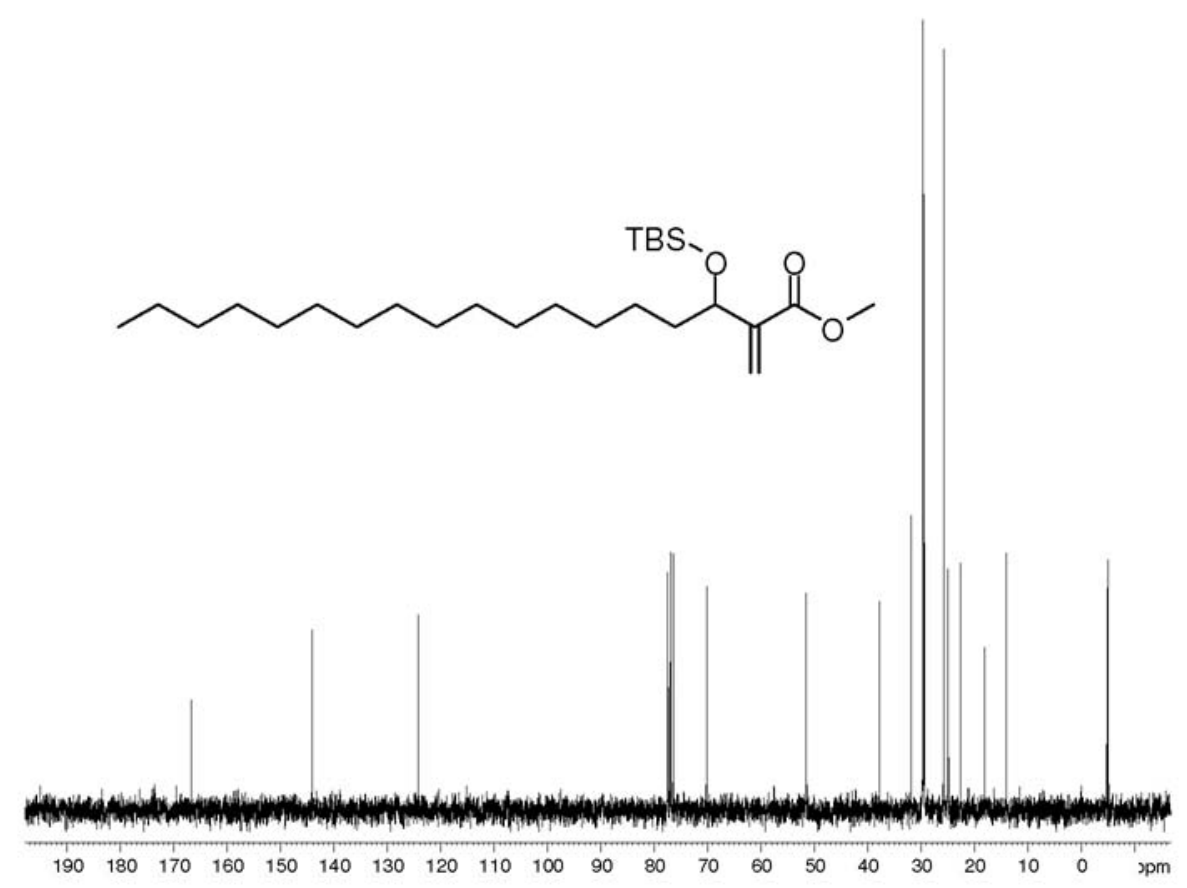

Figure S40. ${ }^{13} \mathrm{C}$ NMR $\left(\mathrm{CDCl}_{3}, 62.5 \mathrm{MHz}\right)$ of silylated $\mathrm{MBH}$ adduct 22.

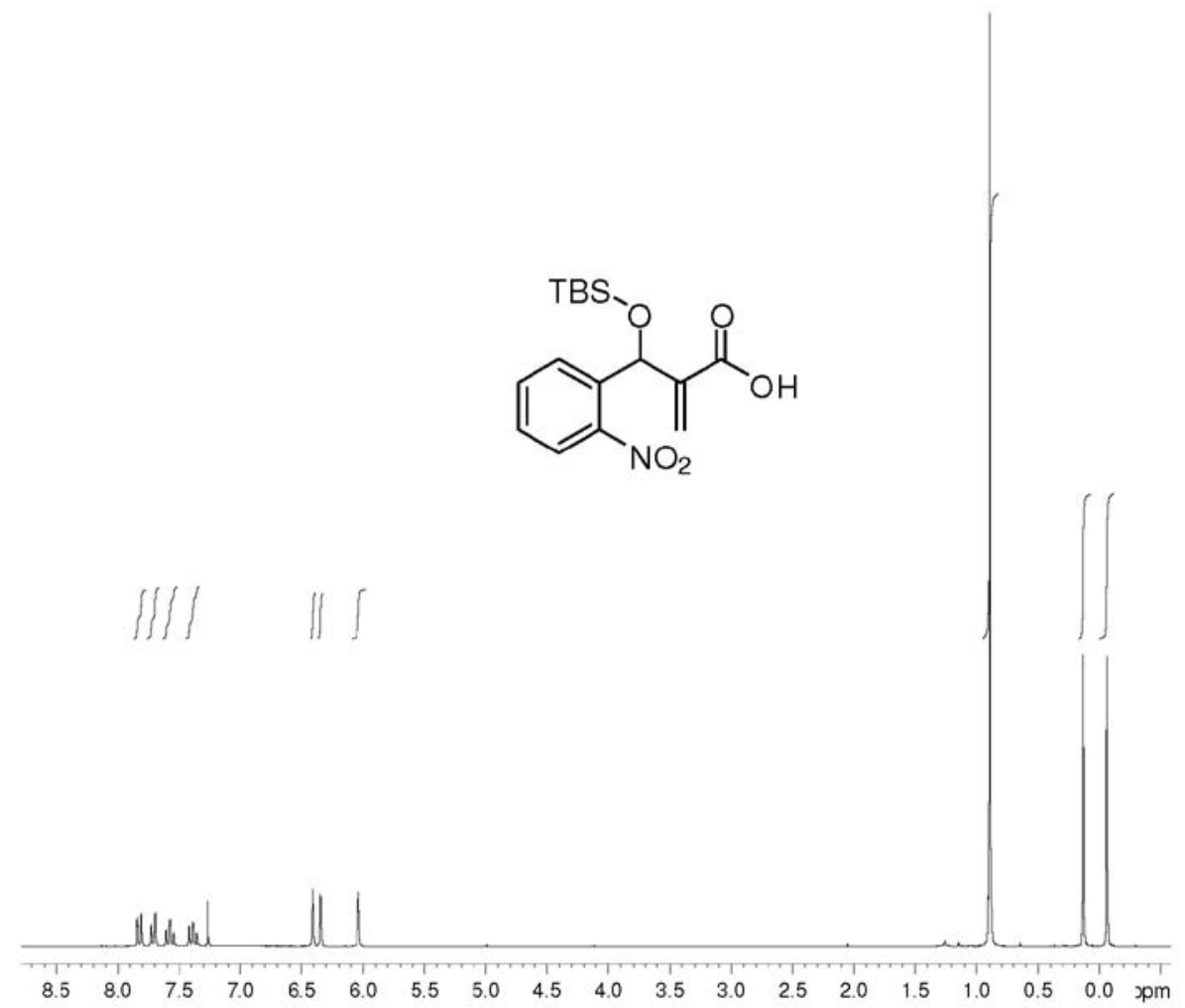

Figure S41. ${ }^{1} \mathrm{H} \mathrm{NMR}\left(\mathrm{CDCl}_{3}, 250 \mathrm{MHz}\right)$ of silylated acid 23. 


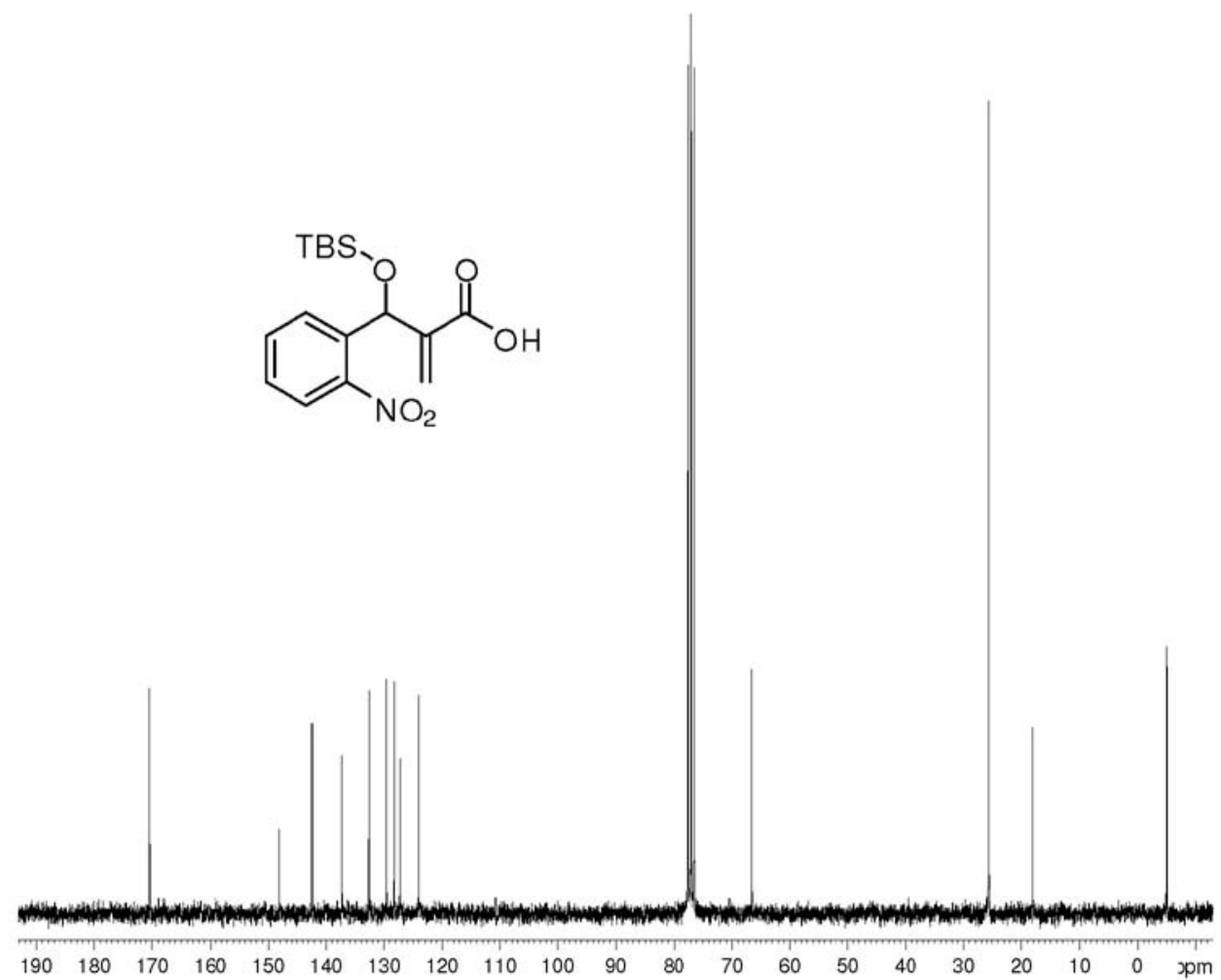

Figure S42. ${ }^{13} \mathrm{C}$ NMR $\left(\mathrm{CDCl}_{3}, 62.5 \mathrm{MHz}\right)$ of silylated acid 23.

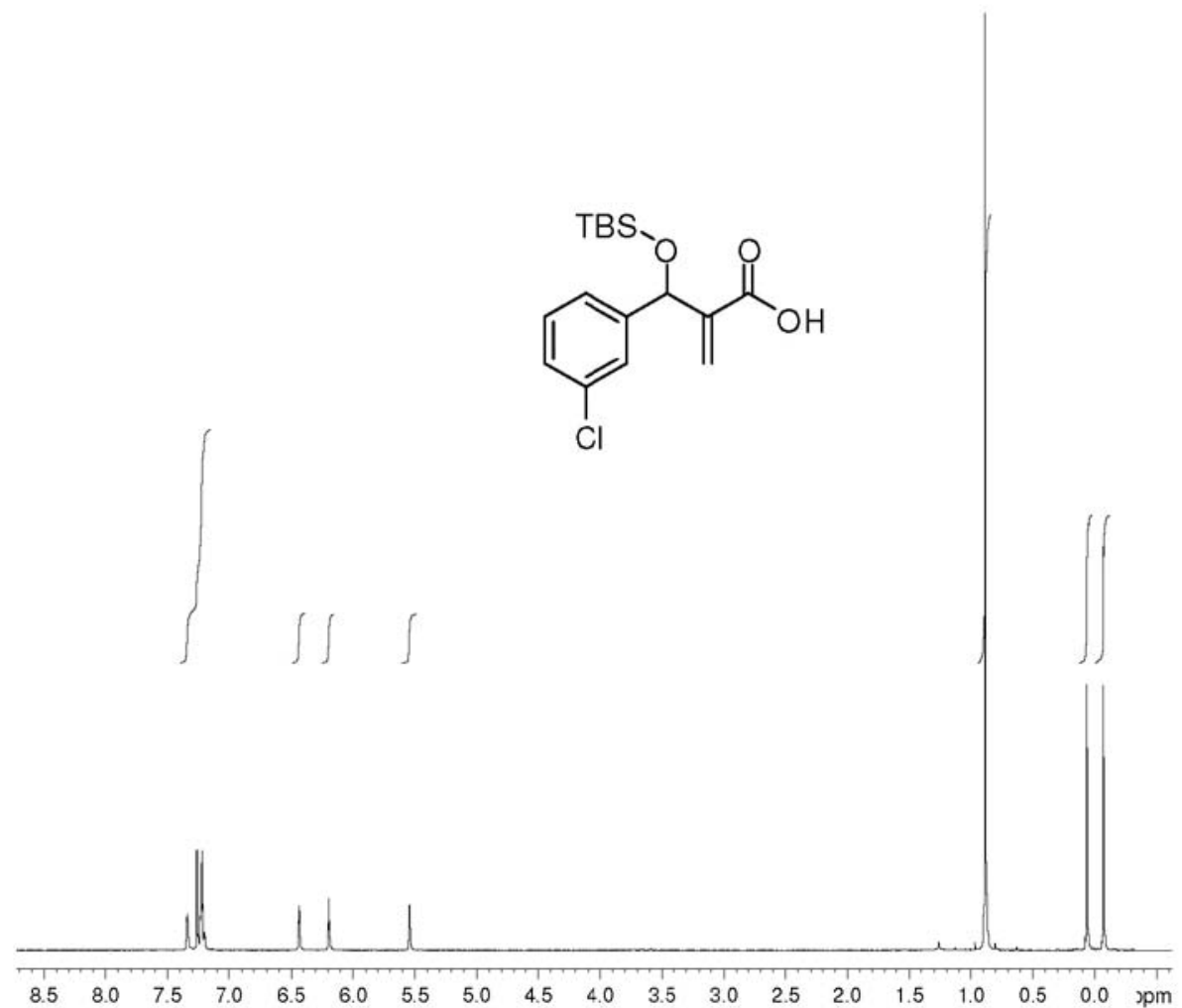

Figure S43. ${ }^{1} \mathrm{H}$ NMR $\left(\mathrm{CDCl}_{3}, 250 \mathrm{MHz}\right)$ of silylated acid 24. 
Vol. 22, No. 8, 2011

Amarante et al.

$\mathrm{S} 23$
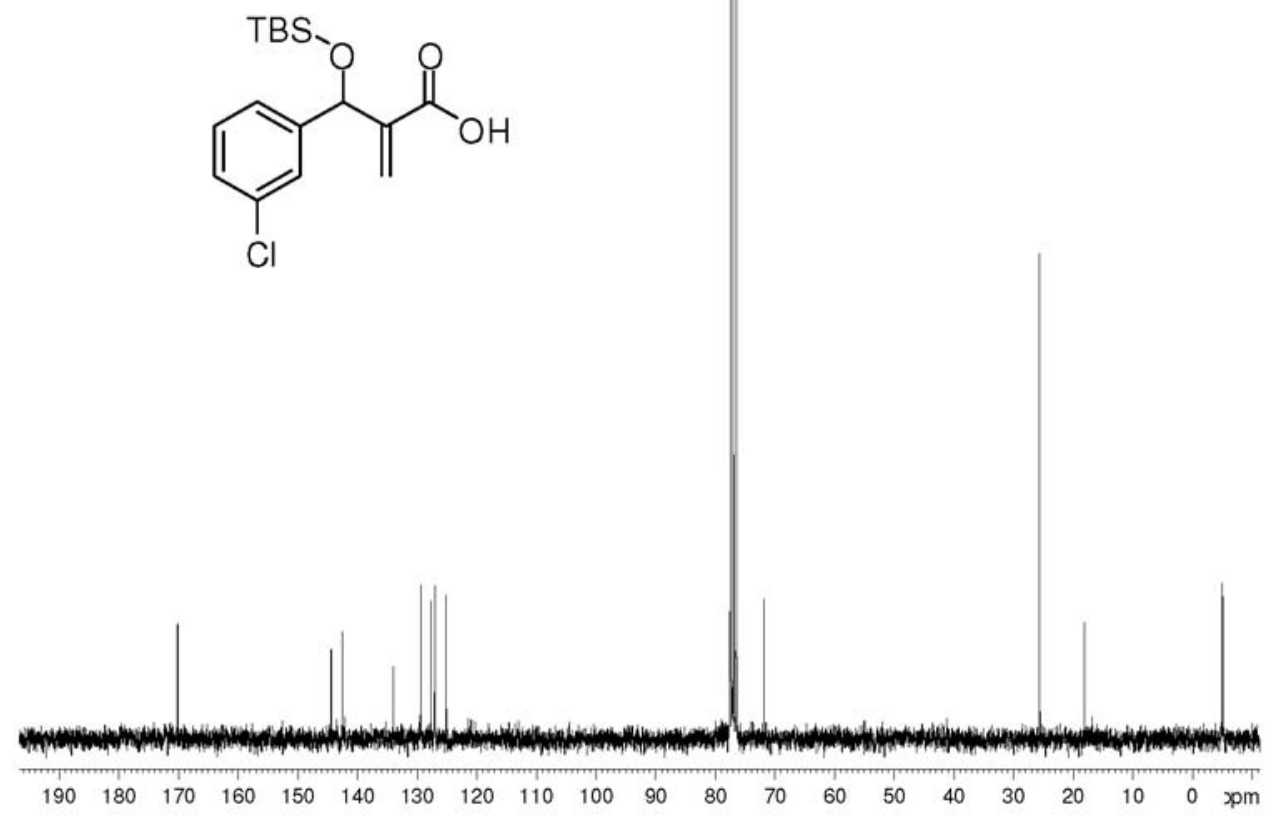

Figure S44. ${ }^{13} \mathrm{C} \mathrm{NMR}\left(\mathrm{CDCl}_{3}, 62.5 \mathrm{MHz}\right)$ of silylated acid 24 .

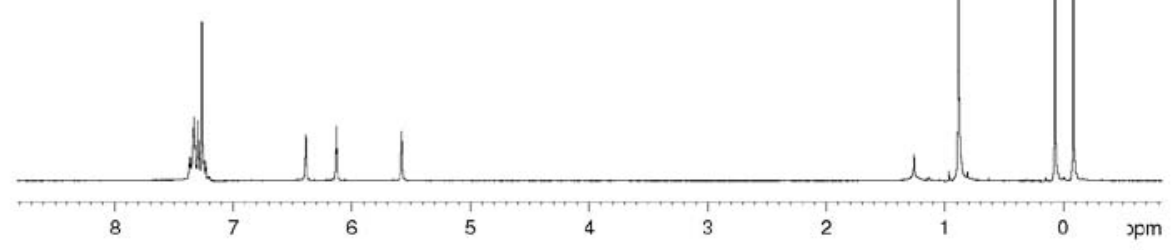

Figure S45. ${ }^{1} \mathrm{H}$ NMR $\left(\mathrm{CDCl}_{3}, 250 \mathrm{MHz}\right)$ of silylated acid 25 . 


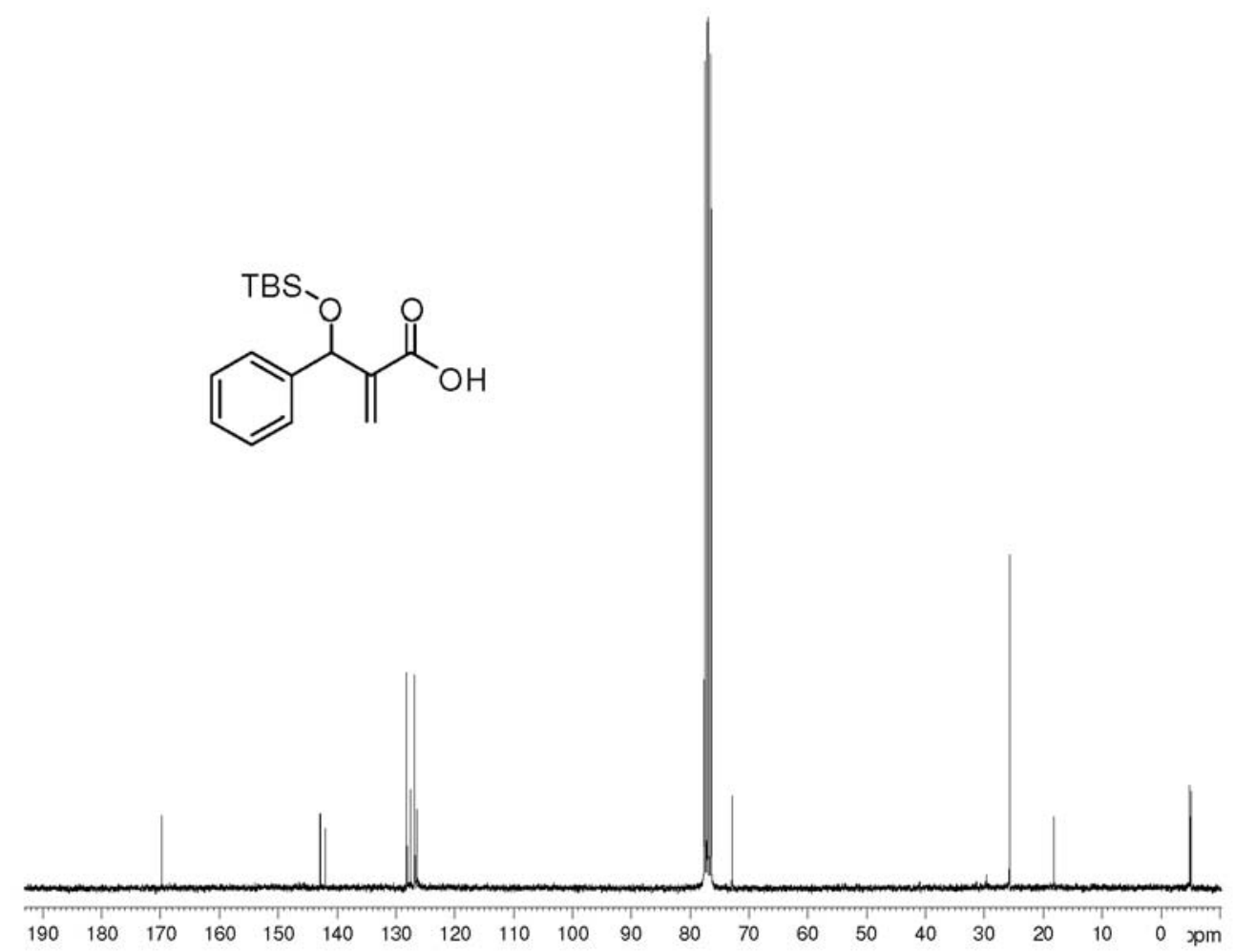

Figure S46. ${ }^{13} \mathrm{C} \mathrm{NMR}\left(\mathrm{CDCl}_{3}, 62.5 \mathrm{MHz}\right)$ of silylated acid 25 .

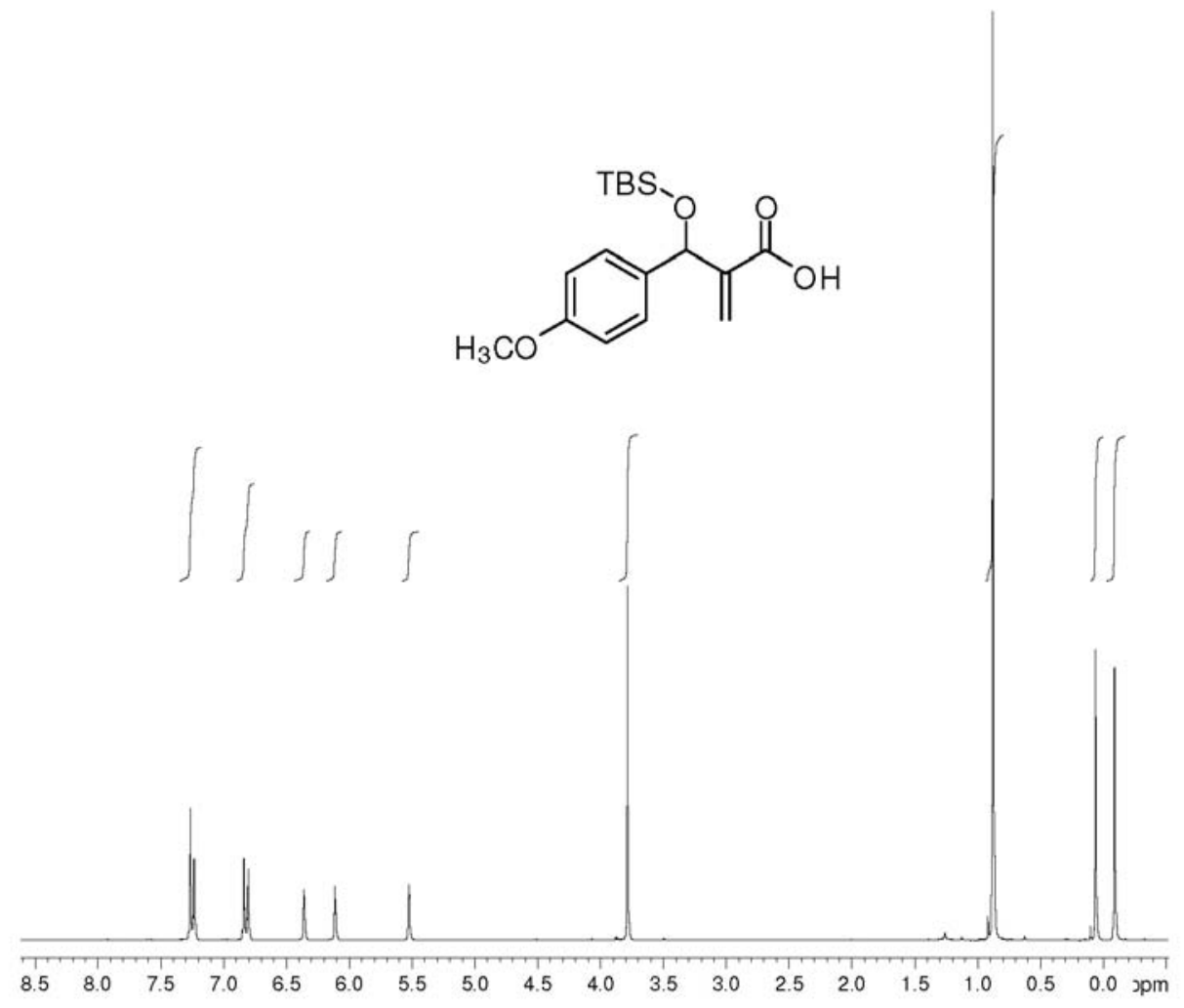

Figure S47. ${ }^{1} \mathrm{H}$ NMR $\left(\mathrm{CDCl}_{3}, 250 \mathrm{MHz}\right)$ of silylated acid 26. 


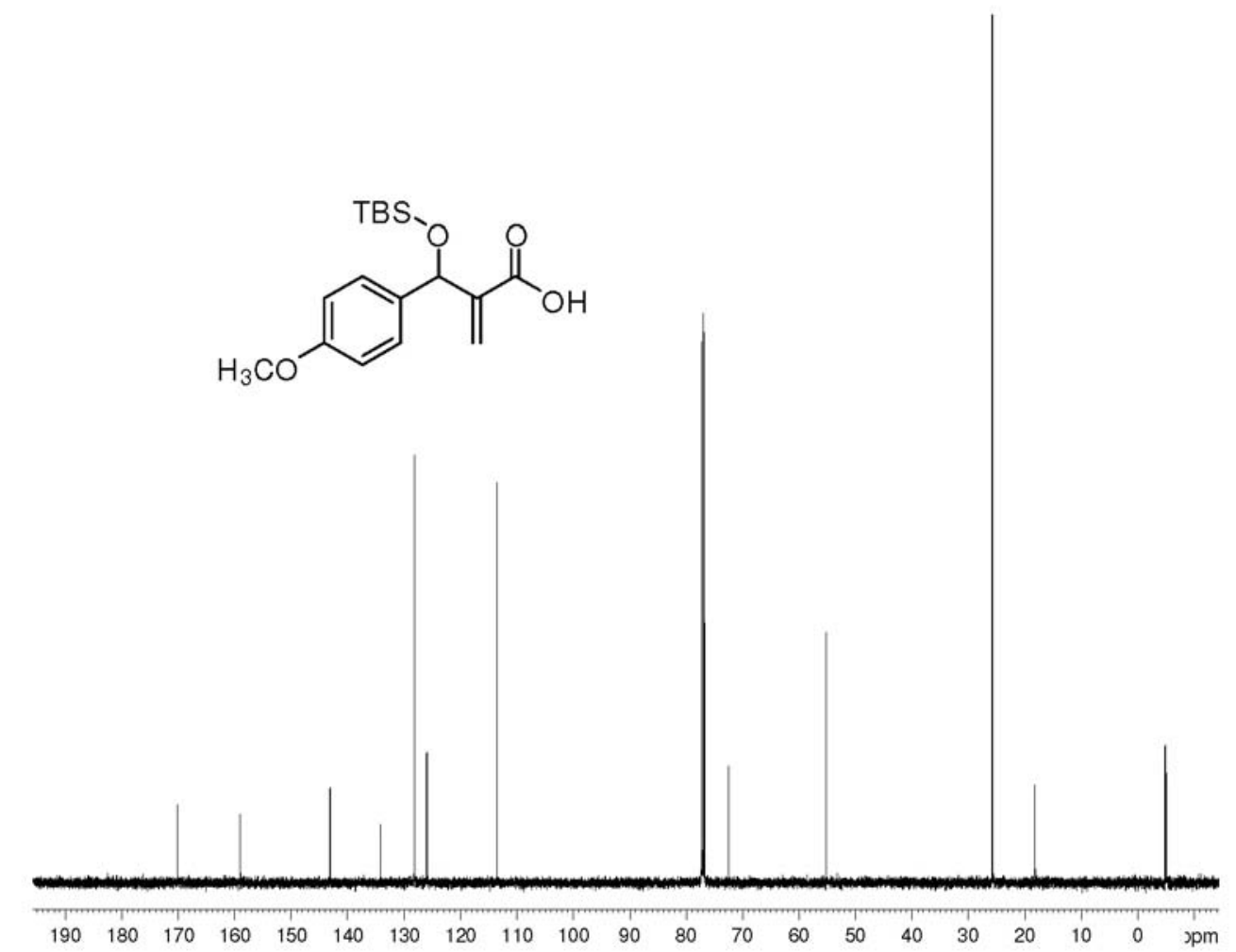

Figure S48. ${ }^{13} \mathrm{C}$ NMR $\left(\mathrm{CDCl}_{3}, 125 \mathrm{MHz}\right)$ of silylated acid 26.

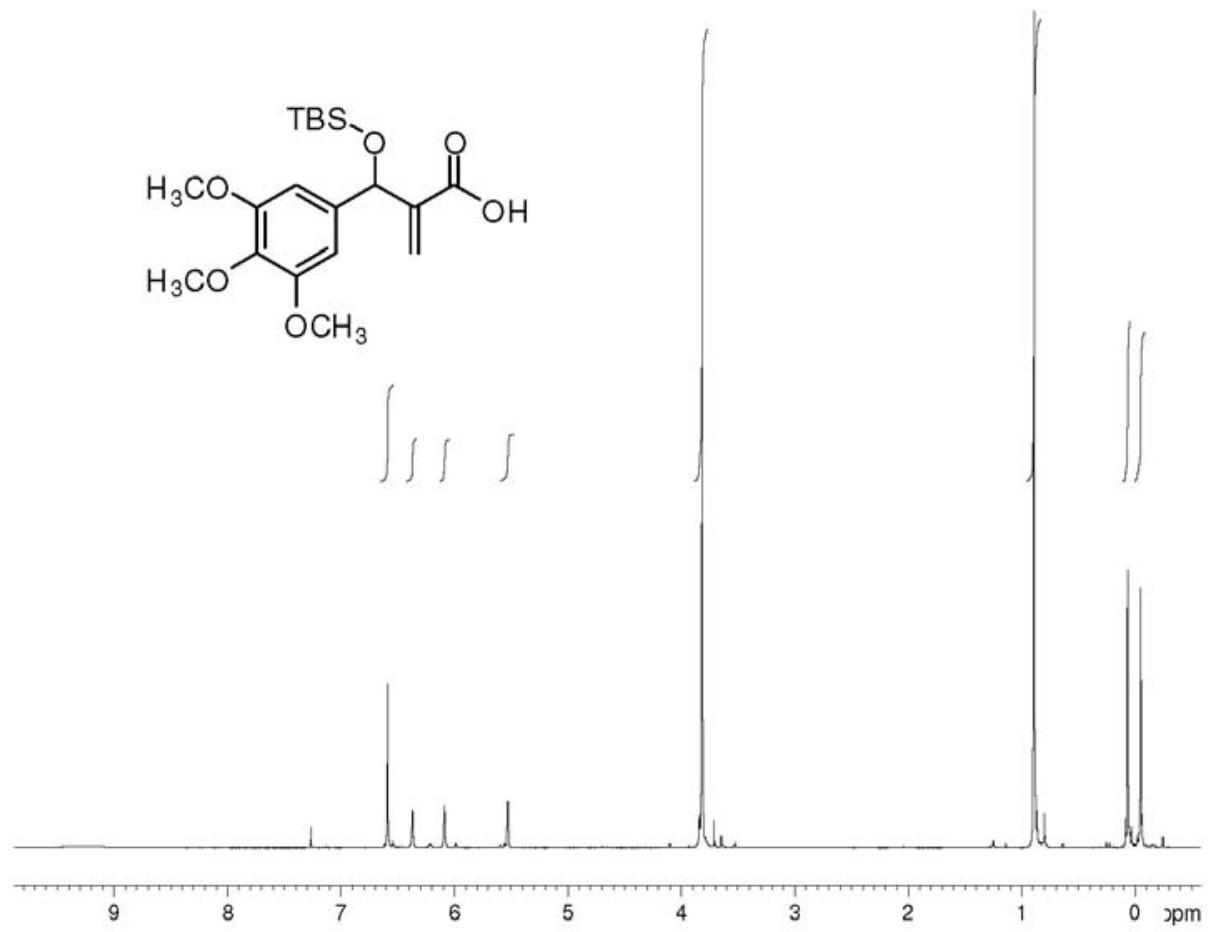

Figure S49. ${ }^{1} \mathrm{H}$ NMR $\left(\mathrm{CDCl}_{3}, 250 \mathrm{MHz}\right)$ of silylated acid 27. 


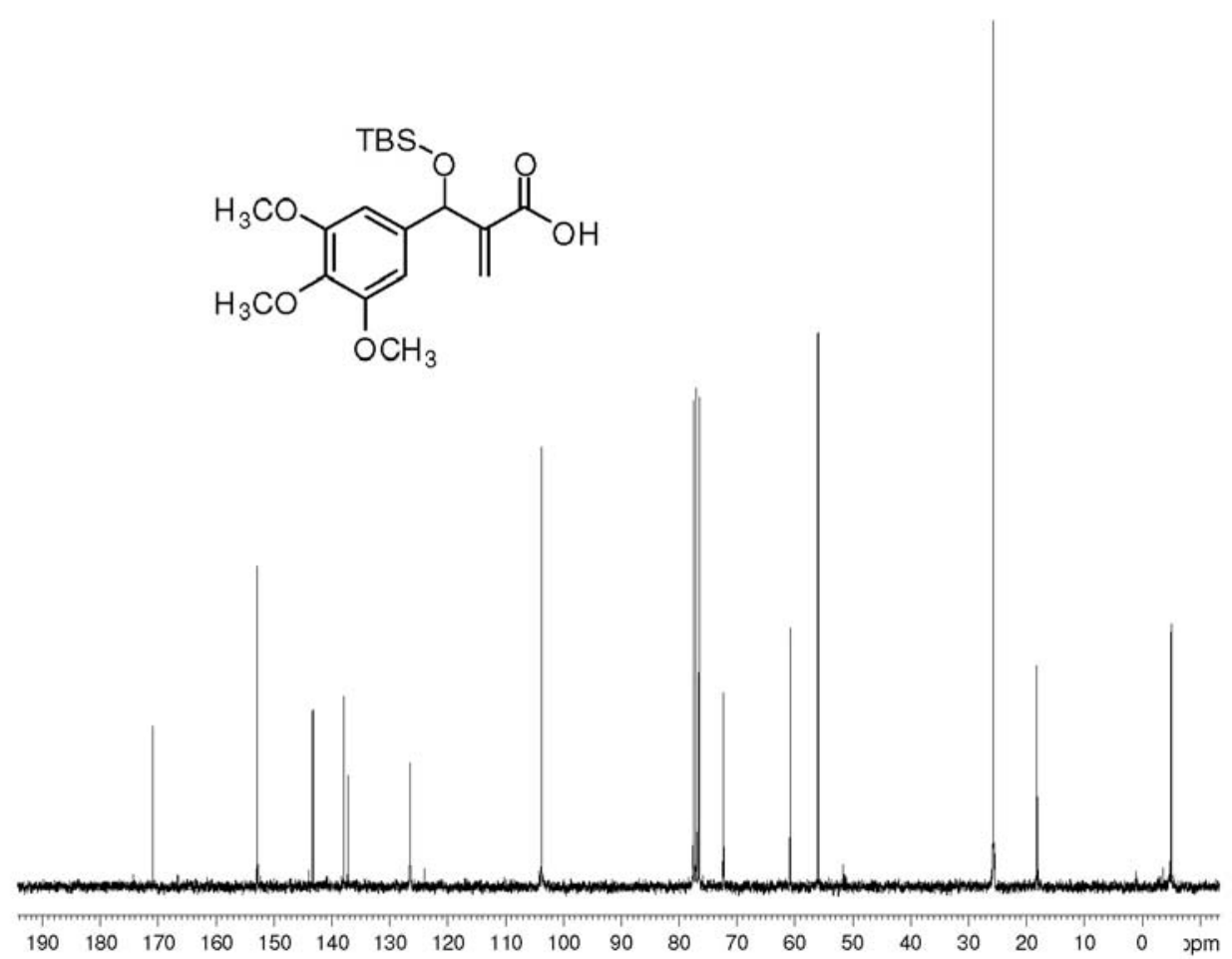

Figure S50. ${ }^{13} \mathrm{C}$ NMR $\left(\mathrm{CDCl}_{3}, 62.5 \mathrm{MHz}\right)$ of silylated acid 27.

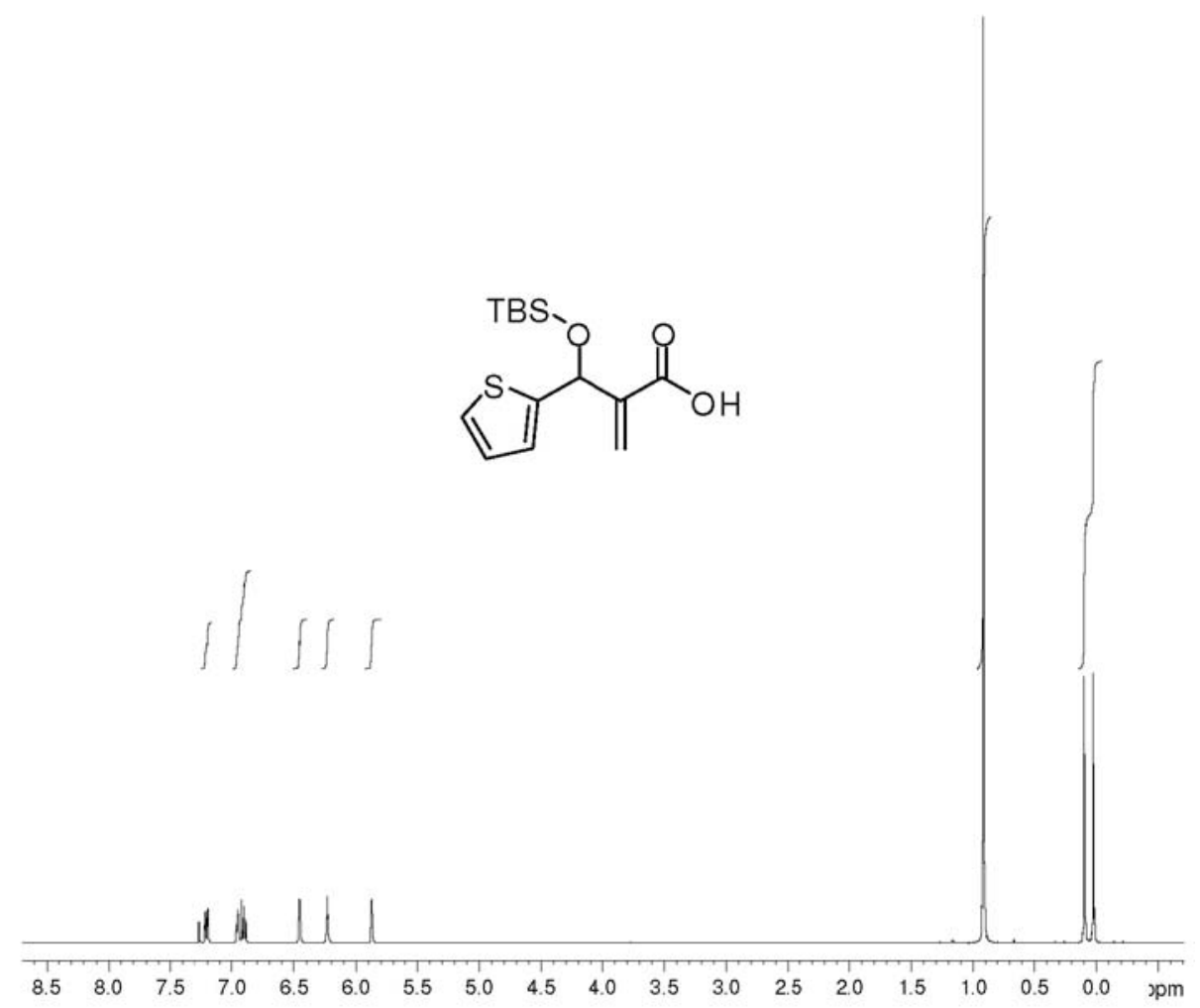

Figure S51. ${ }^{1} \mathrm{H}$ NMR $\left(\mathrm{CDCl}_{3}, 250 \mathrm{MHz}\right)$ of silylated acid 28. 


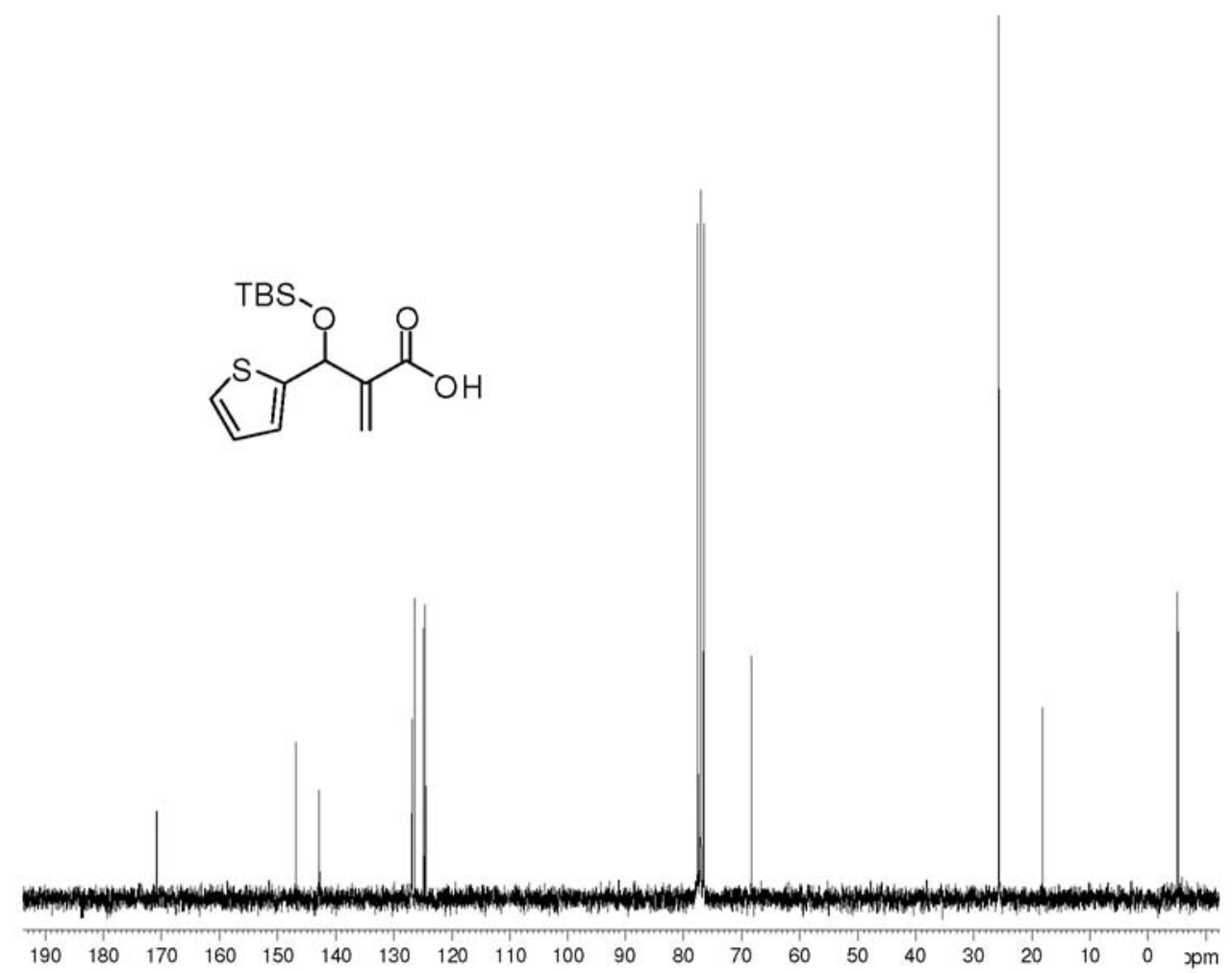

Figure S52. ${ }^{13} \mathrm{C}$ NMR $\left(\mathrm{CDCl}_{3}, 62.5 \mathrm{MHz}\right)$ of silyated acid 28.

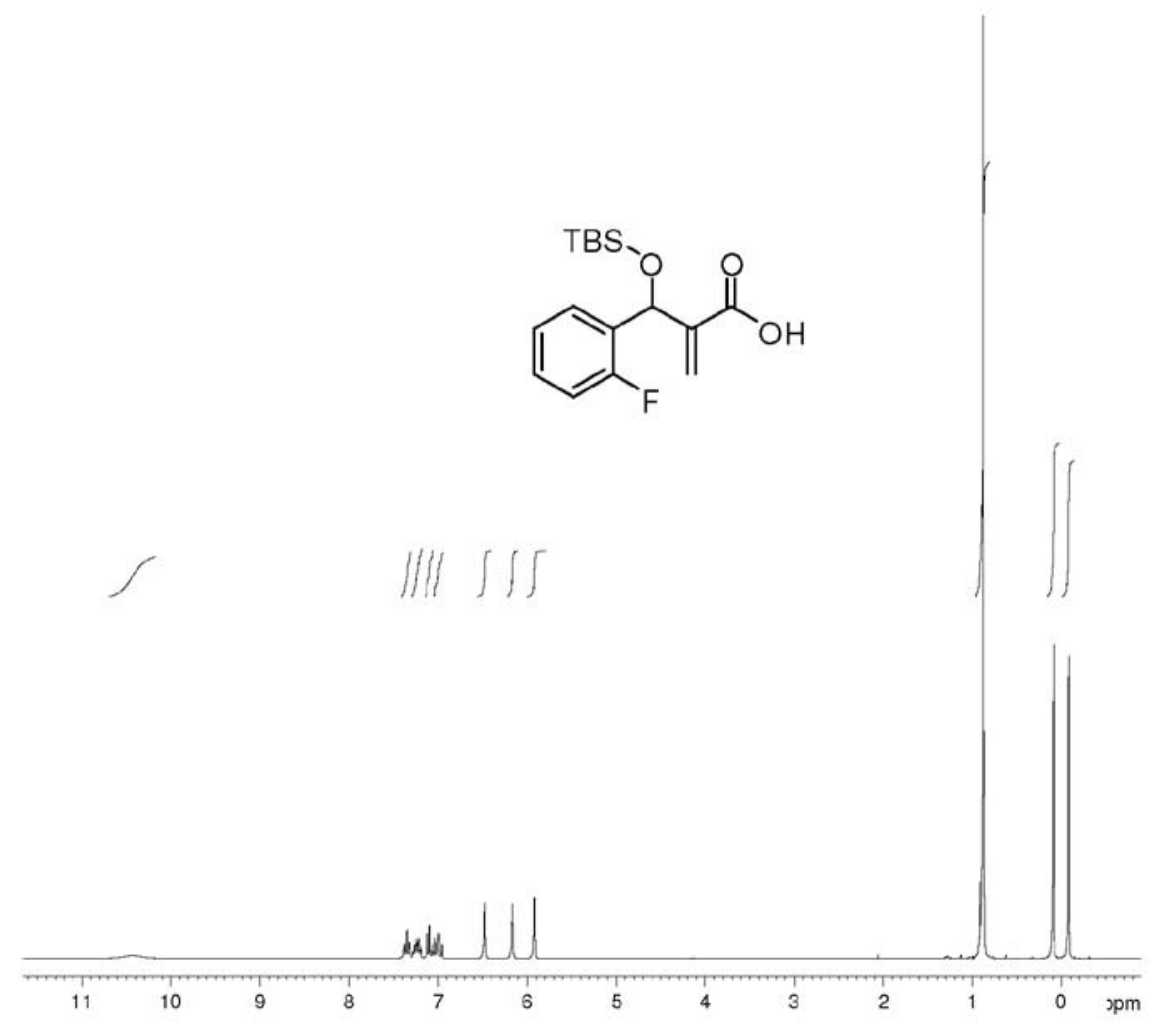

Figure S53. ${ }^{1} \mathrm{H}$ NMR $\left(\mathrm{CDCl}_{3}, 250 \mathrm{MHz}\right)$ of silylated acid 29. 


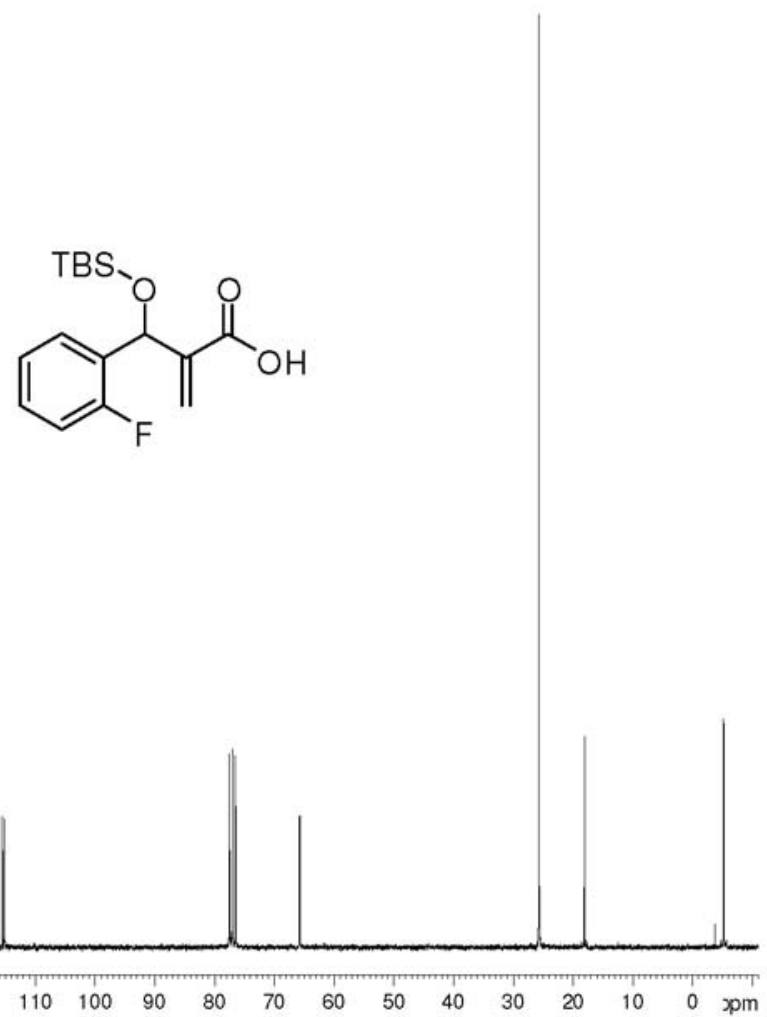

Figure S54. ${ }^{13} \mathrm{C}$ NMR $\left(\mathrm{CDCl}_{3}, 62.5 \mathrm{MHz}\right)$ of silylated acid 29.

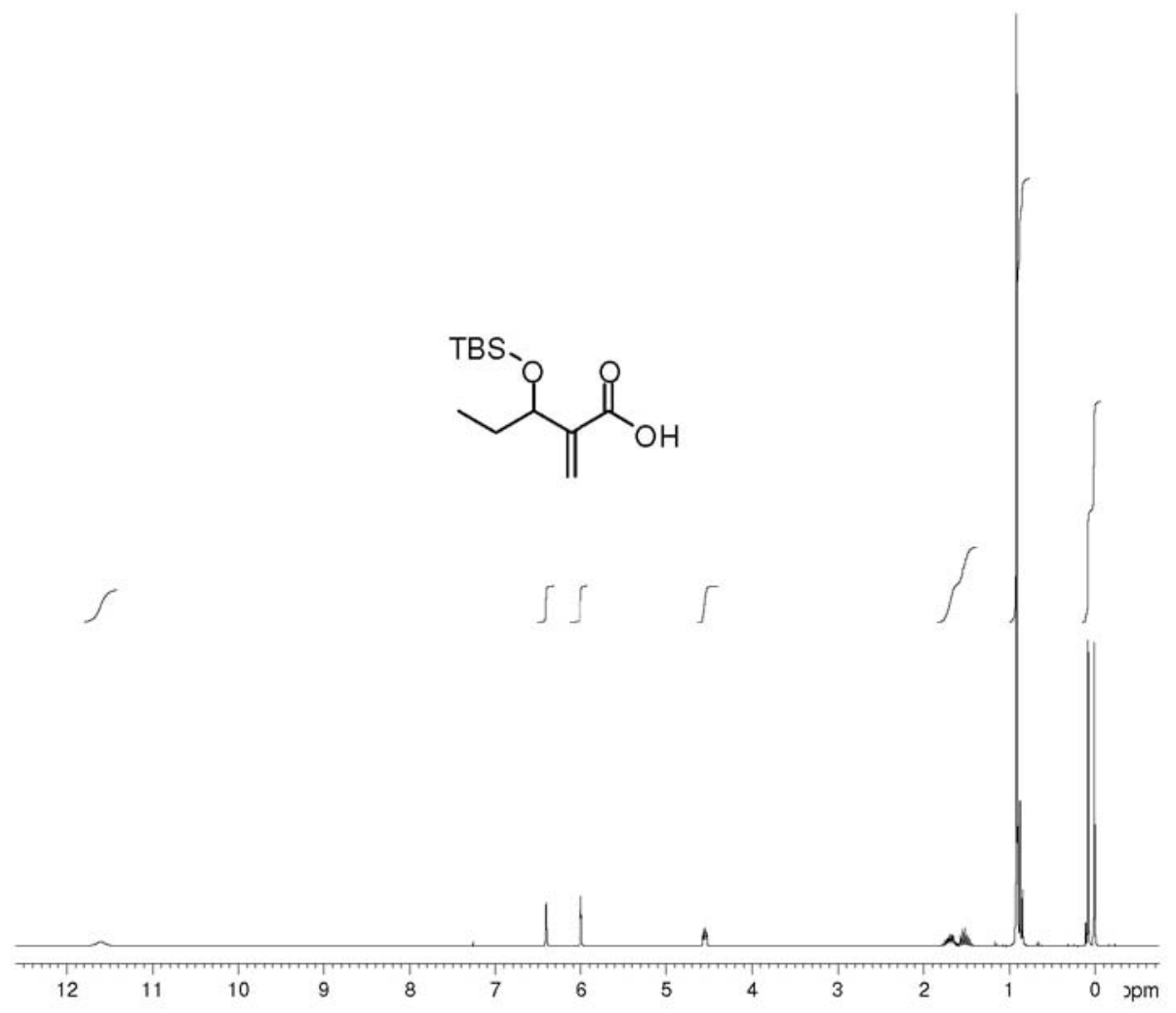

Figure S55. ${ }^{1} \mathrm{H}$ NMR $\left(\mathrm{CDCl}_{3}, 250 \mathrm{MHz}\right)$ of silylated acid $\mathbf{3 0}$. 


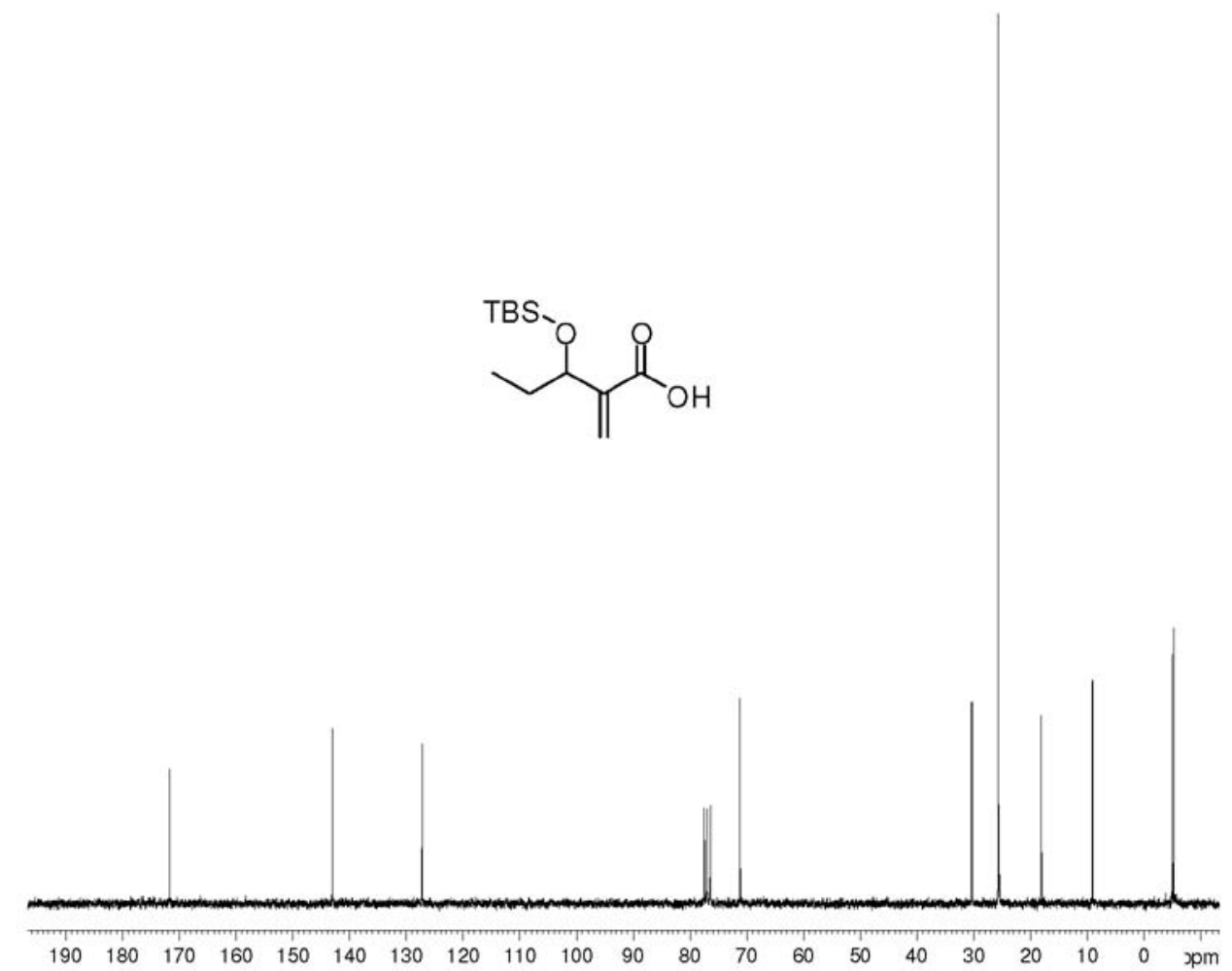

Figure S56. ${ }^{13} \mathrm{C}$ NMR $\left(\mathrm{CDCl}_{3}, 62.5 \mathrm{MHz}\right)$ of silylated acid $\mathbf{3 0}$.

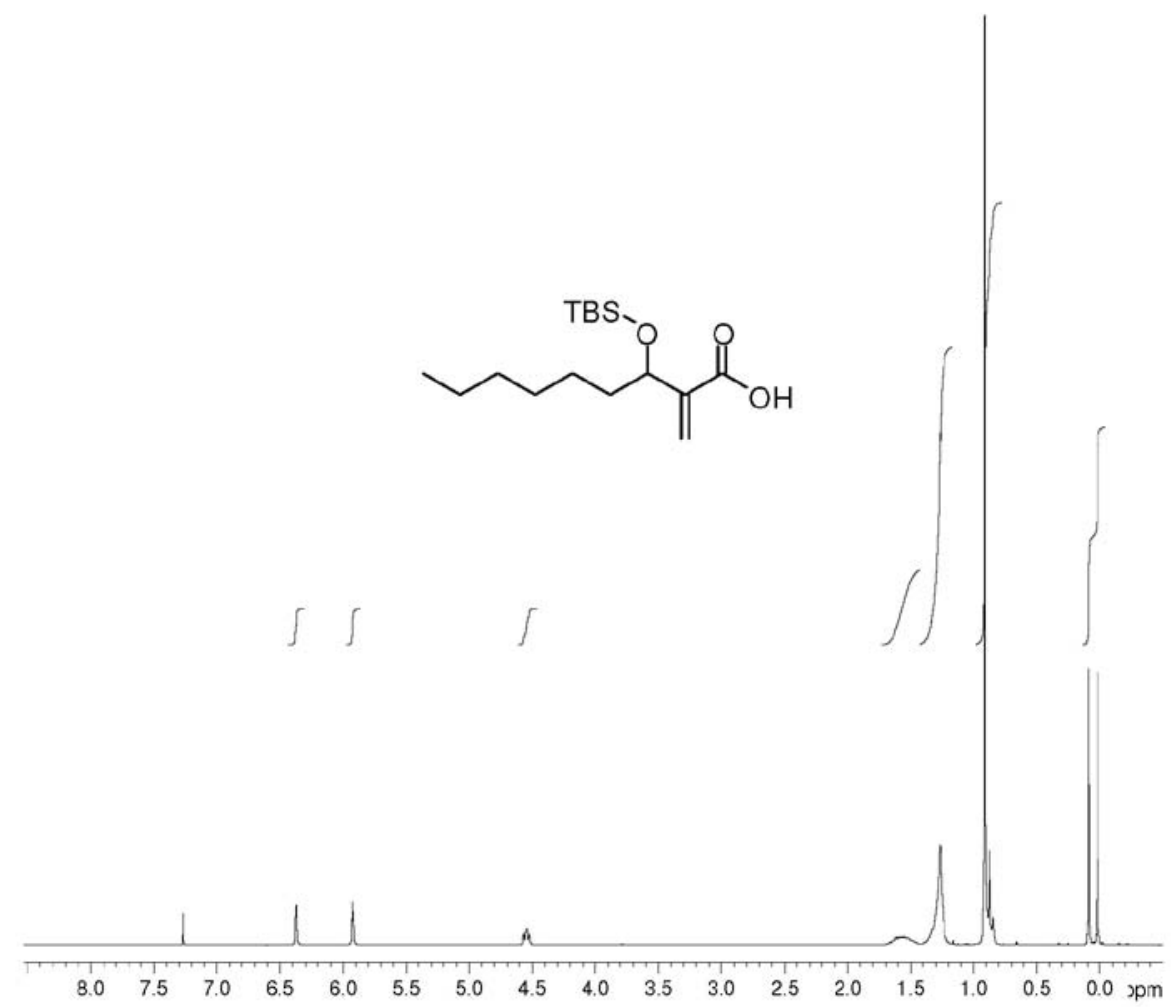

Figure S57. ${ }^{1} \mathrm{H}$ NMR $\left(\mathrm{CDCl}_{3}, 250 \mathrm{MHz}\right)$ of silylated acid $\mathbf{3 1}$. 


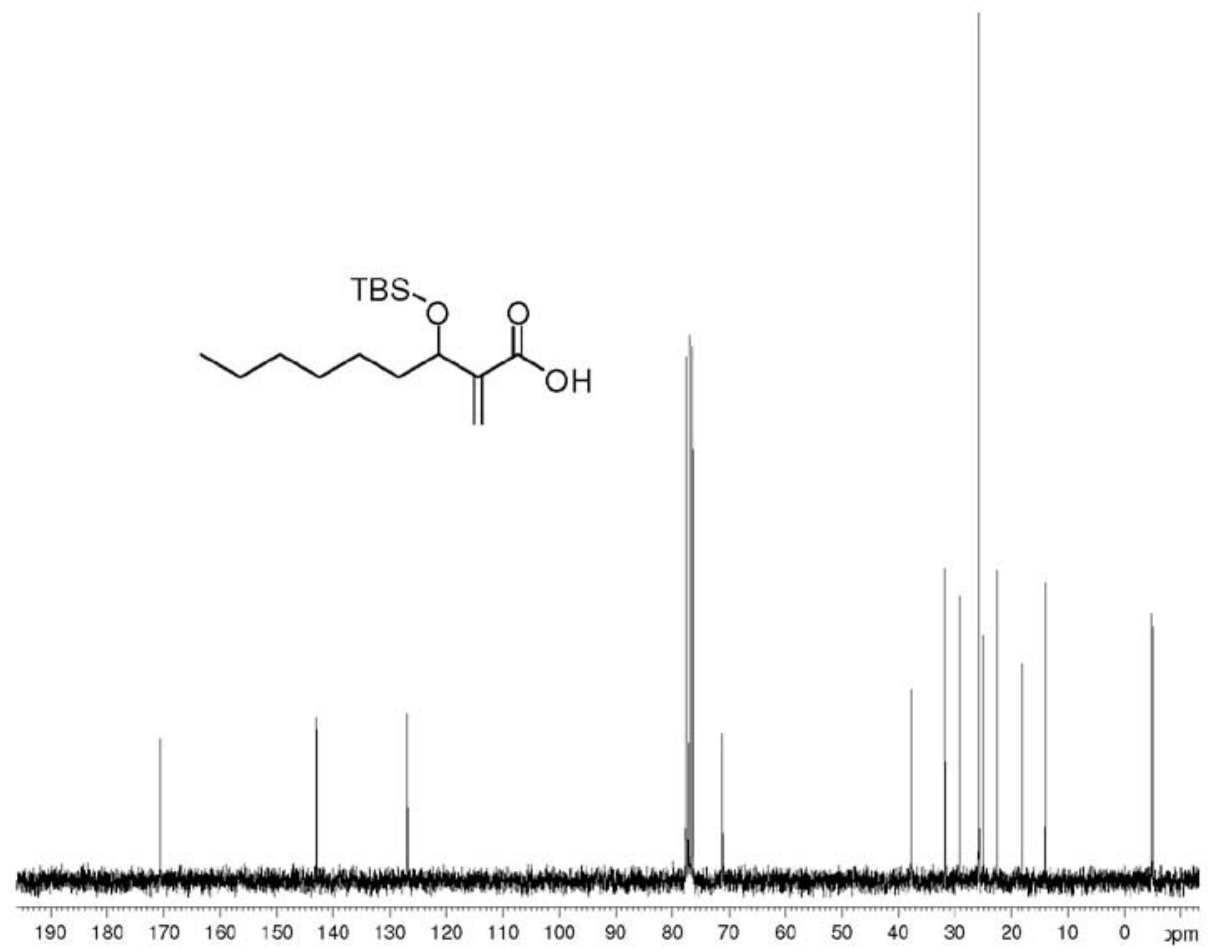

Figure S58. ${ }^{13} \mathrm{C}$ NMR $\left(\mathrm{CDCl}_{3}, 62.5 \mathrm{MHz}\right)$ of silylated acid $\mathbf{3 1}$.

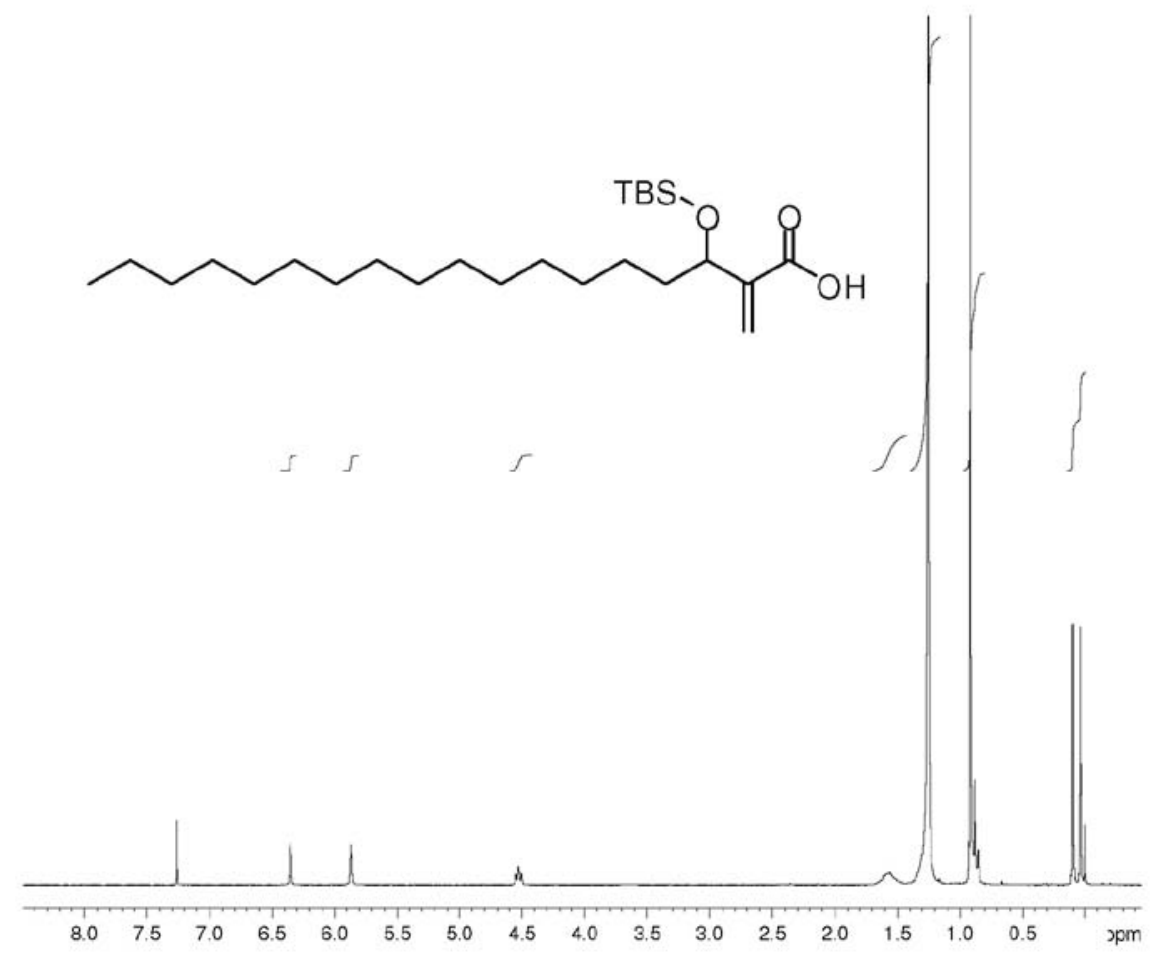

Figure S59. ${ }^{1} \mathrm{H}$ NMR $\left(\mathrm{CDCl}_{3}, 250 \mathrm{MHz}\right)$ of silylated acid $\mathbf{3 2}$. 


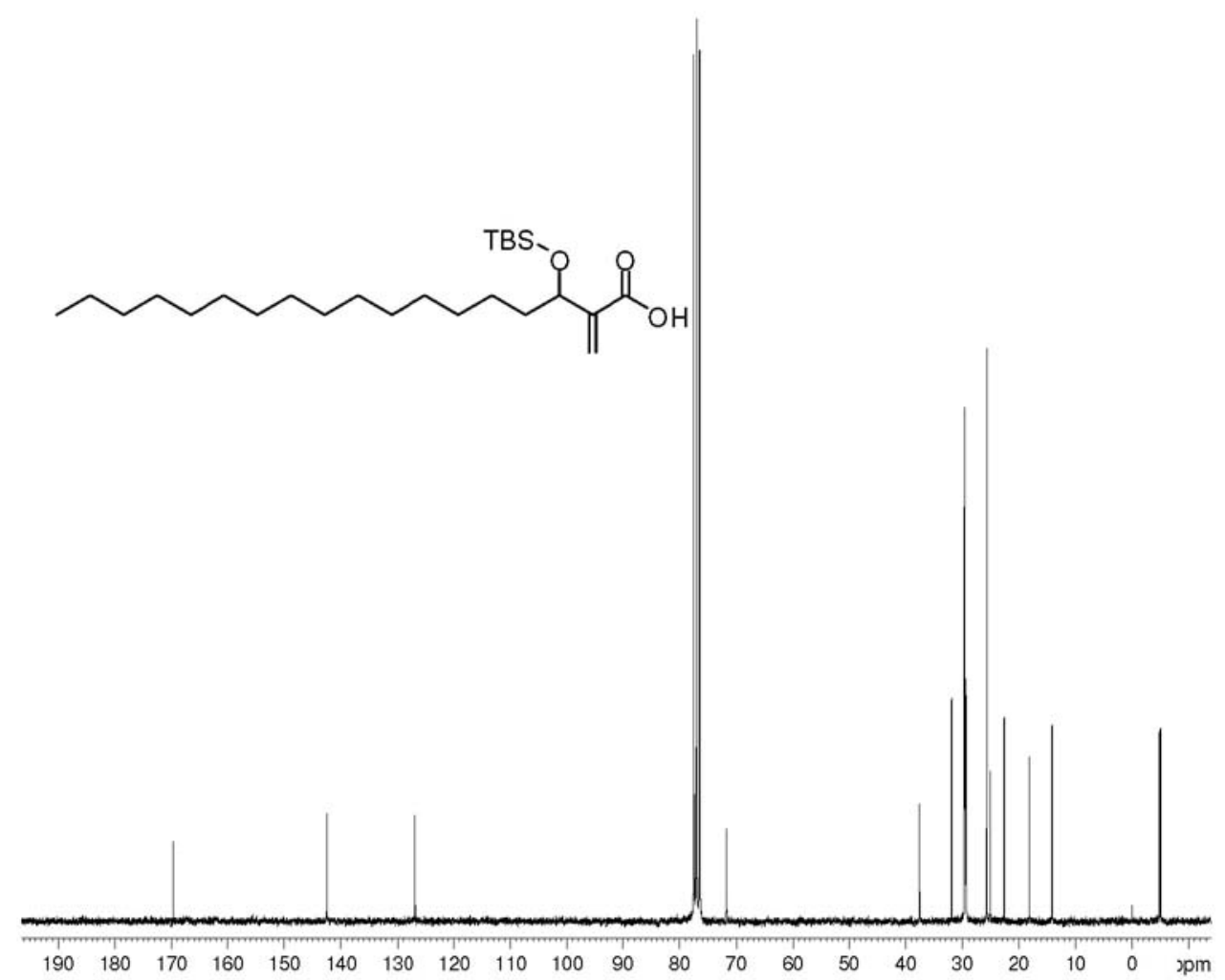

Figure S60. ${ }^{13} \mathrm{C} \mathrm{NMR}\left(\mathrm{CDCl}_{3}, 62.5 \mathrm{MHz}\right)$ of silylated acid 32 .

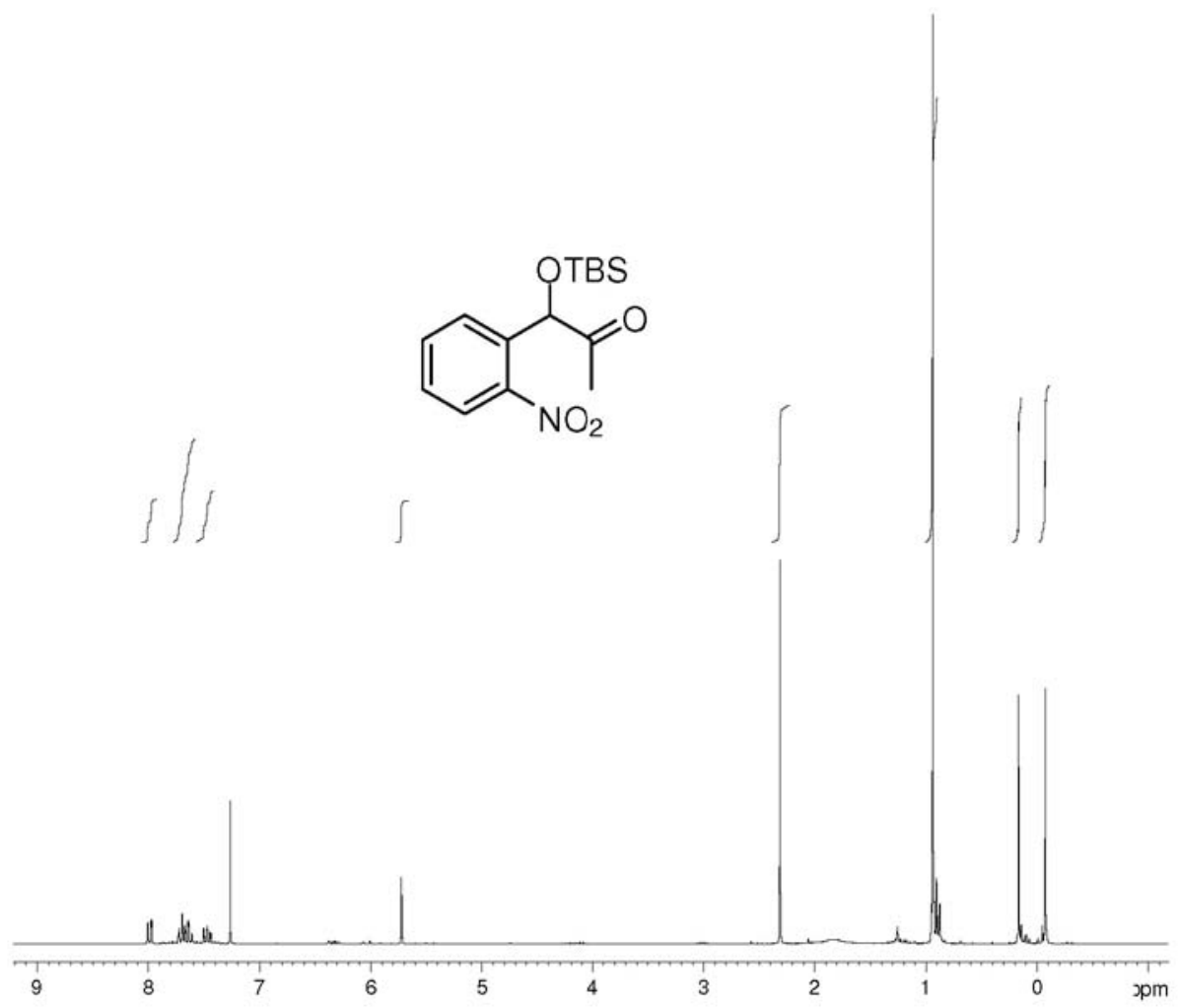

Figure S61. ${ }^{1} \mathrm{H}$ NMR $\left(\mathrm{CDCl}_{3}, 250 \mathrm{MHz}\right)$ of acyloin 33. 


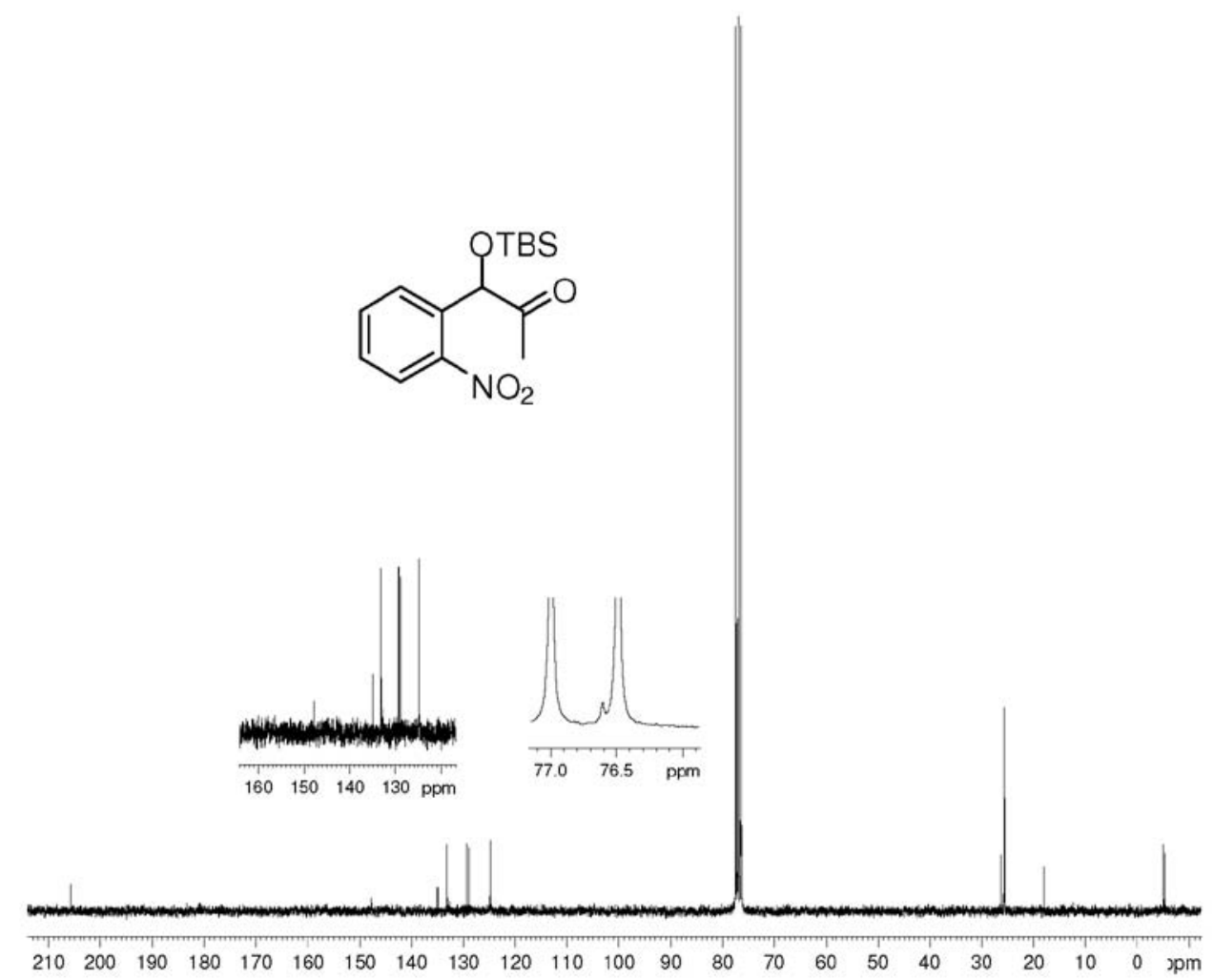

Figure S62. ${ }^{13} \mathrm{C}$ NMR $\left(\mathrm{CDCl}_{3}, 62.5 \mathrm{MHz}\right)$ of acyloin 33 .

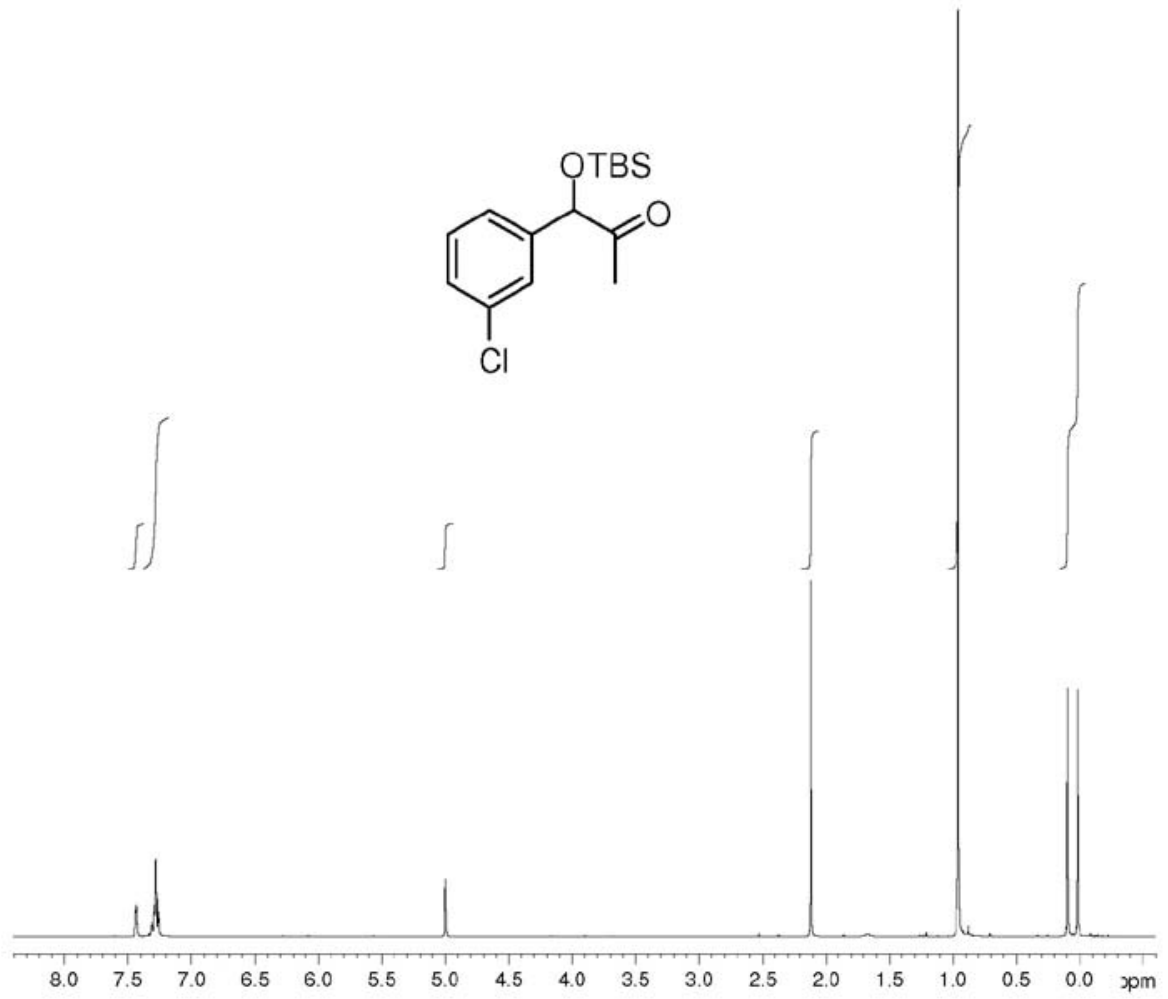

Figure S63. ${ }^{1} \mathrm{H}$ NMR $\left(\mathrm{CDCl}_{3}, 250 \mathrm{MHz}\right)$ of acyloin 34. 


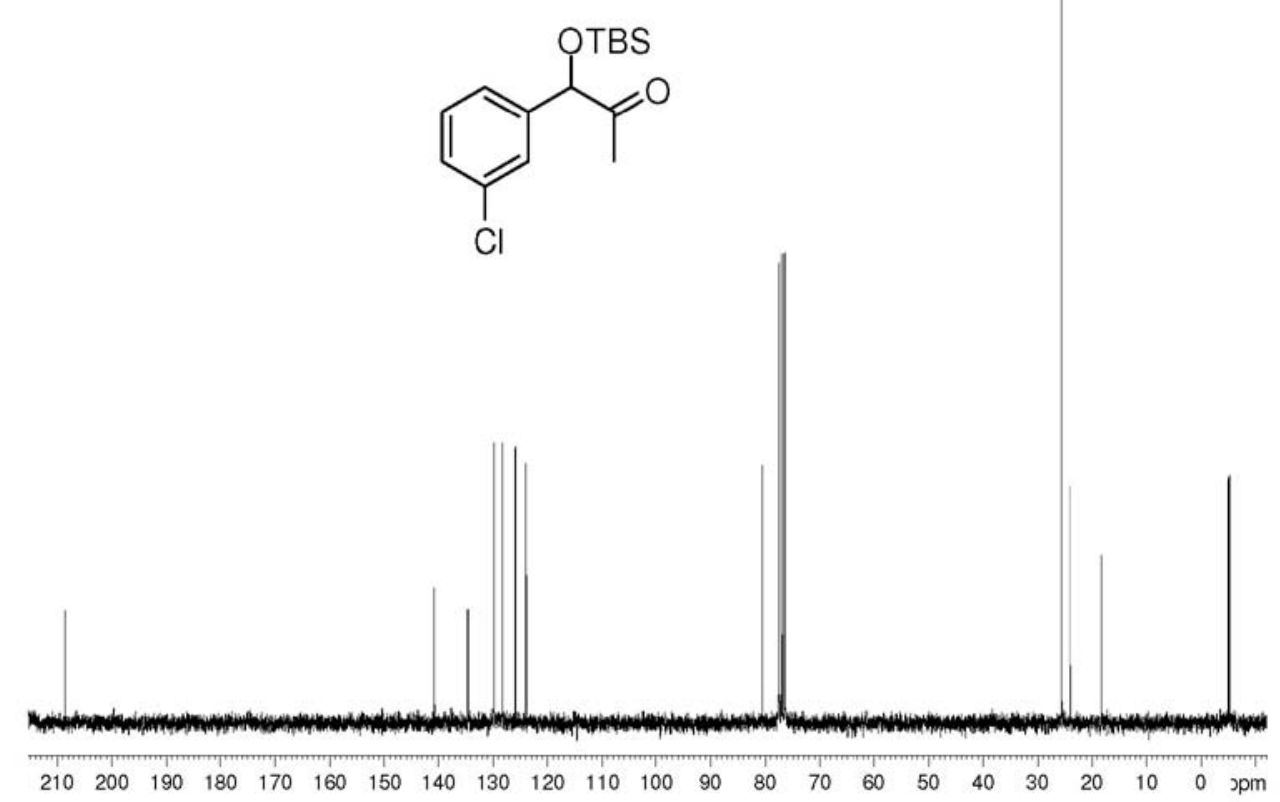

Figure S64. ${ }^{13} \mathrm{C} \mathrm{NMR}\left(\mathrm{CDCl}_{3}, 62.5 \mathrm{MHz}\right)$ of acyloin 34.

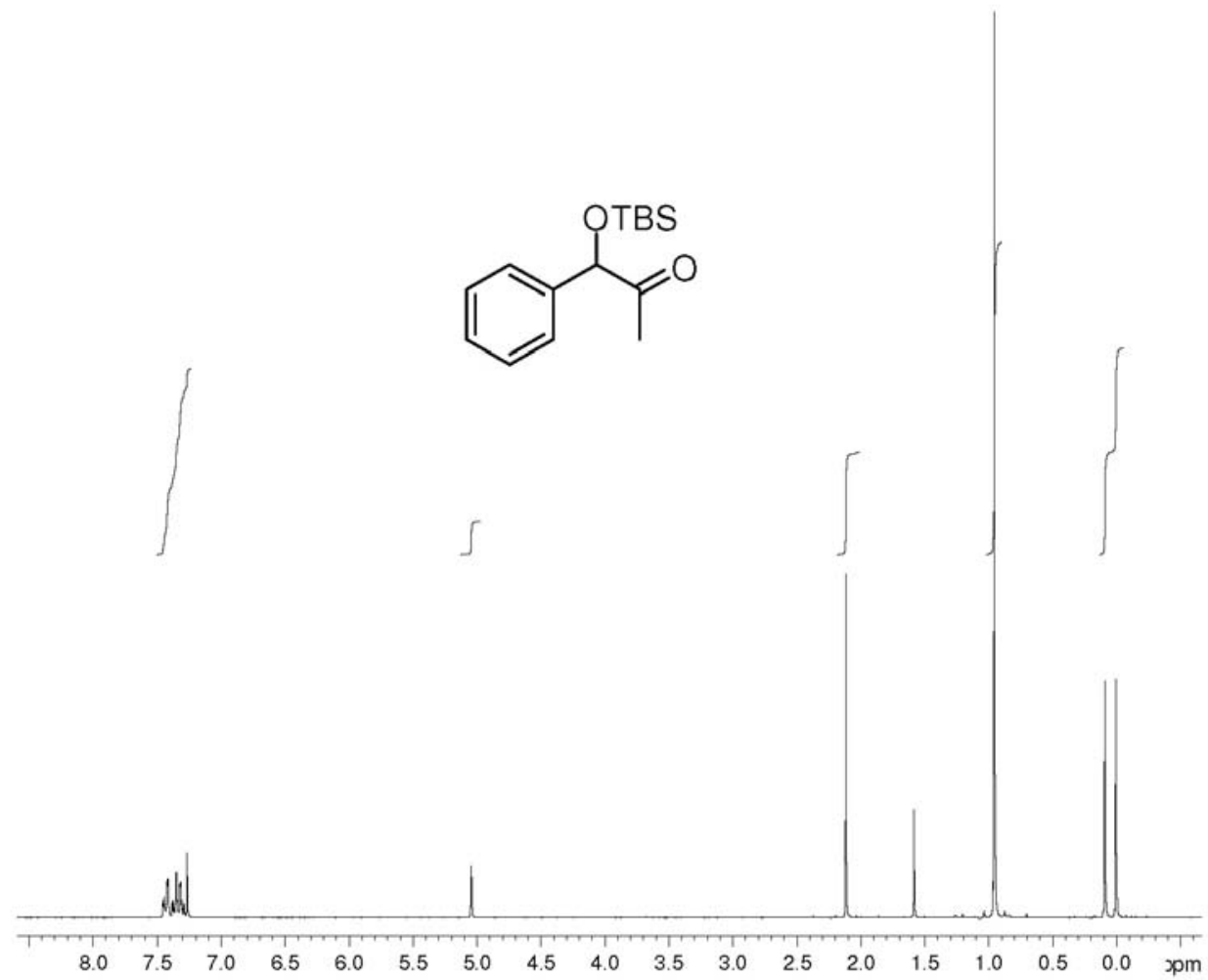

Figure S65. ${ }^{1} \mathrm{H}$ NMR $\left(\mathrm{CDCl}_{3}, 250 \mathrm{MHz}\right)$ of acyloin 35. 


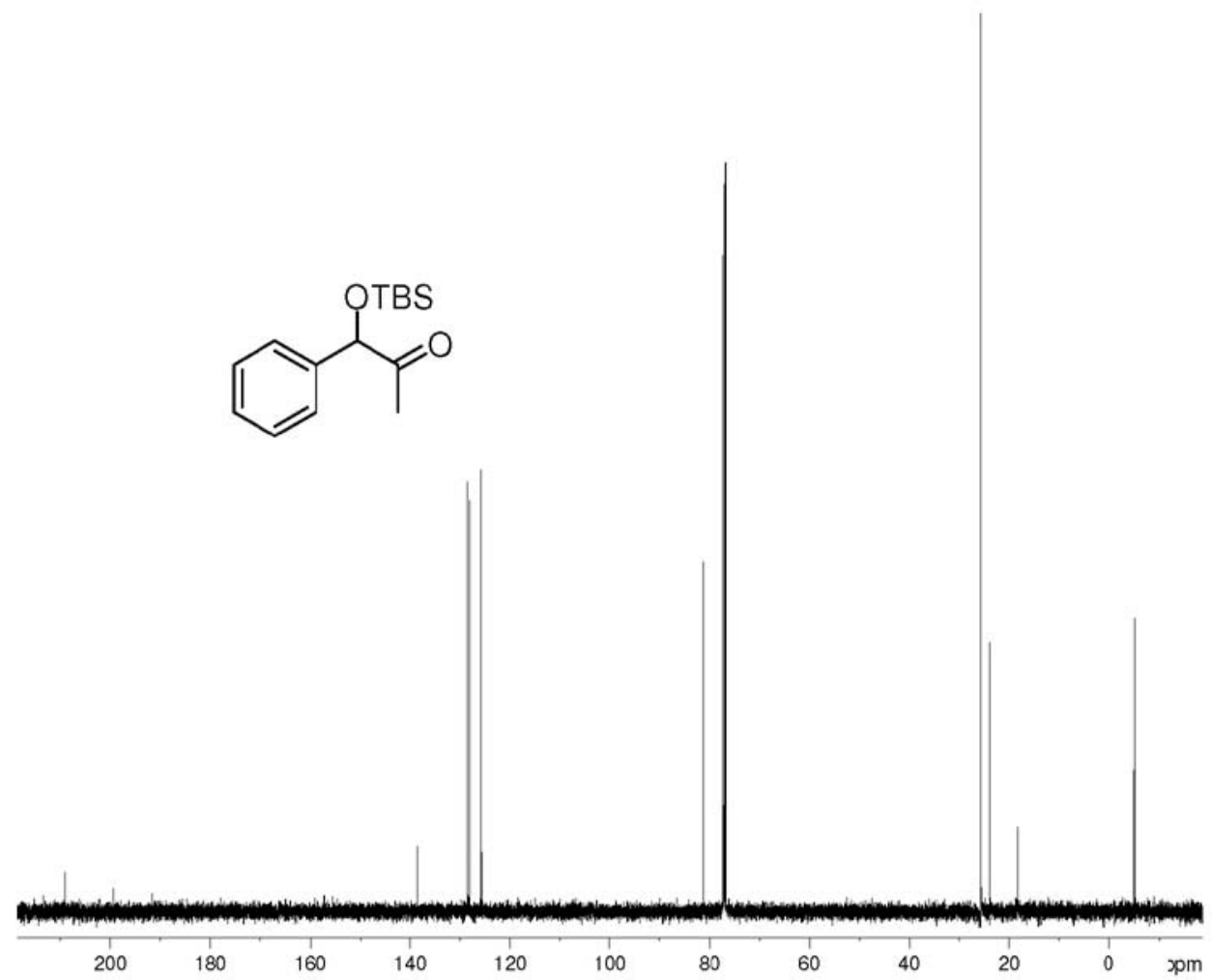

Figure S66. ${ }^{13} \mathrm{C}$ NMR $\left(\mathrm{CDCl}_{3}, 125 \mathrm{MHz}\right)$ of acyloin 35.

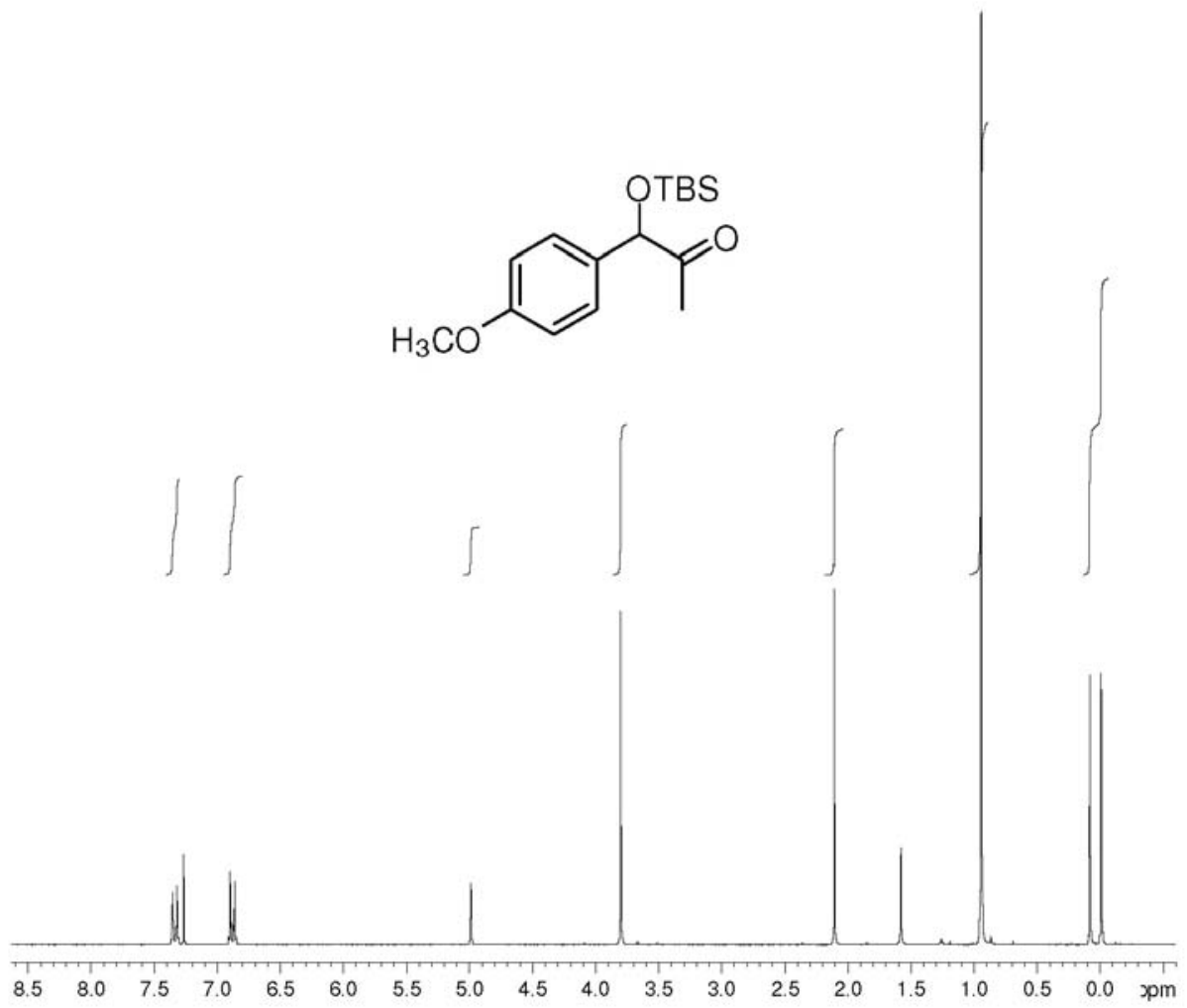

Figure S67. ${ }^{1} \mathrm{H}$ NMR $\left(\mathrm{CDCl}_{3}, 250 \mathrm{MHz}\right)$ of acyloin 36. 


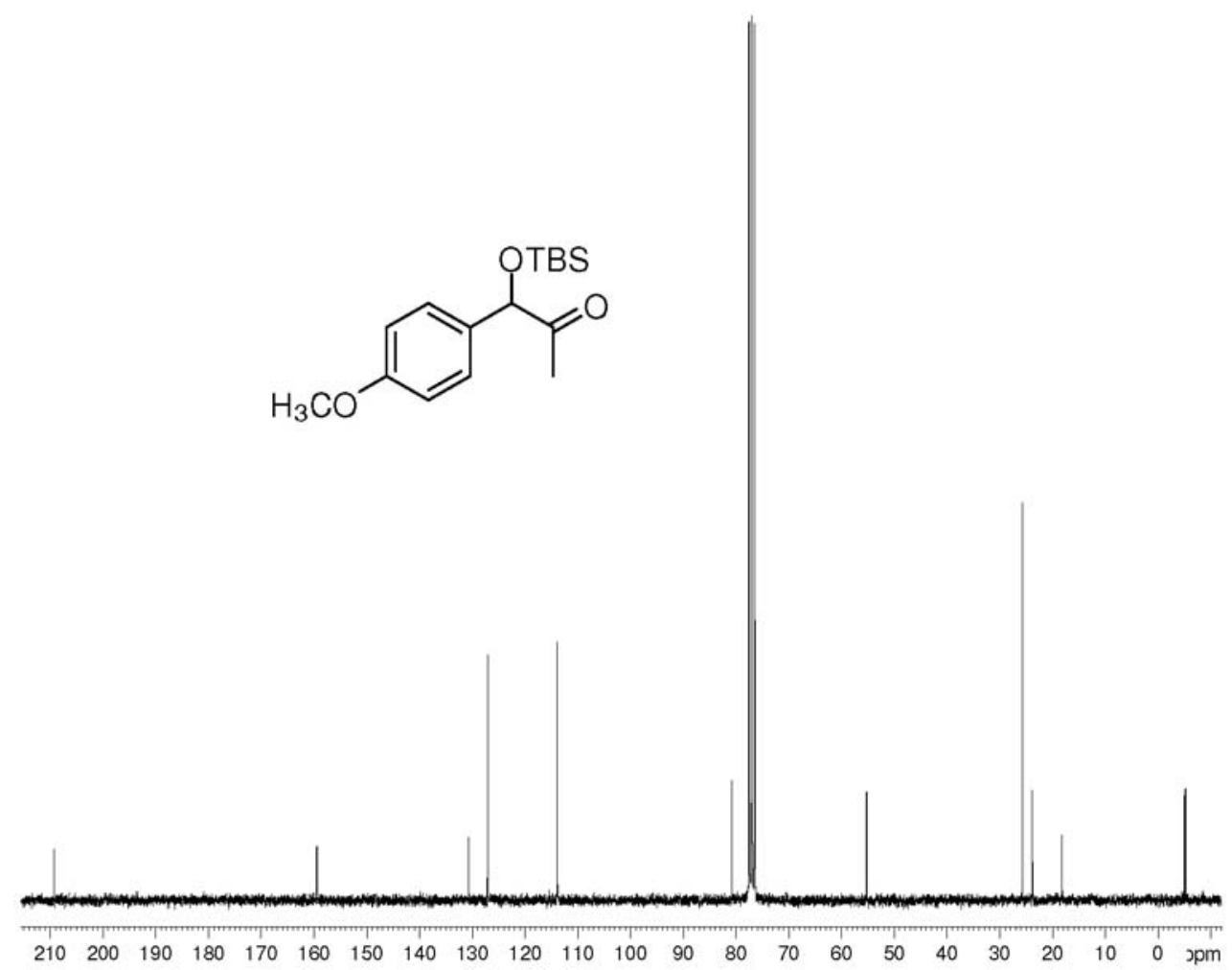

Figure S68. ${ }^{13} \mathrm{C} \mathrm{NMR}\left(\mathrm{CDCl}_{3}, 62.5 \mathrm{MHz}\right)$ of acyloin 36 .

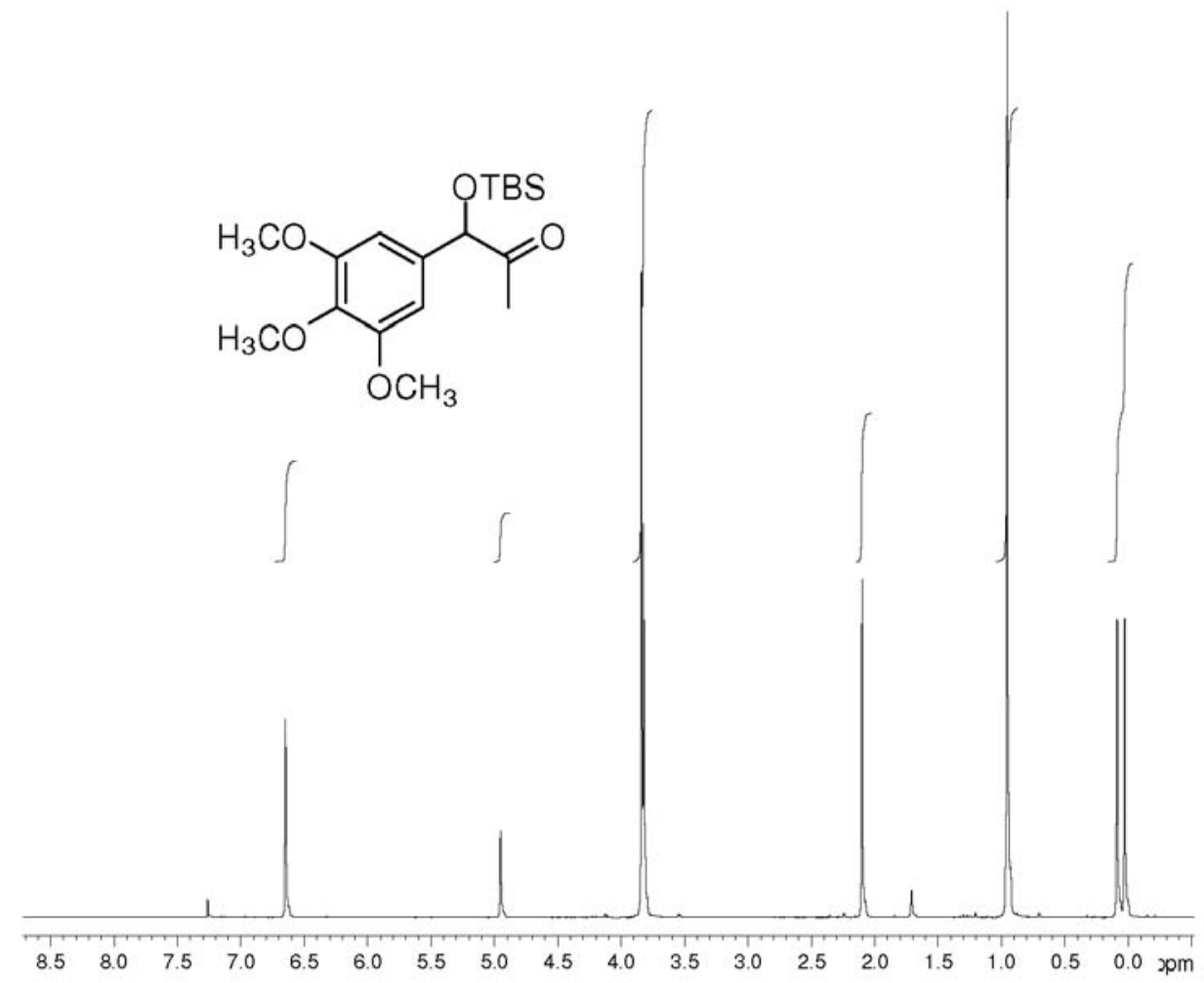

Figure S69. ${ }^{1} \mathrm{H}$ NMR $\left(\mathrm{CDCl}_{3}, 250 \mathrm{MHz}\right)$ of acyloin 37. 


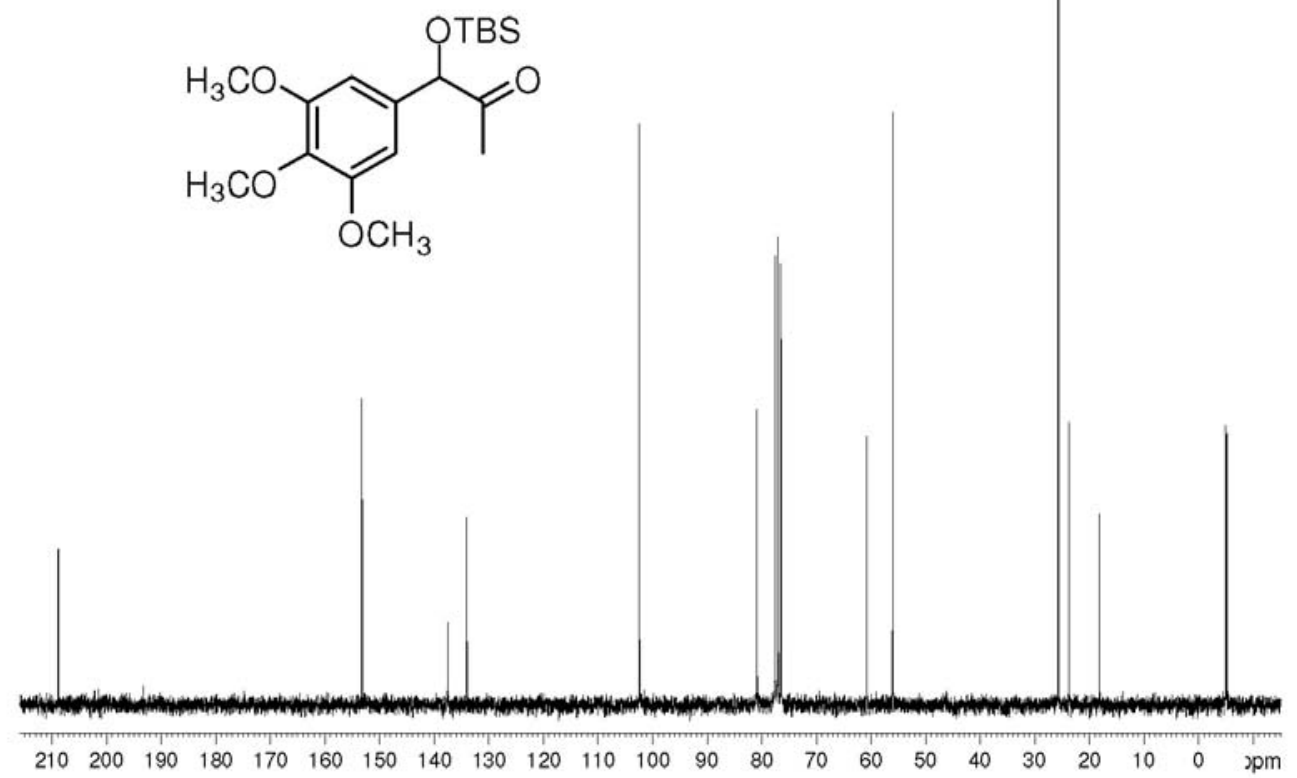

Figure S70. ${ }^{13} \mathrm{C}$ NMR $\left(\mathrm{CDCl}_{3}, 62.5 \mathrm{MHz}\right)$ of acyloin 37.

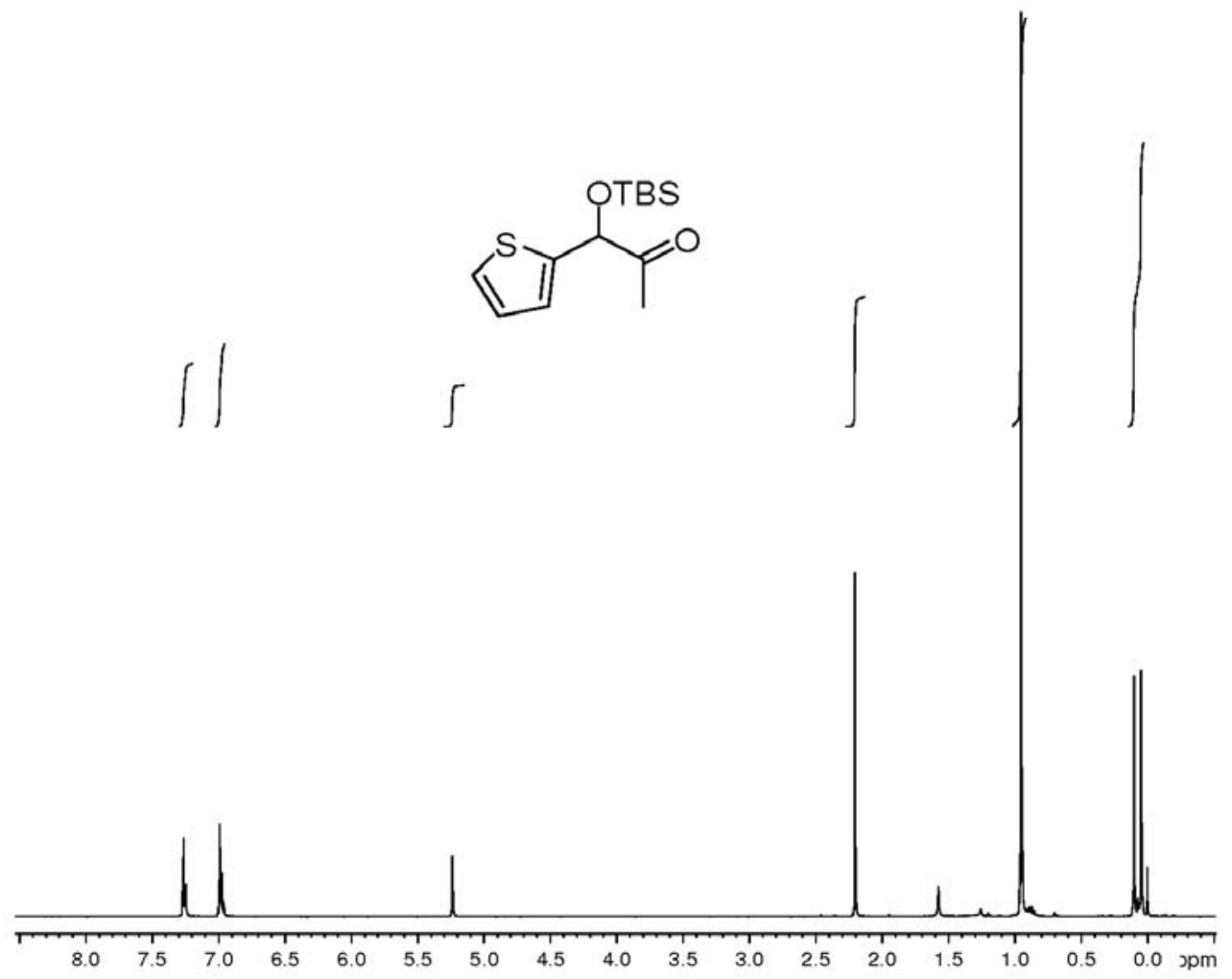

Figure S71. ${ }^{1} \mathrm{H} \mathrm{NMR}\left(\mathrm{CDCl}_{3}, 250 \mathrm{MHz}\right)$ of acyloin 38. 


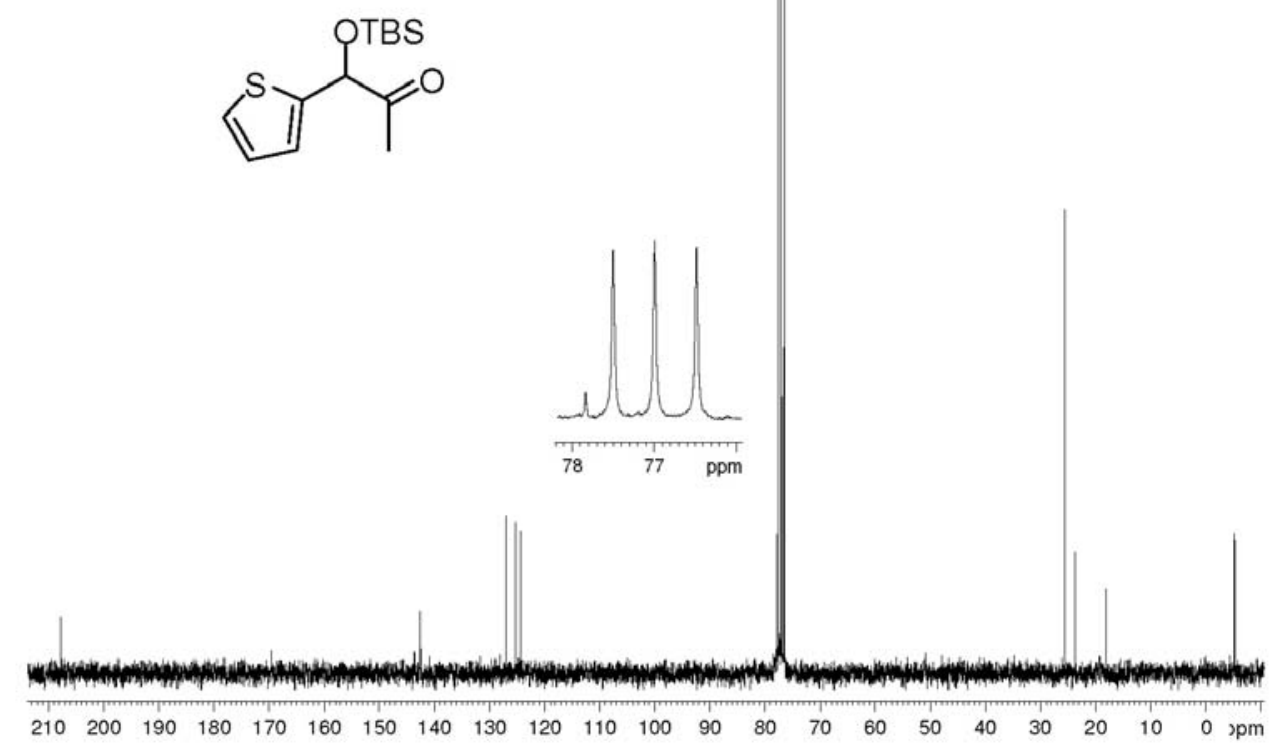

Figure S72. ${ }^{13} \mathrm{C}$ NMR $\left(\mathrm{CDCl}_{3}, 62.5 \mathrm{MHz}\right)$ of acyloin 38 .

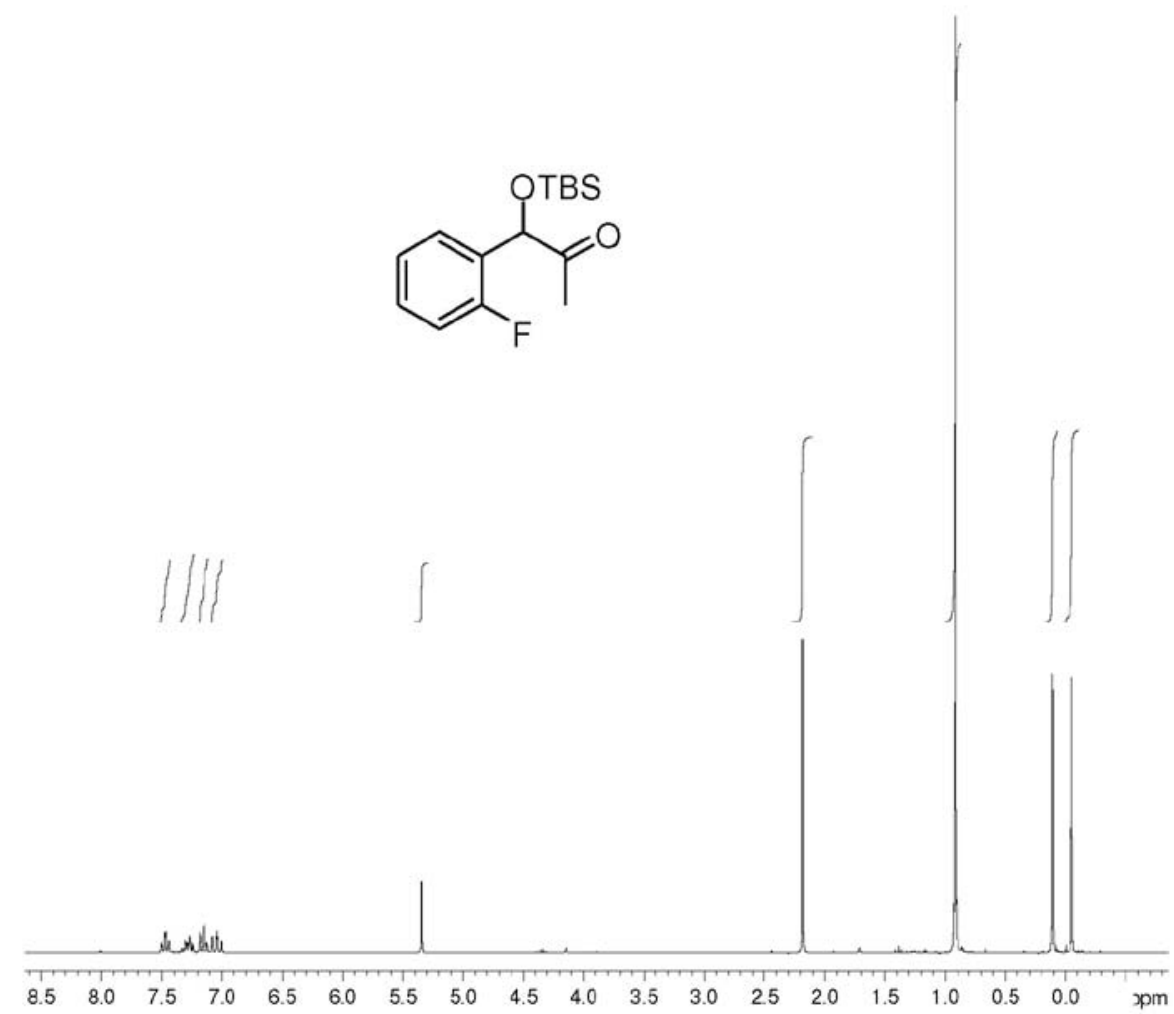

Figure S73. ${ }^{1} \mathrm{H}$ NMR $\left(\mathrm{CDCl}_{3}, 250 \mathrm{MHz}\right)$ of acyloin 39. 


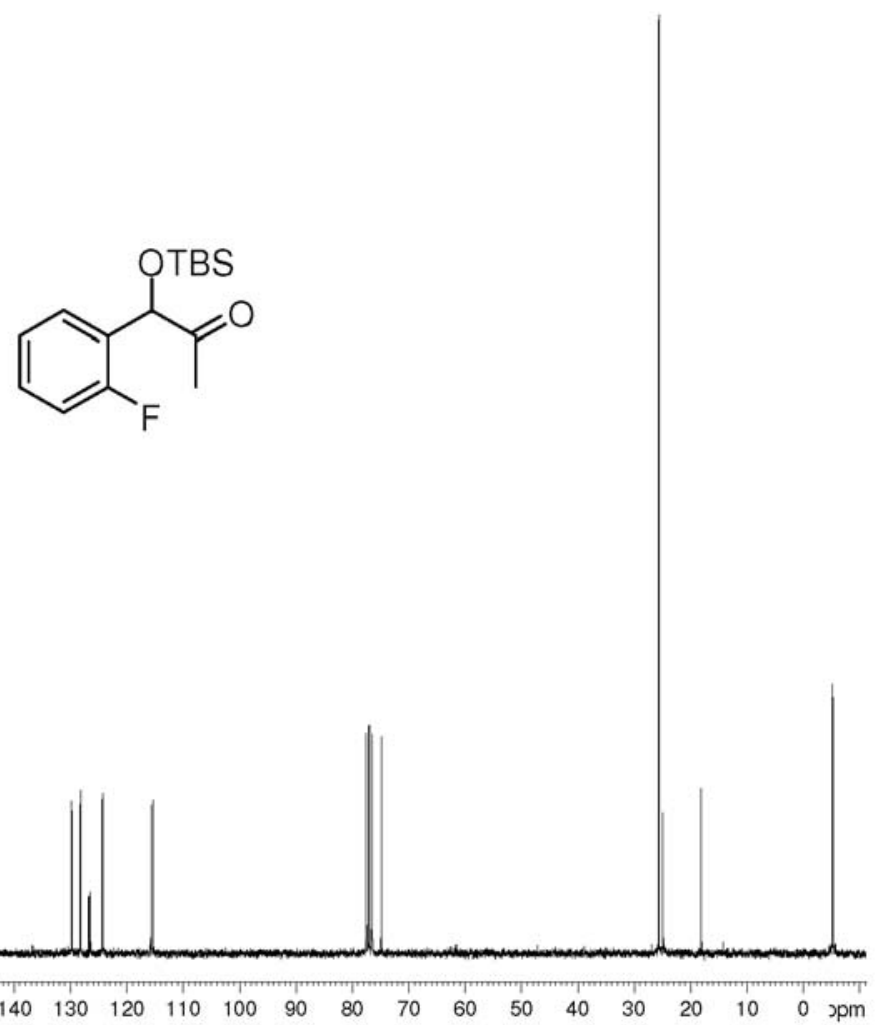

Figure S74. ${ }^{13} \mathrm{C}$ NMR $\left(\mathrm{CDCl}_{3}, 62.5 \mathrm{MHz}\right)$ of acyloin 39.

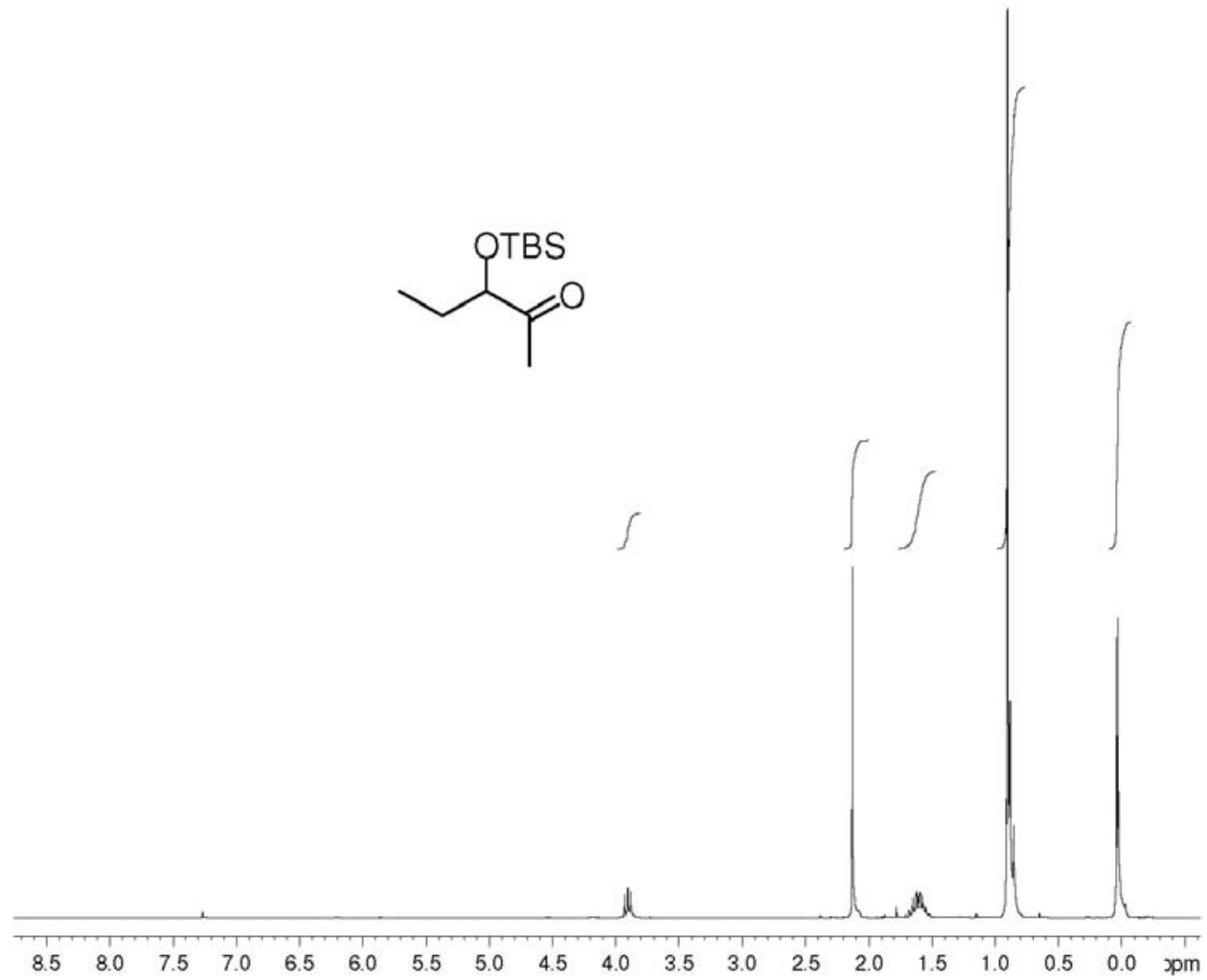

Figure S75. ${ }^{1} \mathrm{H}$ NMR $\left(\mathrm{CDCl}_{3}, 250 \mathrm{MHz}\right)$ of acyloin 40. 


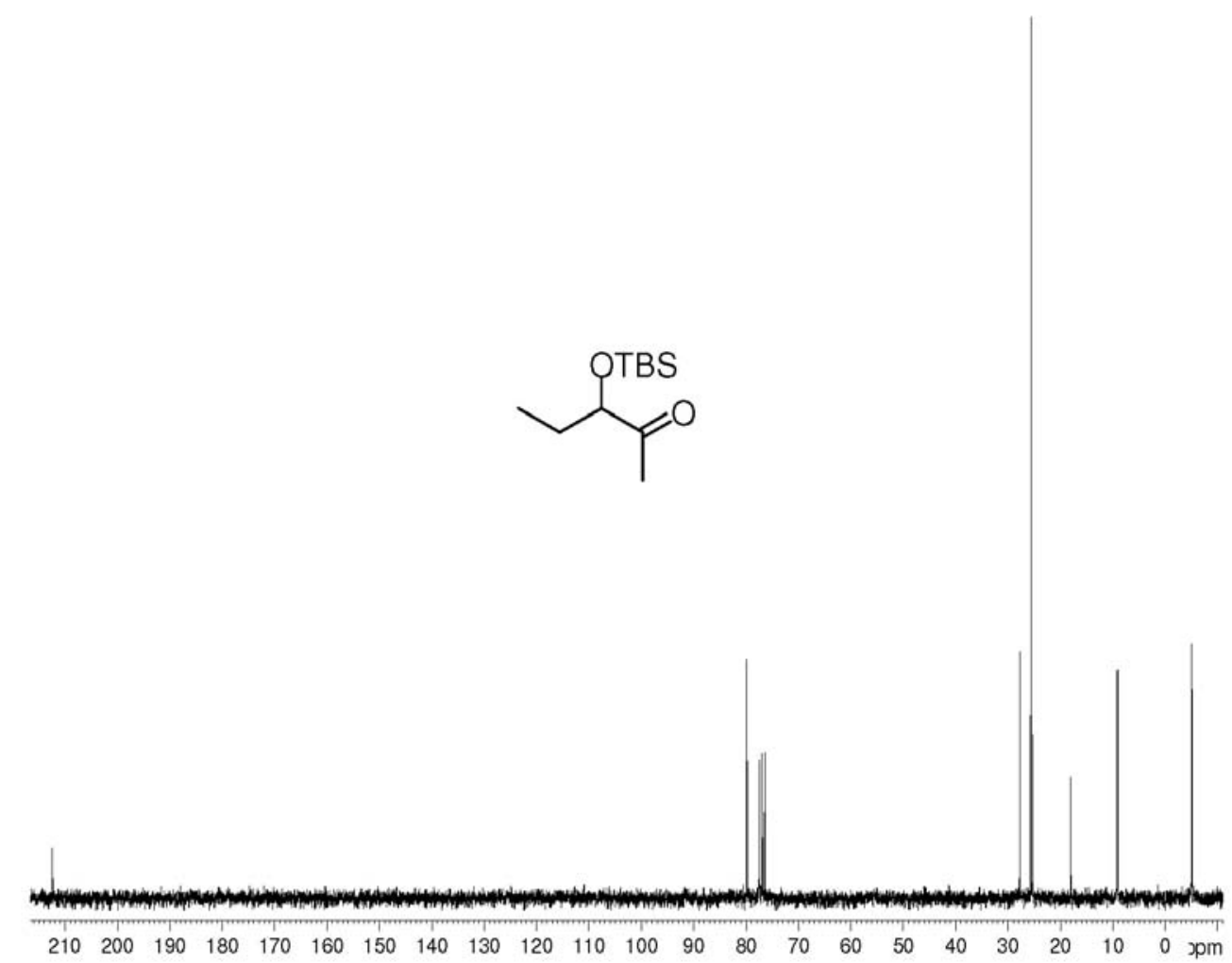

Figure S76. ${ }^{13} \mathrm{C}$ NMR $\left(\mathrm{CDCl}_{3}, 62.5 \mathrm{MHz}\right)$ of acyloin 40.

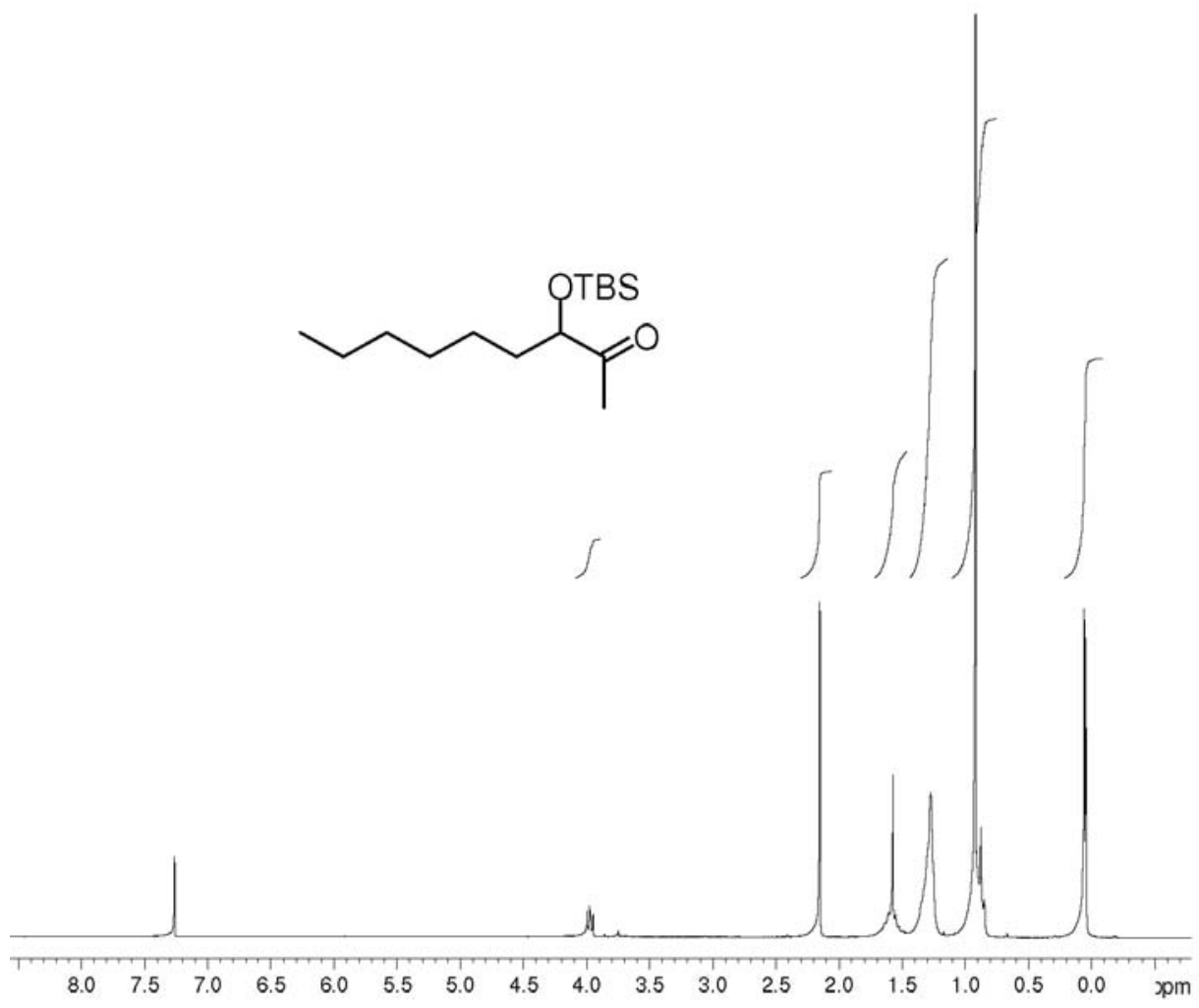

Figure S77. ${ }^{1} \mathrm{H}$ NMR $\left(\mathrm{CDCl}_{3}, 250 \mathrm{MHz}\right)$ of acyloin 41. 


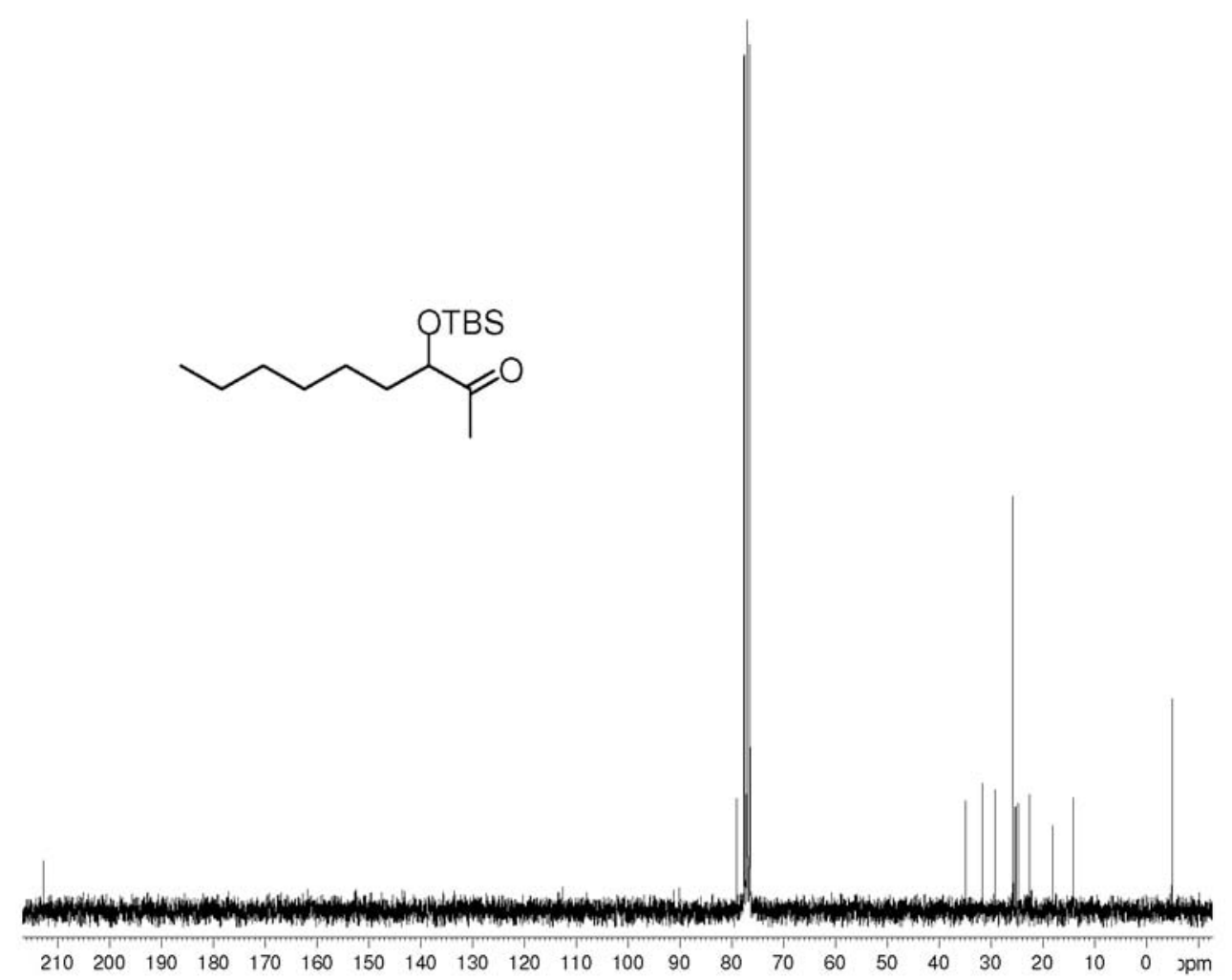

Figure S78. ${ }^{13} \mathrm{C}$ NMR $\left(\mathrm{CDCl}_{3}, 62.5 \mathrm{MHz}\right)$ of acyloin 41.

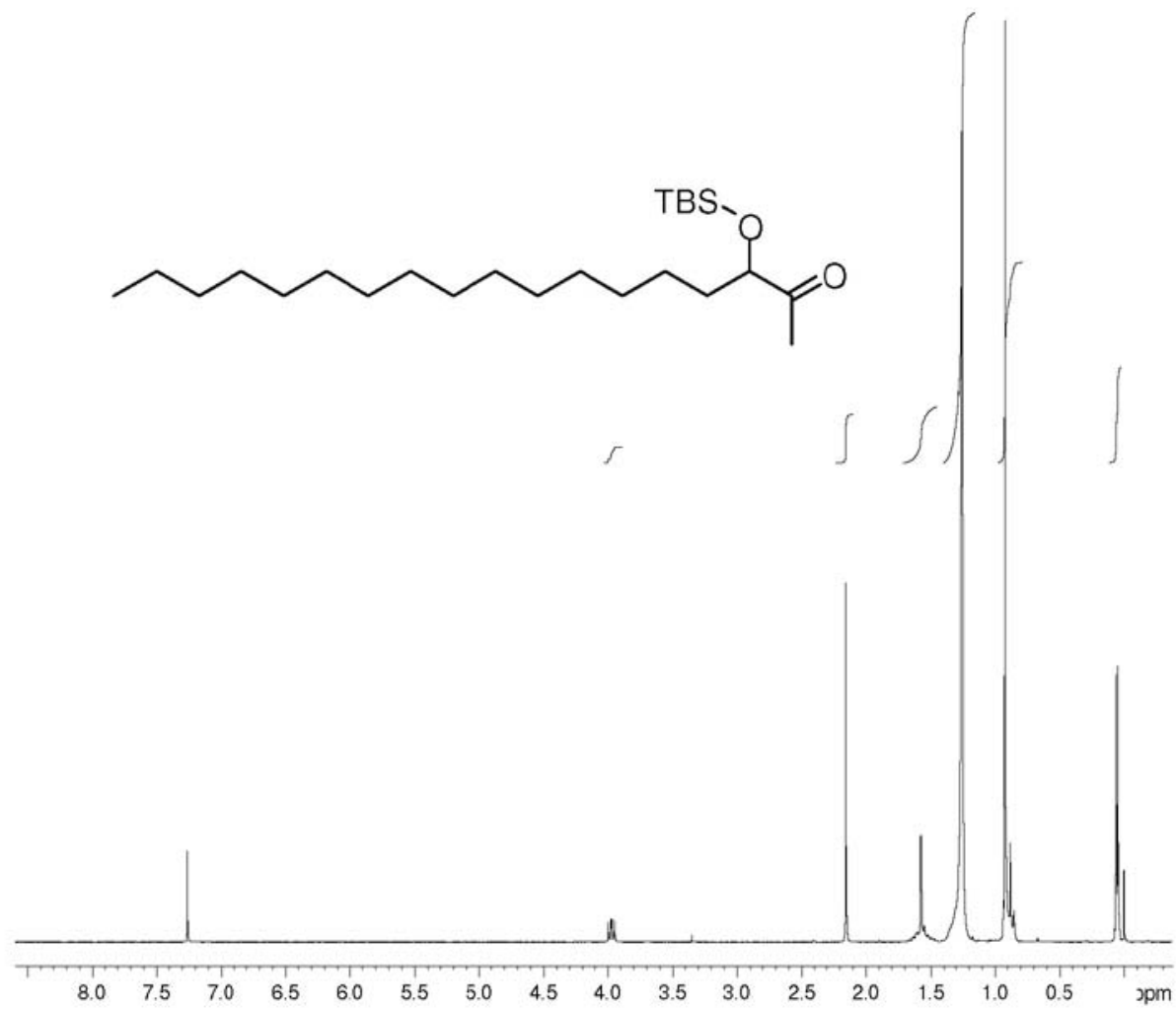

Figure S79. ${ }^{1} \mathrm{H}$ NMR $\left(\mathrm{CDCl}_{3}, 250 \mathrm{MHz}\right)$ of acyloin 42. 


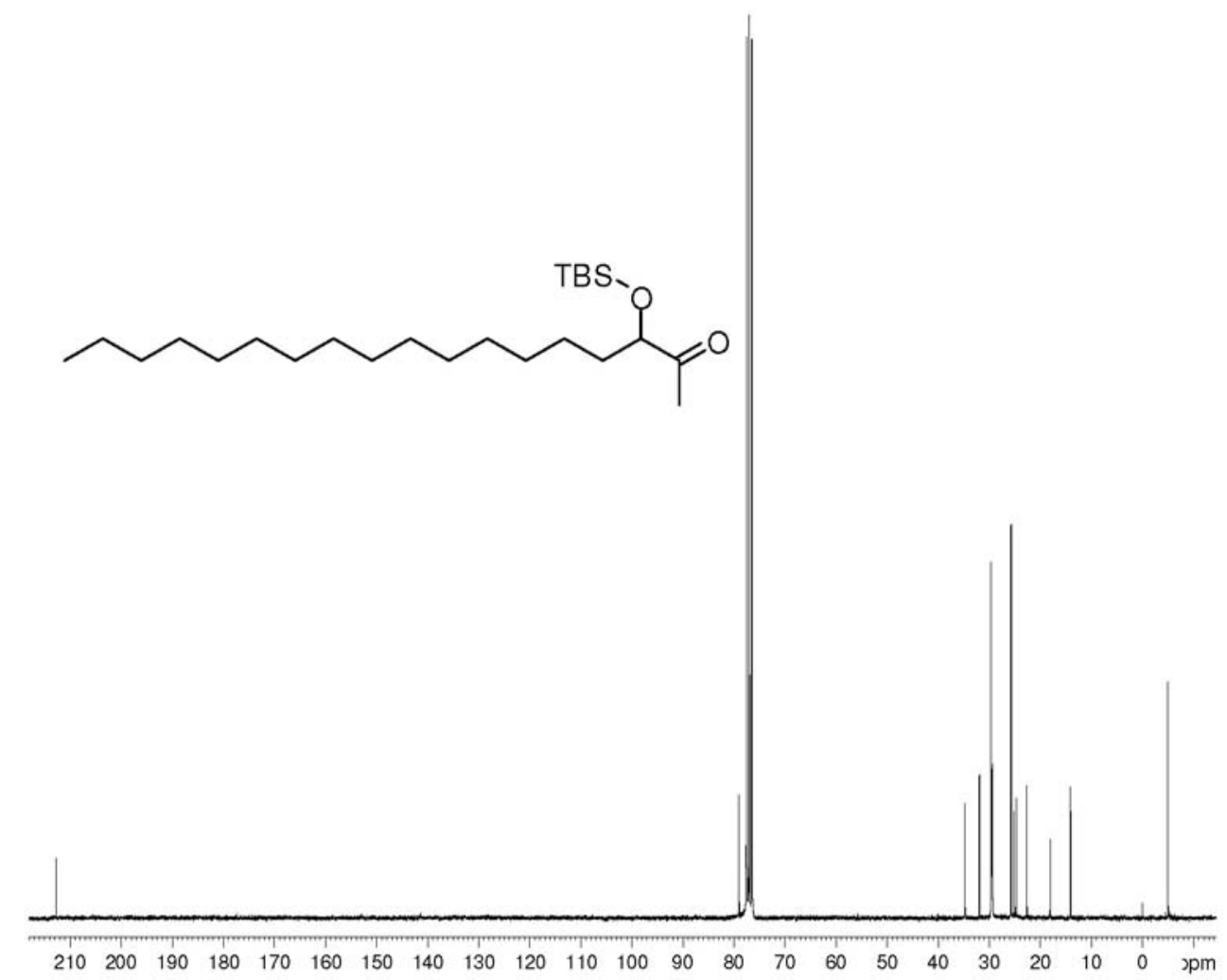

Figure S80. ${ }^{13} \mathrm{C} \mathrm{NMR}\left(\mathrm{CDCl}_{3}, 62.5 \mathrm{MHz}\right)$ of acyloin 41.

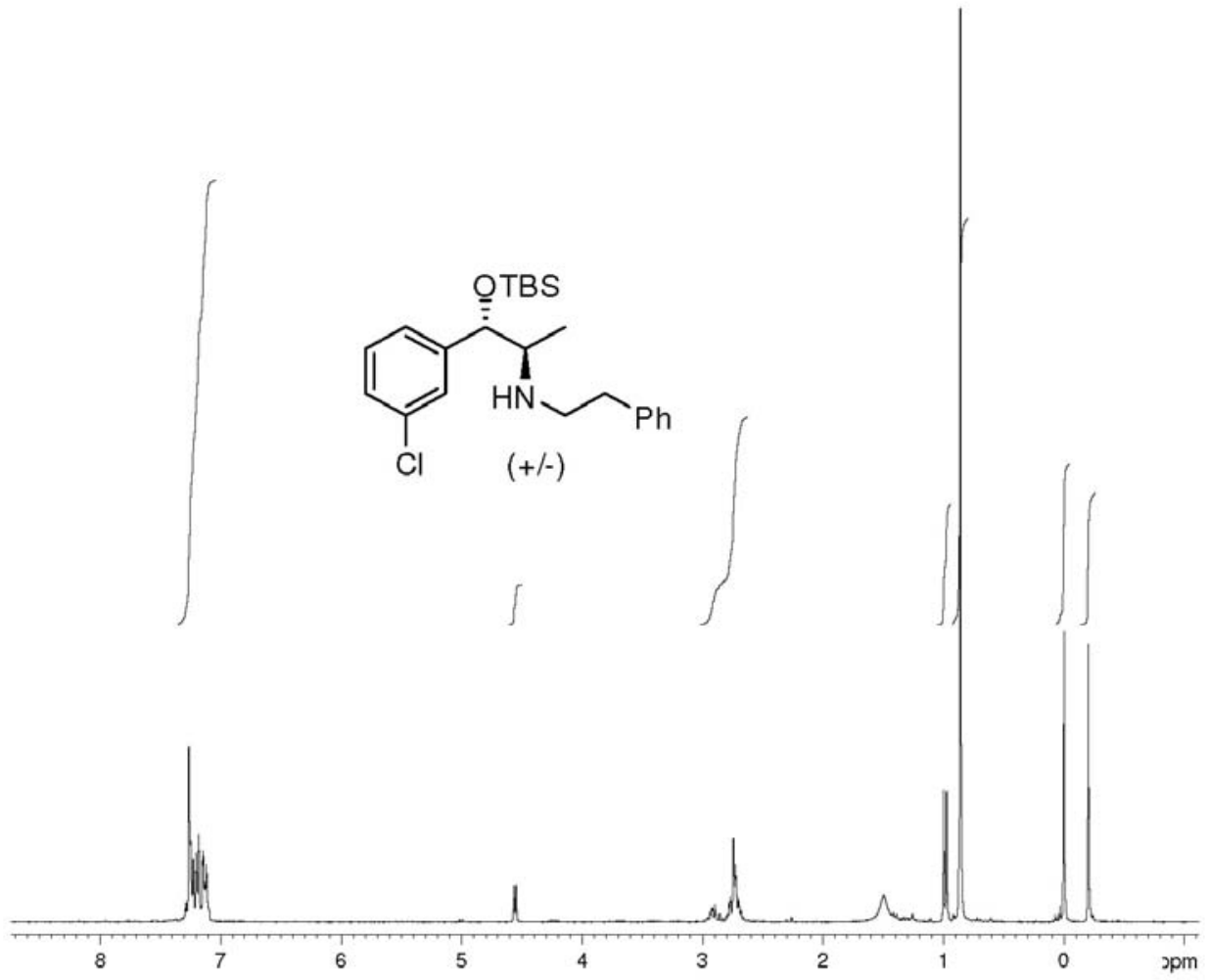

Figure S81. ${ }^{1} \mathrm{H}$ NMR $\left(\mathrm{CDCl}_{3}, 250 \mathrm{MHz}\right)$ of vicinal aminoalcohol 43. 

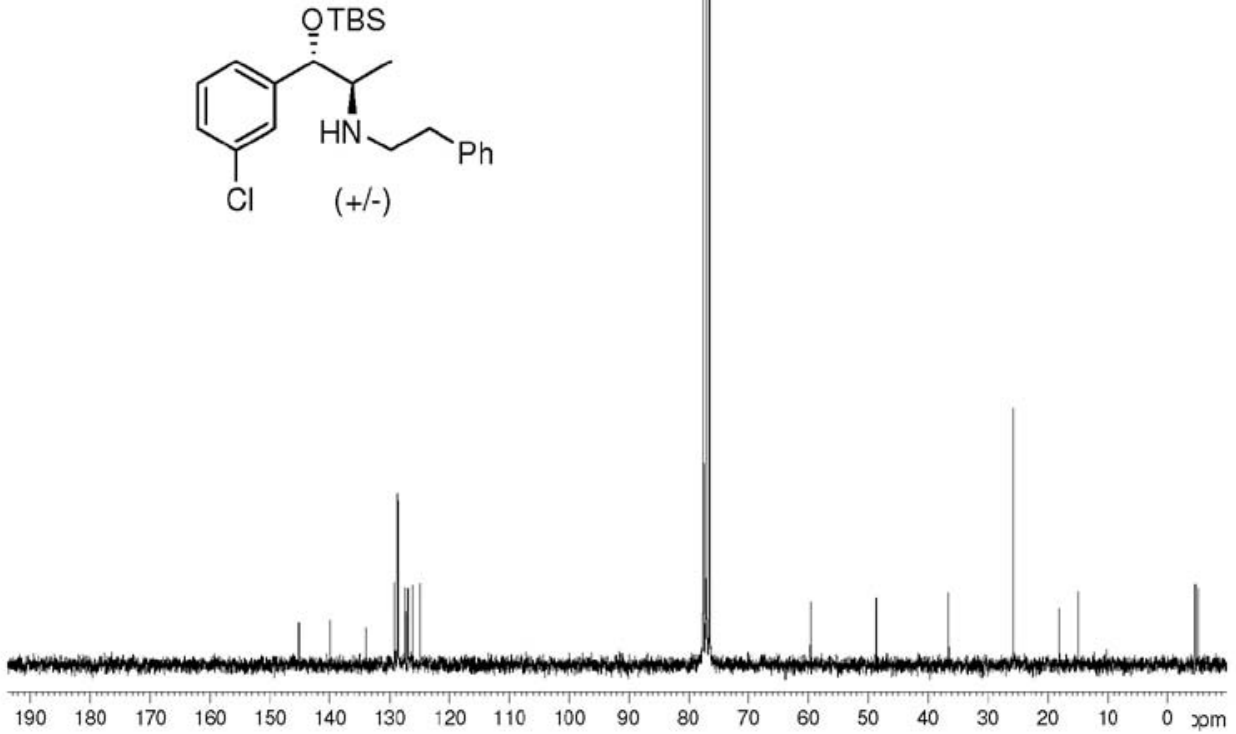

Figure S82. ${ }^{13} \mathrm{C}$ NMR $\left(\mathrm{CDCl}_{3}, 62.5 \mathrm{MHz}\right)$ of vicinal aminoalcohol 43.
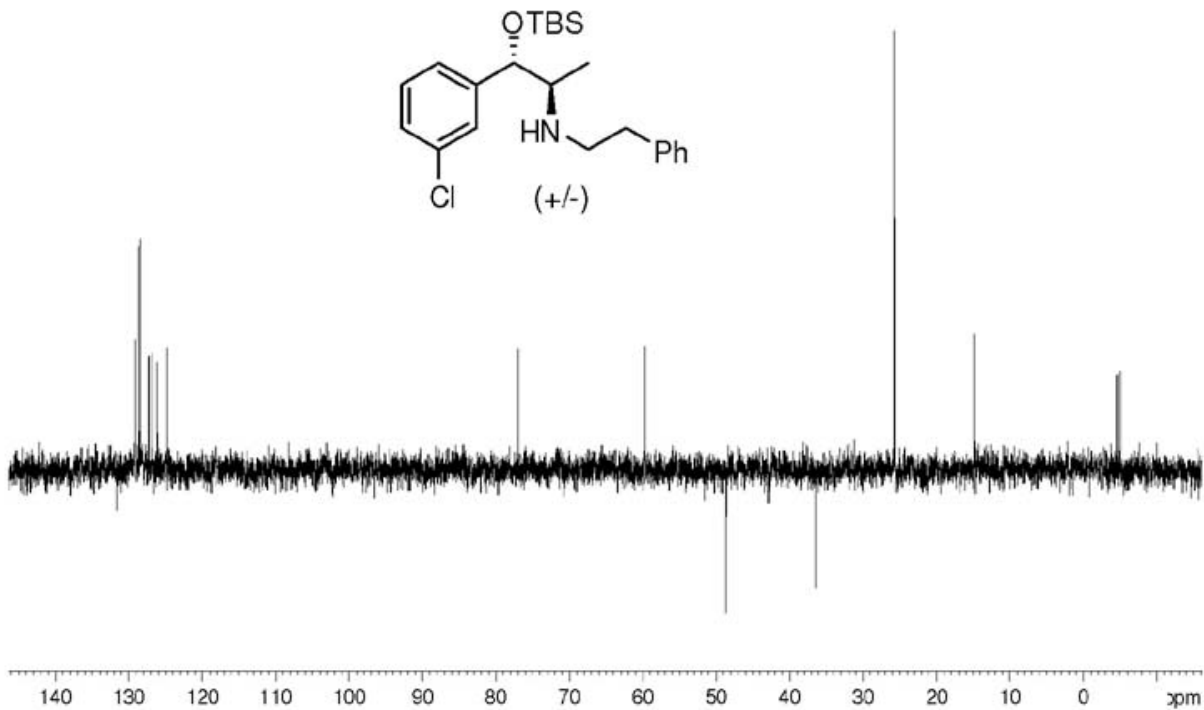

Figure S83. DEPT $135\left(\mathrm{CDCl}_{3}, 62.5 \mathrm{MHz}\right)$ of of vicinal aminoalcohol 43. 


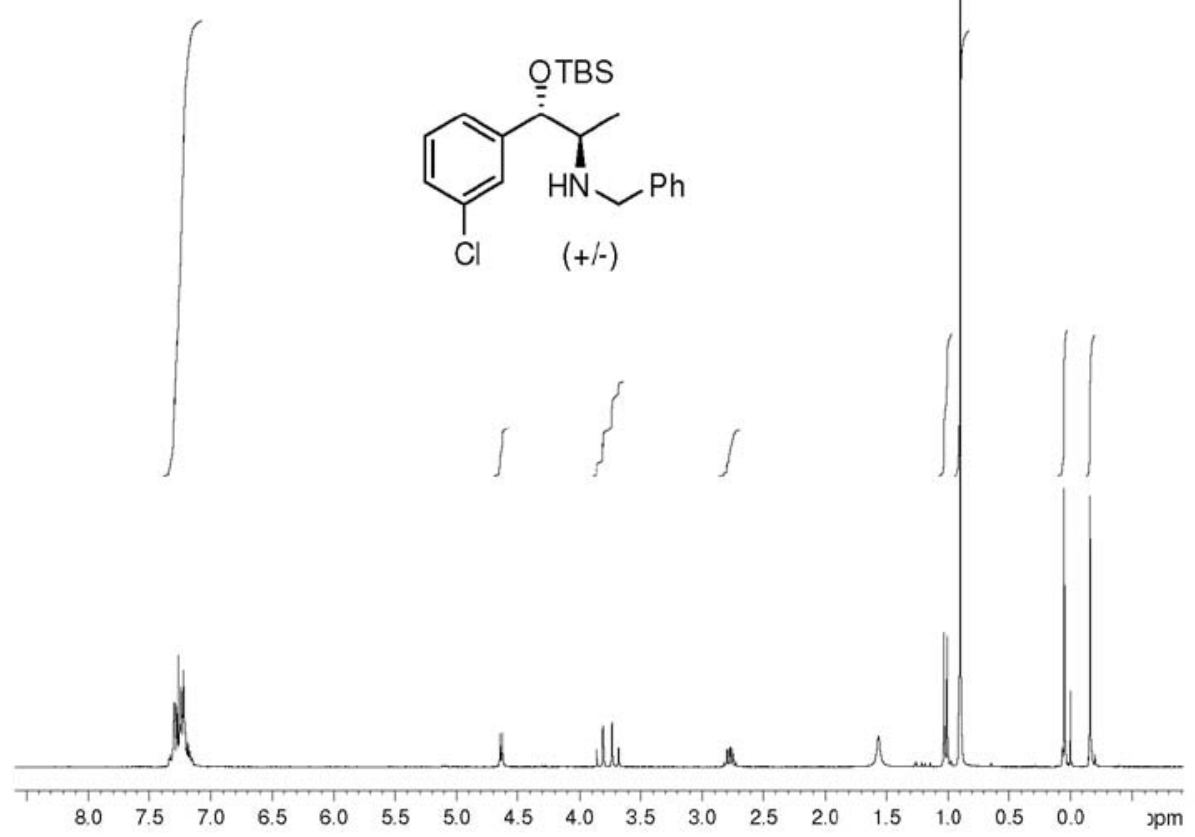

Figure S84. ${ }^{1} \mathrm{H} \mathrm{NMR}\left(\mathrm{CDCl}_{3}, 250 \mathrm{MHz}\right)$ of vicinal aminoalcohol 44.

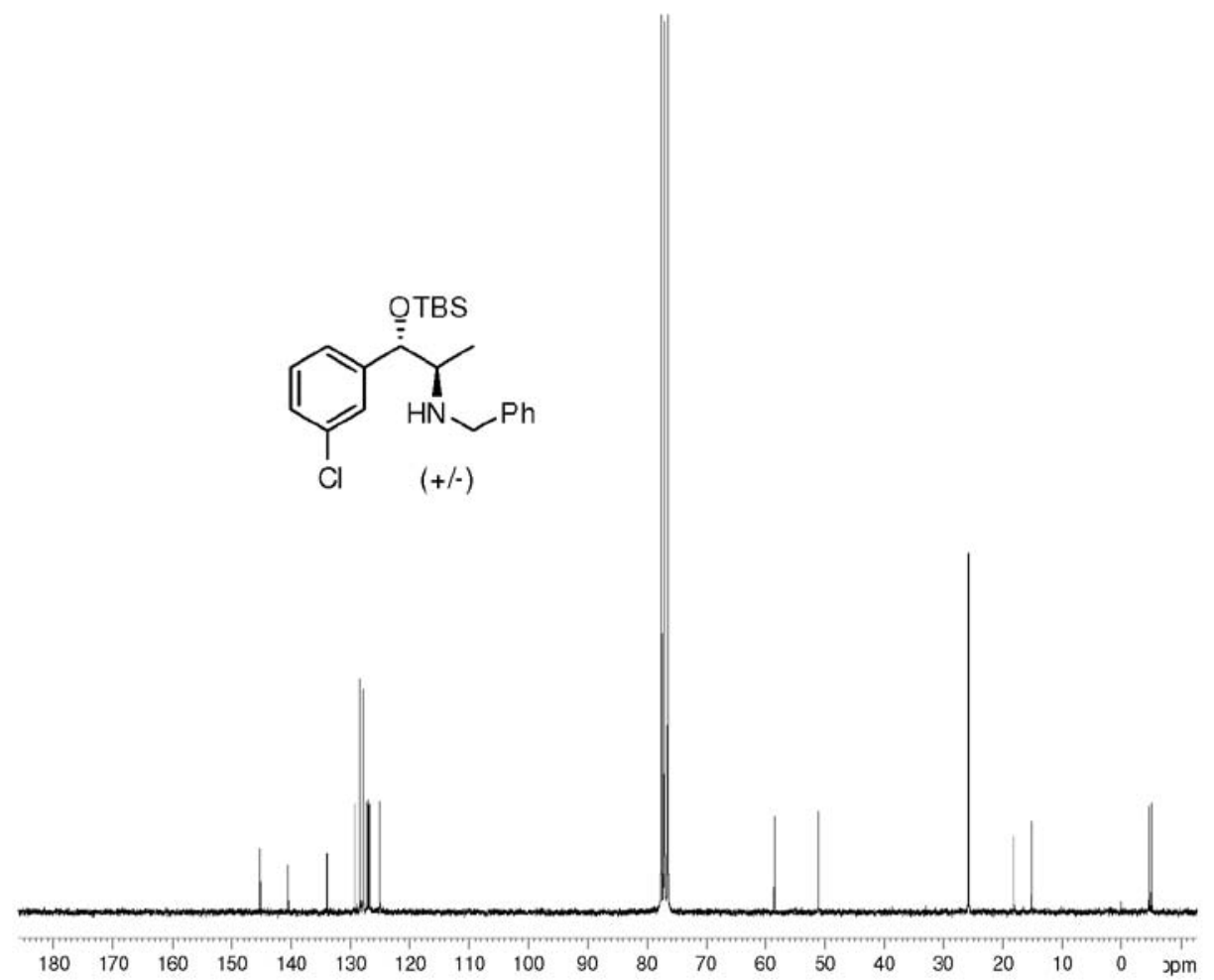

Figure S85. ${ }^{13} \mathrm{C}$ NMR $\left(\mathrm{CDCl}_{3}, 62.5 \mathrm{MHz}\right)$ of vicinal aminoalcohol 44. 


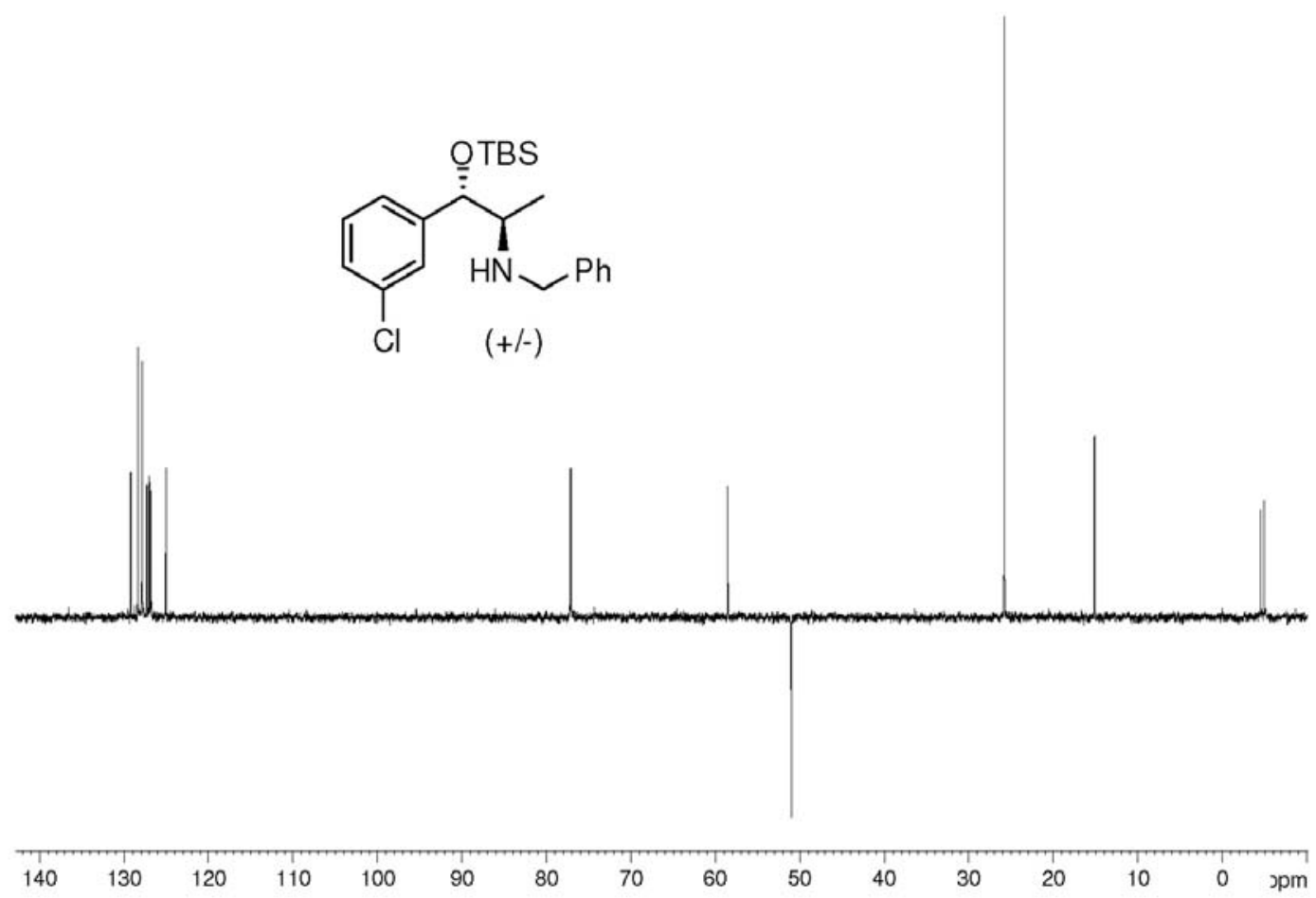

Figure S86. DEPT $135\left(\mathrm{CDCl}_{3}, 62.5 \mathrm{MHz}\right)$ of vicinal aminoalcohol 44.

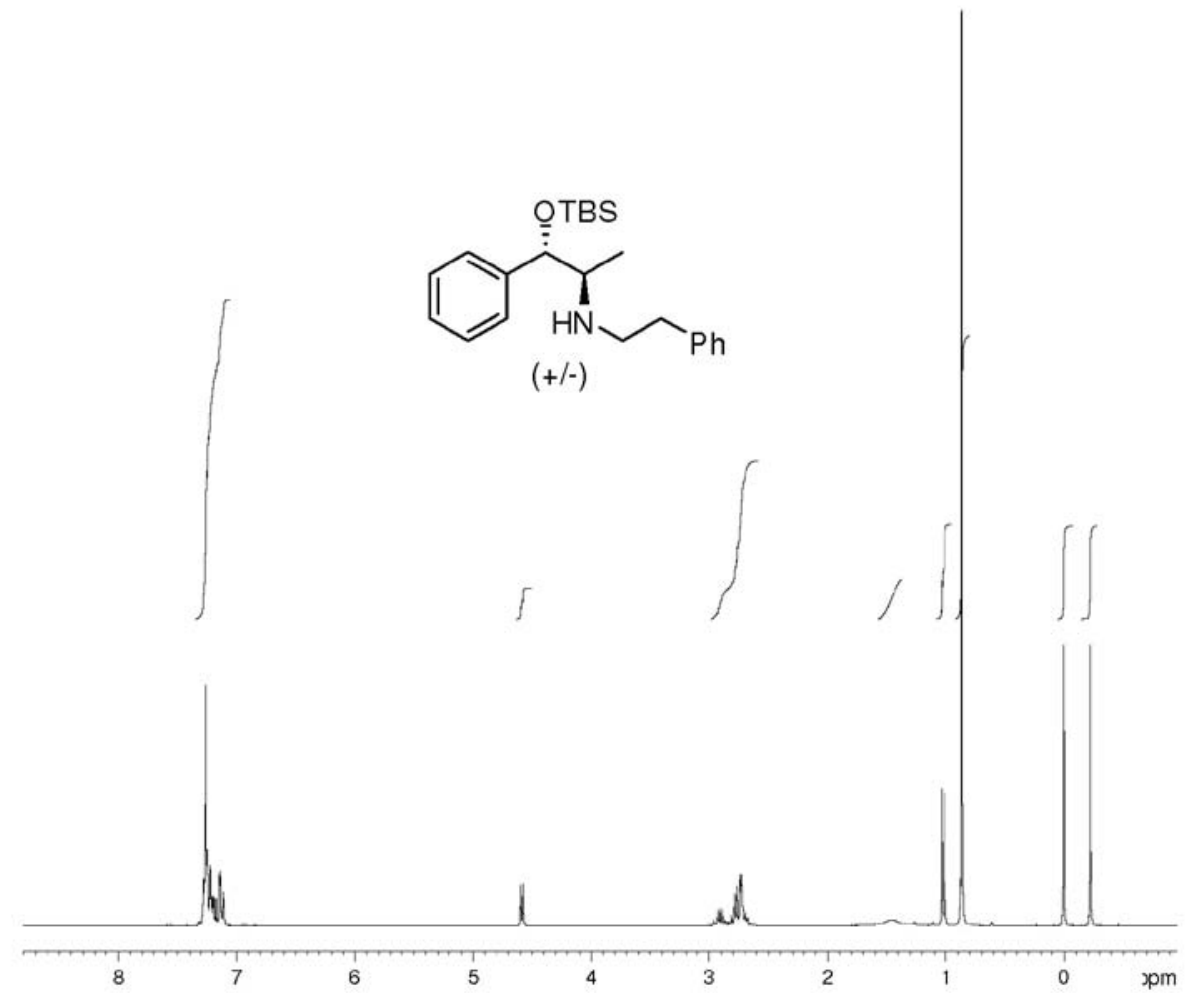

Figure S87. ${ }^{1} \mathrm{H}$ NMR $\left(\mathrm{CDCl}_{3}, 250 \mathrm{MHz}\right)$ of vicinal aminoalcohol 45. 


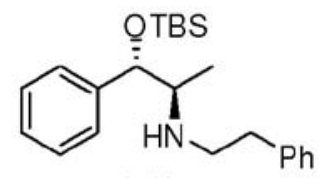

$(+/-)$

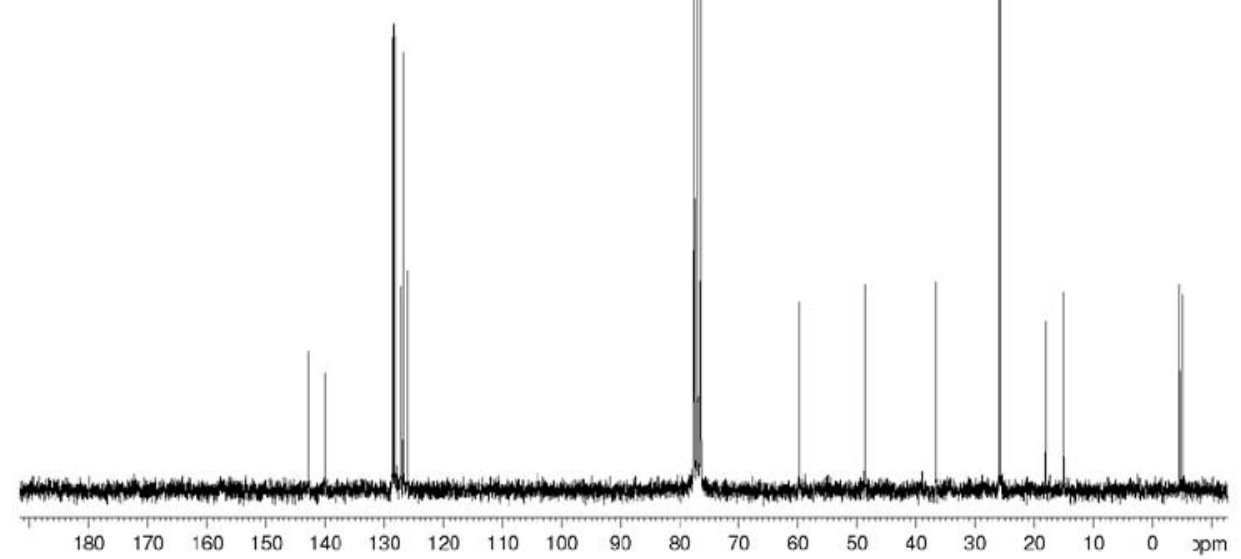

Figure S88. ${ }^{13} \mathrm{C}$ NMR $\left(\mathrm{CDCl}_{3}, 62.5 \mathrm{MHz}\right)$ of vicinal aminoalcohol 45.

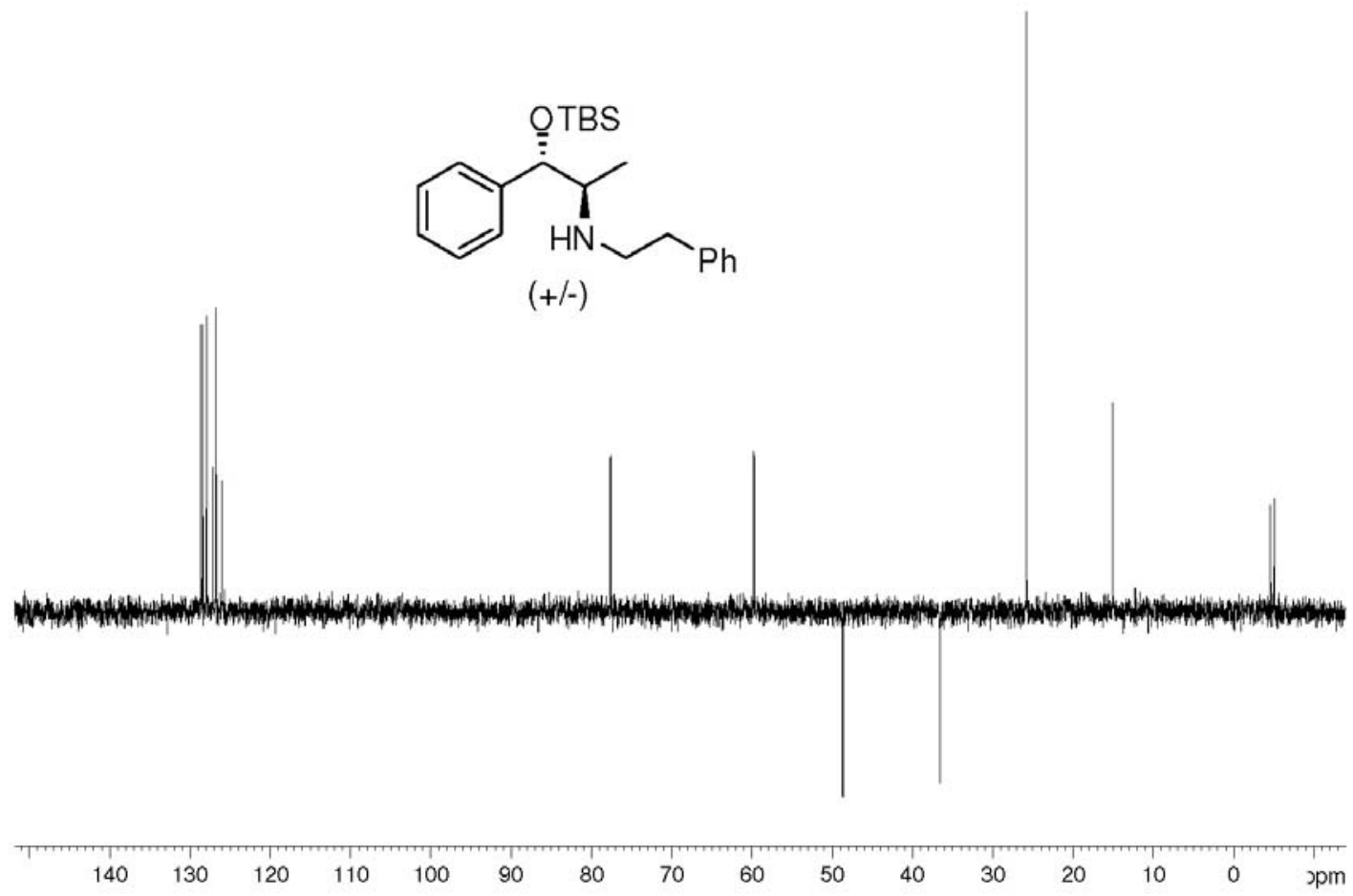

Figure S89. DEPT $135\left(\mathrm{CDCl}_{3}, 62.5 \mathrm{MHz}\right)$ of vicinal aminoalcohol 45. 


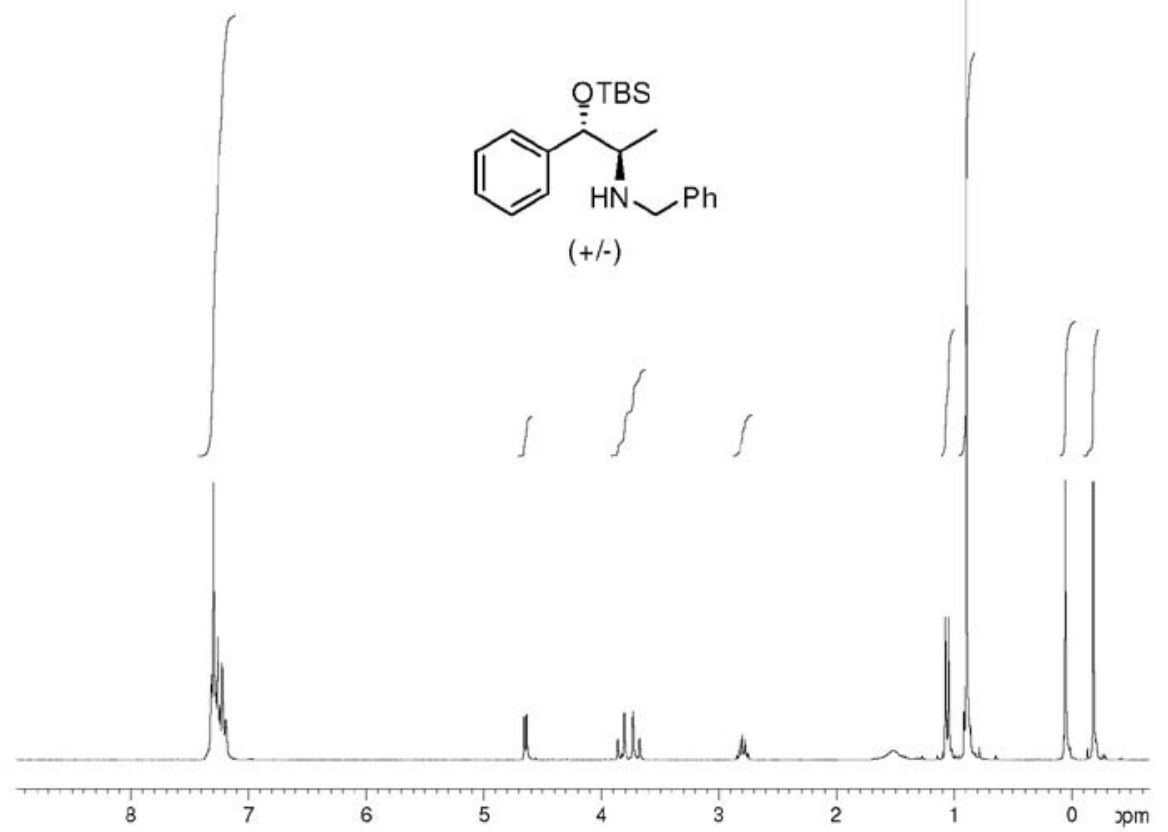

Figure S90. ${ }^{1} \mathrm{H} \mathrm{NMR}\left(\mathrm{CDCl}_{3}, 250 \mathrm{MHz}\right)$ of vicinal aminoalcohol 46.

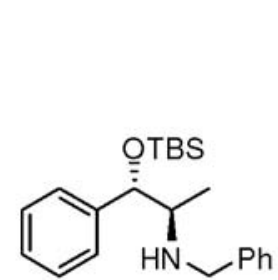

$(+/-)$

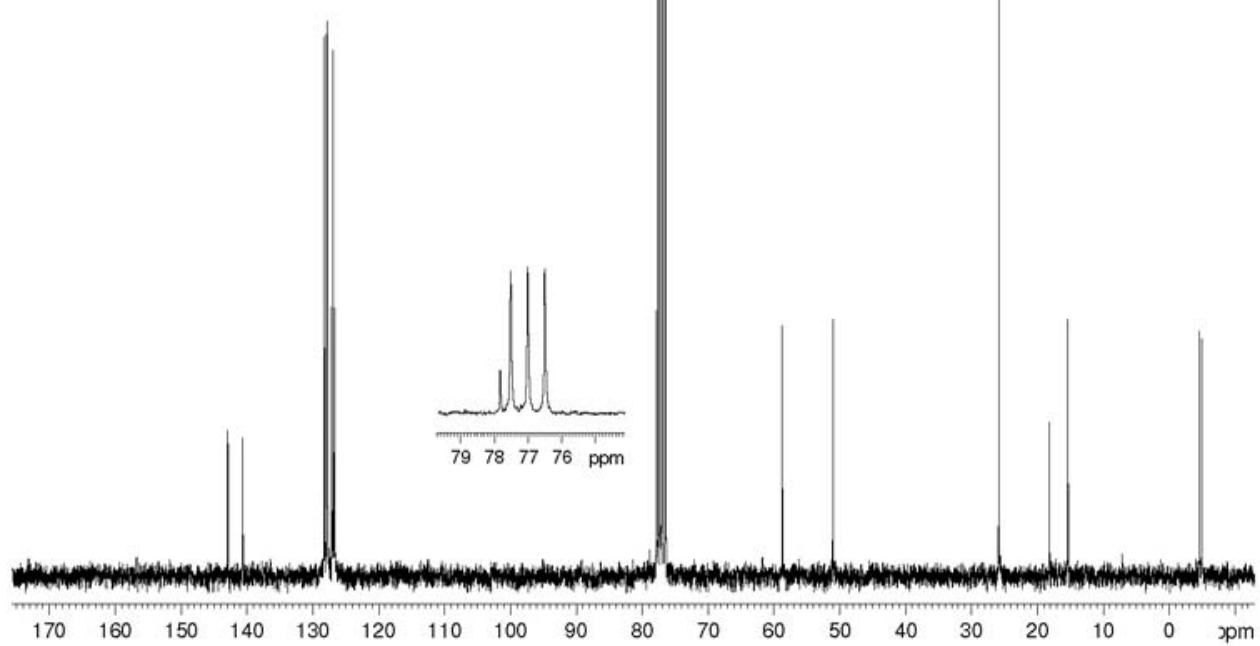

Figure S91. ${ }^{13} \mathrm{C}$ NMR $\left(\mathrm{CDCl}_{3}, 62.5 \mathrm{MHz}\right)$ of vicinal aminoalcohol 46. 


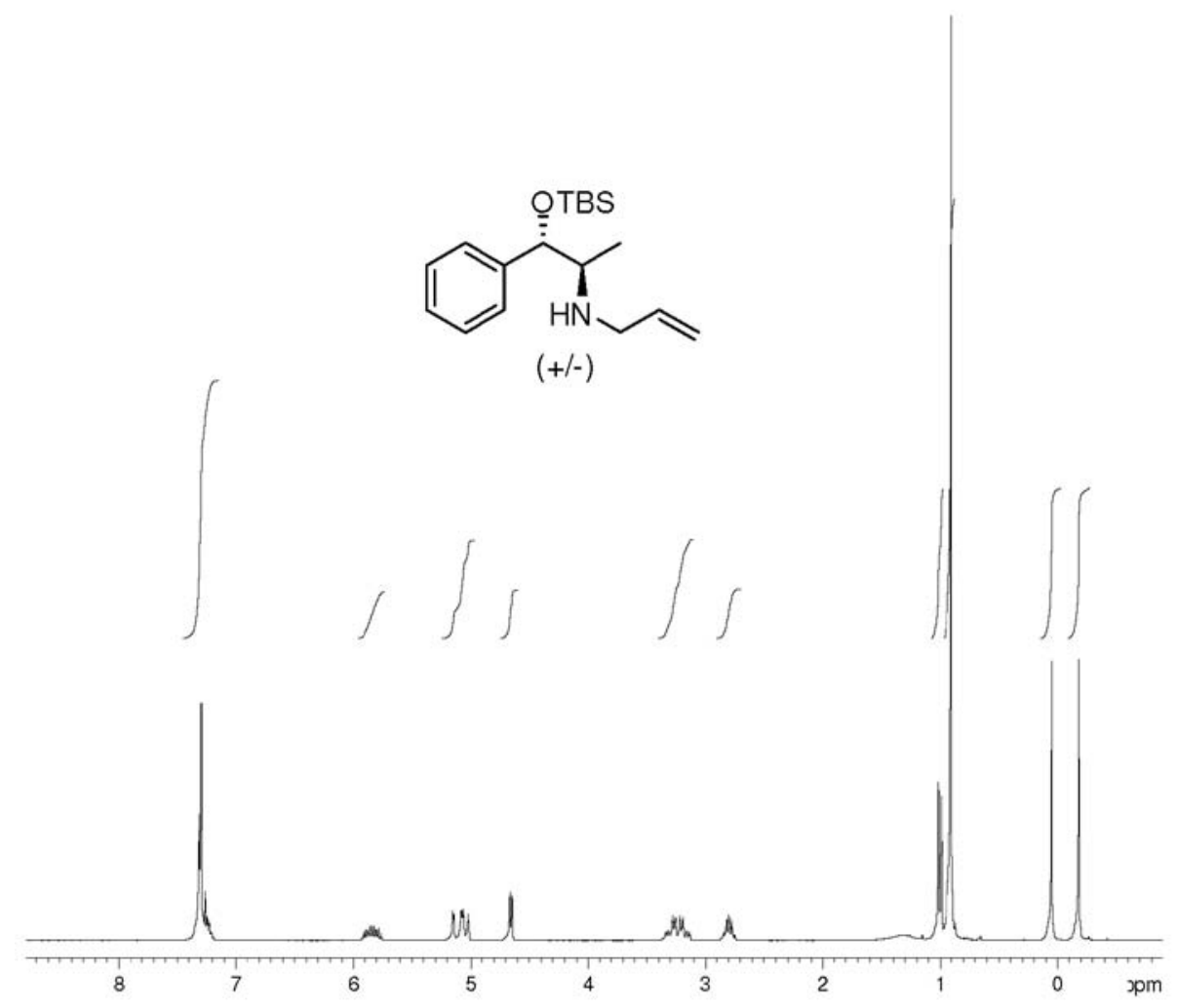

Figure S92. ${ }^{1} \mathrm{H} \mathrm{NMR}\left(\mathrm{CDCl}_{3}, 250 \mathrm{MHz}\right)$ of vicinal aminoalcohol 47.

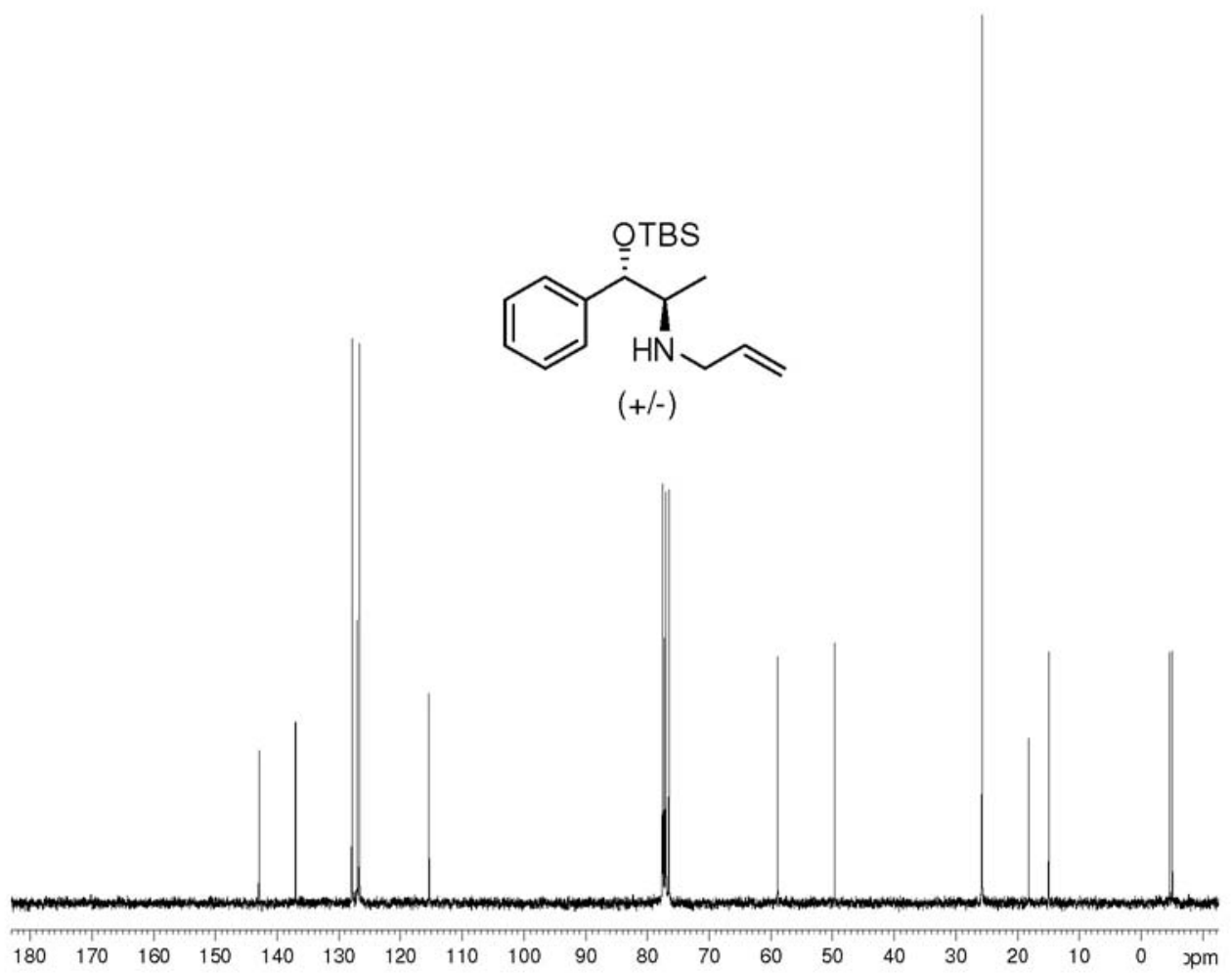

Figure S93. ${ }^{13} \mathrm{C}$ NMR $\left(\mathrm{CDCl}_{3}, 62.5 \mathrm{MHz}\right)$ of vicinal aminoalcohol 47. 


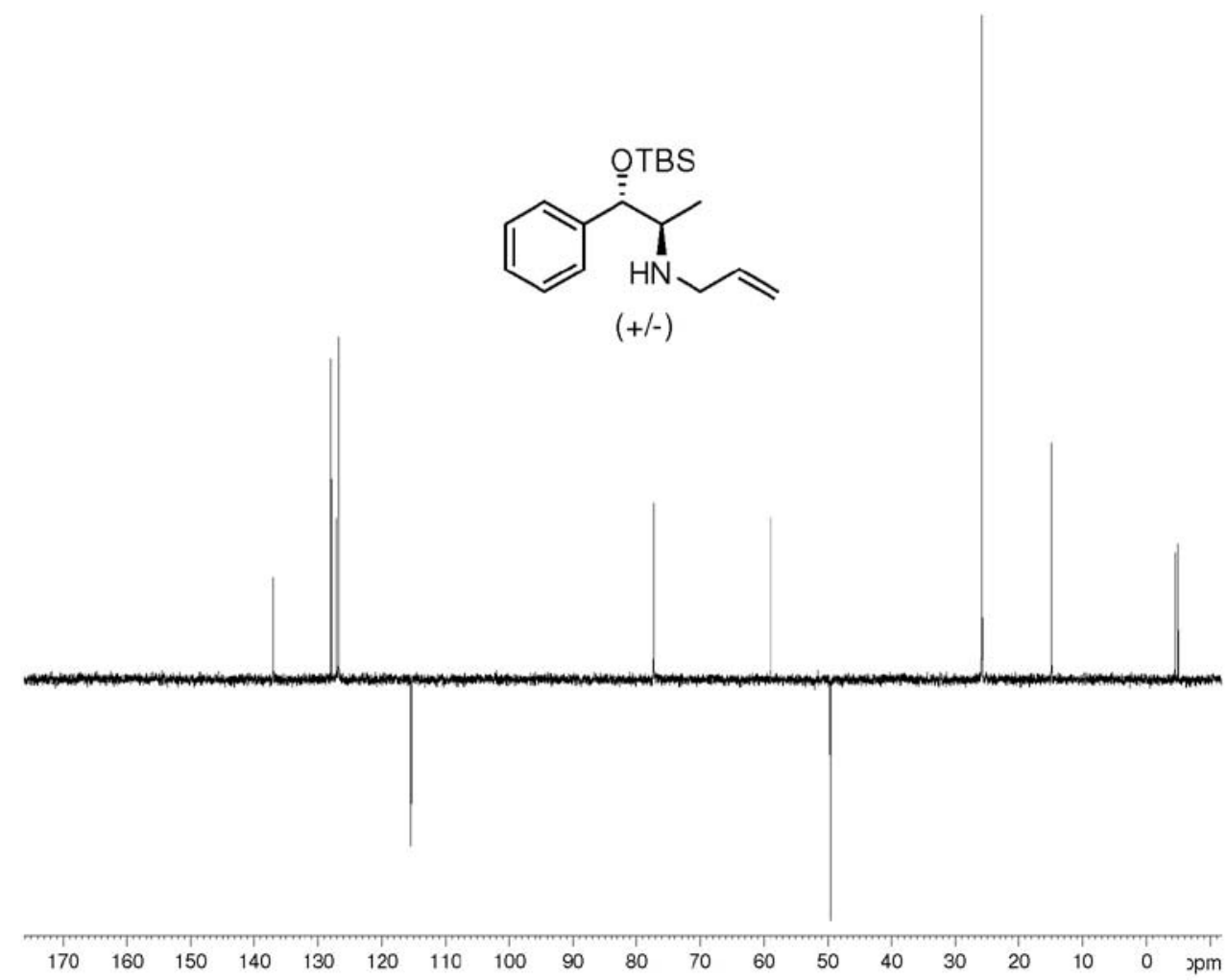

Figure S94. DEPT $135\left(\mathrm{CDCl}_{3}, 62.5 \mathrm{MHz}\right)$ of vicinal aminoalcohol 47.

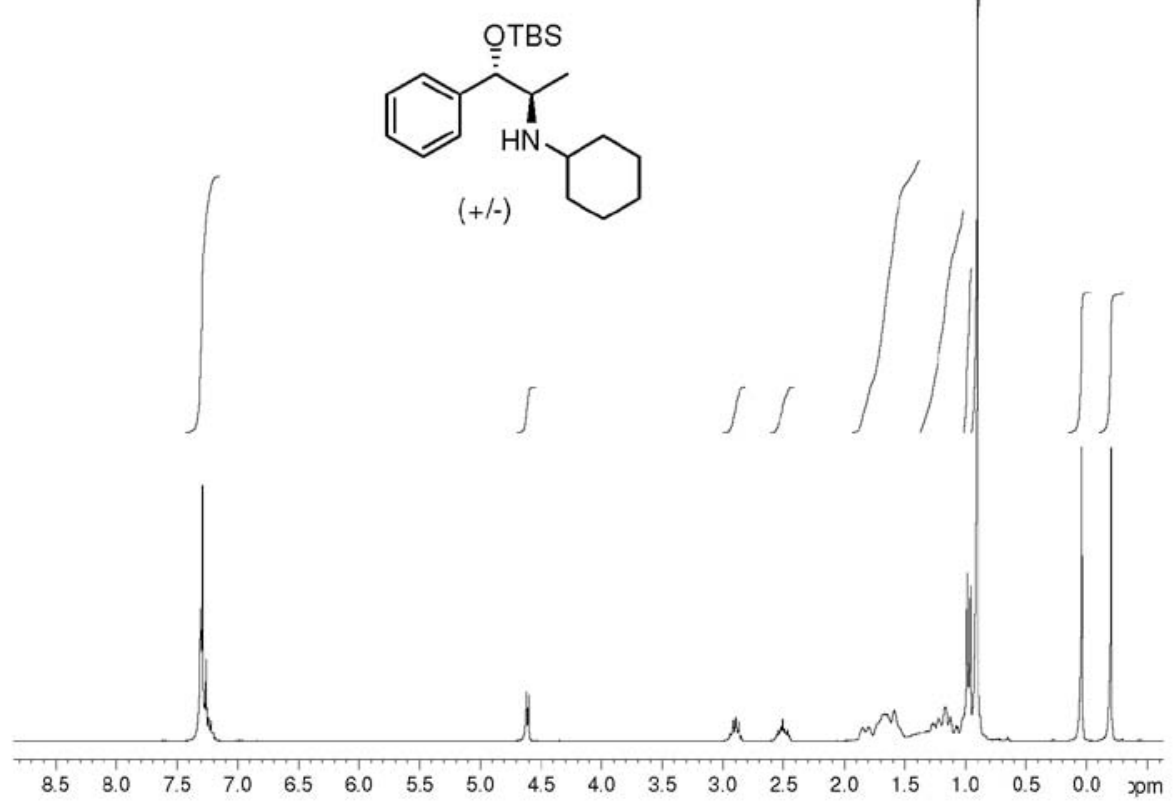

Figure S95. ${ }^{1} \mathrm{H}$ NMR $\left(\mathrm{CDCl}_{3}, 250 \mathrm{MHz}\right)$ of vicinal aminoalcohol 48. 
Vol. 22, No. 8, 2011

Amarante et al.

S49

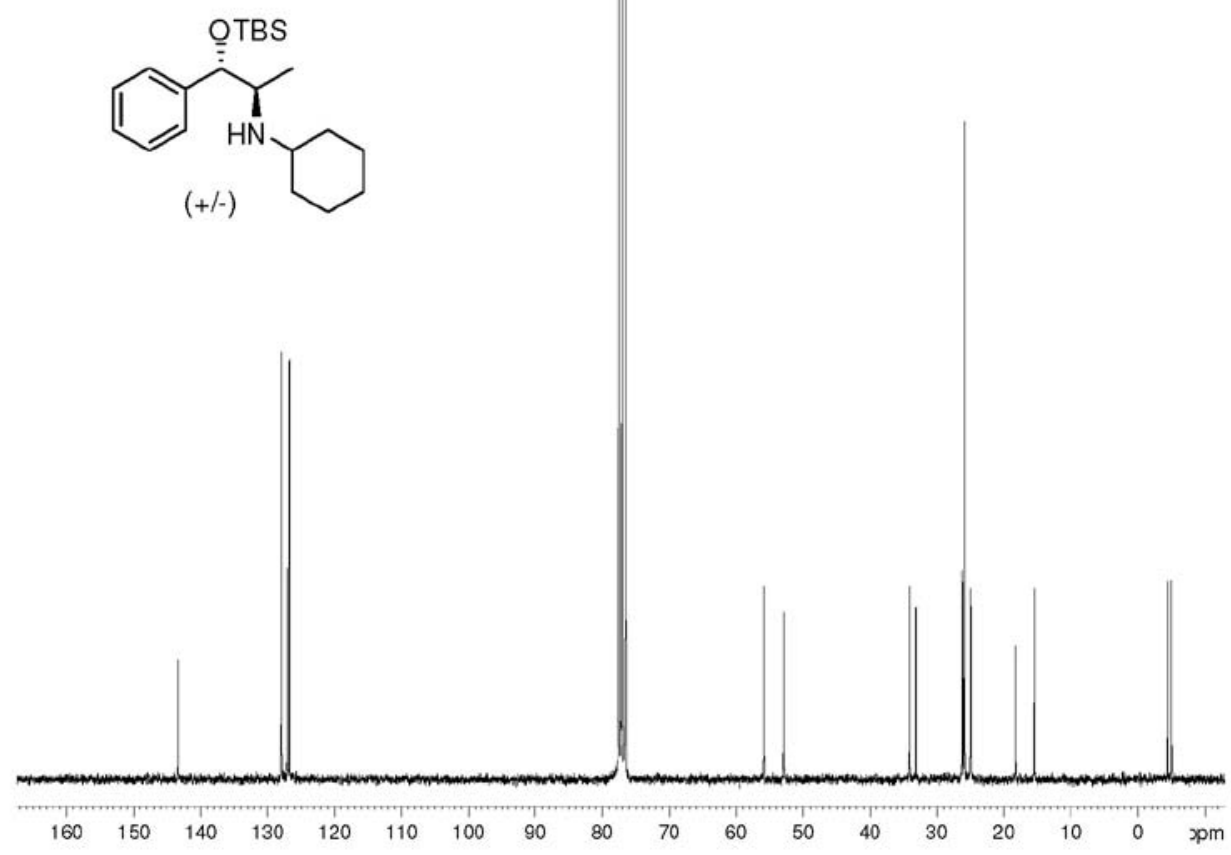

Figure S96. ${ }^{13} \mathrm{C} \mathrm{NMR}\left(\mathrm{CDCl}_{3}, 62.5 \mathrm{MHz}\right)$ of vicinal aminoalcohol 48 .

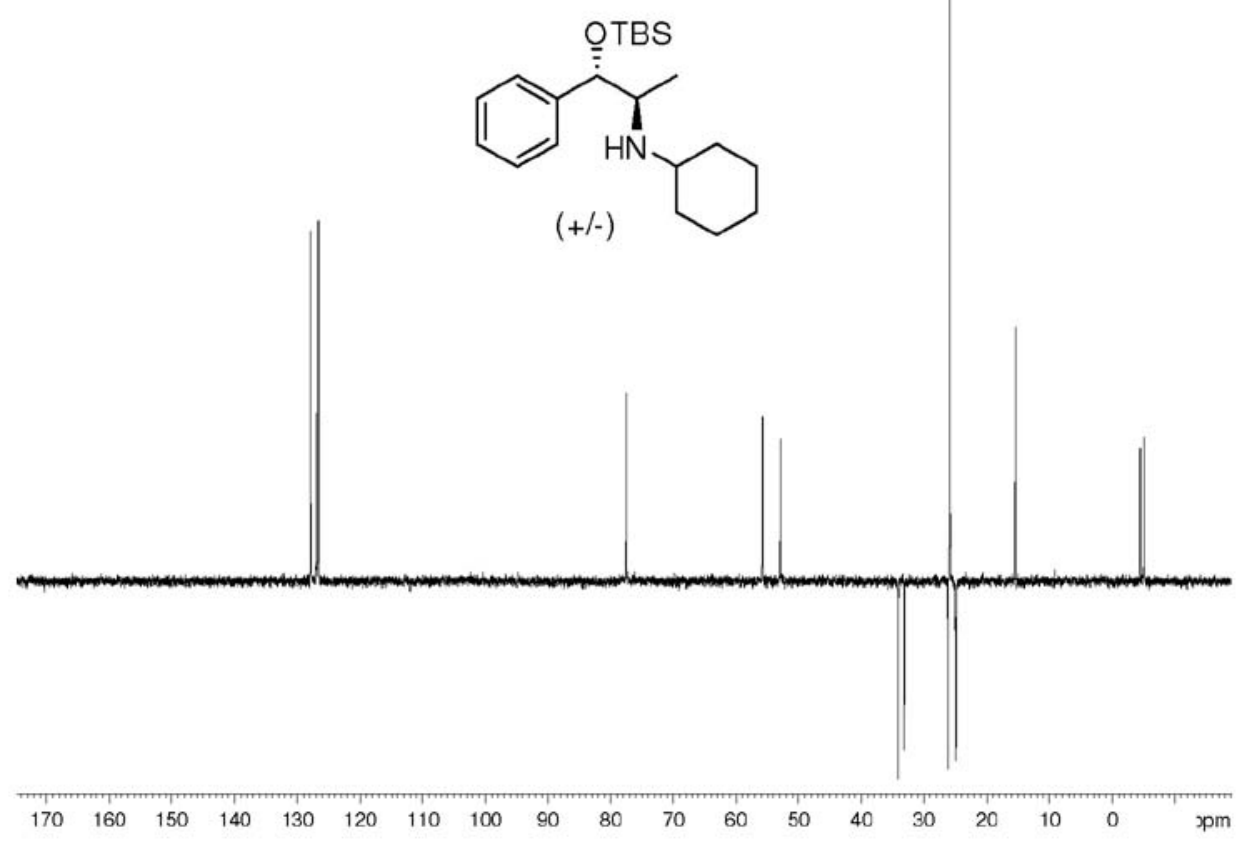

Figure S97. DEPT $135\left(\mathrm{CDCl}_{3}, 62.5 \mathrm{MHz}\right)$ of vicinal aminoalcohol 48. 


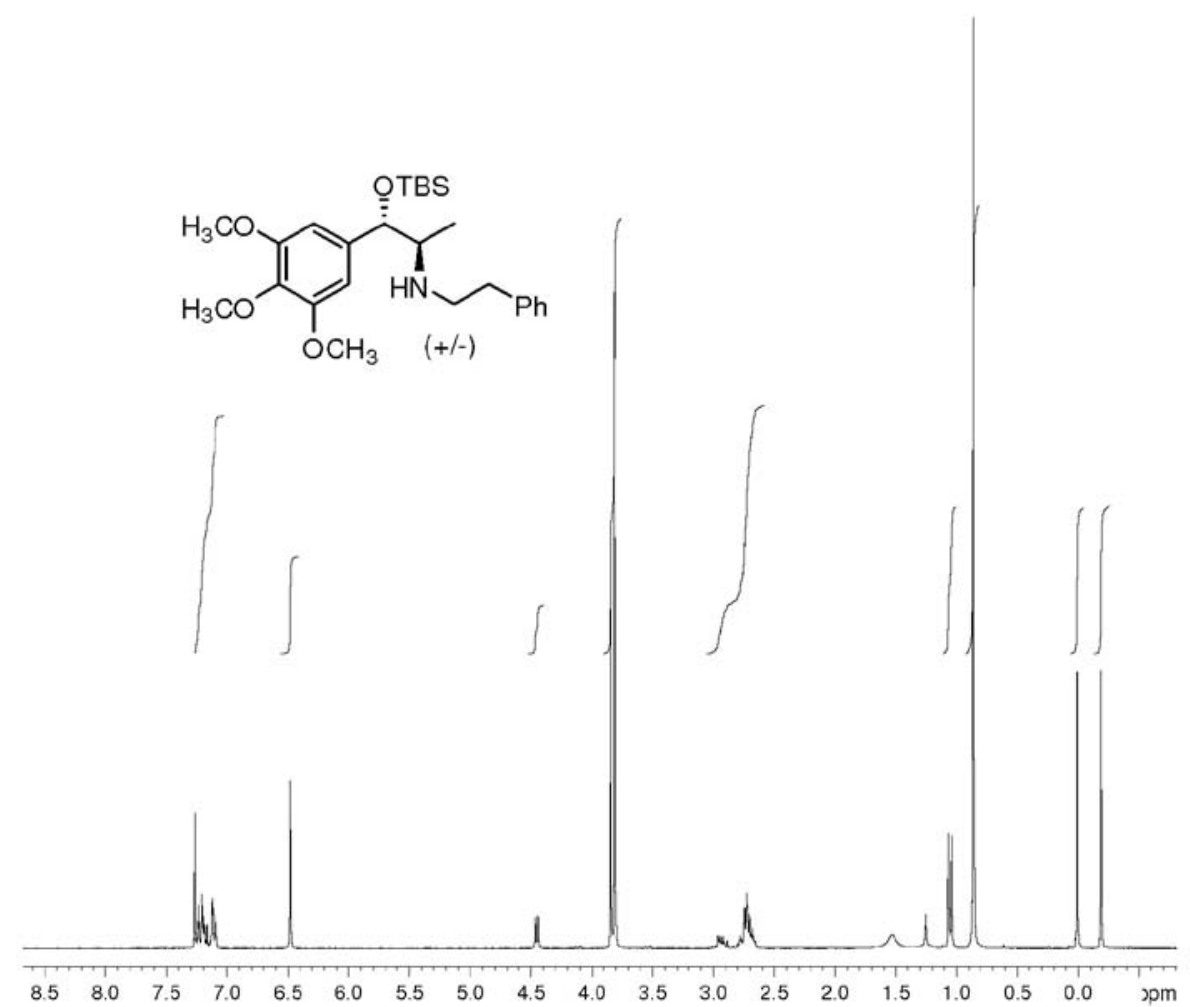

Figure S98. ${ }^{1} \mathrm{H}$ NMR $\left(\mathrm{CDCl}_{3}, 250 \mathrm{MHz}\right)$ of vicinal aminoalchol 49.
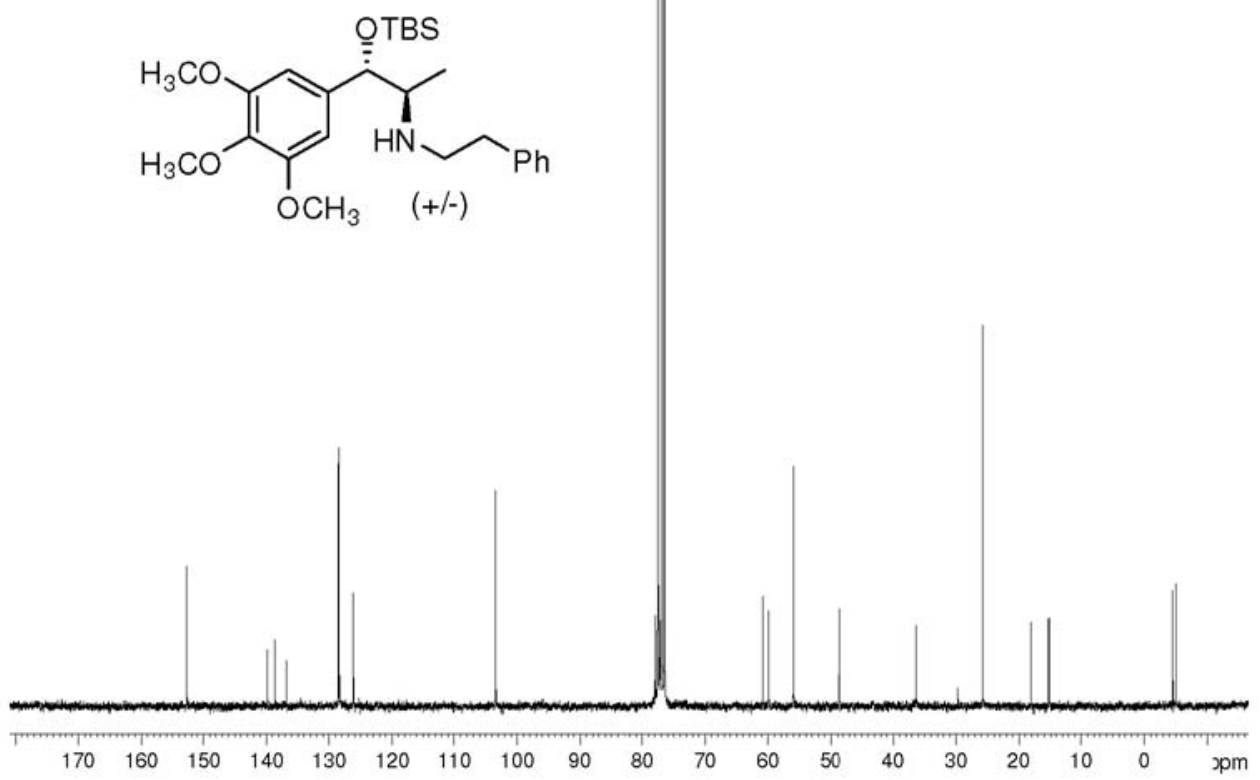

Figure S99. ${ }^{13} \mathrm{C} \mathrm{NMR}\left(\mathrm{CDCl}_{3}, 62.5 \mathrm{MHz}\right)$ of vicinal aminoalcohol 49. 


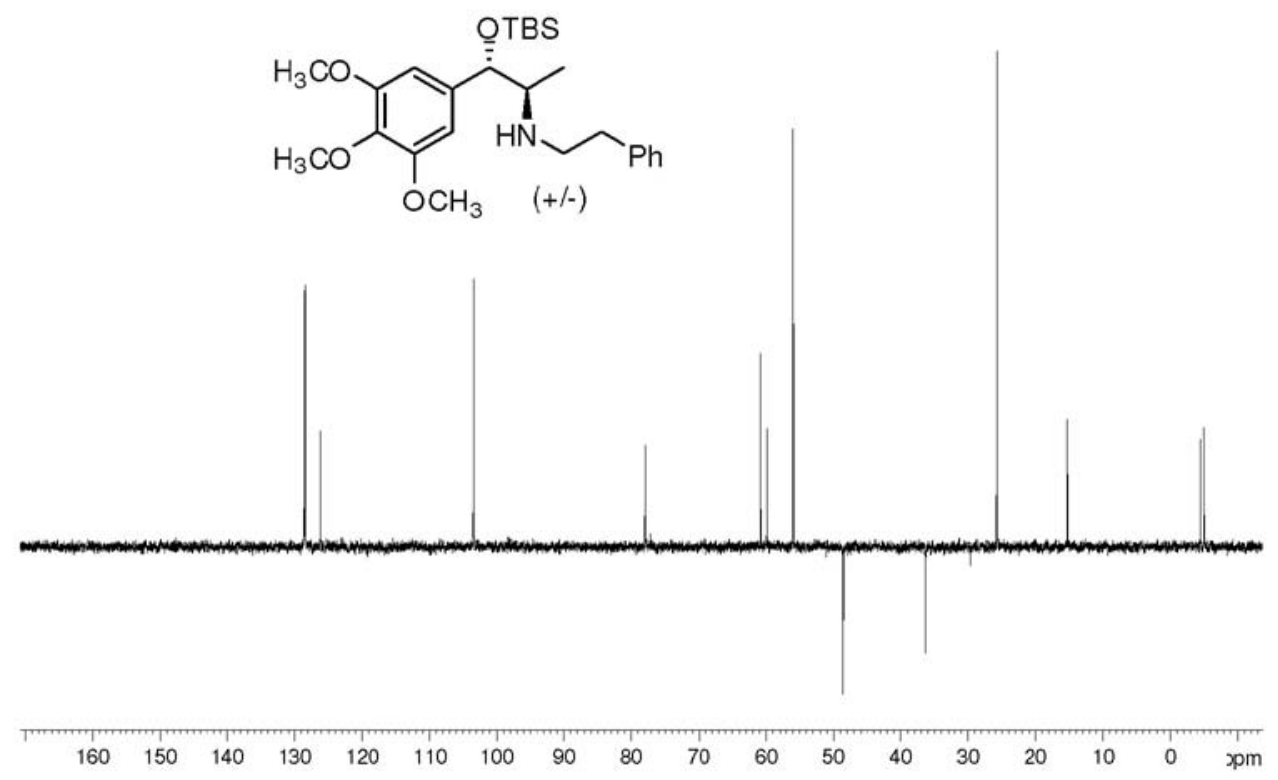

Figure S100. DEPT $135\left(\mathrm{CDCl}_{3}, 62.5 \mathrm{MHz}\right)$ of vicinal aminoalcohol 49.

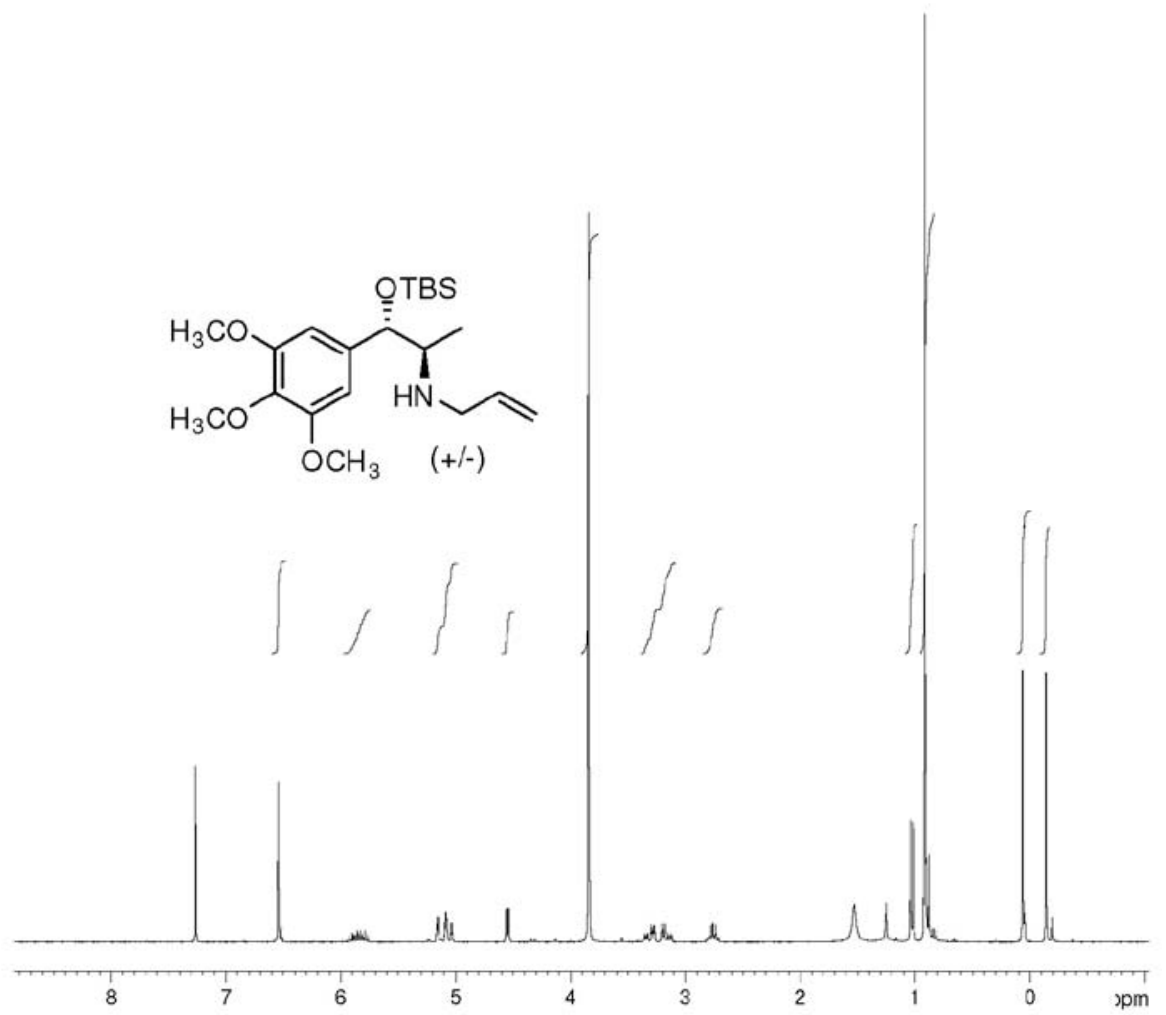

Figure S101. ${ }^{1} \mathrm{H}$ NMR $\left(\mathrm{CDCl}_{3}, 250 \mathrm{MHz}\right)$ of vicinal aminoalcohol $\mathbf{5 0}$. 

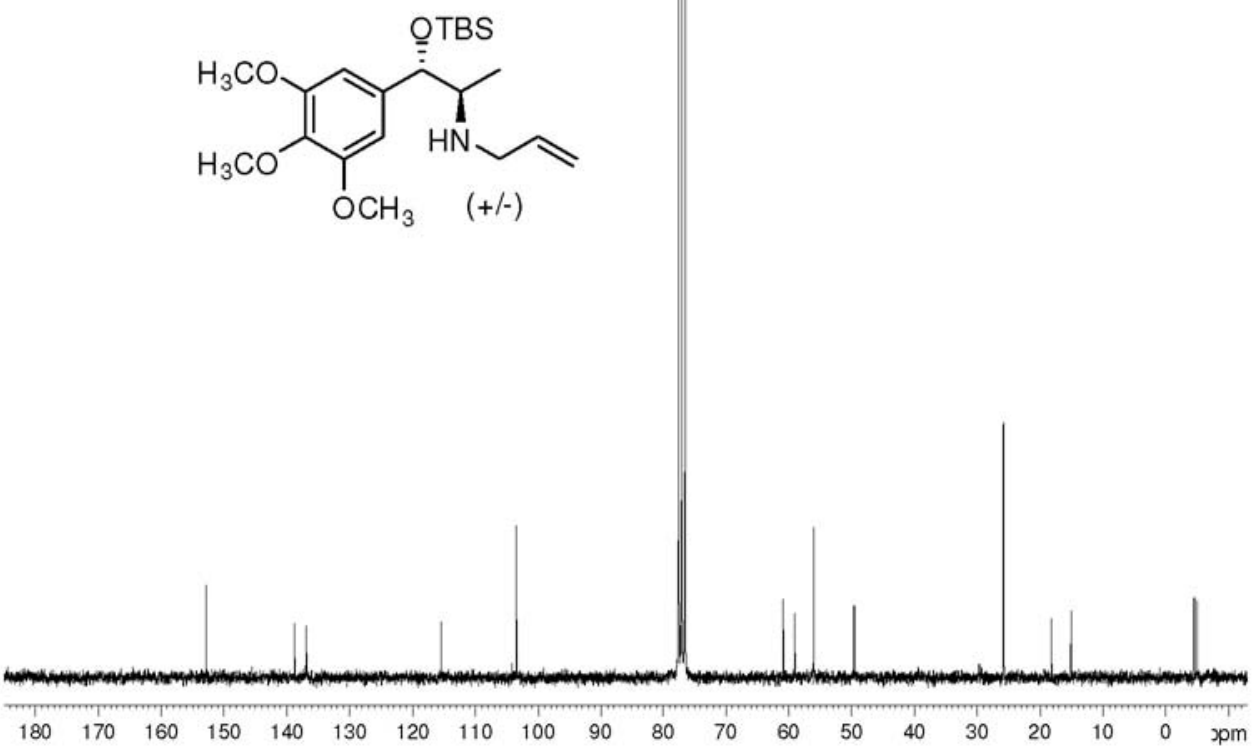

Figure S102. ${ }^{13} \mathrm{C}$ NMR $\left(\mathrm{CDCl}_{3}, 62.5 \mathrm{MHz}\right)$ of vicinal aminoalcohol $\mathbf{5 0}$.
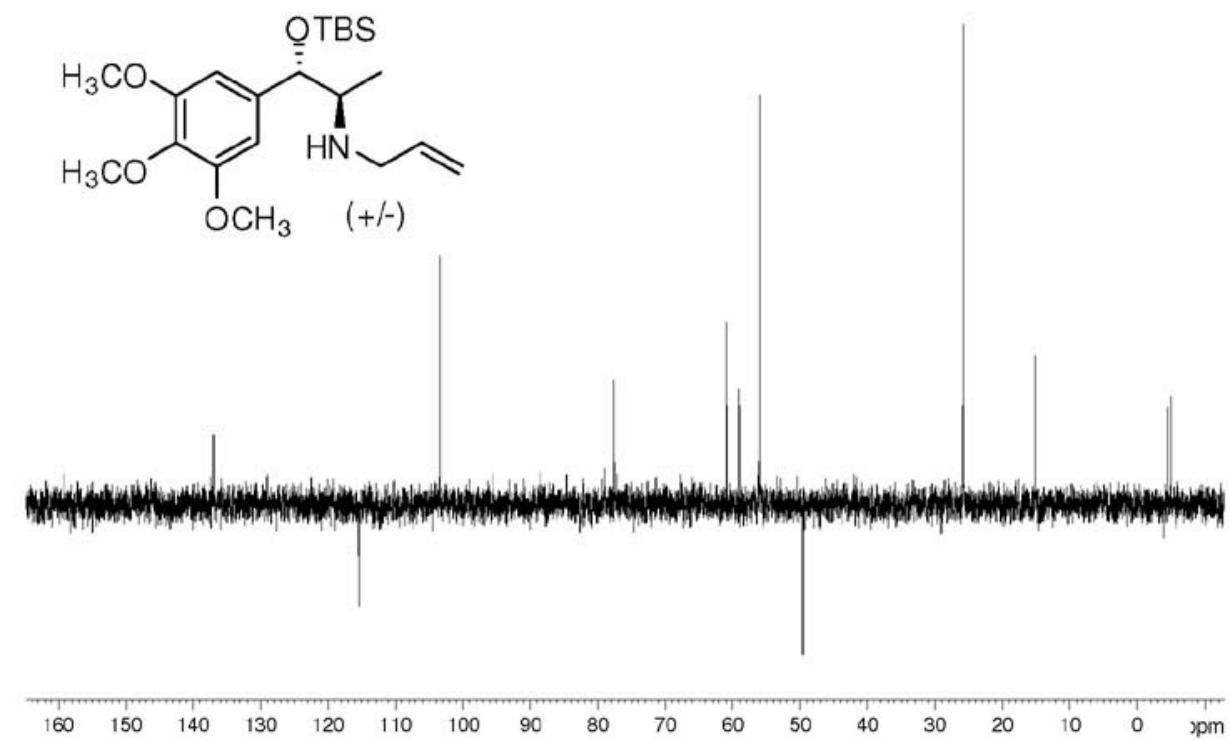

Figure S103. DEPT $135\left(\mathrm{CDCl}_{3}, 62.5 \mathrm{MHz}\right)$ of vicina aminoalcohol $\mathbf{5 0 .}$ 


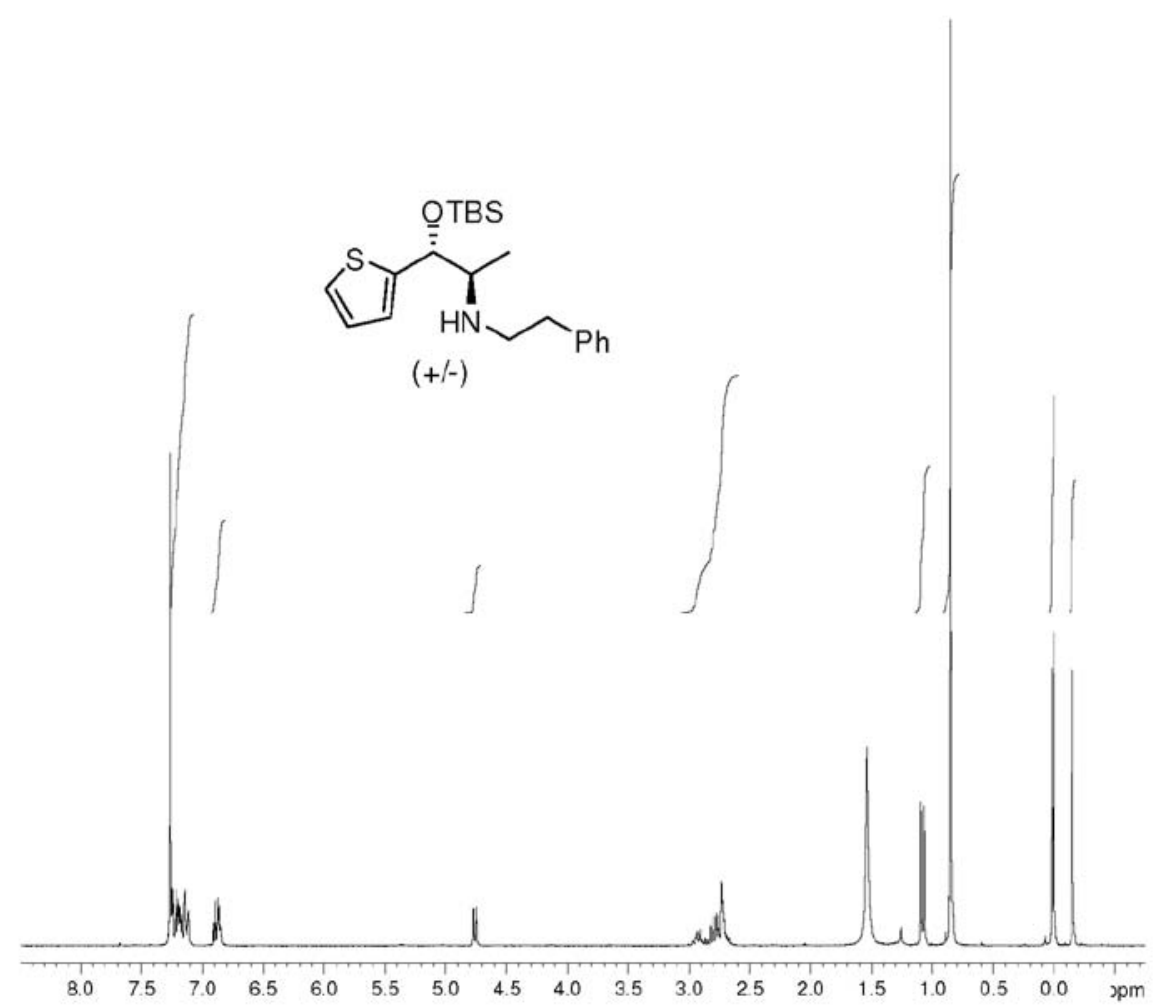

Figure S104. ${ }^{1} \mathrm{H}$ NMR $\left(\mathrm{CDCl}_{3}, 250 \mathrm{MHz}\right)$ of vicinal aminoalcohol 51.

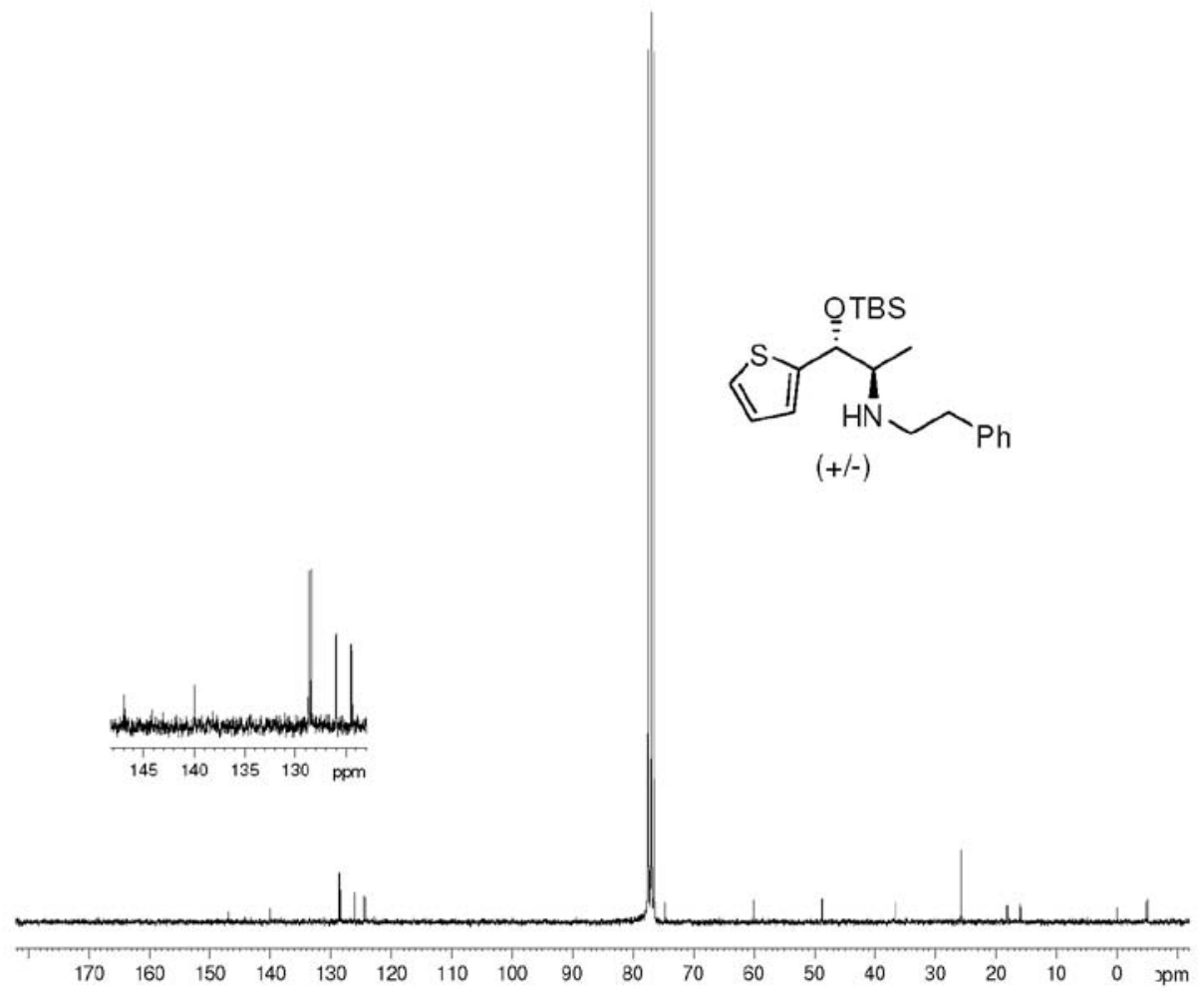

Figure S105. ${ }^{13} \mathrm{C}$ NMR $\left(\mathrm{CDCl}_{3}, 62.5 \mathrm{MHz}\right)$ of vicinal aminoalcohol 51. 


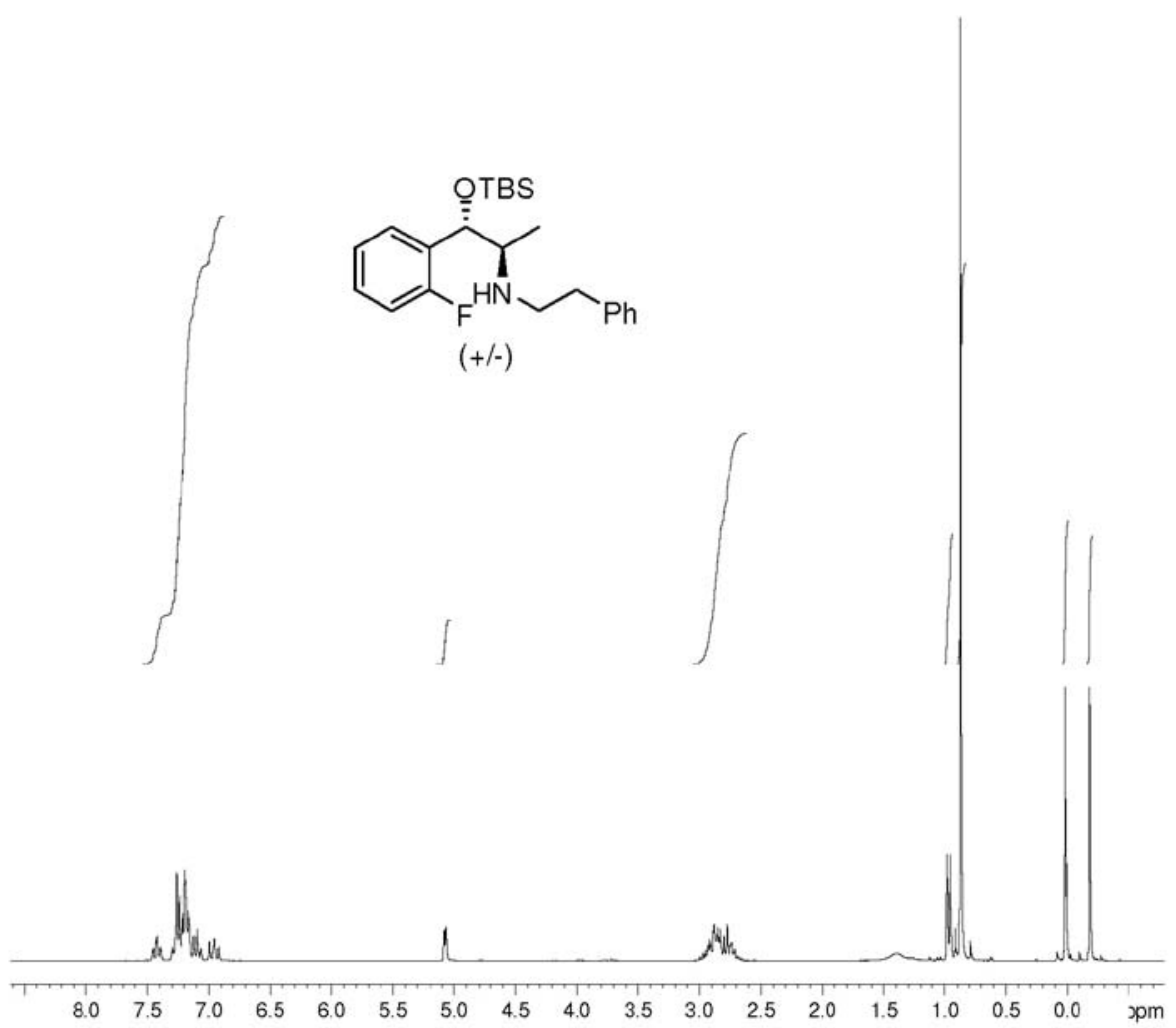

Figure S106. ${ }^{1} \mathrm{H} \mathrm{NMR}\left(\mathrm{CDCl}_{3}, 250 \mathrm{MHz}\right)$ of vicinal aminoalcohol 52.

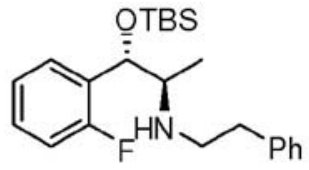

$(+/-)$

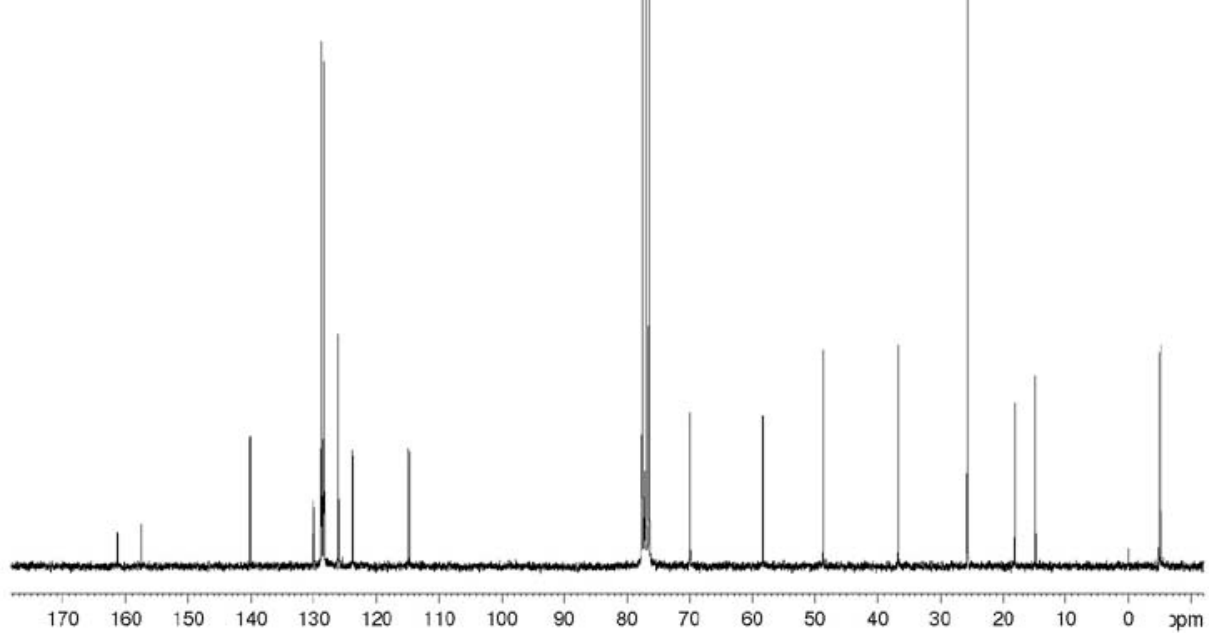

Figure S107. ${ }^{13} \mathrm{C}$ NMR $\left(\mathrm{CDCl}_{3}, 62.5 \mathrm{MHz}\right)$ of vicinal aminoalcohol 52. 


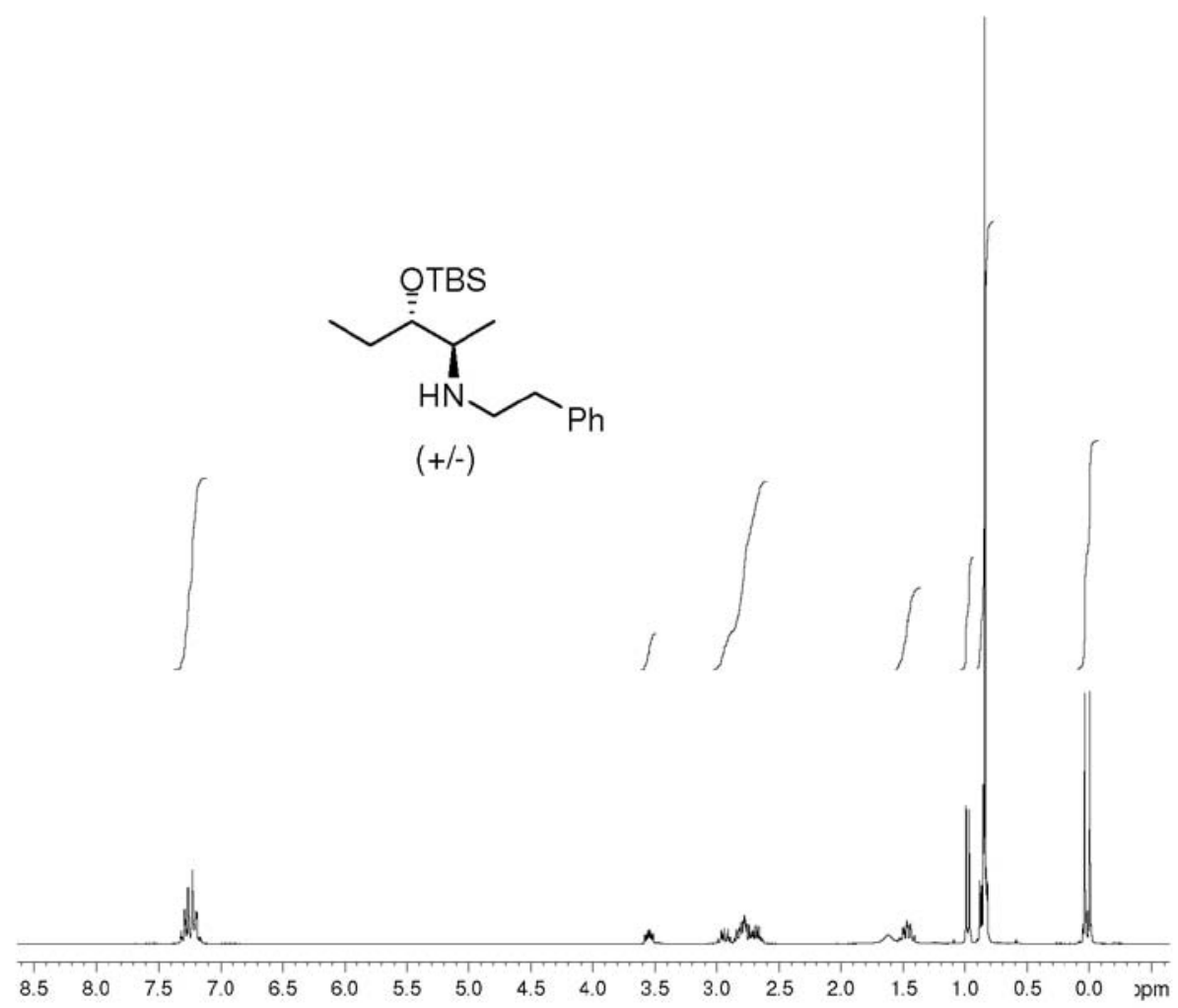

Figure S108. ${ }^{1} \mathrm{H}$ NMR $\left(\mathrm{CDCl}_{3}, 250 \mathrm{MHz}\right)$ of vicinal aminoalcohol 53.

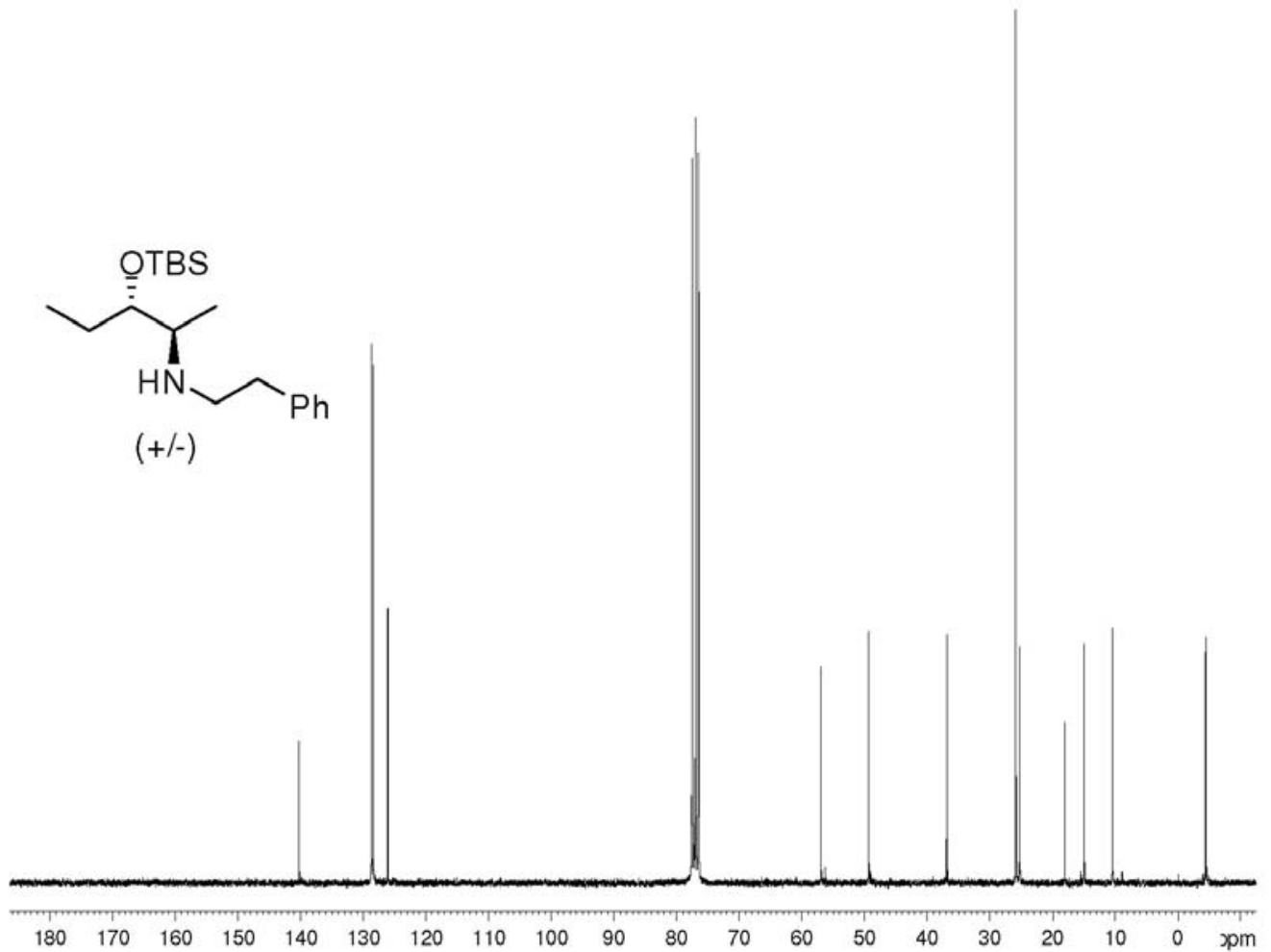

Figure S109. ${ }^{13} \mathrm{C}$ NMR $\left(\mathrm{CDCl}_{3}, 62.5 \mathrm{MHz}\right)$ of vicinal aminoalcohol 53. 


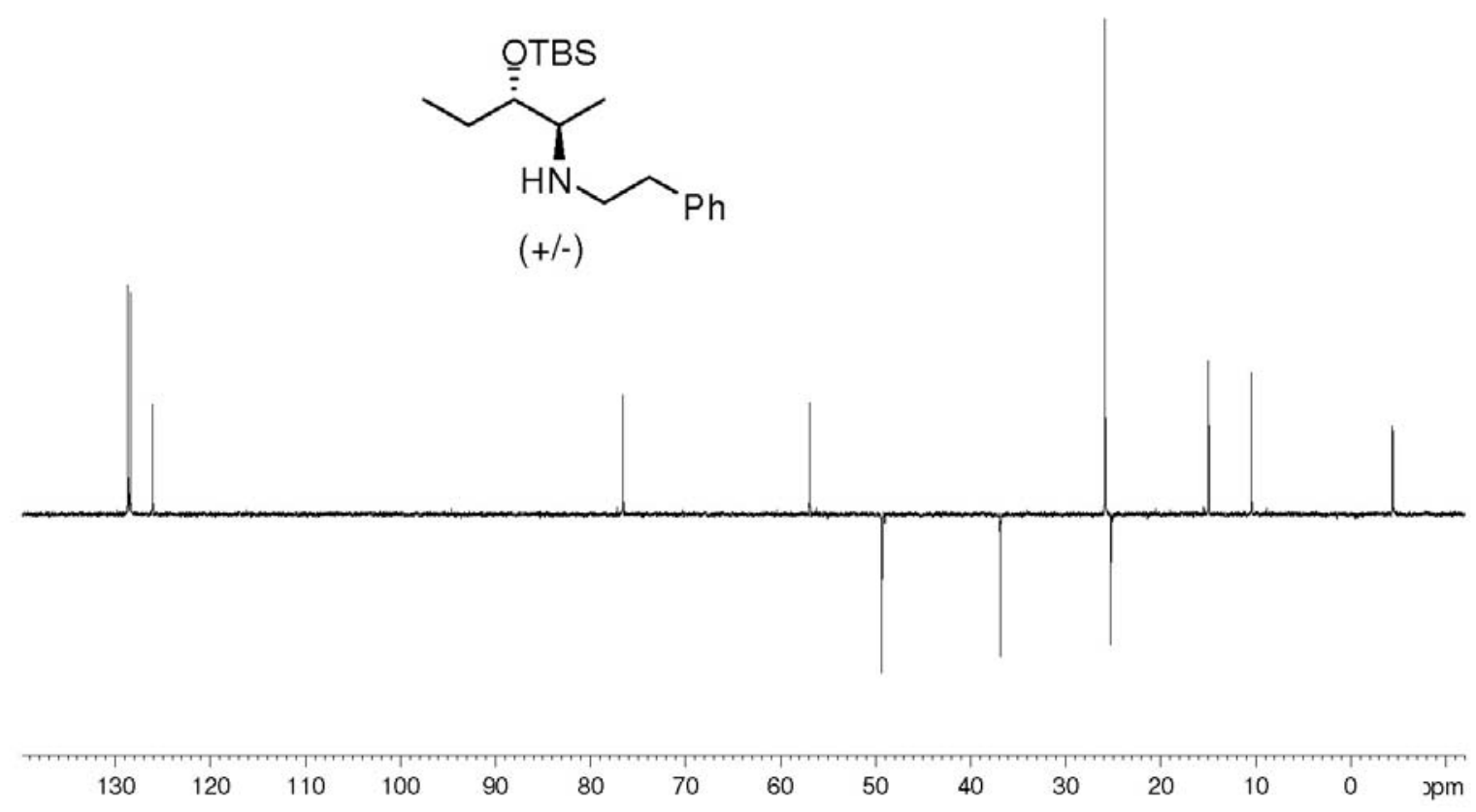

Figure S110. DEPT $135\left(\mathrm{CDCl}_{3}, 62.5 \mathrm{MHz}\right)$ of vicinal aminoalcohol 53.

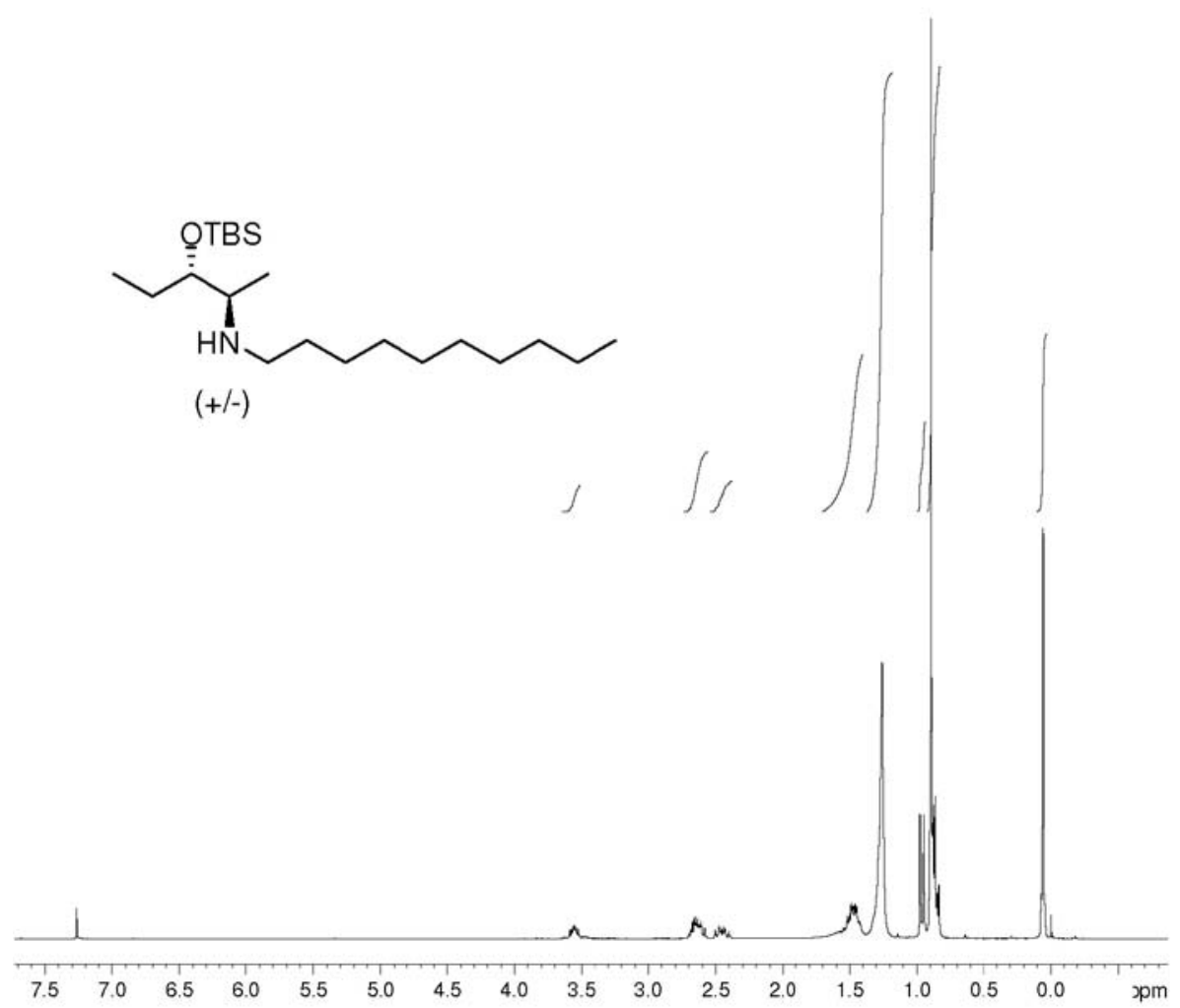

Figure S111. ${ }^{1} \mathrm{H}$ NMR $\left(\mathrm{CDCl}_{3}, 250 \mathrm{MHz}\right)$ of vicinal aminoalcohol 54. 


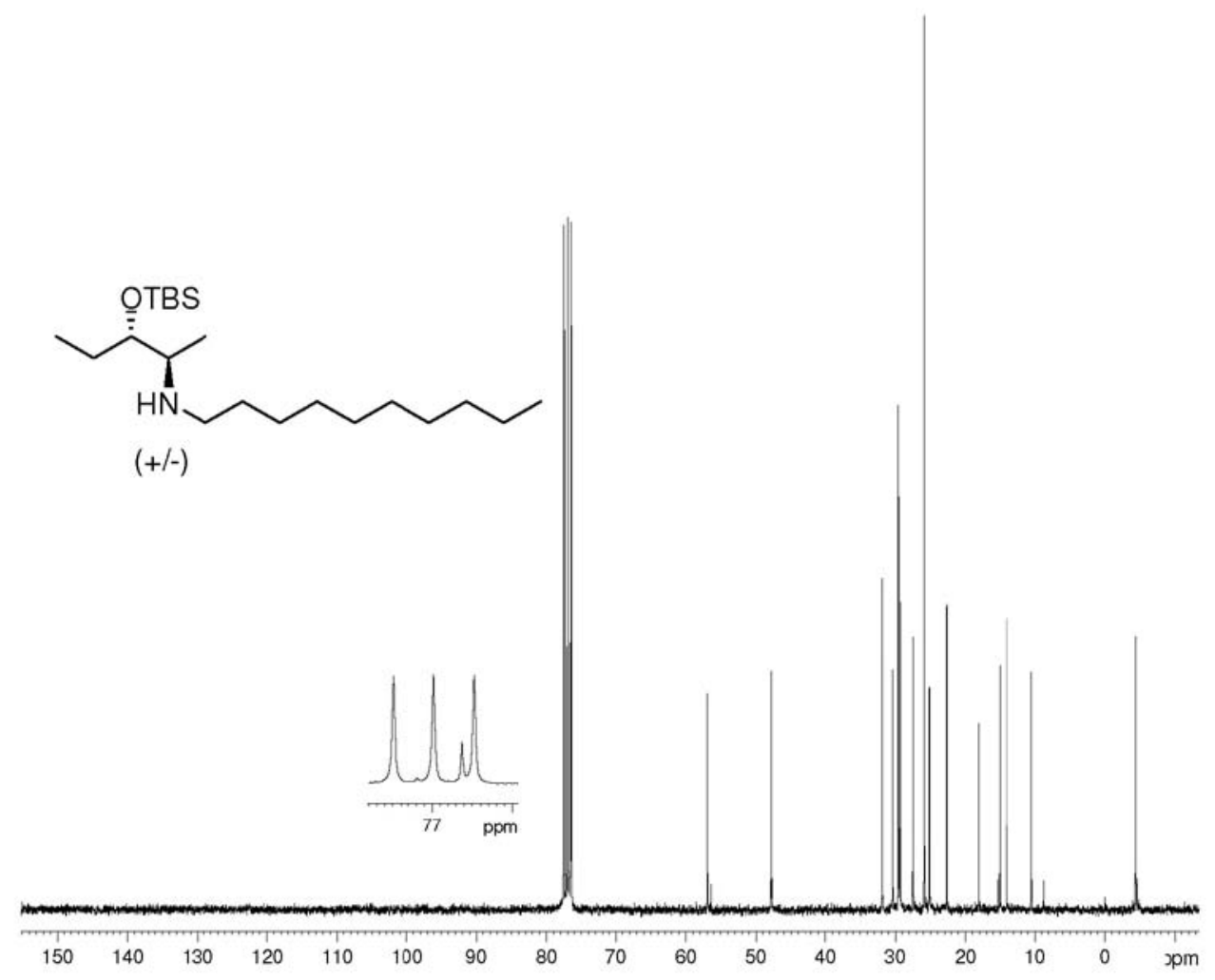

Figure S112. ${ }^{13} \mathrm{C} \mathrm{NMR}\left(\mathrm{CDCl}_{3}, 62.5 \mathrm{MHz}\right)$ of vicinal aminoalcohol 54.
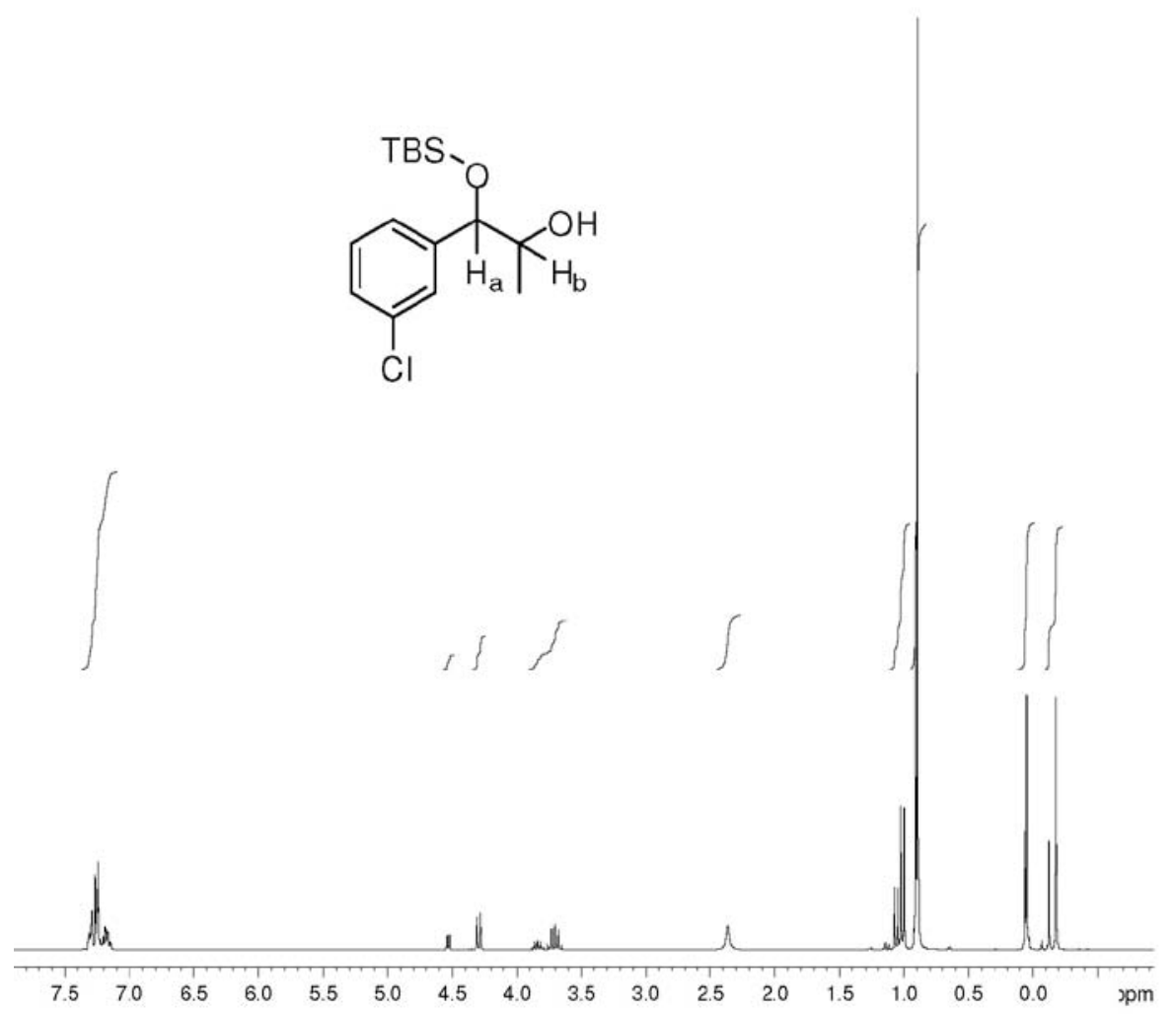

Figure S113. ${ }^{1} \mathrm{H}$ NMR $\left(\mathrm{CDCl}_{3}, 250 \mathrm{MHz}\right)$ of monosilylated diol 60 . 
<smiles>CC(C)(C)C(O[As])(c1cccc(Cl)c1)C(C)(C)O</smiles>

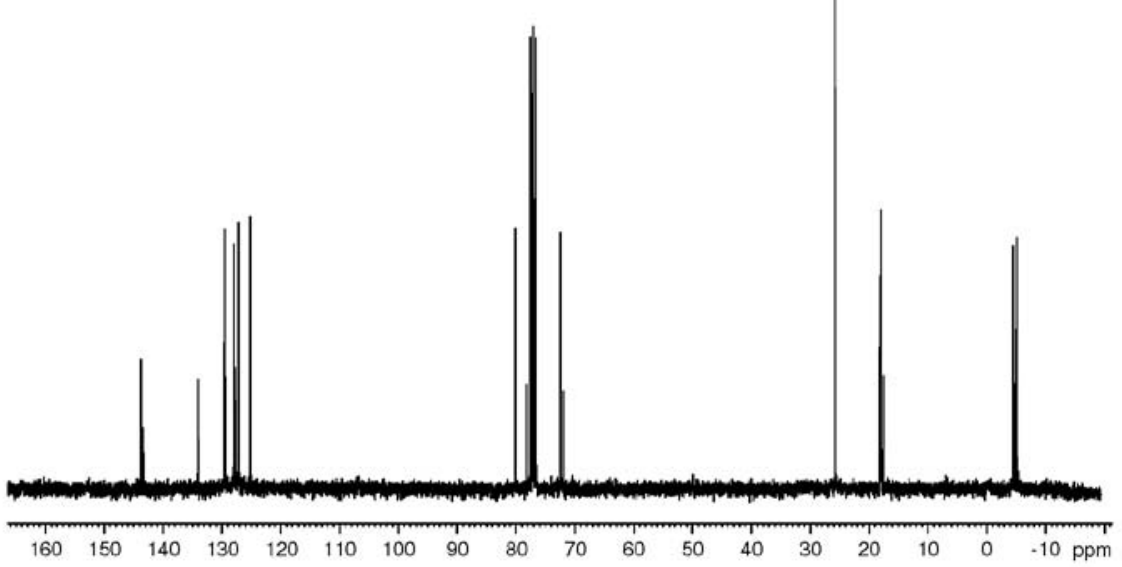

Figure S114. ${ }^{13} \mathrm{C}$ NMR $\left(\mathrm{CDCl}_{3}, 75.4 \mathrm{MHz}\right)$ of monosilylated diol 60 .

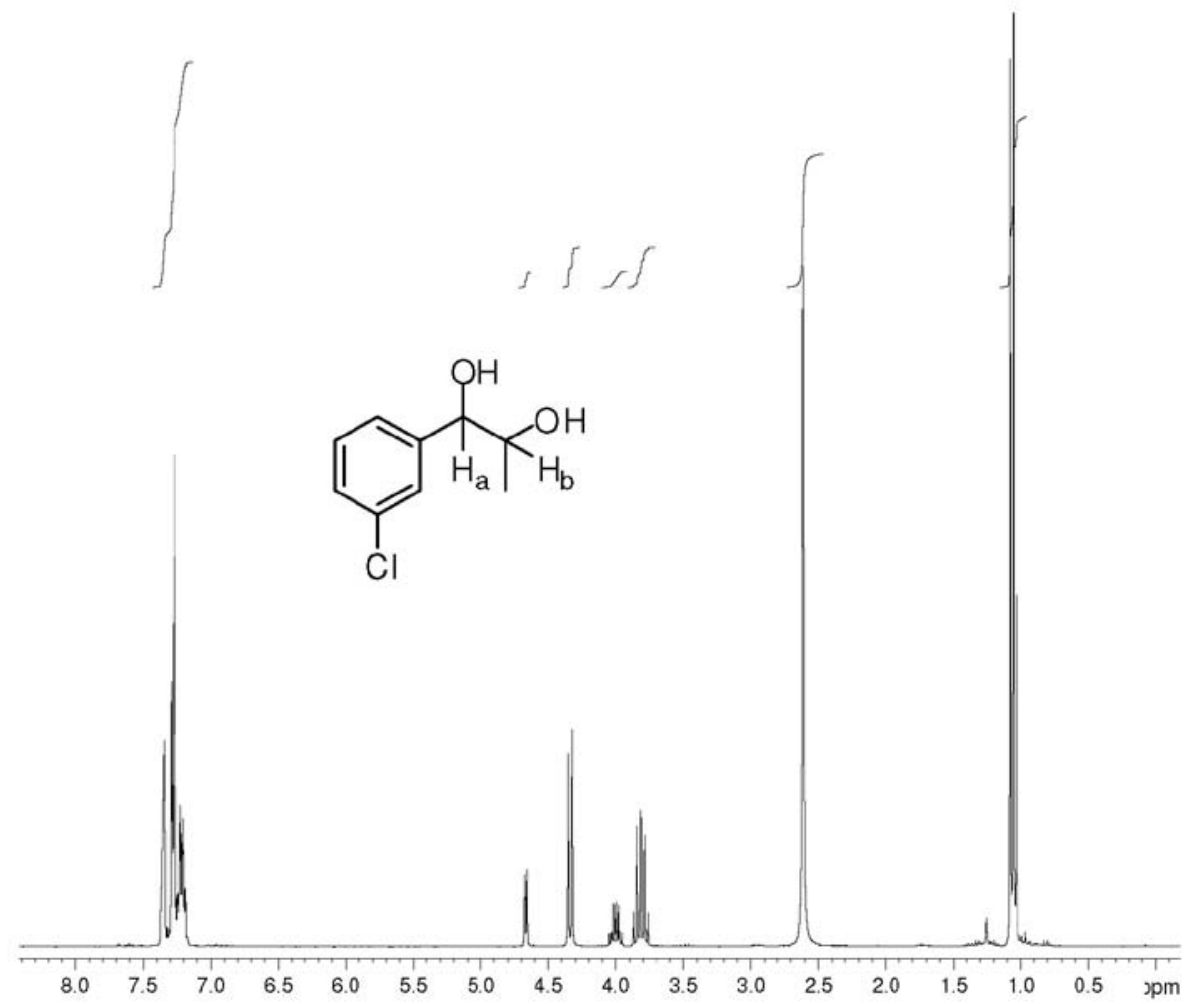

Figure S115. ${ }^{1} \mathrm{H}$ NMR $\left(\mathrm{CDCl}_{3}, 250 \mathrm{MHz}\right)$ of diol 61. 


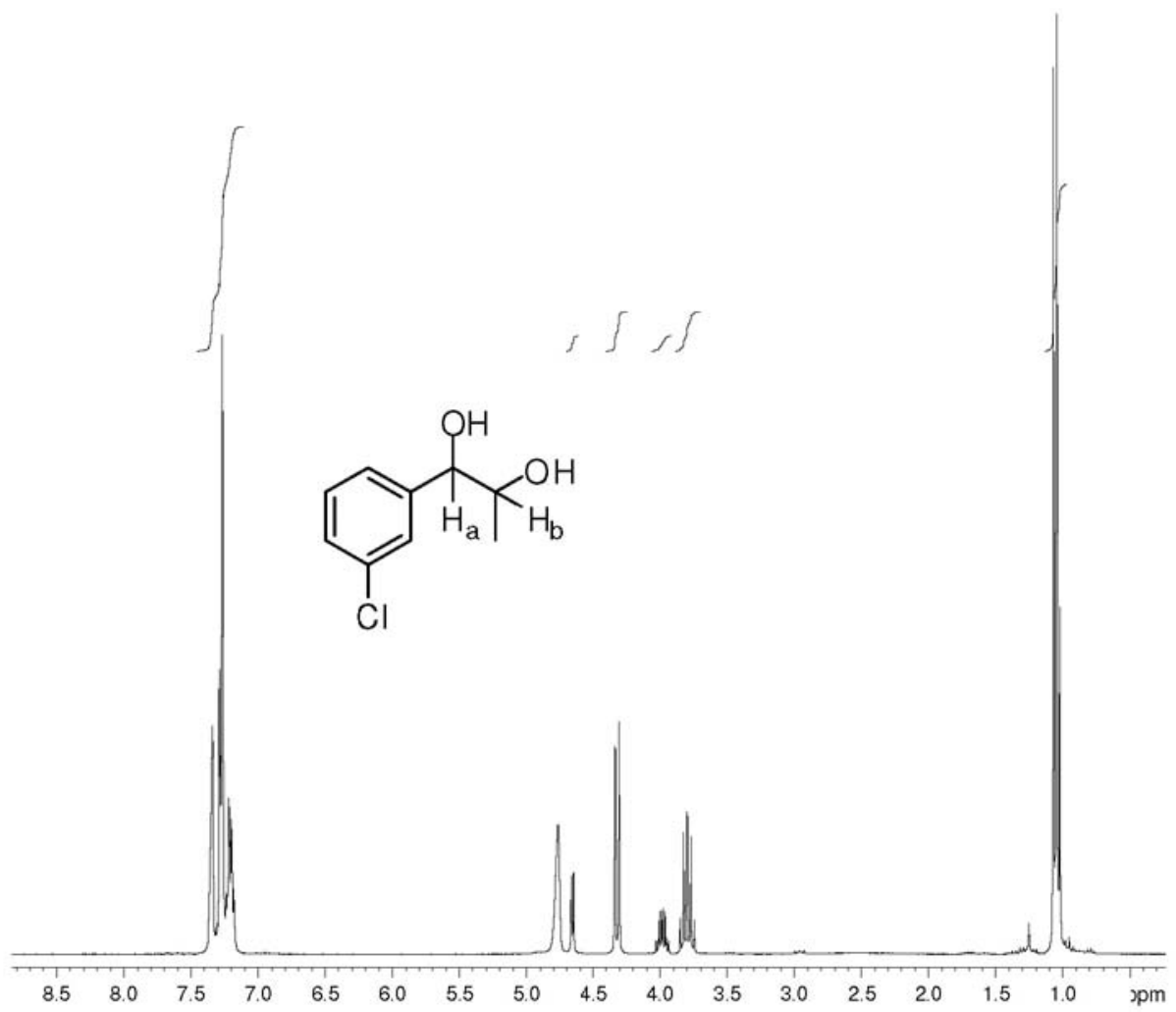

Figure S116. ${ }^{1} \mathrm{H}$ NMR $\left(\mathrm{CDCl}_{3}+2\right.$ drops of $\left.\mathrm{D}_{2} \mathrm{O}, 250 \mathrm{MHz}\right)$ of diol 61 .

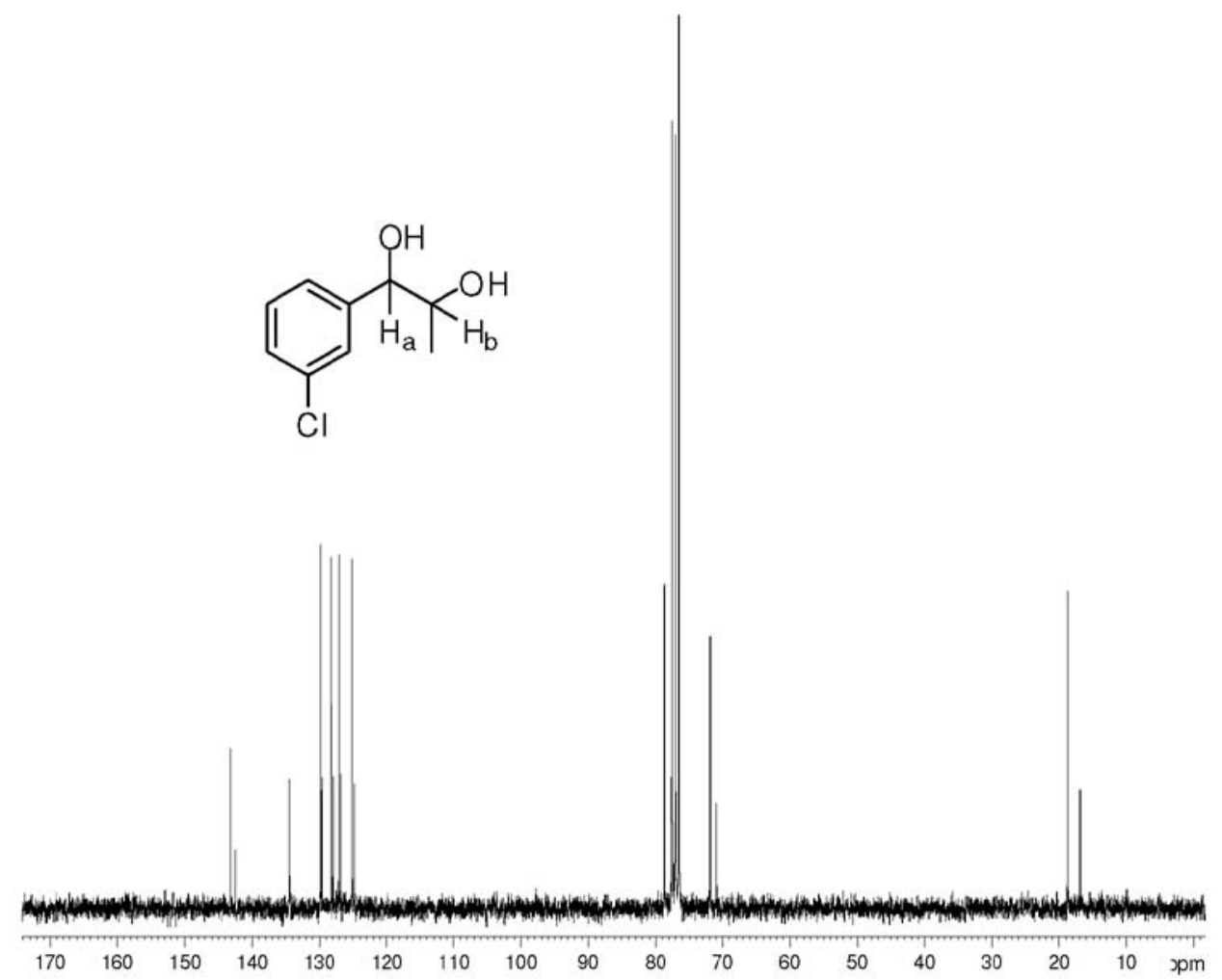

Figure S117. ${ }^{13} \mathrm{C}$ NMR $\left(\mathrm{CDCl}_{3}, 62.5 \mathrm{MHz}\right)$ of diol 61 


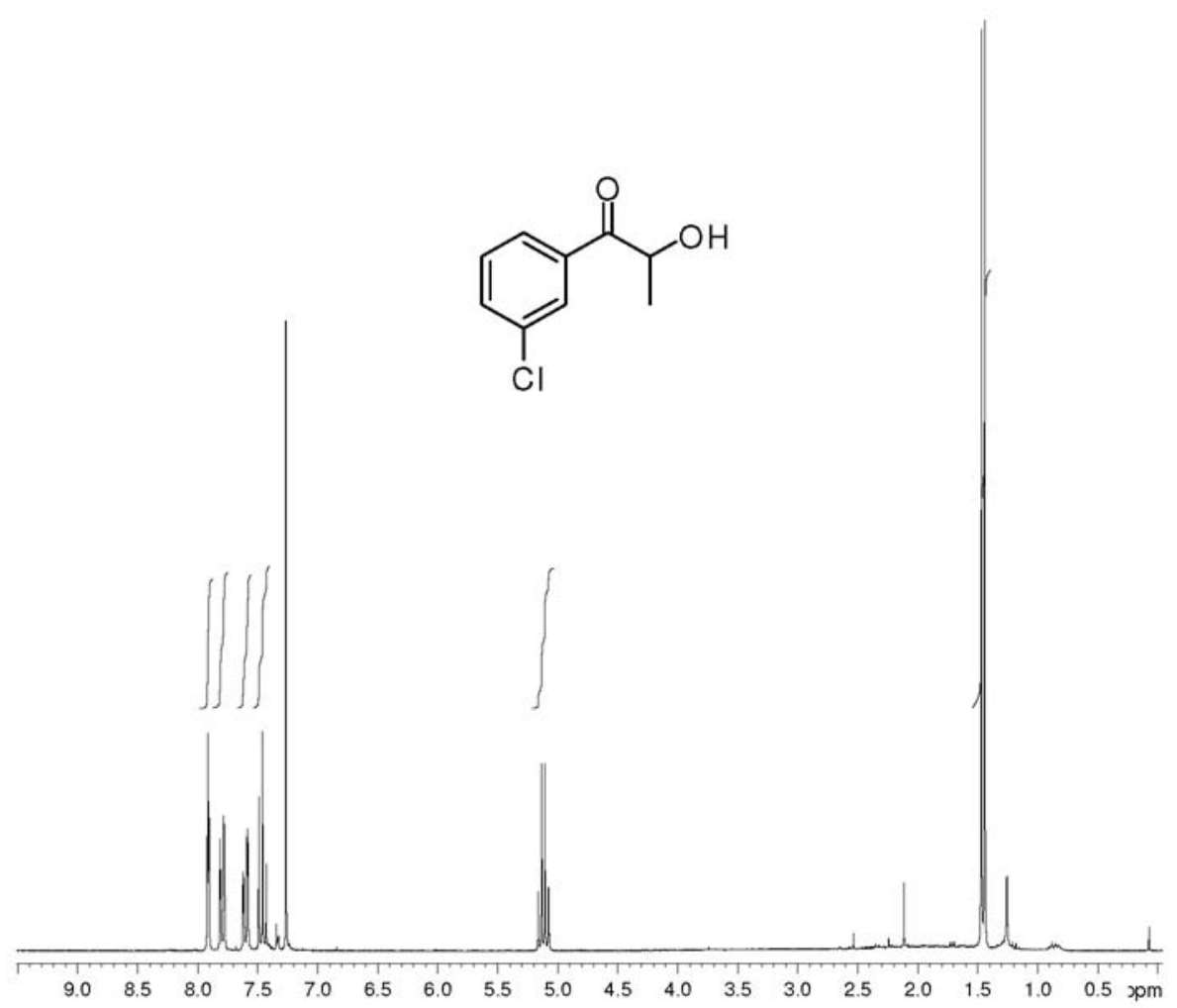

Figure S118. ${ }^{\mathrm{H}} \mathrm{NMR}\left(\mathrm{CDCl}_{3}, 250 \mathrm{MHz}\right)$ of acyloin $\mathbf{5 9}$.

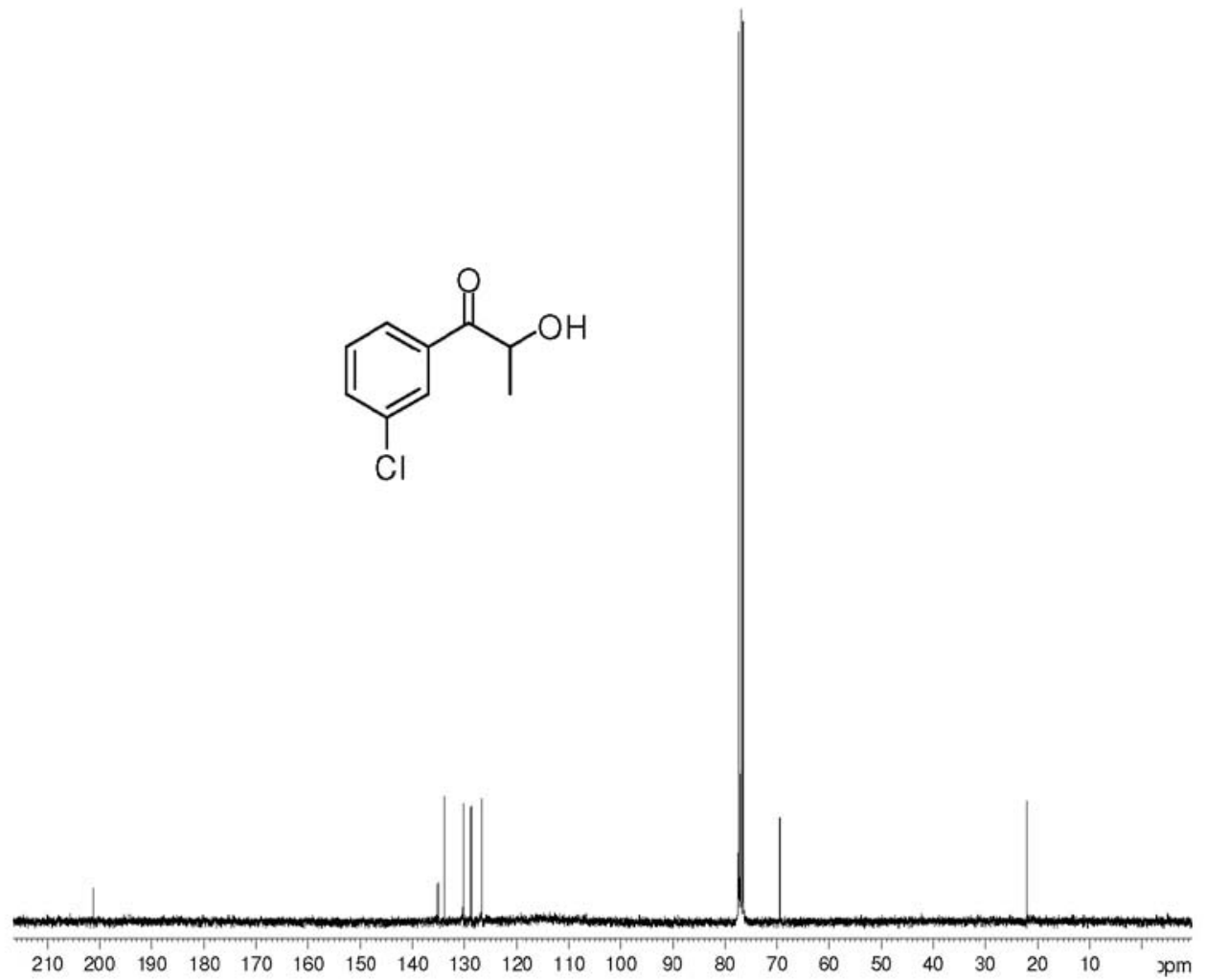

Figure S119. ${ }^{13} \mathrm{C} \mathrm{NMR}\left(\mathrm{CDCl}_{3}, 75.4 \mathrm{MHz}\right)$ of acyloin $\mathbf{5 9}$. 


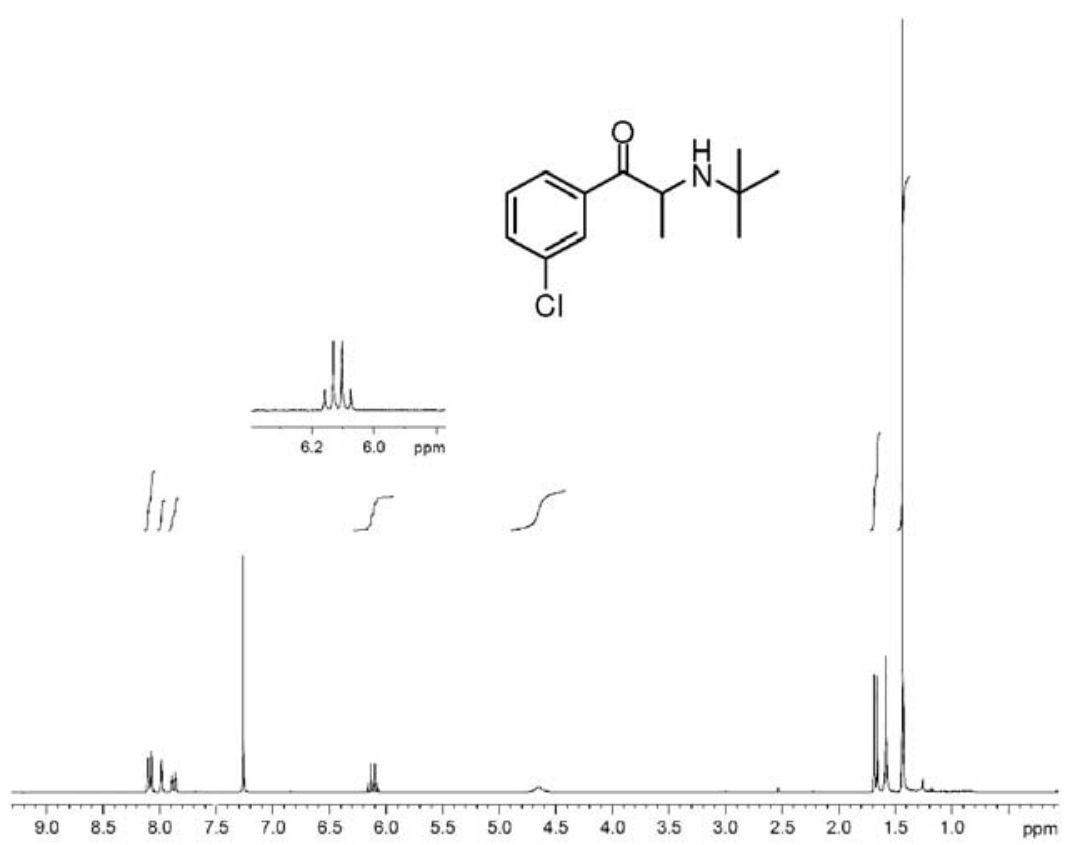

Figure S120. ${ }^{1} \mathrm{H} \mathrm{NMR}\left(\mathrm{CDCl}_{3}, 250 \mathrm{MHz}\right)$ of bupropion (1).

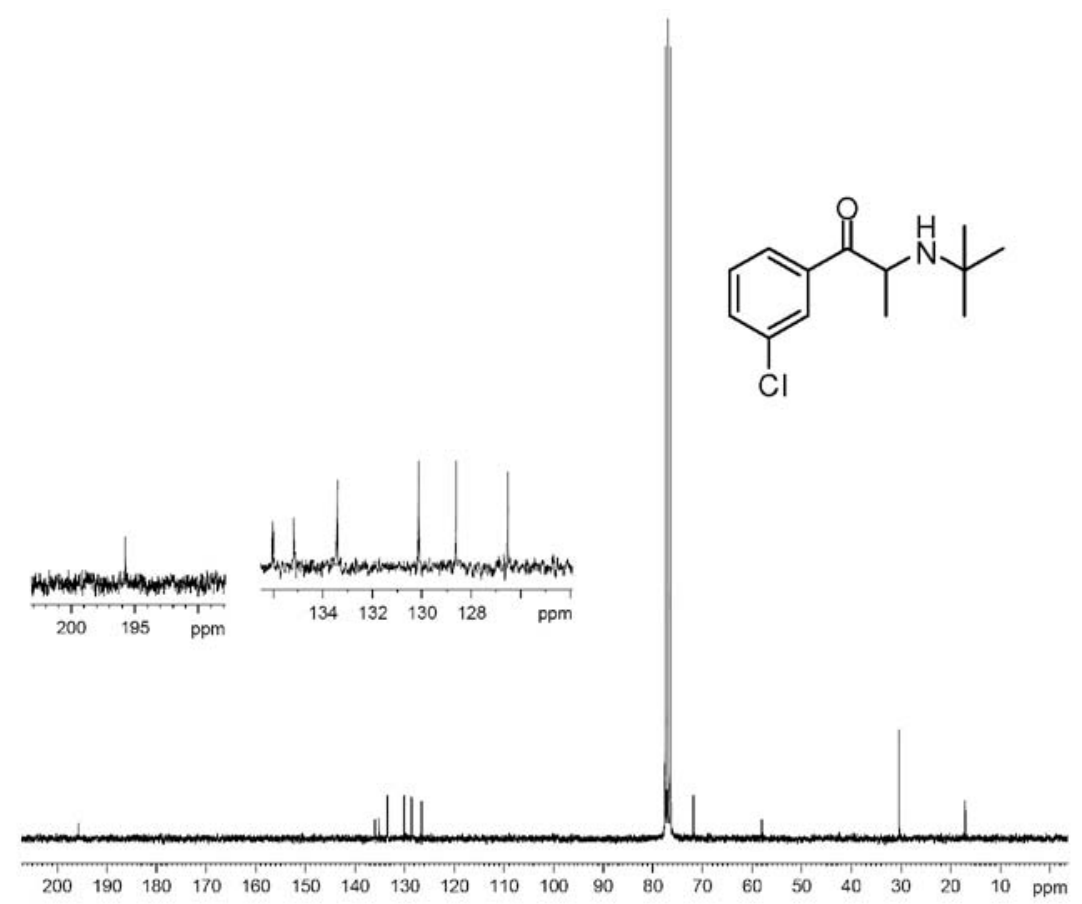

Figure S121. ${ }^{13} \mathrm{C}$ NMR $\left(\mathrm{CDCl}_{3}, 62.5 \mathrm{MHz}\right)$ of bupropion (1). 


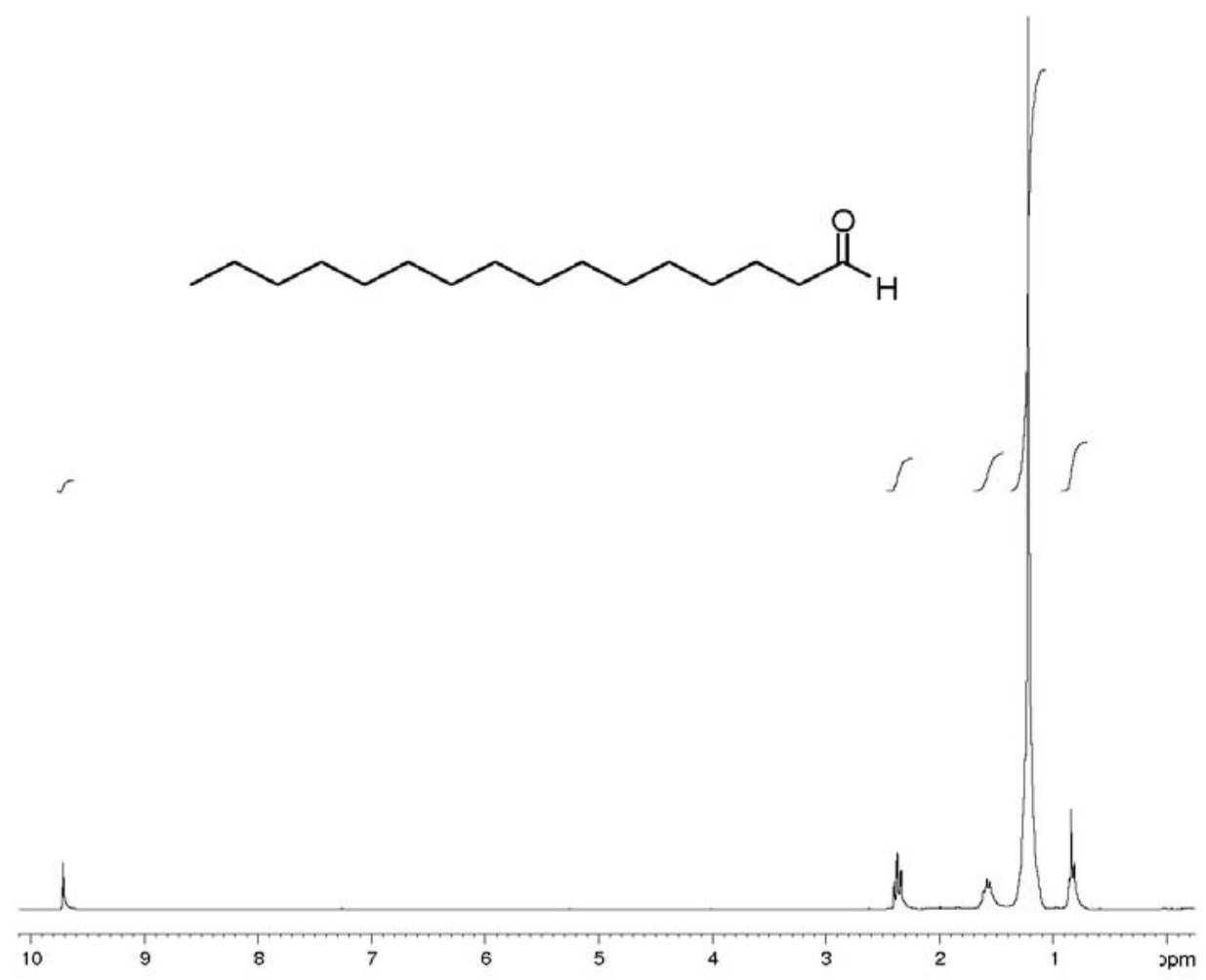

Figure S122. ${ }^{1} \mathrm{H} \mathrm{NMR}\left(\mathrm{CDCl}_{3}, 250 \mathrm{MHz}\right)$ of hexadecanal.

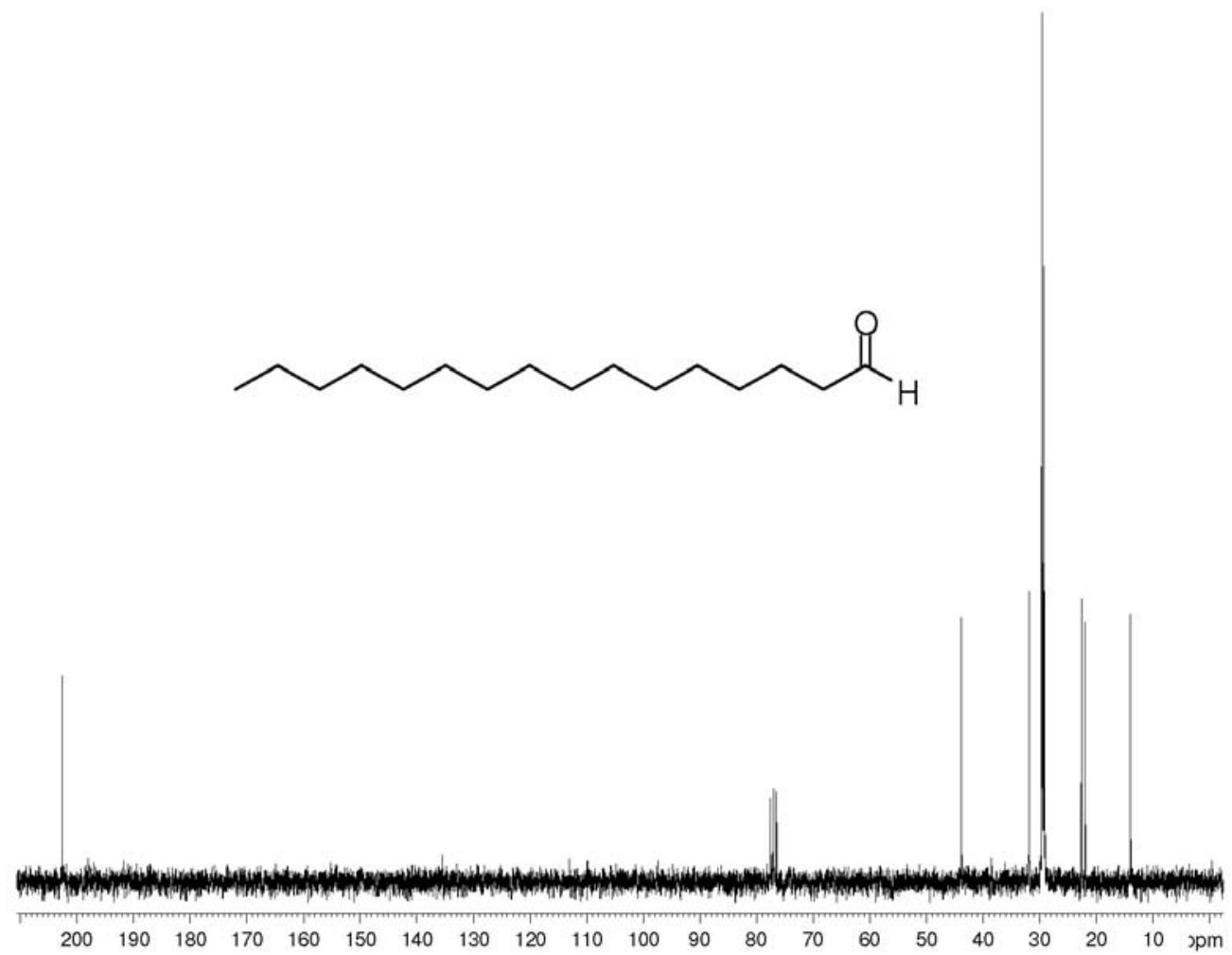

Figure S123. ${ }^{13} \mathrm{C}$ NMR $\left(\mathrm{CDCl}_{3}, 62.5 \mathrm{MHz}\right)$ of hexadecanal. 


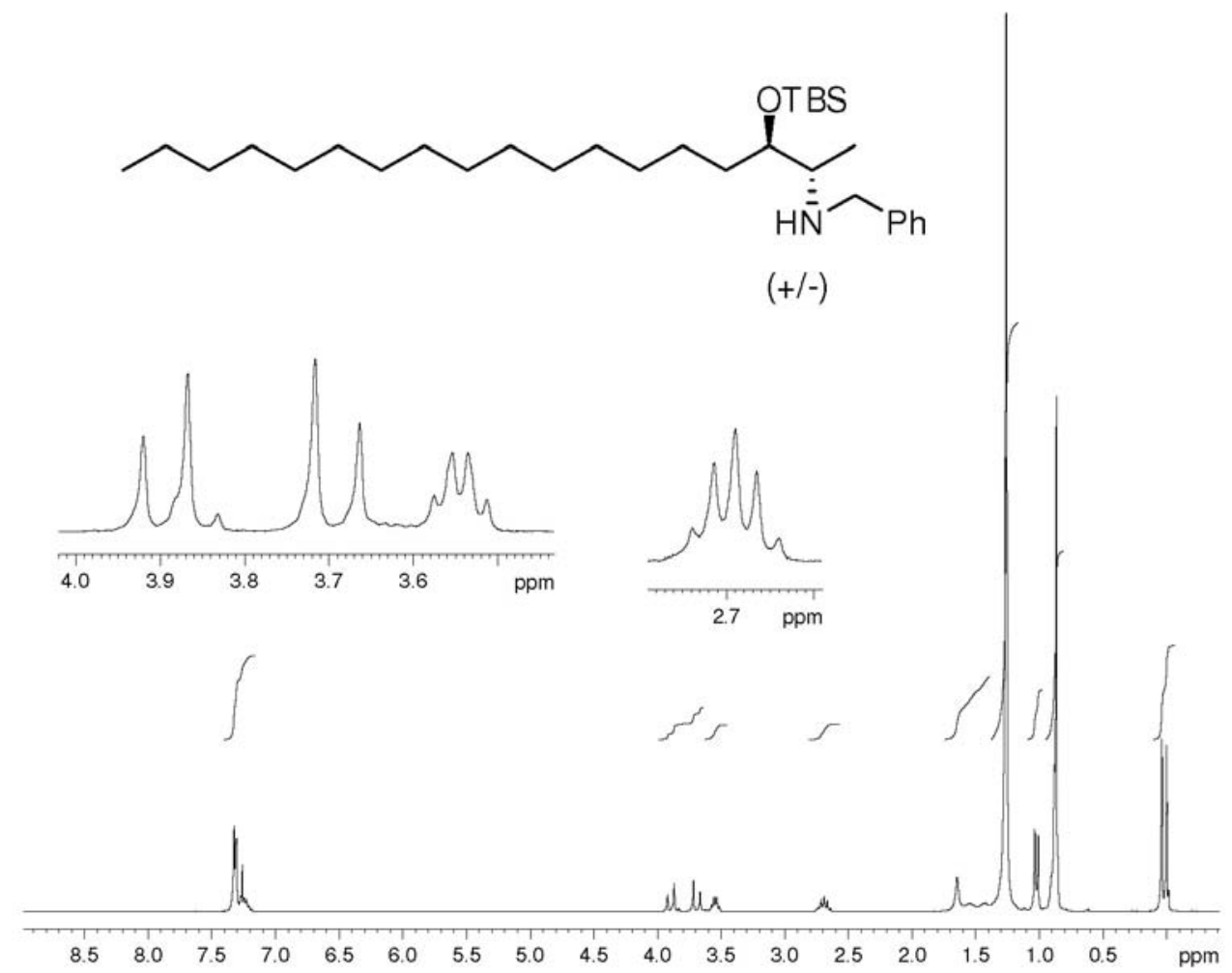

Figure S124. ${ }^{1} \mathrm{H}$ NMR $\left(\mathrm{CDCl}_{3}, 250 \mathrm{MHz}\right)$ of vicinal aminoalcohol 62.

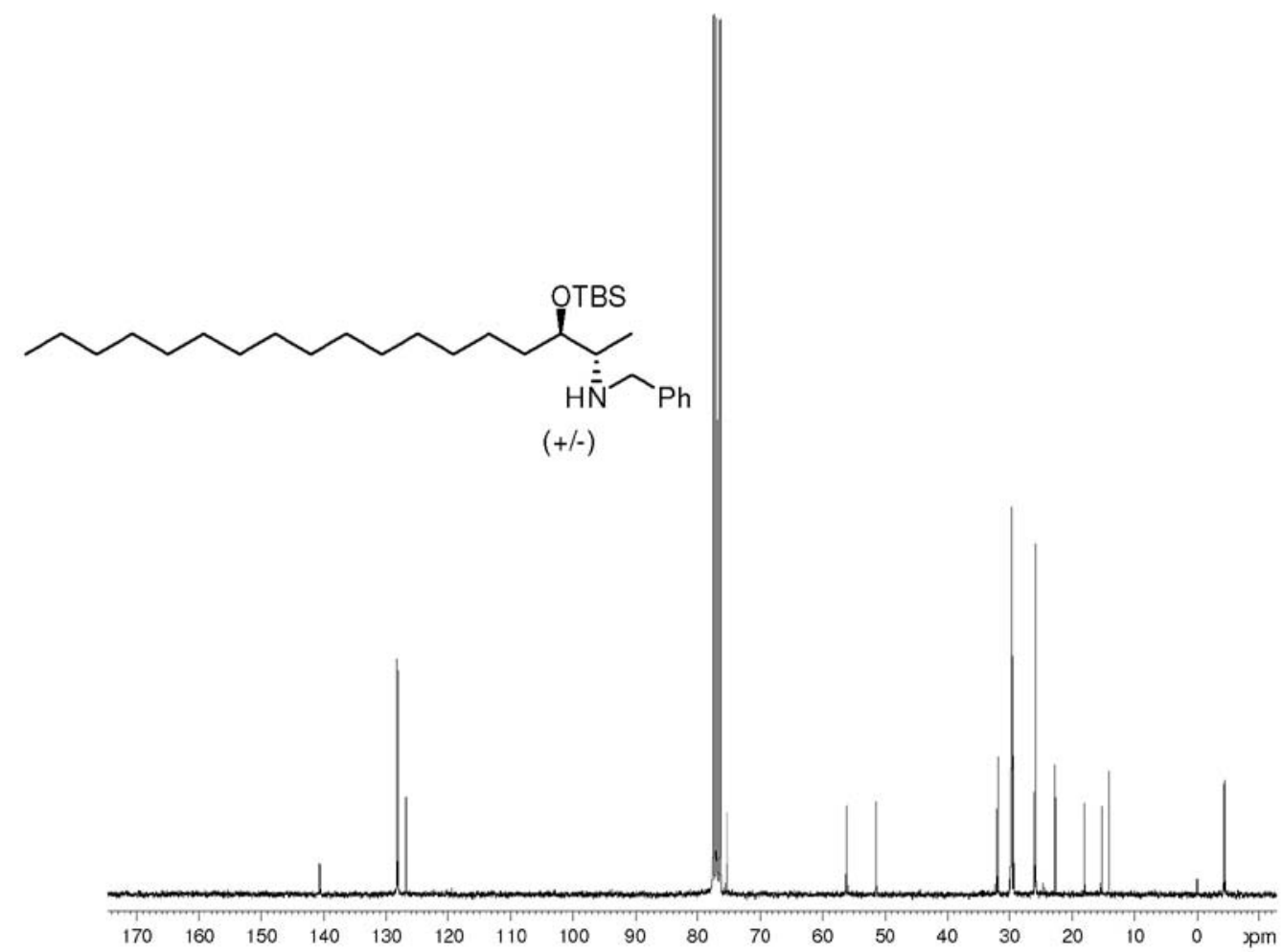

Figure S125. ${ }^{13} \mathrm{C}$ NMR $\left(\mathrm{CDCl}_{3}, 62.5 \mathrm{MHz}\right)$ of vicinal aminoalcohol 62. 


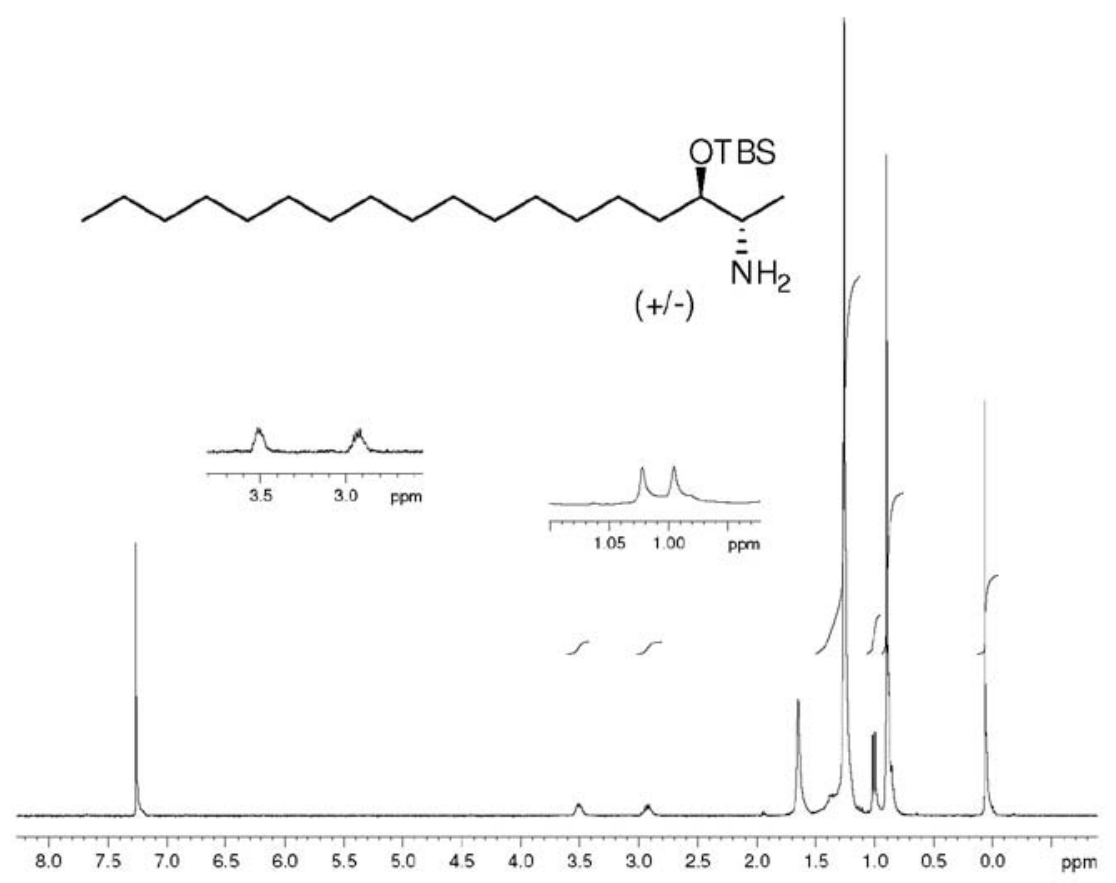

Figure S126. ${ }^{1} \mathrm{H} \mathrm{NMR}\left(\mathrm{CDCl}_{3}, 250 \mathrm{MHz}\right)$ of silylated aminoalcohol 63.

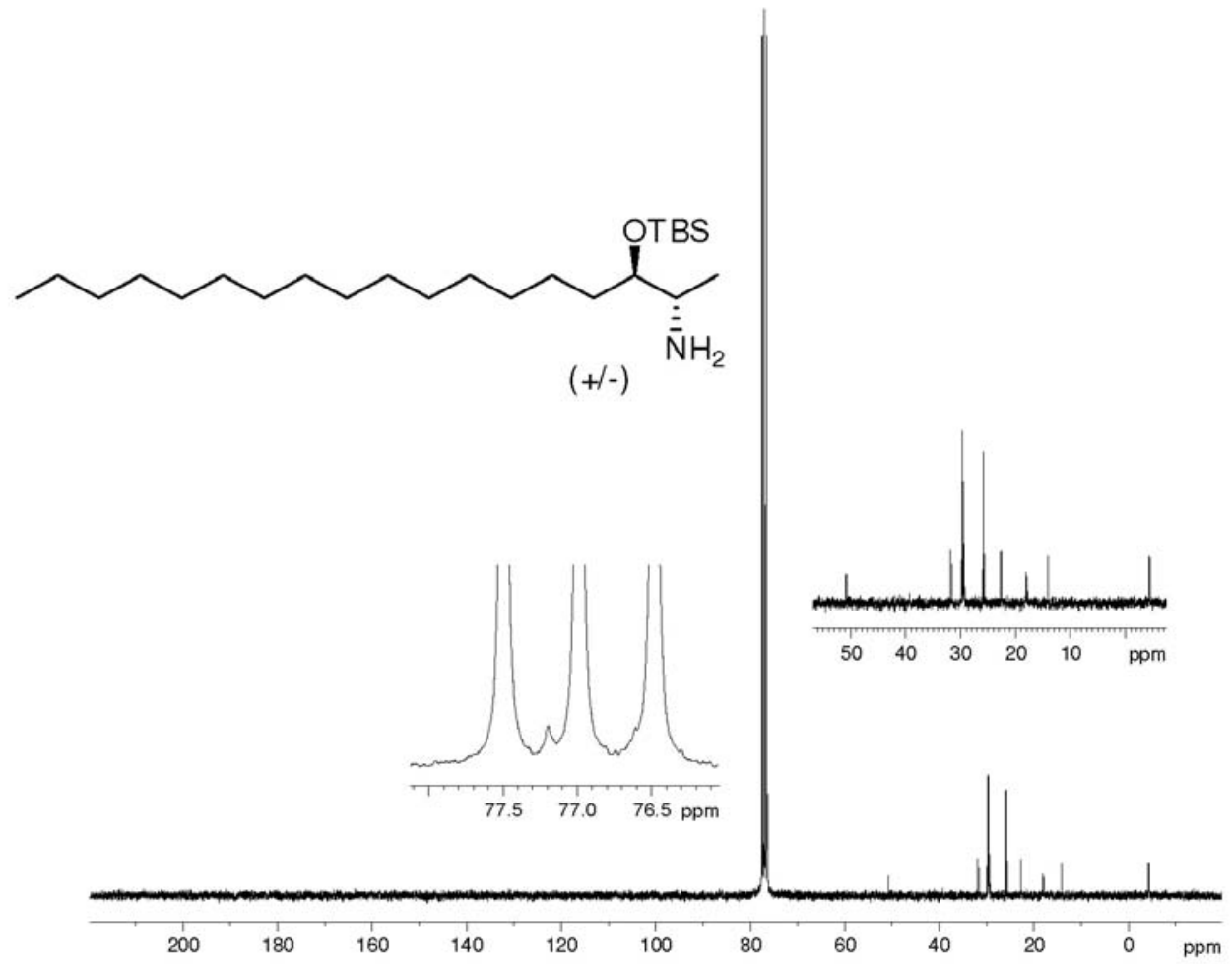

Figure S127. ${ }^{13} \mathrm{C}$ NMR $\left(\mathrm{CDCl}_{3}, 62.5 \mathrm{MHz}\right)$ of silylated aminoalcohol 63 . 


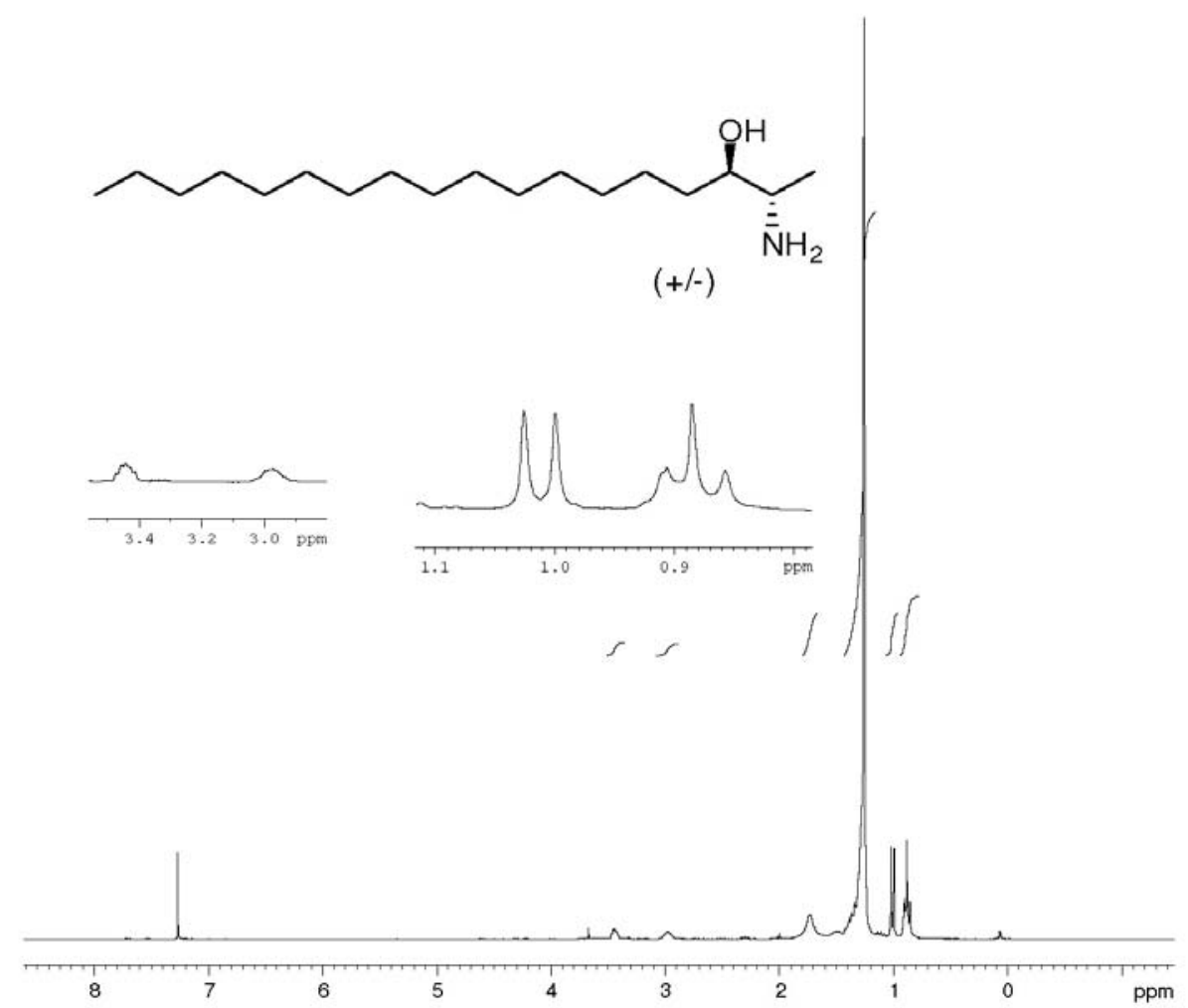

Figure S128. ${ }^{1} \mathrm{H}$ NMR $\left(\mathrm{CDCl}_{3}, 250 \mathrm{MHz}\right)$ of spisulosine (2).

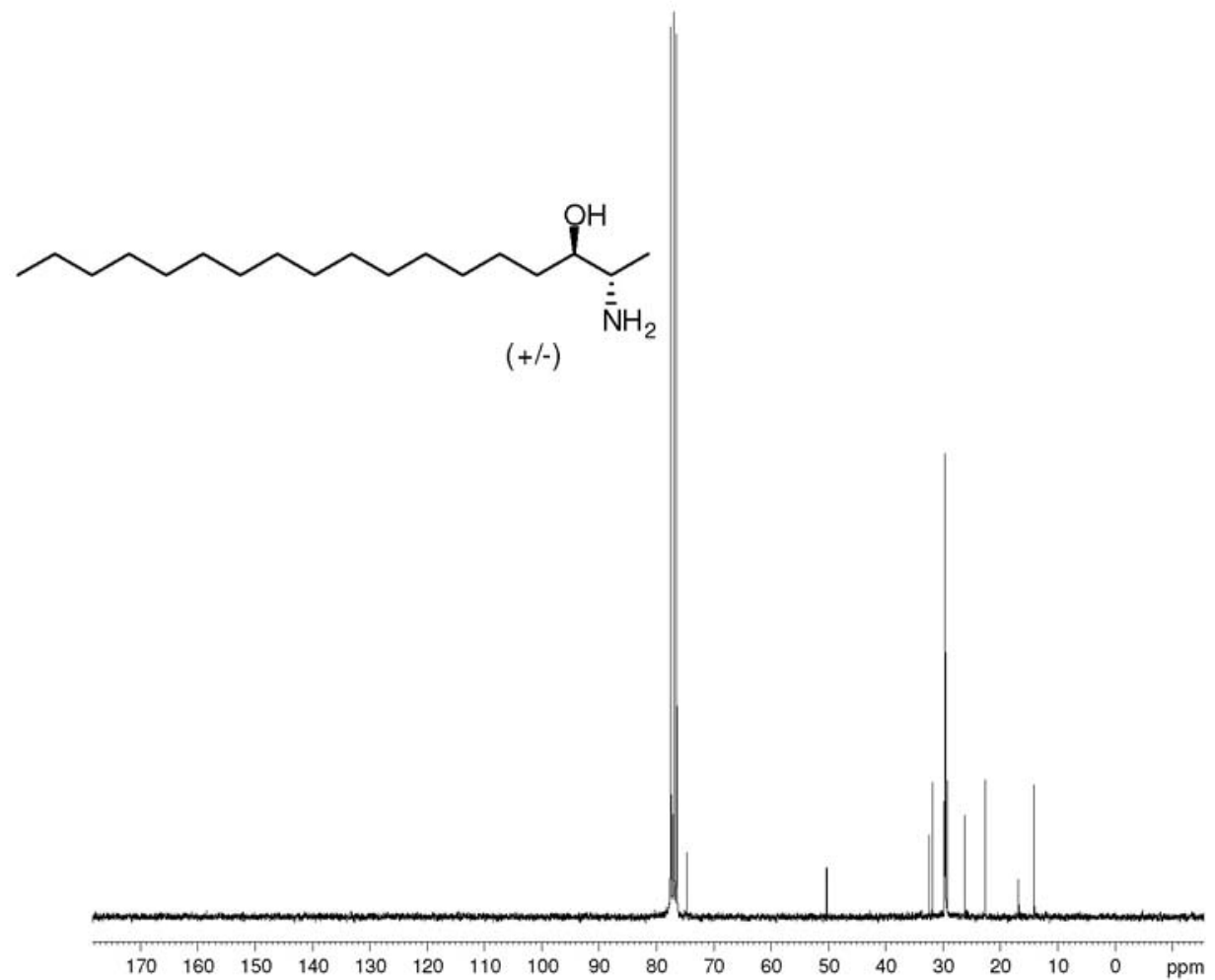

Figure S129. ${ }^{13} \mathrm{C} \mathrm{NMR}\left(\mathrm{CDCl}_{3}, 62.5 \mathrm{MHz}\right)$ of spisulosine (2). 